\title{
Experimentelle Untersuchungen des laminar-turbulenten Überganges der Zylindergrenzschichtströmung
}

\author{
Dissertation \\ zur Erlangung des Doktorgrades \\ der Mathematisch-Naturwissenschaftlichen Fakultäten \\ der Georg-August-Universität zu Göttingen
}

vorgelegt von

Burkhard Gölling

aus Rostock

Göttingen 2001 
D 7

Referent: Prof. Dr. rer. nat. Helmut Eckelmann

Korreferent: Priv. Doz. Dr. techn. Uwe Ch. Dallmann

Tag der mündlichen Prüfung: 3. 5. 2001 


\section{Inhaltsverzeichnis}

$\begin{array}{lr}\text { Liste der verwendeten Bezeichnungen } & \mathbf{v}\end{array}$

1 Einleitung 1

1.1 Einführung 1

1.2 Zum Stand der Forschung 5

$\begin{array}{lll}1.3 & \text { Vorgehensweise } & 11\end{array}$

2 Experimenteller Aufbau und Versuchsdurchführung 14

2.1 Hochdruckwindkanal Göttingen 14

2.2 Zylindermodelle $\quad 16$

2.2.1 „Referenz“"Zylindermodell 16

2.2.2 „Passiv“-Zylindermodell 17

2.2.3,Aktiv“-Zylindermodell 18

2.3 Messtechniken und Messverfahren $\quad 23$

2.3.1 Instationäre Kraftmessungen 23

2.3.2 Instationäre Wanddruckmessungen 24

2.3.3 Stationäre Wanddruckmessungen 24

2.3.4 Anstrichbilder 25

2.4 Datenerfassung $\quad 26$

2.4.1 Datenerfassung und Anlagensteuerung 26

\begin{tabular}{ll}
2.4 .2 & Instationäre Datenerfassung \\
\hline
\end{tabular}

2.5 Experimenteller Versuchsaufbau 28

2.6 Experimentelle Bestimmung physikalischer Größen 32

$3 \quad$ Ergebnisse zur natürlich abgelösten Zylinderströmung 35

3.1 Verhalten von Beiwerten und Strouhalzahl 35

3.2 Änderung des Ablöseverhaltens im unterkritischen Bereich 37

3.3 Kritischer Übergangsbereich $\quad 42$

3.4 Übergang vom überkritischen zum transkritischen Bereich 51

3.5 Der Zusammenhang zwischen Widerstands- und Basisdruckbeiwert 62

3.6 Aussagen für die Anwendung der Beeinflussungsverfahren 64 
4 Ergebnisse zum Verhalten der angeregten Zylindergrenzschicht $\quad 71$

4.1 Stationäre Störung mit spannweitig verteilten Wirbelerzeugern 71

4.1.1 Widerstands- und Auftriebsbeiwertverhalten 72

4.1.2 Asymmetrische Strömungszustände $\quad 74$

4.1.3 Spannweitige Steuerung spannweitig periodischer Ablösestrukturen $\quad 78$

4.2 Stationäre Störung der Grenzschicht durch Absaugen 80

4.2.1 Spannweitig homogene stationäre Störung durch Absaugen $\quad 80$

4.2.2 Spannweitig periodische stationäre Störung durch Absaugen 82

4.2.3 Einfluss der Variation der Absaugegeschwindigkeit 84

4.3 Instationäre Störung der Grenzschicht durch akustische Anregung 86

4.3.1 Spannweitig homogene Anwendung der akustischen Anregung 87

4.3.2 Spannweitig periodische Anwendung der akustischen Anregung 90

4.3.3 Zeitlich periodische Anwendung der akustischen Anregung 93

4.3.4 Einfluss der Variation der Schalldruckpegelamplitude $\quad 95$

5 Zusammenfassung $\quad 98$

$\begin{array}{ll}\text { Literaturverzeichnis } & 100\end{array}$

$\begin{array}{ll}\text { A Anhang } & \text { A-1 }\end{array}$

A.1 Re-Bereiche beim laminar-turbulenten Übergang der Zylindergrenzschicht A-1

A.2 Einteilung der Reynoldszahlbereiche nach verschiedenen Autoren A-2

A.3 Anstrichbilder der natürlich abgelösten Zylinderströmung A-5

A.4 Passiv gestörte Zylindergrenzschicht durch Wirbelerzeuger A-19

A.5 Re-gemittelte Widerstandsreduktionen bei aktiver Beeinflussung A-30

A.6 Re-Bereiche beim laminar-turbulenten Übergang der Zylindergrenzschicht A-32 


\section{Liste der verwendeten Bezeichnungen}

\section{Strömungsmechanische Größen und Bezeichnungen}

$b$

$b_{f}$

$\left|c_{A}\right| / \Delta f$

$\left|c_{P}\right| / \Delta f$

$c_{0}$

$c_{A, e f f}$

$c_{P, e f f}$

$d_{E}$

$d_{F}$

$d_{P}$

$f$

$f_{g}$

$f_{E}, f_{E, \max }$

$f_{H R}$

$f_{S}$

$k$

$l$

$l_{B}$

$n$

$p$

$p(i)$

$p \overparen{(f)}$

$p_{\infty}$

$p_{0}$

$p_{\text {eff }}$

$\Delta p_{D}$

$q_{\infty}$

$u^{\prime}, v^{\prime}, w^{\prime}$

$u^{+}$

$\left\langle u^{2}\right\rangle$

$\left\langle u^{\prime} w^{\prime}\right\rangle$

$\left\langle w^{2}\right\rangle$
Nachlaufbreite

lateraler Abstand der freien abgelösten Scherschichten im Ablösegebiet spektrale Amplitude des Auftriebsbeiwertes (Gleichung \{2.10\}) spektrale Amplitude der statischen Druckbeiwertes (Gleichung $\{2.14\}$ )

Schallgeschwindigkeit von Luft bei $20{ }^{\circ} \mathrm{C}$ und 1 bar, $c_{0}=343 \mathrm{~m} / \mathrm{s}$

Effektivwert der Schwankungen des Auftriebsbeiwertes (s.a. Gleichung $\{2.9\})$

Effektivwert der Schwankungen des statischen Druckbeiwertes (s. Gleichung $\{2.13\})$

Durchmesser der Bohrstege

Durchmesser der Fassungen für die Druckbohrungen

Durchmesser der Druckbohrungen

Frequenz bzw. Ablösefrequenz

Grenzfrequenz

akustische bzw. maximale akustische Anregungsfrequenz

Helmholtz-Resonator-Frequenz (Gleichung $\{2.1\}$ )

Sample-Frequenz

Kanalfaktor des Hochdruckwindkanals Göttingen ( $H D G)$

charakteristische Länge

Länge der Bohrstege zwischen Aktuatorkammern und Zylinderoberfläche

Anzahl der Bohrstege je Aktuatorkammer

statischer Wanddruck

Zeitreihe des statischen Druckes

Amplitudenspektrum des statischen Druckes

statischer Druck stromauf der Messstrecke (s.a. Bild 2.11)

Betriebsdruck des Hochdruckwindkanals (s.a. Bild 2.11)

Effektivwert der Schalldruckamplitude bei akustischer Anregung

statische Druckdifferenz zwischen Beruhigungskammer und Messstrecke sog. Düsendruckgefälle (s.a. Bild 2.11)

Staudruck der Anströmung

Komponenten des Vektors der Geschwindigkeitsschwankungen

Geschwindigkeit, definiert zu $u^{+}=\sqrt{\tau_{W} / \rho}$

Mittelwerte der Reynolds'schen Normalspannungen in Strömungsrichtung

Reynolds'sche Schubspannung

Reynolds'sche Normalspannung quer zur Strömungsrichtung 
$w_{Q}$

$w_{P}$

$x, y, z$

$x_{D}, y_{D}, z_{D}$

$y_{L}$

$\Delta y_{T G}$

$B$

$C_{p B}$

$C_{p B, E}$

$C_{g}$

$C_{A}$

$C_{P}$

$C_{W}$

$\Delta C_{W, r e l}$

$\overline{\Delta C_{W, r e l}}$

$D$

$F_{x 1}, F_{x 2}, F_{z}$

$F_{z}(i)$

$\widetilde{F_{z}(f)}$

G

$L$

$L_{f}$

$L_{p E}$

$L_{T}$

$L_{T G}$

$M$

$N$

$N_{S}$

$N_{S, O}$

$Q, Q_{0}$

$R_{g}$

$R_{Z}$

mittlere Absaugegeschwindigkeit in den Bohrstegen bei Absaugung durch $N$ Aktuatorkammern und bei gegebenem Volumenstrom $Q_{E}$ (s. Gleichung $\{2.2\})$

Schallschnelle bei akustischer Anregung (s. Gleichung $\{2.3\}$ )

Messstreckenkoordinaten

Zylinderkoordinaten, dimensionslos mit dem Zylinderdurchmesser $D$

spannweitige Länge einer Ablösestruktur bzw. Wirbelstruktur

spannweitiger Abstand von zwei benachbarten Wirbelerzeugern

Versperrungsverhältnis

statischer Basisdruckbeiwert, statischer Druckbeiwert im Heck des Zylinders bei einem Azimutwinkel von $180 \mathrm{Grad}$

statischer Basisdruckbeiwert einer passiv oder aktiv beeinflussten Zylinderströmung

Kapazität der Gegenkopplung im Ladungsverstärker

Auftriebsbeiwert (Gleichung $\{2.8\}$ )

statischer Druckbeiwert, definiert zu $C_{P}=\left(p-p_{\infty}\right) /\left(\frac{\rho}{2} U_{\infty}^{2}\right)$

Widerstandsbeiwert (Gleichung $\{2.7\}$ )

relative Widerstandsreduktion im Vergleich zur ungestörten, natürlich abgelösten Zylinderströmung

Reynoldszahl gemittelte Widerstandsreduktion im Vergleich zur ungestörten, natürlich abgelösten Zylinderströmung (Gleichung \{4.1\})

Außendurchmesser des Zylindermodells

Kraftkomponenten, gemessen mit der Piezowaage

Zeitreihe der Auftriebskraft

Amplitudenspektrum der Auftriebskraft

Kompressionsmodul von Luft

Länge des Zylinders

Rückkopplungslänge (Gleichung \{3.3\})

Schalldruckpegel bei akustischer Anregung in $\mathrm{dB}$ mit $p_{\text {eff }, 0}=2,0^{\times} 10^{-5} \mathrm{~Pa}$

Länge einer Trennplatte im Heck des Zylinders

normale Höhe eines Wirbelerzeugers

Anzahl über die zu mittelnden Spektren bei der Fourieranalyse von Zeitreihen aus Kraft- und Druckmessungen

Anzahl der Kammern des Aktuatormoduls

Anzahl der Sample einer Zeitreihe bei der Fouriertransformation

Anzahl der Sample einer Zeitreihe bei Kraft- und Druckmessungen

Volumenstrom beim Absaugen und Messbereich eines Durchflussmessers

Widerstand der Gegenkopplung im Ladungsverstärker

räumlich gemittelte Rauigkeitshöhe 
$R e$

$R e_{D}$

$R e_{k r i t, A}$

$\operatorname{Re}_{\text {krit }, A^{*}}$

$R e_{\text {krit }, B}$

$\operatorname{Re}_{k r i t, B^{*}}$

$\operatorname{Re}_{\text {krit }, C}$

$\operatorname{Re}_{k r i t, D}$

$S, S_{b}, S_{t}$

$S_{V}$

$\mathrm{Sr}$

$S r_{f}$

$\mathrm{Sr}_{E}$

$S r_{L f / \lambda_{Q W}}$

$S r_{R}$

$T_{0}$

$T_{g}$

$U, V, W$

$U_{\infty}, U_{\infty, \text { max }}$

$U_{c}$

$V_{K}$

$W$

$W P$
Reynoldszahl mit $\operatorname{Re}=U l / v$ (s.a. Gleichung $\{1.2\}$ )

Reynoldszahl bezogen auf den Durchmesser des Zylindermodells (s.a. Gleichung $\{2.6\}$ )

kritische Reynoldszahl für „Transition A“

kritische Reynoldszahl für „Transition $A$ *“

kritische Reynoldszahl für „Transition $B^{\text {“ }}$

kritische Reynoldszahl für „Transition $B$ *“

kritische Reynoldszahl für „Transition $C^{\circ}$

kritische Reynoldszahl für „Transition $D^{\text {“ }}$

Ort der zeitlich gemittelten Ablösung: allgemein, laminar, turbulent

Seitenverhältnis des Zylinders, $S_{V}=L / D$

Strouhalzahl (Gleichung \{1.1\}) bzw. Strouhalzahl der Wirbelablösefrequenz ermittelt aus den Auftriebs- bzw. Druckschwankungen (Gleichung $\{2.11\}$ )

Strouhalzahl bezogen auf die Rückkopplungslänge $L_{f}$, definiert zu $S r_{f}=L_{f} f / U_{\infty}$ (s.a. Gleichung $\{3.4\}$ )

Strouhalzahl der akustischen Anregungsfrequenz mit $S r_{E}=D f_{E} / U_{\infty}$

Strouhalzahl bezogen auf die Rückkopplungslänge und die Wellenlänge der Querwirbel im Nachlauf (Gleichung \{3.6\})

universelle Strouhalzahl nach Roshko (1954) [70], gegeben zu $S t_{R}=f b_{f} / \sqrt{1-C_{p B}} U_{\infty}$

Temperatur der Luft im Windkanal

Zeitkonstante des Ladungsverstärkers

x-, y- und z-Komponente der Geschwindigkeit im modellfesten Koordinatensystem

Anströmgeschwindigkeit und maximale Anströmgeschwindigkeit (s.a. Gleichung $\{2.5\}$ )

Konvektivgeschwindigkeit der Querwirbel im Nachlauf

Volumen einer Kammer des Aktuatomoduls, gegeben $\mathrm{zu} V_{K}=12 \times 4 \times 5 \mathrm{~mm}^{3}$

Widerstandskraft (s.a. Gleichung $\{1.3\}$ )

Wendepunkt im Geschwindigkeitsprofil (z.B. im Bild 1.2)

\section{Griechische Symbole}

$\lambda$

$\mu$

$v$

$\rho$

$\rho_{0}$

$\tau_{W}$

$\varphi$
Wellenlänge

dynamische Zähigkeit

kinematische Zähigkeit

Dichte eines Fluids

Dichte des strömenden Fluids im Windkanal (s.a. Gleichung $\{2.4\}$ )

Wandschubspannung

Azimutwinkel 


$\begin{array}{ll}\varphi_{p} & \text { Azimutwinkel der Druckbohrungen } \\ \varphi_{D r} & \text { azimutaler Drehwinkel } \\ \varphi_{E} & \text { Azimutwinkel der akustischen Anregung } \\ \varphi_{S} & \text { Azimutwinkel der Ablösung } \\ \varphi_{S, \text { oben }} & \text { Azimutwinkel der Ablösung auf der Oberseite des Zylinders } \\ \varphi_{S, \text { unten }} & \text { Azimutwinkel der Ablösung auf der Unterseite des Zylinders } \\ \varphi_{T G} & \text { Azimutposition der Wirbelerzeuger beim „Passiv“-Zylindermodell } \\ \Delta \varphi_{D r} & \text { azimutaler Drehwinkelbereich } \\ \Delta \varphi_{S} & \text { Differenz der Azimutwinkel der Ablöseorte über den hinteren Staupunkt } \\ \Psi_{S} & \text { Stromlinie der zeitlich gemittelten Ablösung }\end{array}$

\section{Sonstige Bezeichnungen}

\begin{tabular}{|c|c|}
\hline $1 M G$ & 1-Meter-Windkanal Göttingen \\
\hline$B G$ & Bloor-Gerrard \\
\hline$D e A s$ & Datenerfassung und Anlagensteuerung \\
\hline$D L R$ & Deutsches Zentrum für Luft- und Laumfahrt e.V. \\
\hline$H D G$ & Hochdruckwindkanal Göttingen \\
\hline$I D a$ & Instationäre Datenerfassung \\
\hline KH & Kelvin-Helmholtz \\
\hline$K W$ & von-Kàrmàn-Wirbel \\
\hline$S L$ & Schiller-Linke \\
\hline $\operatorname{Tr} A$ & „Transition A“ bei zunehmender Reynoldszahl im kritischen Bereich \\
\hline $\operatorname{Tr} A^{*}$ & „Transition $A^{* “}$ bei abnehmender Reynoldszahl im kritischen Bereich \\
\hline $\operatorname{Tr} B$ & „Transition $B_{“}$ “ bei zunehmender Reynoldszahl im kritischen Bereich \\
\hline $\operatorname{Tr} B^{*}$ & „Transition $B *$ “ bei abnehmender Reynoldszahl im kritischen Bereich \\
\hline $\operatorname{Tr} C$ & „Transition $C$ “ bei zunehmender Reynoldszahl im oberen Übergangsbereich \\
\hline $\operatorname{Tr} D$ & „Transition $D_{“}$ mit zunehmender Reynoldszahl im oberen Übergangsbereich \\
\hline$T S$ & Tollmien-Schlichting \\
\hline
\end{tabular}




\section{Einleitung}

Das Verhalten einer sich zeitlich und örtlich verändernden Strömung kann durch die Bestimmung von zeitlich gemittelten Größen und von zeitabhängigen Schwankungsgrößen an jedem Ort mit viel Aufwand erfasst werden. Das Grundlegende einer Strömung ist aber in ihrem Ordnungszustand und in ihrer Dynamik zu sehen. Daher muss mit den gemessenen oder gerechneten Strömungsfelddaten eine Modellierung der Strömungsstruktur und der Strömungsvorgänge möglich sein. Mit der Modellierung wird eine vereinfachende, aber wesentliche Beschreibung eines Strömungsverhaltens in Abhängigkeit von charakteristischen Parametern sowie Anfangs- bzw. Randbedingungen für bestimmte Strömungsklassen erhalten. Ist das strukturelle und dynamische Strömungsverhalten verstanden, können besonders effiziente Steuerungsverfahren für unterschiedliche Ziele entwickelt und eingesetzt werden.

\subsection{Einführung}

Eine wichtige, in der Praxis häufig anzutreffende Klasse von Strömungen ist die der abgelösten Strömungen. Abgelöste Strömungen treten z.B. in Strömungsmaschinen, bei Kraftfahrzeugen und Tragflügeln auf. Dabei wird von einer abgelösten Strömung gesprochen, wenn die zunächst am Körper anliegende Strömung mindestens an einer Stelle der Wand sich vom Körper wegbewegt, also der Körperkontur nicht mehr folgt. Mit der Ablösung verbunden ist die Ausbildung von unterschiedlichen Wirbelstrukturen, die das Widerstandsverhalten des Körpers maßgeblich beeinflussen. Das Verstehen dieser Strömungsvorgänge und die Möglichkeit ihrer Beeinflussung, z.B. zur Widerstandsreduktion, stellt somit einen wichtigen wirtschaftlichen Faktor dar. Für die experimentelle Untersuchung abgelöster Strömungen bietet sich aufgrund seiner einfachen Geometrie der querangeströmte Zylinder mit kreisförmigen Querschnitt an. Obwohl diese Strömung nominell zweidimensional ist, werden in Abhängigkeit vom Strömungszustand unterschiedliche räumlich zeitliche Ablösestrukturen beobachtet. Dieses Strömungsverhalten zu verstehen ist Gegenstand der vorliegenden Arbeit.

Ein markantes Phänomen bei der Strömungsablösung am Zylinder untersuchte Strouhal 1878 [85]. Er bemerkte, dass bei der Anströmung von Kreiszylindern ein Ton zu hören war. Der hörbare Ton entsteht durch Wirbel, die sich stromab des Zylinders periodisch ausbilden. Er stellte fest, dass die Wirbelablösefrequenz $f$ sich proportional zur Anströmgeschwindigkeit $U$ und umgekehrt proportional zum Durchmesser $D$ verhält. Hieraus ergibt sich die nach ihm benannte charakteristische Kennzahl - die Strouhalzahl Sr zur Beschreibung periodischer Wirbelablösung mit

$$
S r=\frac{f D}{U} .
$$

Aus unserem Erfahrungsbereich ist weiterhin bekannt, dass ein bewegter Körper in einer realen, d.h. reibungsbehafteten Strömung einen Widerstand erfährt. Der Gesamtwiderstand $C_{W}$ setzt sich dabei aus dem Reibungswiderstand und dem Druckwiderstand zusammen. Soll der Körper seine Bewegung mit der Geschwindigkeit $U$ kontinuierlich fortsetzen, muss kinetische Energie aufgewendet werden. Aus dem Verhältnis von Trägheitskraft zur Reibungskraft beschrieb Rey- 
nolds 1883 [66] eine weitere charakteristische Kennzahl - die Reynoldszahl Re mit

$$
R e=\frac{\rho l U}{\mu},
$$

wobei $l$ eine charakteristische Länge des Körpers beschreibt sowie $\rho$ die Dichte und $\mu$ die dynamische Viskosität des Fluids darstellt. Ist die Reynoldszahl klein, überwiegen bei anliegender Strömung die Reibungskräfte, also der Reibungswiderstand. Er wird mit zunehmender Reynoldszahl bei gleichem Ordnungszustand der Strömung kleiner. Tritt allerdings Ablösung auf, so überwiegen die Druckkräfte und der Druckwiderstand ist maßgeblich. So erkannte bereits Reynolds, dass der Widerstand für eine gegebene Körperform eine Funktion der Reynoldszahl sein muss.

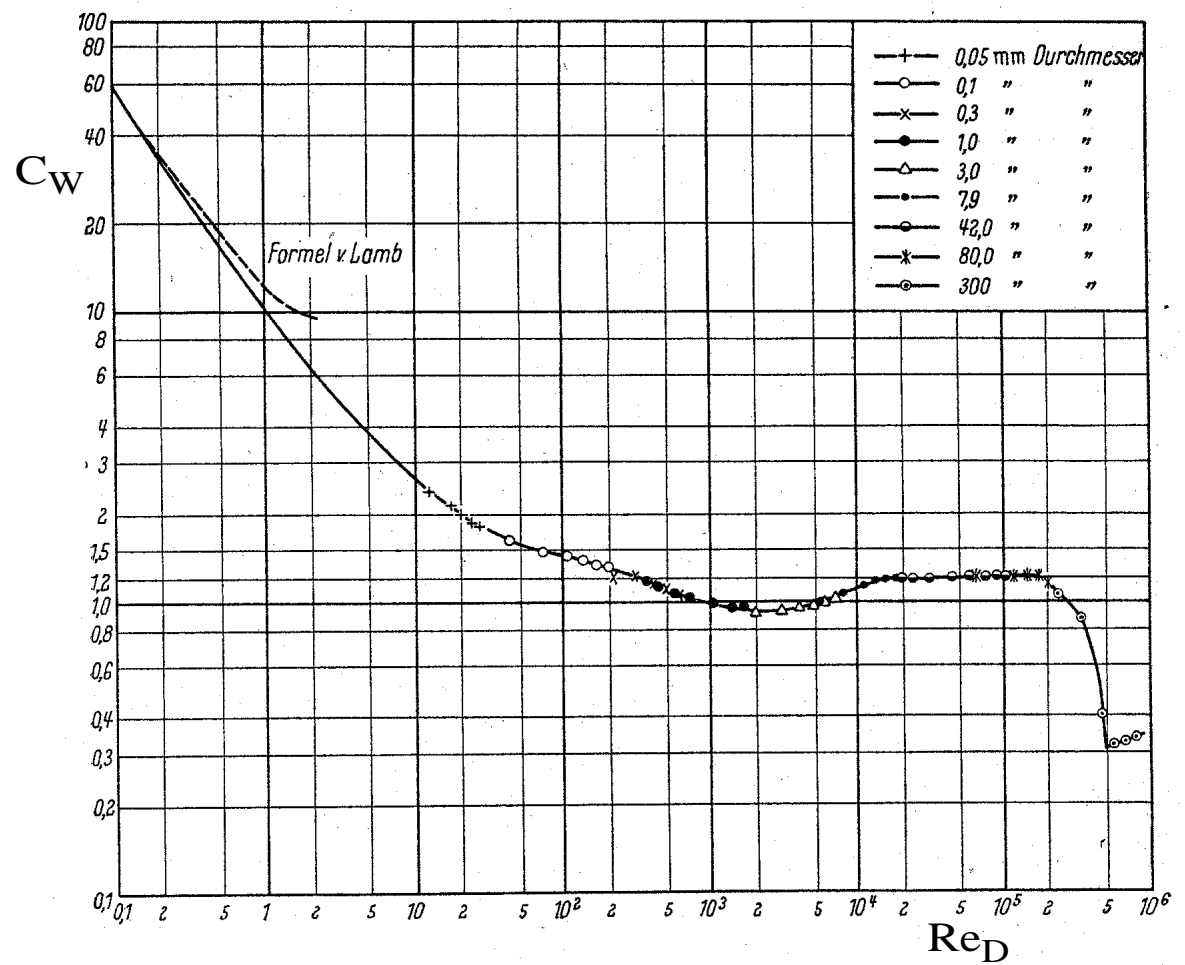

Bild 1.1 Widerstandsbeiwert in Abhängigkeit von der Reynoldszahl nach Messungen von Wieselsberger (aus Prandtl 1921) [61].

Das Phänomen der Ablösung war damit aber nicht verstanden. Erst Ludwig Prandtl fand Anfang des 20. Jahrhunderts die Ursache für das Widerstandsverhalten von sog. stumpfen Körpern. Prandtl (1904) [59] konnte durch experimentelle Untersuchungen z.B. an der ebenen Plattenströmung und an der Zylinderströmung in einem „Versuchsapparat“, einem Wasserkanal, zeigen, dass eine Grenzschicht zwischen der Wand des Körpers und der freien Außenströmung existiert: „In der schmalen Übergangsschicht ergeben dann die schroffen Geschwindigkeitsunterschiede trotz der kleinen Reibungskonstanten merkliche Wirkungen." Für das Auftreten von Ablösungen, schloss Prandtl weiter, ,ist die notwendige Bedingung für das Ablösen des Strahles die, daß längs der Wand in der Richtung der Strömung eine Drucksteigerung vorhanden ist". Weiterhin beobachtete Prandtl in dem „Versuchsapparat“ je nach Umlaufgeschwindigkeit des Wasserrades die periodische Ausbildung von Wirbeln hinter einem kreisförmigen Zylinder. Daraufhin untersuchte von Kármán (1911) [93] die Stabilität der Wirbelschicht auf einfache sta- 
bile Anordnungen von sog. Wirbelfäden. Wirbelfäden stellen den Grenzfall zur Beschreibung der Wirbelstärke einer sich mit der Stromabentwicklung aufwickelnden Wirbelschicht dar. So fand er, dass nur die spezielle Anordnung der Wirbelfäden mit entgegengesetztem Vorzeichen der Wirbelstärke in einem gewissen Abstand voneinander stabil ist. Weiterhin entsteht in dem hinterem Bereich des Zylinders ein Gebiet des Unterdruckes, der ,bei vielen Körperformen geradezu maßgebend für die Größe des Widerstandes erscheint" (von Kármán 1911 [93]). Mit weiteren systematischen Untersuchungen an der Kreiszylinderströmung durch Wieselsberger (1923) [95] wurde schließlich der formelle Zusammenhang zwischen Widerstandskraft $W$ und Reynoldszahl gefunden:

$$
W=C_{W}(R e) F \frac{\rho}{2} U^{2},
$$

wobei $F$ die Querschnittsfläche des Körpers beschreibt. „Das Ähnlichkeitsgesetz kommt dadurch zum Ausdruck, daß bei der gewählten Auftragung nach Reynoldsschen Zahlen sich die Ergebnisse sämtlicher Versuchsreihen zwanglos zu einer einzigen Kurve zusammenschließen" (Prandtl 1921 [61], vgl. auch Prandtl 1914 [60]), wie in Bild 1.1 zu sehen. Mikroskopisch beruhen sowohl die Ausbildung der Grenzschicht als auch das Phänomen der Ablösung auf der Möglichkeit der Fluidteilchen in einer inkompressiblen realen Strömung mit kleinem Reibungskoeffizienten sowohl in als auch quer zur Strömungsrichtung Impuls auszutauschen. Makroskopisch führt dies zur Ausbildung einer Scherschichtströmung mit Schichten unterschiedlicher Geschwindigkeit. An der Wand ist die Geschwindigkeit aufgrund der Haftbedingung Null und bei ausreichend großem Abstand von der Wand, knapp oberhalb der Grenzschichtdicke, nimmt sie die der äußeren Strömung an. Das Geschwindigkeitsprofil kann demnach in Abhängigkeit von dem aufgeprägten Druckgradienten unterschiedliche Formen aufweisen (siehe Bild 1.2). Wirkt in Strömungsrichtung ein positiver Druckgradient, $d p / d x>0$, so nimmt die kinetische Energie der Grenzschichtströmung ab, während die potentielle Energie gleichermaßen ansteigt. Die Schubspannung an der Wand, $\tau_{W}=\mu \cdot \partial u / \partial y$, nimmt ebenfalls ab und zwischen der Wand und der Außenströmung bildet sich im Geschwindigkeitsprofil ein Wendepunkt WP aus, d.h. es gilt für einen bestimmten wandnormalen Abstand $\partial^{2} u / \partial y^{2}=0$. Aufgrund des positiven Druckgradienten wird also eine Verzögerung der Grenzschichtströmung und schließlich eine Umkehr der Strömungsrichtung in der Nähe der Wand verursacht und die Wandschubspannung ändert ihr Vorzeichen. Für die Ablösung am Ort $S$ muss also neben die notwendigen Bedingung, des Auftretens eines Wendepunktes im Geschwindigkeitsprofil, noch die hinreichende Bedingung, des Verschwindens der Wandschubspannung, $\tau_{W}=0$, erfüllt sein (Prandtl 1926 [63]). Für Positionen stromab der Ablösestelle bildet sich aufgrund der Rückströmung an der Wand phänomenologisch eine ablösende Grenzschicht aus.

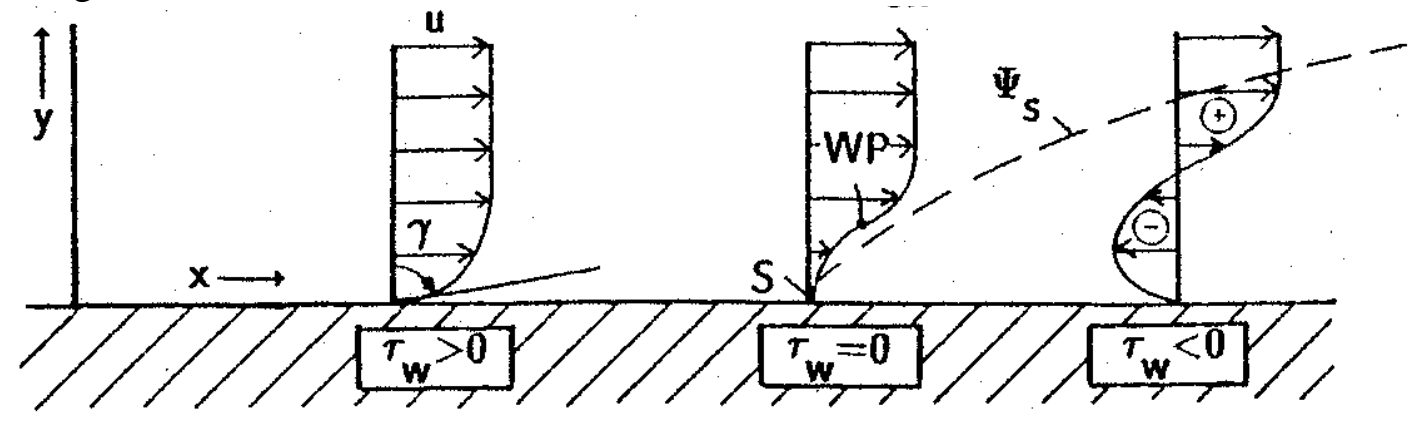

Bild 1.2 Schematische Darstellung der Ablösung für eine zweidimensionale Grenzschichtströmung (aus Leder 1992 [43]; Erklärung des Bildes siehe Text) 
Eine weitere Art der Ablösung resultiert aus den geometrischen Randbedingungen des Körpers. Obwohl eine Strömung beschleunigt ist und auch die Wandschubspannung ungleich Null beträgt kommt es zur Ablösung bzw. zum sog. Strömungsabriss. Die Strömung kann dann trotz negativem Druckgradienten in Strömungsrichtung der Körperkontur nicht mehr folgen. Sie tritt z.B. bei Verzweigungen, Krümmern und unstetigen Querschnittsveränderungen in Rohren und Kanälen sowie bei Körpern auf, die „,scharfe Kanten“ besitzen. Auch die Hinterkantenablösung z.B. bei einer nichtangestellten Platte oder Profil ist dieser Art der Strömungsablösung zuzuzählen.

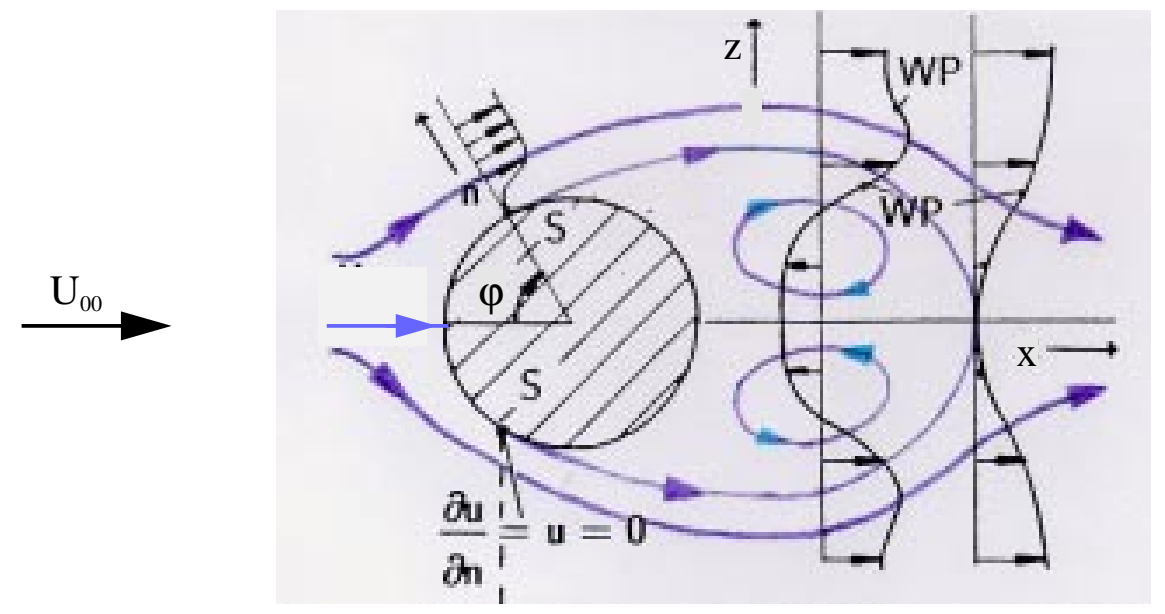

Bild 1.3 Schematische Darstellung der abgelösten zweidimensionalen Zylinderströmung (aus Leder 1992 [43]). Aufgrund der Beschleunigung der Strömung über den Schultern des Zylinders entstehen auch im Geschwindigkeitsprofil der Scherschichten und des Nachlaufs Wendepunkte. Die zeitlich gemittelte Stromlinie mit der Geschwindigkeit Null umfasst dabei das Rezirkulationsgebiet und die freien abgelösten Scherschichten auf jeder Seite des Zylinders. (Bem.: $n$ bezeichnet hier die Normalenrichtung der Zylinderoberfläche.)

Wenden wir die phänomenologische Betrachtungsweise der zweidimensionalen Ablösung auf eine Kreiszylinderumströmung an, so ergibt sich das Bild 1.3. Die zeitlich gemittelte Stromlinie mit der Geschwindigkeit Null beschreibt das Ablösegebiet im Heck des Zylinders und umfasst sowohl das Rückstromgebiet als auch die zwischen ihr und der Außenströmung entstehende freie abgelöste Scherschicht (Leder 1992 [43]). Stromab des Rückstromgebietes befindet sich der Nachlaufbereich. In ihm verläuft die Strömung im zeitlichen Mittel in Richtung der Außenströmung, wobei durch die instationäre Wechselwirkung der abgelösten freien Scherschichten periodische Schwankungen die Nachlaufströmung überlagern. In Abhängigkeit von der Reynoldszahl kommt es zur Ausbildung von Querwirbeln, den sog. von-Kármán-Wirbeln, die mit der Nachlaufströmung konvektiv abschwimmen.

Diese Querwirbelstruktur ist für große Reynoldszahlbereiche stabil. Die damit verbundene topologische Struktur der Ablösung am Zylinder könnte mit einem zweidimensionalen Eigenwertproblem der Jakobimatrix z.B. des zweidimensionalen Geschwindigkeitsvektorfeldes beschrieben werden. Die Anzahl der möglichen singulären Lösungen ist endlich und hängt von den Werten der Eigenwerte ab. Sie liefern elementare topologische Strukturen, wie Knoten, Sattel oder Fixpunkt, und geben das Instabilitätsverhalten der entsprechenden Strukturen an (Blaquière 1966 [10], S. 108ff). 

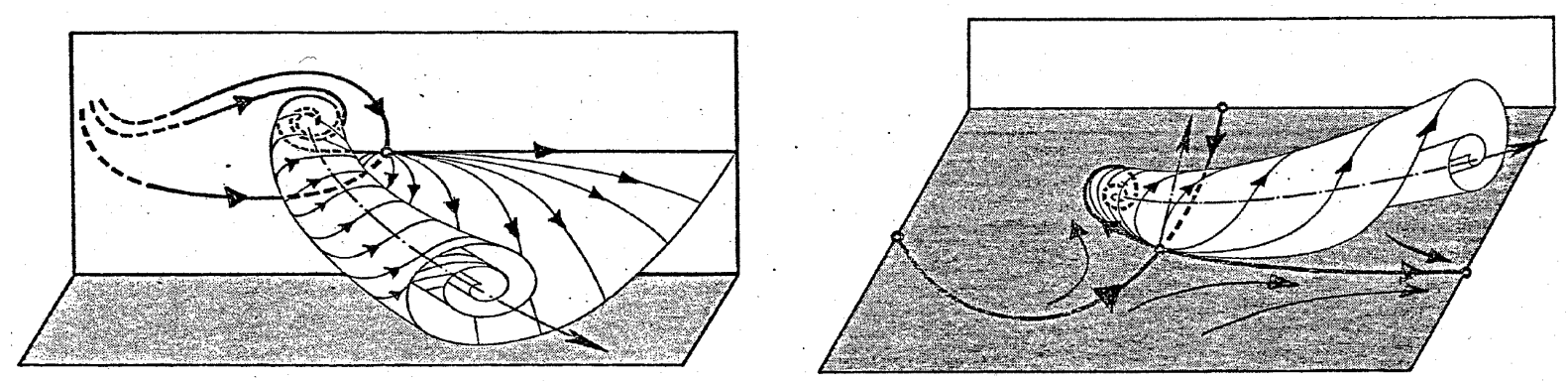

Bild 1.4 Transition zwischen einer u-förmigen Querwirbelstruktur (links) und einer Längswirbelstruktur (rechts), die von der Zylinderoberfläche ablöst. Alle Stromlinien, welche die singuläre Stromfläche bilden, stammen aus dem Sattelpunkt der Ablösung und wickeln die einzelne Stromlinie, welche den Fokus verlässt, ein (aus Dallmann 1983 [19]).

Das zweidimensionale Problem wird in drei Dimensionen zunehmend komplex und verschiedene Klassen von Singularitäten liefern unterschiedliche dreidimensional ablösende Wirbelstrukturen. Beispiele topologischer dreidimensionaler Strukturen sind eine u-förmige Querwirbelstruktur und eine Längswirbelstruktur (Bild 1.4). Beide Strukturen werden durch die topologische Struktur eines Sattels beschrieben, wobei alle Stromlinien, die vom Sattelpunkt kommen, die singuläre Stromfläche bilden und die einzelne Stromlinie, die den Fokus verlässt, einwickeln (Dallmann 1983 [19], Dallman et al. 1995 [21]). Beide topologische Strukturen sind ineinander durch bijektive Abbildungen überführbar, so dass durch die Wirkung von Instabilitäten Übergänge von der einen zu der anderen Struktur möglich sind.

Mit der vorliegenden Arbeit zur experimentellen Untersuchung des laminar-turbulenten Überganges der Zylindergrenzschichtströmung werden daher drei Ziele verfolgt. Als Erstes sollen die Übergänge, wo die Querwirbelablösung instabil wird, bestimmt werden. Zum Zweiten gilt es, die bei diesen Übergängen auftretenden Wirbelstrukturen zu identifizieren und ihre räumlichen und zeitlichen Skalen anzugeben. Als Drittes, sollen künstlich derartige Wirbelstrukturen insbesondere in Reynoldszahlbereichen mit großem Widerstandsbeiwert angeregt werden, um zum einen das Instabilitätsverhalten der ablösenden Zylindergrenzschicht zu beobachten und zum anderen widerstandsreduzierende Effekte zu erzielen. Um gezielt vorzugehen, wird daher zunächst auf den Erkenntnisstand der Zylinderforschung eingegangen.

\subsection{Zum Stand der Forschung}

Das Verhalten der Zylinderströmung und die Entstehung von Wirbelstrukturen beruht auf ihrem Instabilitätsverhalten. Die Grundströmung und die mit ihr entstehenden Scherschichten sind für bestimmte Reynoldszahlen instabil, so dass ein komplexes Szenario von aufeinanderfolgenden Instabilitäten die Ausbildung von Wirbelstrukturen im Nachlauf, in den freien abgelösten Scherschichten und in der Grenzschicht verursachen. Dabei werden jeweils drei unterschiedliche Ordnungszustände der Scherschichten unterschieden: laminar, transitionell und turbulent. Im laminaren Zustand liegen die Schichten unterschiedlicher Geschwindigkeiten geordnet übereinander. Im transitionellen Zustand beginnt zunehmend eine Vermischung von Fluidteilchen kleinerer und größerer Geschwindigkeiten in einer Schicht und die geordnete Schicht- 
struktur ist nur noch teilweise gegeben. Im turbulenten Zustand erreicht die Vermischung ihren Höhepunkt und ungeordnete kleinskalige Wirbel prägen die Struktur der Scherschicht.

Bei sehr kleinen Reynoldszahlen ist zunächst die vollständige laminare Umströmung des Zylinders zu beobachten. Aber bereits ab einer Reynoldszahl von $R e_{D}=5$ sind auch die Reibungskräfte der schleichenden Strömung nicht groß genug, um eine stationäre Ablösung zu verhindern. Mit zunehmender Reynoldszahl erweist sich die Grundströmung als global instabil, so dass es bei einer Reynoldszahl von $R e_{D}=47$ zur periodisch wechselseitigen Ablösung der Grenzschicht vom Zylinder kommt (Koch 1985 [39]). Mit dieser periodischen Ablösung entstehen im Rezirkulationsgebiet stabile Querwirbel, die spannweitig homogen vom Zylinder abschwimmen. Diese Querwirbel sind die sog. von-Kármán-Wirbel, die im Nachlauf die sog. Kármán'sche Wirbelstraße bilden. Diese Querwirbelstruktur ist eine den Nachlauf bestimmende Wirbelstruktur und wird bis zu großen Reynoldszahlen von $R e_{D} \approx 10^{9}$, z.B. bei der Umströmung der Insel „Jan Mayen“ beobachtet. Lediglich wenn der Nachlauf oder die o.g. Scherschichten instabil werden und ein transitioneller Strömungszustand vorliegt, werden diese Querwirbel durch eine andere Wirbelstruktur, von sich in Strömungrichtung ausbildenden Wirbeln, sog. Längswirbel, beeinflusst oder unterdrückt. Darüberhinaus kann es auch zur Schrägablösung der Querwirbel kommen. So treten mit Beginn der Parallelablösung der von-KármánWirbel in dem Reynoldszahlbereich von $R e_{D}=48$ bis $R e_{D}=160$ auch mehrere Moden der Schrägablösung auf. Diese sind nicht auf unsauber definierte Randbedingungen zurückzuführen, sondern werden natürlicherweise beobachtet (König 1993 [41]). Diese stabilen Anordnungen der von-Kármán-Wirbelstraße, auch Moden genannt (König et al. 1993 [42]), wird aber auch instabil gegenüber spannweitigen Moden. Mit der Schrägablösung treten auch Wirbelverzweigungen auf, die andererseits durch definierte Randbedingungen unterdrückt werden können (Eisenlohr und Eckelmann 1988 [25]). Bei der Transition des Nachlaufes treten drei grundsätzlich verschiedene Moden in Abhängigkeit von der Reynoldszahl auf. Mit der Reynoldszahl von $R e_{D}=160$ geht der Nachlauf in einen anderen Zustand über. Die Querwirbelröhren bleiben spannweitig lokal am Zylinder „haften“, so dass bananenförmig gekrümmte Querwirbelröhren entstehen (Zhang et al. 1995 [102]). Eine charakteristische spannweitige Wellenlänge bildet dabei diese sog. Wirbelhaftmode nicht aus. Sie ist dafür verantwortlich, dass sich mit der weiteren Stromabentwicklung der Nachlauf spannweitig deformiert. Im Reynoldszahlbereich von $R e_{D}=180$ bis $R e_{D}=240$ wird die sog. A-Mode im Nachlauf beobachtet, die sich durch eine große spannweitige Wellenlänge von etwa $y_{L}=4 D$ auszeichnet (Brede et al. 1996 [15], Williamson 1996 [96]). Die Ausbildung dieser Mode kann auf eine Zentrifugalinstabilität der abgelösten Scherschicht oder der Primärwirbel zurückgeführt werden (Zhang et al. 1994 [101]), die dann mit der weiteren Stromabentwicklung verstärkt wird. Unter einem Primärwirbel wird dabei der Wirbel verstanden, der während einer halben Periode der Ablösung im Rezirkulationsgebiet entsteht und dann abschwimmt, und stromab den von-Kármán-Wirbel bildet. Das Auftreten der A-Mode beinflusst die zur Zylinderachse parallele Ablösung der Querwirbel sehr stark, so dass es zu einer spannweitigen Deformation der Wirbelröhren kommt. Anders verhält sich die B-Mode. Sie wird ab einer Reynoldszahl von etwa $\operatorname{Re}_{D}=250$ mit einer spannweitige Wellenlänge von etwa $y_{L}=1 D$ beobachtet (Zhang et al. 1995 [102], Brede et al. 1996 [15], Williamson 1996 [96]). Diese sekundäre Längswirbelstruktur bildet sich mit den von-Kármán-Wirbeln paarweise aus und umschliesst die von-Kármán-Wirbel schlangenförmig. Die Ursache ihrer Enstehung wird in der Scherschichtinstabilität gesehen (Lin et al. 1995 [45]). Für den Rey- 
noldszahlbereich $R e_{D}=360$ bis $R e_{D}=1300$ dominieren die von-Kármán-Wirbel und die Längswirbel der B-Mode die Wirbelstruktur im Nachlauf. Bekanntermaßen nimmt in diesem Reynoldszahlbereich die Länge des Rezirkulationsgebietes zu, um bei $R e_{D}=1300$ die größte Ausdehnung zu erfahren. Mit weiter zunehmender Reynoldszahl nimmt diese Länge wieder ab und es werden konvektive Instabilitätswellen in den freien abgelösten Scherschichten beobachtet. Analog zu den Tollmien-Schlichting-Wellen (TS-Wellen) in der ebenen Plattengrenzschichtströmung entdeckte Bloor (1964) [12] und untersuchten Bloor und Gerrard (1966) [13] eine sich wellenförmig ausbreitende Instabilität in der abgelösten Scherschicht in einem Reynoldszahlbereich der Größenordnung von etwa $\operatorname{Re}_{D}=10^{3}$ bis $10^{4}$. Diese Wellen entsprechen der Kelvin-Helmholtz-Wellen ( $\mathrm{KH}$-Wellen), die sich in freien abgelösten instabilen Scherschichten ausbilden (s.a. Rockwell 1983 [69]). Da diese Wellen nun bei den beiden wechselseitig abgelösten Scherschichten zu beobachten ist, werden diese KH-Wellen auch Bloor-Gerrard-Wellen (BG-Wellen) genannt (Unal und Rockwell 1988 [92], Zdravkovich 1997 [100]). Sie weisen in ihrer konvektiven Entwicklung ein exponentielles Anwachsen der Amplitude auf und sind gegenüber spannweitigen Störungen instabil. Dies führt zur Ausbildung spannweitig kleinskaliger Längswirbelstrukturen, die sich um die von-Kármán-Wirbel winden und die Transition der freien abgelösten Scherschichten bewirken (Wei und Smith 1986 [94], Wu et al. [97]). Auch numerische Untersuchungen zeigen das Auftreten dieser Längswirbelstrukturen in diesem Reynoldszahlbereich (Mittal und Balachandar 1994 [50], 1995 [51]). Der Ort der Transition in den freien abgelösten Scherschichten und die Ausdehnung des Rezirkulationsgebietes unterliegt dabei einem Zusammenhang. Schiller und Linke (1933) [80] fanden durch statische Druckmessungen mit einer Pitotrohrsonde im Nah-Nachlauf des Zylinders, dass der Ort der Transition in der Scherschicht mit zunehmender Reynoldszahl stromauf wandert und bei $R_{D}=10^{4}$ sich nur noch $x=0,7 D$ stromab von der Zylindermitte befindet. Der Widerstandsbeiwert wird dabei mit zunehmender Reynoldszahl in dem Maße größer, wie der Saugdruck im Heck des Zylinders durch die Verbreiterung des Rezirkulationsgebietes zunimmt. Die Länge des Rezirkulationsgebietes nimmt dabei aber stetig ab. Am Ende des von Roshko (1993) [70] so genannten SchillerLinke-Bereiches wird ein maximaler Widerstandsbeiwert von etwa $C_{W}=1,2$ bei einer Reynoldszahl von etwa $\operatorname{Re}_{D}=5^{\times} 10^{4}$ erreicht und die Länge des Rezirkulationsgebietes beträgt etwa $1 D$. Mit weiterer Erhöhung der Reynoldszahl wird das Rezirkulationsgebiet hinter dem Zylinder nun nicht mehr kleiner und die Transition in den freien Scherschichten wandert vermutlich auch nicht mehr weiter stromauf (Brede 2000 [16]). Ist die Oberfläche des Zylinders hinreichend glatt, verharren die Werte des Widerstandsbeiwertes und der Strouhalzahl für einen großen Reynoldszahlbereich auf ihrem jeweiligen Wert. Erst bei einer Reynoldszahl von etwa $R e_{D}=$ $2,0 \times 10^{5}$ setzt der Transitionsprozess der gerade ablösenden Grenzschicht ein. Dieser Prozess ist mit zum Teil abrupten Veränderungen im Widerstandsbeiwert (Wieselsberger 1921 [95]), im Basisdruckbeiwert (Flachsbart 1932 [28]), und auch im dynamischen Verhalten, z.B. ablesbar an der Strouhalzahl der Wirbelablösefrequenz, verbunden (Relf und Simmons 1924 [65], Delany und Sorrensen 1953 [23], Roshko 1961 [71]).

Als Erster deutete Prandtl 1926 [63] dieses Phänomen des drastischen Widerstandsabfalls für die Kugel- und in Analogie für die Zylinderströmung richtig. Während bei kleineren Reynoldszahlen unter $\operatorname{Re}_{D}=2,0^{\times} 10^{5}$ die Grenzschichtströmung laminar ist und bei einem Azimutwinkel von etwa 75 Grad ablöst, ist sie für größere Reynoldszahlen $R e_{D} \geq 3,5^{\times} 10^{5}$,,wirbelig, was zur Folge hat, daß der Keil von stagnierender Flüssigkeit hinter der Ablösestelle von den Wirbeln weggespült wird, wodurch die Strömung sich wieder an die Kugeloberfläche anlegt und erst wei-

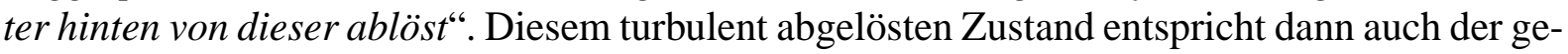


ringe Widerstandsbeiwert. Zum Beweis dieser These benutzte Prandtl einen dünnen Drahtreif, der knapp stromauf der laminaren Ablösestelle um die Kugel gelegt wurde. Der ,Stolperdraht“ bewirkte dabei ein Turbulentwerden der Grenzschicht bei einer Reynoldszahl, wo ohne Drahtreif laminare Ablösung beobachtet wird. In den Rauchvisualisierungen ist deutlich der Unterschied der Wirkung des dünnen Drahtreifes auf die Grenzschichtströmung und des Nachlaufs zu erkennen (Bild 1.5).
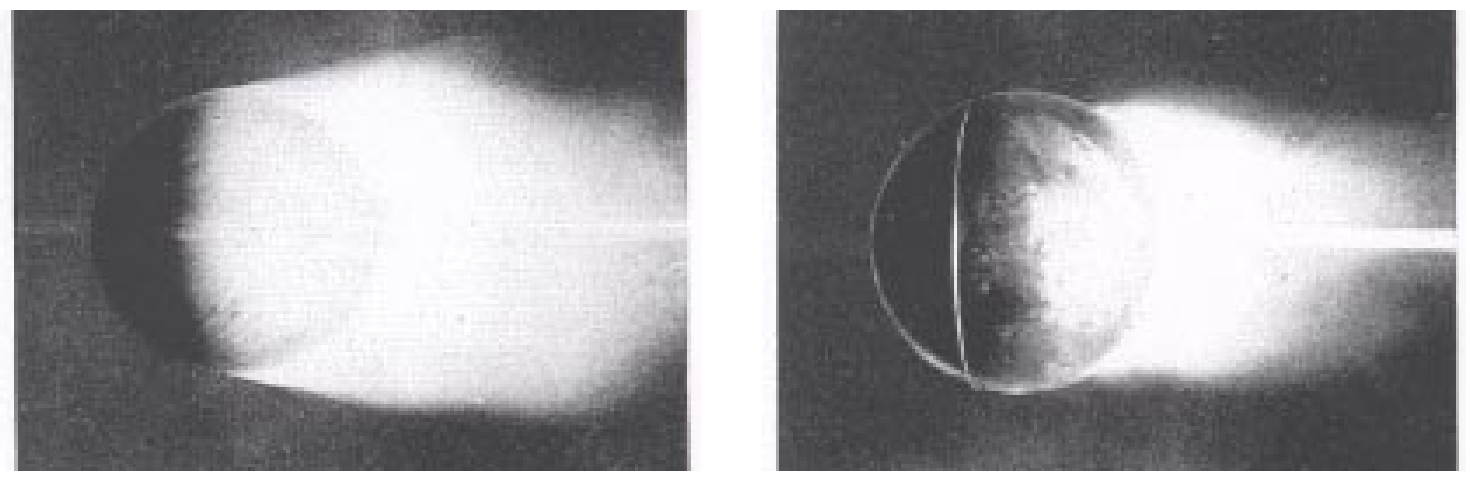

Bild 1.5 Rauchvisualisierungen der Kugelumströmung ohne (links) und mit dünnem Drahtreif, dem sog. ,Stolperdraht“ (rechts). Ohne ,Stolperdraht“ löst die Grenzschicht laminar ab, mit „Stolperdraht" wird sie turbulent und bewirkt eine deutliche Verringerung der Breite des Ablösegebietes (Prandtl 1926 [63]).

Mit dem Abfall des Widerstandsbeiwertes wurde im kritischen Bereich für einen kleinen Reynoldszahlausschnitt aber auch ein von Null beträchtlich verschiedener Auftriebsbeiwert gefunden. Da dieser Effekt nur für einen sehr kleinen Reynoldszahlausschnitt festgestellt wurde, wurde dieser anfänglich noch nicht richtig verstanden (Krämer 1964 [40]). Später beobachtete auch Bearman (1969) [7] diesen asymmetrischen abgelösten Strömungszustand bei $R_{D}=$ $3,7 \times 10^{5}$, verknüpft mit einer diskontinuierlichen Änderung im Basisdruckbeiwert (Bild 1.6 links). Bearman mutmaßte zunächst aufgrund spannweitiger Druckverteilungsmessungen des Basisdruckes und bewies schließlich mit Hilfe von Anstrichbildern, dass sich nur auf einer Seite, der Oberseite des Zylinders, eine Ablöseblase ausgebildet hat, die zu diesem asymmetrischen Strömungszustand führt. Eine detaillierte Beschreibung von den Änderungen im Widerstandsbeiwert und in der Strouhalzahl (Bild 1.6 rechts) liefert darüberhinaus Schewe (1983) [75]. Mit Kraftmessungen fand er in Abhängigkeit von der Reynoldszahl die Existenz von zwei diskontinuierlichen Übergängen, die er mit „Transition $A$ “ (kurz: „Tr $A^{\text {") }}$ ) und mit

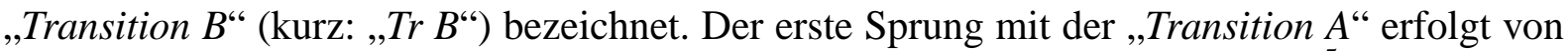
etwa $C_{W}=0,7$ auf $C_{W}=0,5$ und $S r=0,19$ auf $S r=0,33$ bei etwa $R e_{k r i t, A}=3,2^{\times} 10^{5}$. Damit bildet sich ein asymmetrischer Strömungszustand aus, was in einem deutlichen, von Null verschiedenen mittleren Auftriebsbeiwert von etwa $\left|C_{L}\right|=1,0$ erkennbar ist. Dieser Zustand erweist sich als bistabil, so dass der Auftriebsbeiwert mit beiden Vorzeichen beobachtet werden kann. Während bei hinreichend glatter Zylinderoberfläche sich jeweils einer der beiden möglichen asymmetrischen Zustände auf natürliche Weise unbestimmt einstellten, kann auch mit Hilfe einer künstlich eingebrachten Störung stromauf der Ablösung der eine oder der andere asymmetrische Zustand bei Reynoldszahlen knapp unterhalb der kritischen Reynoldszahl $R e_{k r i t, A}$ erzwungen werden (Schewe 1986) [77]. Mit der „Transition B“, dem zweiten diskontinuierlichem Abfall von etwa $C_{W}=0,45$ auf $C_{W}=0,22$ und von $S r=0.3$ auf $S r=0.5$ bei $\operatorname{Re}_{k r i t, B}=3,5^{\times} 10^{5}$ 
löst die Strömung wieder symmetrisch ab, so dass sich jetzt auf jeder Seite des Zylinders eine transitionelle Ablöseblase formiert hat (Bild 1.6). Darüber hinaus konnte Schewe (1983) [75] zeigen, dass das Durchlaufen des asymmetrischen Strömungszustandes im kritischen Reynoldszahlbereich auf einer subkritische Verzweigung basiert und die Übergänge in Abhängigkeit von Reynoldszahl-,,Richtung“ betrachtet werden müssen. Mit aufsteigender Reynoldszahl werden die bekannten Übergänge „Transition $A$ “ und „Transition $B$ “ beobachtet, während mit absteigender Reynoldszahl die „Transition $B *$ * und „Transition $A$ * “ erfolgen.
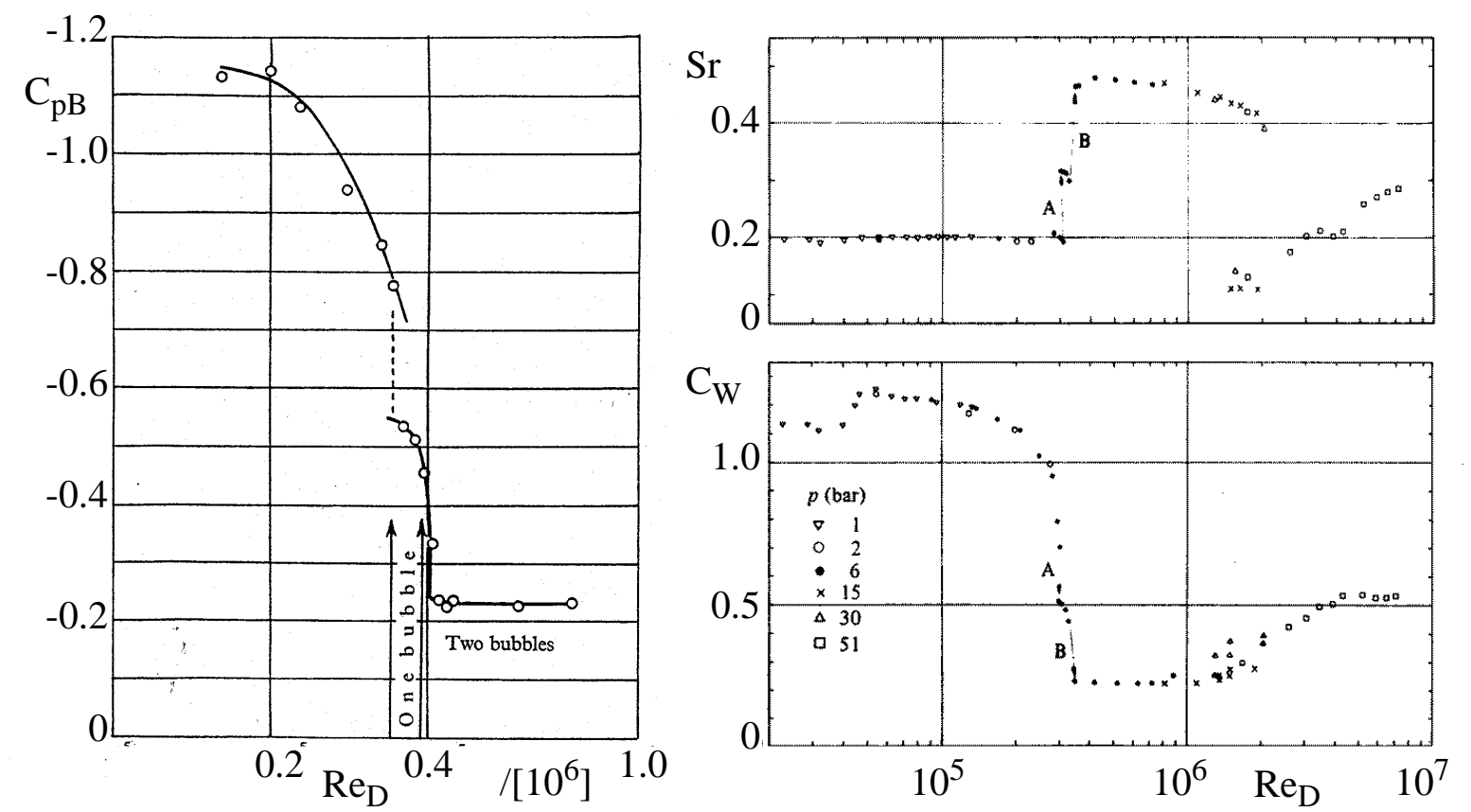

Bild 1.6 Basisdruckmessungen von Bearman (1969) [7] (links) und Verhalten des Widerstandsbeiwertes und der Strouhalzahl der Wirbelablösefrequenz in Abhängigkeit von der Reynoldszahl beim laminar-turbulenten Übergang aus Schewe (1983) [75] (rechts)

Mit diesem kritischen Verhalten der abgelösten transitionellen Zylinderströmung ist die Transition der ablösenden Grenzschicht noch nicht abgeschlossen. Mit der „Transition B“ wird ein neues Plateau mit minimalem Widerstandsbeiwert von etwa $C_{W}=0,22$ und mit einer Strouhalzahl der Wirbelablösefrequenz von etwa $S r=0,5$ erreicht. Erst bei größeren Reynoldszahlen ab $R e_{D}=1,0^{\times} 10^{6}$ wächst der Widerstandsbeiwert wieder an. Eine dominante Ablösefrequenz ist im Fourierspektrum der Querkraft immer schwerer zu identifizieren. Niederfrequente Anteile wachsen an und der Widerstandsbeiwert nimmt nach Schewe (1983) [75] sogar für eine Reynoldszahl mehrere Werte an, was auf eine unterschiedliche Strömungsstruktur der Wirbelablösung hindeutet (Dallmann und Schewe 1987 [20]). Tatsächlich wurden für Reynoldszahlen oberhalb der kritischen Reynoldszahl erste Hinweise für die Ausbildung von spannweitig periodisch ablösenden Wirbelstrukturen mit Hilfe von Seidenfäden und Hitzdrahtmessungen im Nah-Nachlauf des Zylinders (Humphreys 1960 [37]) oder mit Hilfe von Anstrichbildern (Dallmann und Schewe 1987 [20], Schewe 2000 [78]) nachgewiesen. Die spannweitigen Längen einer sog. Zelle dieser Ablösestruktur sind ähnlich und betragen $y_{L}=1,7 D$ bis $y_{L}=2,1 D$. Dallmann und Schewe (1987) [20] postulierten, dass diese großskaligen Längswirbelstrukturen verknüpft sind mit der Entstehung der kleinskaligen Längswirbelstrukturen in den freien Scher- 
schichten. Numerische Untersuchungen von Tamura et al. (1990) [88] unterstützen diese Vermutung. Darüberhinaus zeigen experimentelle (Owen et al. 2000 [58]) sowie numerische Untersuchungen für kleine Reynoldszahlen (Darekar et al. 2000 [22], Meneghini et al. 1992 [47]), dass durch Entstehung von großskaligen Längswirbelstrukturen die von-Kármán-Wirbel unterdrückt und somit eine erhebliche Widerstandsreduktion erreicht werden kann. In diesem Zusammenhang wurden auch Instabilitätsuntersuchungen an einer laminaren Ablöseblase durchgeführt (Rist et al. 1996 [68]) Während erste Ergebnisse keine Beeinflussungsmöglichkeit der laminare Ablöseblase durch stromauf künstlich eingebrachte Störungenen hervorbrachten, zeigen andere (Theofilis et al. 2000 [90], Barkley et al. 1996 [6]), dass die Ablöseblase instabil gegenüber sog. globalen spannweitigen Moden sein kann. Somit könnte das spannweitige Instabilitätsverhalten der Ablöseblase Ausgangspunkt für die Ausbildung von spannweitig periodischen Längswirbelstrukturen sein.

Erst wenn der Widerstandsbeiwert etwa den Wert von $C_{W}=0,5$ bei Reynoldszahlen ab etwa $R e_{D}$ $=3,0^{\times} 10^{6}$ erreicht, wird wieder ein periodisches Ablösen der Strömung festgestellt, was in Spektren von Hitzdrahtsignalen aus dem Nachlauf (z.B. Roshko 1961 [71]) und in der Auftriebskraft (Schewe 1983 [75]) zu beobachten ist. Die Strouhalzahl der Wirbelablösefrequenz ist dabei etwas größer als $S r=0,2$. Mit diesen Reynoldszahlen - oberhalb von etwa $R e_{D}=5^{\times} 10^{6}$ - ist der Zustand erreicht, wo erstmalig die anliegende Grenzschichtströmung vor der Ablösung turbulent ist. Der laminar-turbulente Übergang vollzieht sich nun in der am Zylinder anliegenden Grenzschicht, wobei analog zum Schiller-Linke-Bereich der Ort der Transition mit zunehmender Reynoldszahl sich immer weiter stromauf verlagert.

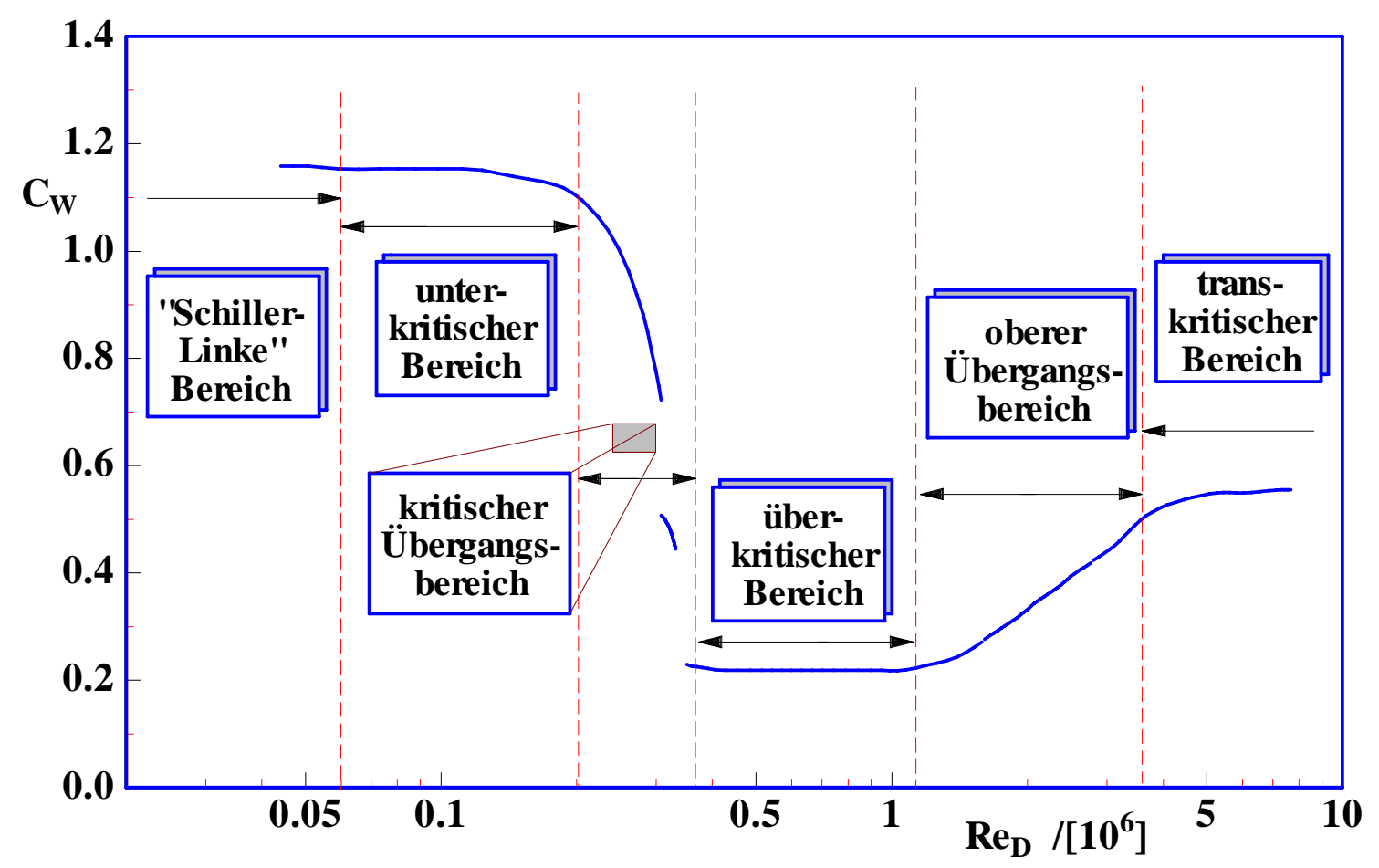

Bild 1.7 Charakterisitischer Verlauf des Widerstandsbeiwertes beim laminar-turbulenten Übergang der ablösenden Zylindergrenzschicht mit Definition der Reynoldszahlbereiche für die vorliegende Arbeit in Anlehnung an Roshko (1961) [71] 
Um die unterschiedlichen Phänomene des laminar-turbulenten Überganges der ablösenden Zylindergrenzschicht übersichtlich beschreiben zu können, wurde als erstes von Roshko 1961 [71] eine Unterteilung in charakteristische Reynoldszahlbereiche (,characteristic ranges“) vorgeschlagen. Leider zeigt die weitere Entwicklung keine einheitliche Benutzung dieser Begriffe Morkovin 1964 [52], Achenbach und Heinecke 1981 [2] oder Niemann und Hölscher 1990 [55]). Andere führten gar neue Bezeichnungen, die zum Teil im Widerspruch zu den existierenden Beschreibungen stehen (Bearman 1969 [7], Roshko 1993 [72], Williamson 1996 [96]). Auch Zdravkovich (1997) [100] leistet hierzu keinen zufriedenstellenden Beitrag. Letztlich wird eine einheitliche Nomenklatur benötigt, die auch im Konsens zu Begriffen zu der Beschreibung der Transition z.B. bei der Kugelumströmung (Taneda 1978 [89], Achenbach 1979 [1]) steht. Daher werden in der vorliegenden Arbeit die Bezeichnung der Reynoldszahlbereiche von Roshko (1961) [71] übernommen (Bild 1.7). Eine Übersicht über die Einteilung und Bezeichnung der Reynoldszahlbereiche der anderen oben zitierten Autoren sind im Anhang A.l Re-Bereiche beim laminar-turbulenten Übergang der Zylindergrenzschicht angegeben.

\subsection{Vorgehensweise}

Prandtl leistete mit seinem ,Stolperdraht"-Experiment einen wichtigen Beitrag zur Erklärung des unterschiedlichen Verhaltens laminarer und turbulenter ablösender Grenzschichten. Gleichzeitig gibt er den Weg frei, über die Beeinflussung von abgelösten Strömungen das transitionelle Strömungsverhalten grundsätzlich zu verstehen. Die Natur verhält sich so, dass mit Änderung des Ordnungszustandes der Strömung große Änderungen im Widerstands- und im dynamischen Verhalten verbunden sind. Daraus erwächst das Interesse an Verfahren zur Widerstandsverringerung, was durch künstliche Änderung des Ordnungszustandes, d.h. der Struktur der Strömung erreicht wird.

Wie oben beschrieben, wird das Ablöseverhalten der Zylinderströmung maßgeblich durch das Instabilitätsverhalten der Scherschichten bestimmt. Bei der Transition des Nachlaufs und der freien abgelösten Scherschichten bedingt zunächst die Instabilität gegenüber spannweitig periodischen Störungen und dann die Ausbildung von spannweitig periodischen Längswirbelstrukturen den Übergang in die Turbulenz. Auch bei der Transition der ablösenden Grenzschicht werden mit dem Übergang in den transkritischen Bereich großskalige, spannweitig periodische Wirbelstrukturen gefunden. Auch hier handelt es sich um Längswirbelstrukturen. Die Turbulenzforschung sucht damit Antworten auf Fragen wie: was macht eine laminare Grenzschicht turbulent, welche Mechanismen sind für den laminar-turbulenten Übergang verantwortlich und welche Strukturen sind damit verbunden und schließlich, wie können diese beeinflusst oder gesteuert werden?

Hinsichtlich der Strömungsbeeinflussung bzw. -steuerung ist unser erstes Ziel, solche Strömungsstrukturänderungen, d.h. räumliche und zeitliche Symmetrieänderungen einer Strömung zu identifizieren, die Widerstandsänderungen begleiten. In einem zweiten Schritt wird es darum gehen solche Struktur- und Symmetrieänderungen künstlich herbeizuführen.

In einem ersten Teil der vorliegenden Arbeit im Kapitel 3 Ergebnisse zur natürlich abgelösten Zylinderströmung wird das dynamische und räumliche Ablöseverhalten der Zylindergrenzschicht beginnend im unterkritischen bis anfänglich transkritischen Reynoldszahlbereich untersucht. Insbesondere in den kritischen Bereichen werden mit einer hohen Auflösung in der Reynoldszahl die Wirbelstrukturen identifiziert, die entweder für den Übergang verantwortlich sind bzw. ihn begleiten. Dabei wird versucht, zeitliche und spannweitige Skalen anzugeben, um 
damit die Voraussetzungen für eine geeignete künstliche Anregung der Grenzschicht zu schaffen.

In dem zweiten Teil, im Kapitel 4 Ergebnisse zum Verhalten der angeregten Zylindergrenzschicht, werden verschiedene Beeinflussungs- bzw. Steuerungsverfahren angewendet, um Instabilitäten in der Grenzschicht anzuregen, die bestimmte Wirbelstrukturen hervorbringen, um somit eine Widerstandsminderung z.B. im unterkritischen Bereich gezielt zu erreichen. Neben der Möglichkeit die Grenzschicht durch einen Stolperdraht turbulent zu machen, sie abzusaugen oder gar Fluid in die Scherschicht auszublasen, gibt es eine Vielzahl von weiteren Beeinflussungsverfahren oder -techniken. Gad-el-Hak (1996) [31] hat die Beeinflussungsstrategien in einer Systematik zusammengefasst und teilt diese in zwei Gruppen ein - in die passive und in die aktive Steuerung, wobei letztere in vorbestimmte (,predetermined“) und reaktive (,reactive“) Verfahren weiter untergliedert wird. Zu den reaktiven Verfahren gehören dabei die Rückkopplungsverfahren wie adaptive Algorithmen als auch Steuerungen, die auf Modellgleichungen basieren.

Für die Beeinflussung der laminaren Ablösung kann z.B. zunächst das Geschwindigkeitsprofil einer zweidimensionalen Grenzschicht betrachtet werden (Gad-el-Hak und Bushnell 1991 [30]). Durch eine passive Störung, wie z.B. durch Wirbelerzeuger oder Turbulenzgeneratoren, wird die laminare Anordnung der Scherschicht zerstört (Morkovin 1984 [53]). Bei geeigneter Form der Wirbelerzeuger bilden sich kleinskalige Wirbel aus, so dass die Grenzschicht turbulent wird und so weiter stromab an der Körperkontur anliegend bleibt (Lin et al. 1991 [44]). Bei der aktiven Steuerung durch Absaugen wird die noch anliegende Grenzschicht an der Wand beschleunigt. Das zuvor wendepunktbehaftete Geschwindigkeitsprofil wird in Wandnähe voller, so dass die Ablösung zunächst verhindert werden kann. Auch durch Kühlen der Wand bei Umströmung in Gasen lässt sich theoretisch eine Ablösung verzögern. Für die Beschreibung der Wirkung von zeitlich periodischen oder dreidimensionalen Störungen auf die ablösende Grenzschicht reicht dieses einfache Modell der „Geschwindigkeitsprofil-Veränderer“ nicht mehr aus. Eine umfassenderes Werkzeug zur Umsetzung von Beeinflussungs- bzw. Steuerungsverfahren ist durch Instabilitätsanalysen (Huerre und Monkewitz 1990 [38]) gepaart mit energetischen Betrachtungen (Wu et al. 1991 [98]) gegeben. Im unterkritischen Reynoldszahlbereich prägen die spannweitig homogen ablösenden Querwirbel das Verhalten der Zylinderströmung. Wird dieser kohärenten Wirbelstruktur Energie entzogen, kann diese in ihrer Ausbildung unterdrückt werden. Eine passive Methode bei laminarer Ablösung ist die Einführung eines dünnen Drahtes in den Nah-Nachlauf des Zylinders (Strykowski und Sreenivasan 1990 [86], Fey 1997 [27]). Durch die lokale Einbringung einer künstlichen Störung, wie einem Stift auf der Oberfläche des Zylinders stromauf der Ablösung im kritischen Reynoldszahlbereich wird der symmetrisch abgelöste Zustand instabil. Bereits bei kleineren Reynoldszahlen kann somit die „Transition A“ hervorgerufen werden (Schewe 1986 [77]). Mit der akustischen Anregung der von Kármán'schen Wirbelstraße in der Nähe der Ablösefrequenz wird eine deutlich erhöhte spannweitige Kohärenz der Ablösung im Schiller-Linke-Bereich beobachtet (Blevins 1985 [11]). Dieses Phänomen kann auf das Einkopppeln (,Lock-in“) der akustischen Störwellen in das zeitlich periodische Ablösen der Grenzschichten zurückgeführt werden. Wird die Anregungsfrequenz auf etwa $S r_{E}=1$ erhöht, werden die freien abgelösten instabilen Scherschichten angeregt (Hsiao et al. 1991 [36]). Insbesondere bei künstlicher Störung in der Nähe der laminaren Ablösung wird eine stromab veränderte Druckverteilung erhalten. Das Ergebnis ist ein mittlerer Auftrieb und eine signifikante Widerstandsreduktion. Darüber hinausgehend konnten Owen et al. (2000) [58] 
sowie Darekar und Sherwin (2000) [22] eine deutliche Beeinflussung des instationären Strömungsverhaltens abgelöster Platten- und Zylinderumströmungen durch stationäre dreidimensionale, spannweitig periodische Verformung der Körperoberfläche erreichen. Es scheint somit möglich im unterkritischen Bereich dreidimensionale Instabilitäten durch spannweitig periodische Variation der Körperkontur und somit des Druckes anzuregen (Bushnell 1992 [18]). Die Folge ist die Generierung dreidimensionaler Wirbelstrukturen, die das Ablöseverhalten verändern und zu einer Widerstandsreduktion führen. Um eine effiziente Beeinflussung realisieren zu können, ist es notwendig die räumlichen Skalen der zu erzeugenden Wirbelstrukturen zu kennen. Für die kleinskaligen Längswirbelstrukturen der freien abgelösten instabilen Scherschichten im Schiller-Linke-Bereich treten spannweitige Wellenlängen in Abhängigkeit von der Reynoldszahl zwichen zwischen $\lambda_{B G}=0,8 D$ bis $\lambda_{B G}=0,3 D$ auf (Wu et al. 1995 [97]). Die großskaligen zellularen Wirbelstrukturen beim Übergang in den transkritischen Bereich besitzen hingegen spannweitige Längen von etwa $y_{L}=1,7 D$ bis $y_{L}=2,1 D$ (Humphreys 1960 [37], Dallmann und Schewe 1987 [20]).

Im Kapitel 4.1 Stationäre Störung mit spannweitig verteilten Wirbelerzeugern wird das Verhalten der Grenzschicht auf stationäre Anregung durch spannweitig verteilte Wirbelerzeuger getestet. Eine andere stationäre, aber aktive Störung der Grenzschicht erfolgt mit spannweitigperiodischem kontinuierlichem Absaugen. Im Vergleich mit dem spannweitig homogenen Absaugen wird das Ablöseverhalten in Kapitel 4.2 Stationäre Störung der Grenzschicht durch Absaugen diskutiert. Mit der instationären und spannweitig-periodischen Anregung der Grenzschicht in Kapitel 4.3 Instationäre Störung der Grenzschicht durch akustische Anregung wird die Idee verfolgt, eine für den laminar-turbulenten Übergang dominante Instabilität anzuregen. Auch hier wird der spannweitig homogene Fall mit und ohne zeitabhängige Strömungsanregung für den Vergleich herangezogen.

Um sowohl das Ablöseverhalten der nicht künstlich beeinflussten Zylinderströmung studieren als auch die unterschiedlichen Beeinflussungsverfahren anwenden zu können, wurden verschiedene Zylindermodelle und mehrere Messverfahren und Techniken eingesetzt. Auf die Durchführung der Experimente in einem Windkanal des Deutsch-Niederländischen Windkanalverbunds $(D N W)$ in Göttingen beim Deutschen Zentrum für Luft- und Raumfahrt e.V. (DLR) und die Anwendung der Versuchstechnik sowie die verwendeten Zylindermodelle wird im $\mathrm{Ka}$ pitel 2 Experimenteller Aufbau und Versuchsdurchführung eingegangen. 


\section{Experimenteller Aufbau und Versuchsdurchführung}

Die experimentellen Untersuchungen werden im Hochdruckwindkanal Göttingen durchgeführt, so dass der relevante Reynoldszahlbereich von etwa $\operatorname{Re}_{D}=5^{*} 10^{4}$ bis $R e_{D}=5 \times 10^{6}$, also der unterkritische bis anfänglich transkritische Bereich des laminar-turbulenten Übergangs der Zylindergrenzschicht, erfasst wird. Um das zeitlich gemittelte wie auch das dynamische Verhalten der abgelösten Zylinderströmung zu beschreiben, können verschiedene Messverfahren und Messtechniken eingesetzt werden (Eckelmann 1997 [24]). Einen Überlick über das zeitlich gemittelte dreidimensionale Ablöseverhalten wird mit Hilfe der Anstrichtechnik erhalten, während instationäre Kraft- und statische als auch dynamische Druckmessungen die Grundlage für die Bestimmung von Beiwerten und der Strouhalzahl der periodischen Wirbelablösung liefern. Darüberhinaus soll durch die Einbringung verschiedener künstlicher Störungen in die Zylindergenzschicht das Ablöseverhalten beeinflusst werden. Ein passives und aktive Anregungsverfahren kommem dabei zur Anwendung.

\subsection{Hochdruckwindkanal Göttingen}

Mit dem Hochdruckwindkanal Göttingen (HDG, Bild 2.1) steht seit 1981 ein mit hohem Überdruck betreibbarer Windkanal zur Verfügung (Försching et al. 1981 [29]). Die inkompressible Luftströmung zeichnet sich durch einen geringen Turbulenzgrad von etwa $0,1 \%$ bis $0,3 \%$ in Abhängigkeit vom Massenstrom aus.

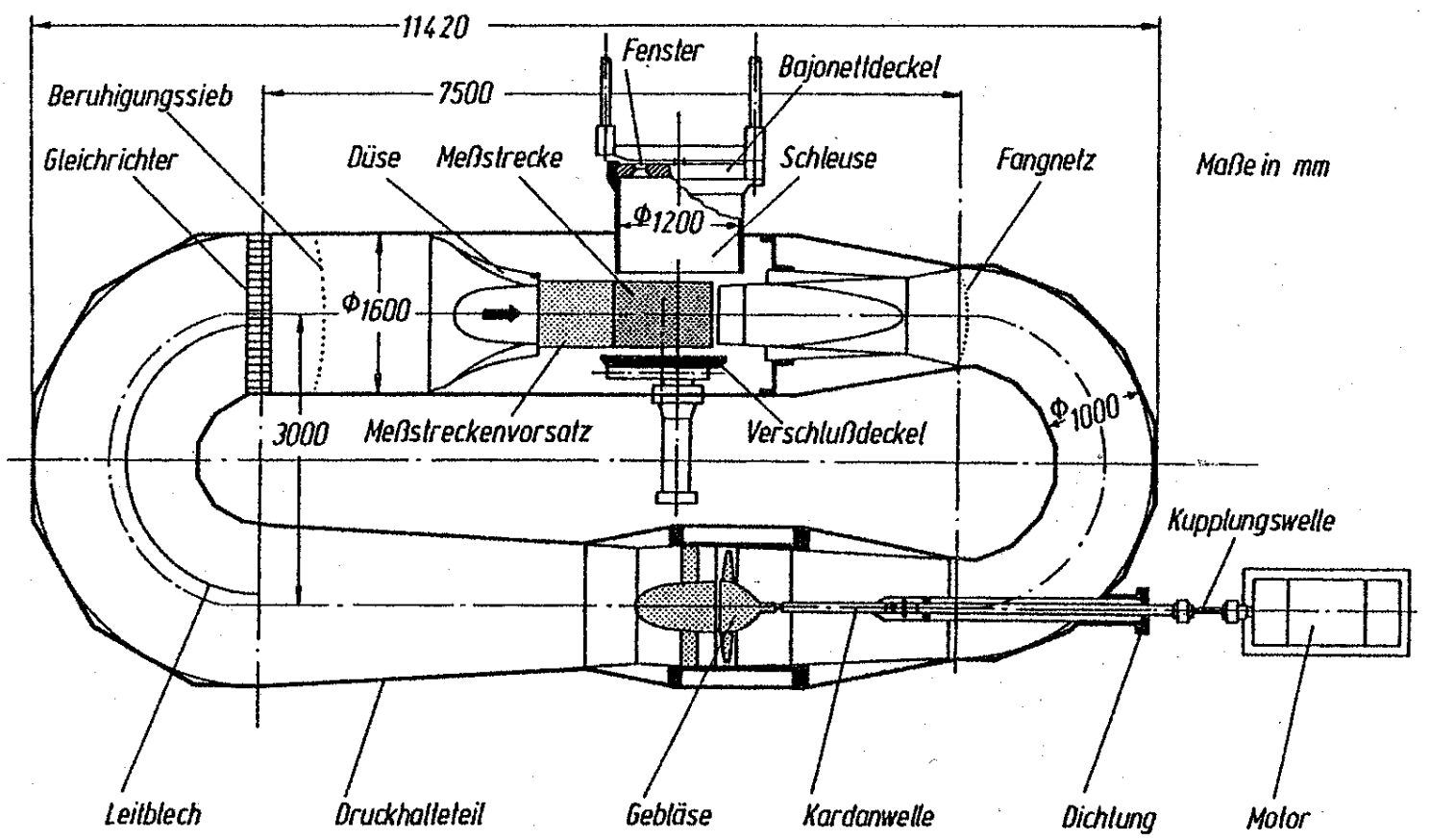

Bild 2.1 Schematische Darstellung des Hochdruckwindkanals Göttingen (HDG) (aus Försching et al. 1981 [29]) 
Die Bauweise des Kanals ermöglicht den Einsatz einer geschlossenen Messstrecke und Geschwindigkeiten bis zu $U_{\infty, \max }=35 \mathrm{~m} / \mathrm{s}$. Die Messstrecke hat einen quadratischen Querschnitt von 0,6 $0,6 \mathrm{~m}^{2}$. Mit einem Durchmesser von $D=60 \mathrm{~mm}$ des Zylindermodells wird ein Streckungsverhältnis von $L / D=10$ erreicht und eine Versperrung von $B=10 \%$ in Kauf genommen. Das Streckungsverhältnis ist prinzipiell groß genug, so dass dreidimensionale Ablösungen aufgrund geometriebedingter Randstörungen ausgeschlossen werden können (Norberg 1994 [56], Morsbach 1967 [54]). Die Größe der Versperrung bewirkt andererseits eine, gegenüber der Potentialströmung an den Schultern des Zylinders, stärker beschleunigte Strömung (Richter und Naudascher 1976 [67]), so dass die Werte für Anströmgeschwindigkeit und Beiwerte entsprechend z.B. nach Allen und Vincenti (1944) [3] zu korrigieren sind, um mit Ergebnissen aus anderen Windkanälen vergleichen zu können. Die für die Experimente wesentliche Eigenschaft des Windkanals ist, die Luft bis zu 100 bar unter Druck setzen zu können. Damit kann die Dichte und somit auch die Reynoldszahl innerhalb von zwei Zehnerpotenzen variiert werden, da die dynamische Viskosität der Luft sich nur gering in Abhängigkeit vom Druck ändert. Bei konstant gehaltenem Betriebsdruck im Windkanal kann die Reynoldszahl innerhalb einer Größenordnung verändert werden.

Der Einsatz verschiedener Messverfahren oder Messtechniken ist im HDG aufgrund des massiven Aufbaus der Messstrecke und aufgrund der geforderten Druckbeständigkeit der messtechnischen Apparaturen beschränkt. Die Messstrecke selbst kann durch das Schleusensystem aus dem Windkanal herausgenommen werden, wobei der Betriebsdruck im Kanal erhalten bleibt.

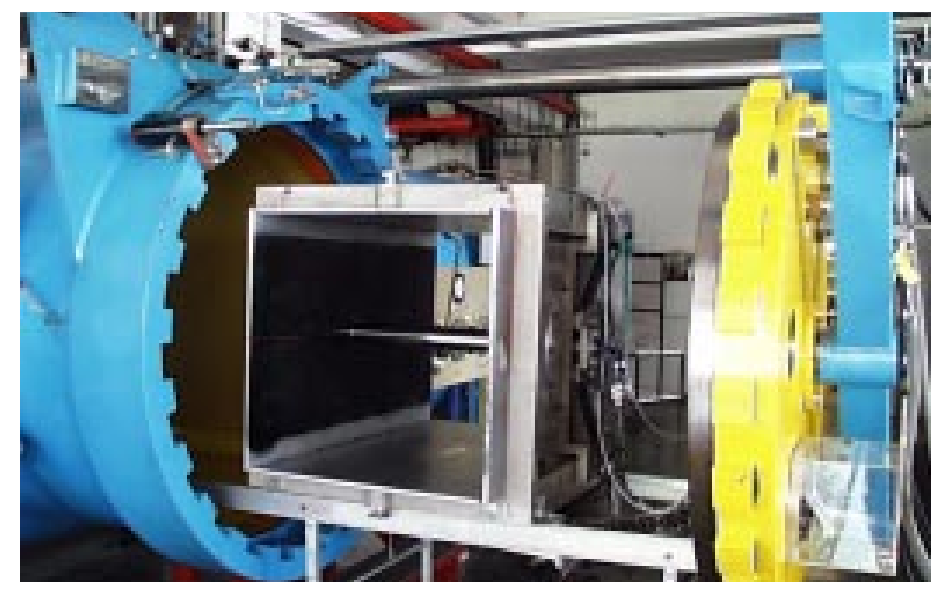

Bild 2.2 Messstrecke mit der Piezowaage und „Referenz"-Zylindermodell außerhalb des Windkanals bei geöffneter Schleuse. An der rechten Außenwand der Messstrecke ist die eine Hälfte der Piezowaage zu sehen.

Für die Messungen wurden zwei unterschiedliche Messstrecken verwendet. Eine Messstrecke ist mit dem vierelementigen piezoelektrischen Kraftaufnehmersystem, im folgenden auch kurz Piezowaage genannt, ausgerüstet (Bild 2.2). Sie ist so konfiguriert, dass Kraftwirkungen über Spannelemente an den seitlichen Enden des Zylinders auf die Piezokristalle übertragen werden. Spannelemente und Piezoelemente befinden sich dabei außerhalb der Messstrecke, so dass die Zylinderströmung nicht beeinträchtigt wird. Eine andere Messstrecke ist mit einer drehbar gelagerten Spannvorrichtung ausgestattet. Über eine elektronische Schrittmotorsteuerung lassen sich verschiedene Drehwinkel anfahren, wobei der Drehwinkelbereich bezogen auf eine Referenzposition etwa $\Delta \varphi_{D r}= \pm 35$ Grad beträgt. Weiterhin besteht die obere Abdeckung dieser Messstrecke aus einer optisch-durchsichtigen Glasplatte. Für eine erste Anwendung der An- 
strichtechnik war dies von Vorteil, da das Fließverhalten der Farb-Öl-Dispersion auf der Zylinderoberfläche beim Anfahren des Windkanals beobachtet werden konnte (s.a. 2.3.4 Anstrichbilder).

\subsection{Zylindermodelle}

Für die Durchführung der Experimente im $H D G$ kamen drei unterschiedlich instrumentierte Zylindermodelle mit einem Außendurchmesser von $D=60 \mathrm{~mm}$ zum Einsatz, um unterschiedlichen experimentellen Anforderungen und Aufgabenstellungen $\mathrm{zu}$ genügen. Das erste Zylindermodell, das ,Referenz"-Zylindermodell wird benutzt, um das Verhalten von Kraft- und Druckbeiwerten in Abhängigkeit von der Reynoldszahl für eine Zylinderströmung mit ,glatter“ Oberfläche zu bestimmen. Wie die Oberfläche hierfür beschaffen sein muss, wird später noch erörtert. Ein zweites Zylindermodell, das „Passiv“-Zylindermodell ist mit Wirbelerzeugern, als passive Aktuatoren, versehen und dient sowohl für die Untersuchung der Wirkung von stationären Störquellen auf die abgelöste Zylinderströmung als auch für die Anfertigung von Anstrichbildern. Das dritte, aufwendigste und am häufigsten benutzte Zylindermodell ist das „Aktiv“Zylindermodell. Neben der stationären und instationären Erfassung des statischen Druckes auf der Zylinderoberfläche hat es die Funktion, dass über ein entsprechend konstruiertes Aktuatormodul die verschiedenen Beeinflussungsverfahren zur Anregung der ablösenden Grenzschicht angewendet werden können.

\subsection{1 ,Referenz"-Zylindermodell}

Mit dem „Referenz“"Zylindermodell wird das zeitlich gemittelte und dynamische Verhalten der natürlich abgelösten Zylinderströmung untersucht. Neben der Messung von Widerstands- und Auftriebskraft mit der Piezowaage wird über eine Reihe von Druckbohrungen längs der Spannweite des Zylinders (Bild 2.3) das spannweitige Verhalten des Basisdruckes ermittelt. Dazu werden die Druckbohrungen auf einen Azimutwinkel von $\varphi_{P}=180$ Grad gestellt.

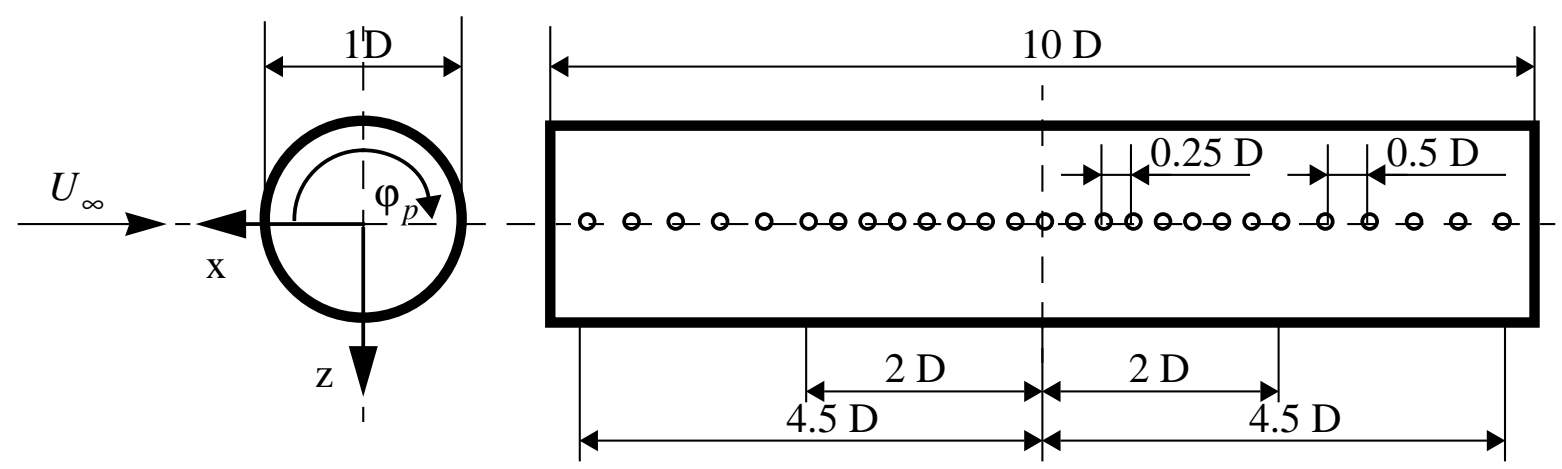

Bild 2.3 Schematische Darstellung des „Referenz“"Zylindermodells mit einer Reihe von Druckbohrungen

Damit das Ablöseverhalten durch Störungen auf der Oberfläche des Zylinders nicht beeinträchtigt wird, muss die Oberfläche hinreichend glatt sein. D.h., die mittlere Oberflächenrauigkeit $R_{Z}$ sollte kleiner als die Dicke der viskosen Unterschicht der Grenzschicht sein, so dass die Ungleichung $R_{Z} u^{+} / v<5$ erfüllt sein muss (Schlichting 1960 [81], S. 557). Zur annähernden Bestim- 
mung der Geschwindigkeit $u^{+}$wird über die Wandschubspannung vom vorderen Staupunkt bis zur Ablösestelle nach Messungen von Achenbach (1981) [2] gemittelt. Sie beträgt für unterkritische Reynoldszahlen etwa $\sqrt{\tau_{W} / \rho} \approx 1,3 \cdot U_{\infty} /\left(\sqrt[4]{R e_{D}}\right) \approx 1 \mathrm{~m} / \mathrm{s}$. Damit darf die mittlere Rauigkeit nicht größer sein als etwa $75 \mu \mathrm{m}$. Durch Schleifen und Polieren der Zylinderoberfläche wurde eine mittlere Rauigkeit von $R_{Z}<5 \mu \mathrm{m}$ erreicht, so dass damit die Zylinderoberfläche als glatt angesehen werden darf (siehe auch Feindt 1956 [26]). Weiterhin wurden die Druckbohrungen mit möglichst kleinen Durchmessern gefertigt. Aufgrund der Nutzung eines vorhandenen Modells gibt es zwei unterschiedlich ausgelegte Druckbohrungen mit einem Durchmesser von $d_{P}=0.5 \mathrm{~mm}$ (bei den Positionen $\mathrm{y}_{\mathrm{D}}=0,0 ; 0,5 ; 1,0 ; 1,5$ und 2,0) und sonst von $d_{P}=0,3 \mathrm{~mm}$. (Die kleineren Bohrungen wurden nachträglich eingebracht.) Der Unterschied besteht in der Ausführung. Die kleineren Bohrungen sind mit einer Fassung versehen, um diesen kleineren Durchmesser zu erreichen. Die Fassungen besitzen zwar einen Außendurchmesser von $d_{F}=0,6$ $\mathrm{mm}$, schließen aber mit der Zylinderoberfläche fast bündig ab. Genaugenommen besteht zwischen Fassungsrand und Zylindermantel eine minimale Versenkung. Experimentelle Untersuchungen von Hoerner (1958) [35] zeigen aber, dass die davon ausgehende Störung auf die Grenzschicht vernachlässigt werden darf.

Mit diesen Eigenschaften kann davon ausgegangen werden, dass die Grenzschicht am „Referenz"“-Zylindermodell durch Störungen an der Zylinderoberfläche nicht beeinträchtigt wird.

\subsection{2 ,Passiv"6-Zylindermodell}

Das „Passiv“-Zylindermodell wurde für die Anfertigung von Anstrichbildern und zur Studie der Wirkung von in einer Reihe längs der Spannweite angeordneten auf der Oberfläche aufgebrachten Störelementen, sog. Wirbelerzeugern, gefertigt. Die Wirbelerzeuger haben dabei die Form gleichseitiger Dreiecke mit einer Kantenlänge von $2 \mathrm{~mm}$, wobei die Dicke $1 \mathrm{~mm}$ beträgt. Sie befinden sich in einem Abstand von $\Delta y_{T G}=2 D$ an den spannweitigen Positionen $y_{T G}= \pm 1 D$ und $y_{T G}= \pm 3 D$ (Bild 2.4).

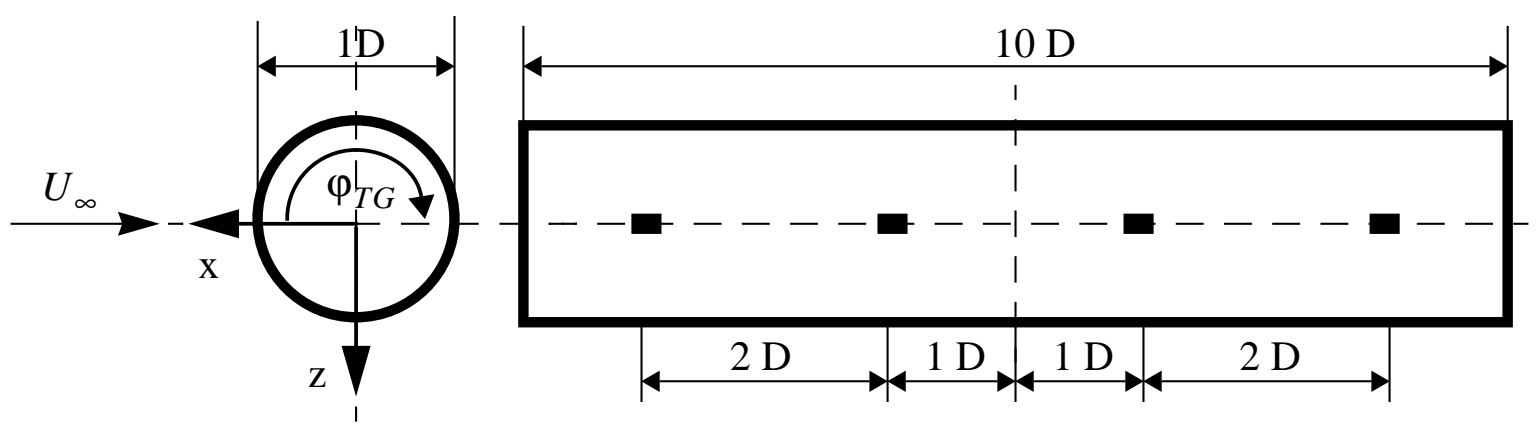

Bild 2.4 Schematische Darstellung des „Passiv“-Zylindermodells mit spannweitig verteilten Wirbelerzeugern im Abstand von $\Delta y_{T G}=2 D$.

Bei der Anfertigung von Anstrichbildern bei natürlich abgelöster Strömung wurden die Wirbelerzeuger in den Heckbereich des Zylinders, auf einen Azimutwinkel von $\varphi_{T G}=180 \mathrm{Grad}$ gedreht. Experimentelle Ergebnisse mit einer Trennplatte im Rezirkulationsgebiet zeigen, dass eine störende Wirkung auf das Ablöseverhalten ausgeschlossen werden kann. So wird z.B. bei einer Reynoldszahl von $R_{D}=5^{\times} 10^{4}$ bei Trennplatten ab einer Tiefe von $L_{T}=1 / 16 \mathrm{D}$ eine geringe Reduktion im Widerstandsbeiwert beobachtet (Apelt et al. 1975 [4], Hoerner 1958 [35]). 
Da die spannweitig lokal verteilten Wirbelerzeuger mit einer Länge von $L_{T G}=1 / 35 \mathrm{D}$ im Vergleich dazu entschieden kleiner sind, kann davon ausgegangen werden, dass wesentliche Störungen auf das Ablöseverhalten der Zylinderströmung ausgeschlossen werden können, wenn die Wirbelerzeuger im Heck des Zylinders bei $\varphi_{T G}=180 \mathrm{Grad}$ positioniert werden (Roshko 1961 [71]).

Hingegen ist die störende Wirkung der Wirbelerzeuger bei passiver Beeinflussung erwünscht und wird in Abhängigkeit von der Reynoldszahl untersucht. Dazu werden zwei Azimutpositionen benutzt. Die erste Position ist bei einem Azimutwinkel von $\varphi_{T G}=55 \mathrm{Grad}$, um stets die anliegende noch laminare Grenzschicht zu stören. Die zweite Position bei $\varphi_{T G}=80 \mathrm{Grad}$ bewirkt bei laminarer Ablösung die Einbringung von Störungen in die abgelöste Scherschicht, während ab dem kritischen Reynoldszahlbereich wieder die anliegende Grenzschicht gestört wird.

Um bei diesem Modell wirklich die Wirkung der Wirbelerzeuger zu untersuchen und nicht die auf der Oberfläche des Zylinders stochastisch-verteilten Rauhigkeiten, wurde auch dieses Zylindermodell wie das ,Referenz"-Zylindermodell in gleicher Weise oberflächenbehandelt. Für die fotografische Aufnahme der anzufertigenden Anstrichbilder war es allerdings notwendig, die Oberfläche noch matt-schwarz zu lackieren, wodurch die mittlere Rauhigkeit etwas größer ausfällt als beim „Referenz“"-Zylindermodell, dennoch aber unterhalb von $R_{Z} \leq 5 \mu \mathrm{m}$ liegt.

\subsection{3,Aktiv“'-Zylindermodell}

Das „Aktiv“-Zylindermodell hat die umfangreichsten Aufgaben zu erfüllen. Es ist daher auch mit einer Vielzahl von Druckleitungen (Bild 2.5) und piezoelektrischen Sensoren als auch mit einem Aktuatormodul zur Umsetzung der unterschiedlichen aktiven Beeinflussungs- und Steuerungsverfahren ausgestattet (Bild 2.6). Die eingesetzte Sensorik ermöglicht stationäre wie instationäre Wanddruckmessungen durchzuführen. Stationäre Druckverteilungen geben einen guten Eindruck über das zeitlich gemittelte Verhalten der abgelösten Zylinderumströmung. Aus einer azimutalen Druckverteilung lassen sich Druckminimum, Ablösestelle, die Existenz einer Ablöseblase und der Basisdruck ermitteln. Mit einer Reihe von spannweitig verteilten stationären wie instationären Drucksensoren lässt sich das spannweitige Ablöseverhalten ermitteln. Durch Drehen des Zylinders kann darüberhinaus die spannweitige Wanddruckverteilung für mehrere Azimutwinkel zusammengetragen werden, so dass über die gesamte Oberfläche des Zylinders der Wanddruck bestimmt werden kann. Weiterhin können Korrelationen zwischen spannweitig benachbarten instationären Drucksignalen durchgeführt werden, um Aussagen über das Kohärenzverhalten der abgelösten Strömung zu erhalten.

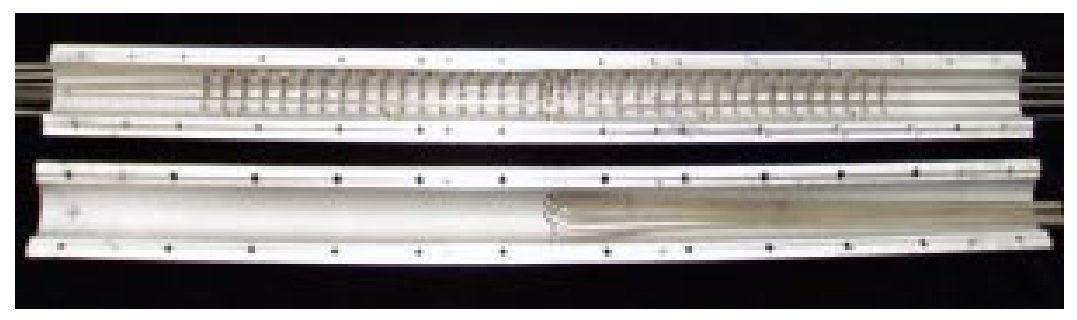

Bild 2.5 Zylinderhälften des „Aktiv“-Zylindermodells nach dem Einbau der Metallröhrchen zu den Wanddruckbohrungen für die statische Druckmessung. 
Das Aktuatormodul wurde so ausgelegt, dass sowohl die Anregung durch Ausblasen als auch durch Aufprägung periodischer Druckschwankungen prinzipiell möglich ist. Um eine hinreichende spannweitige Auflösung in der Störamplitude zu erreichen, wurde das Aktuatormodul in 36 kleine Kammern über den spannweitigen Bereich von $y_{D}=-4,4$ bis $y_{D}=+4,4$ unterteilt.
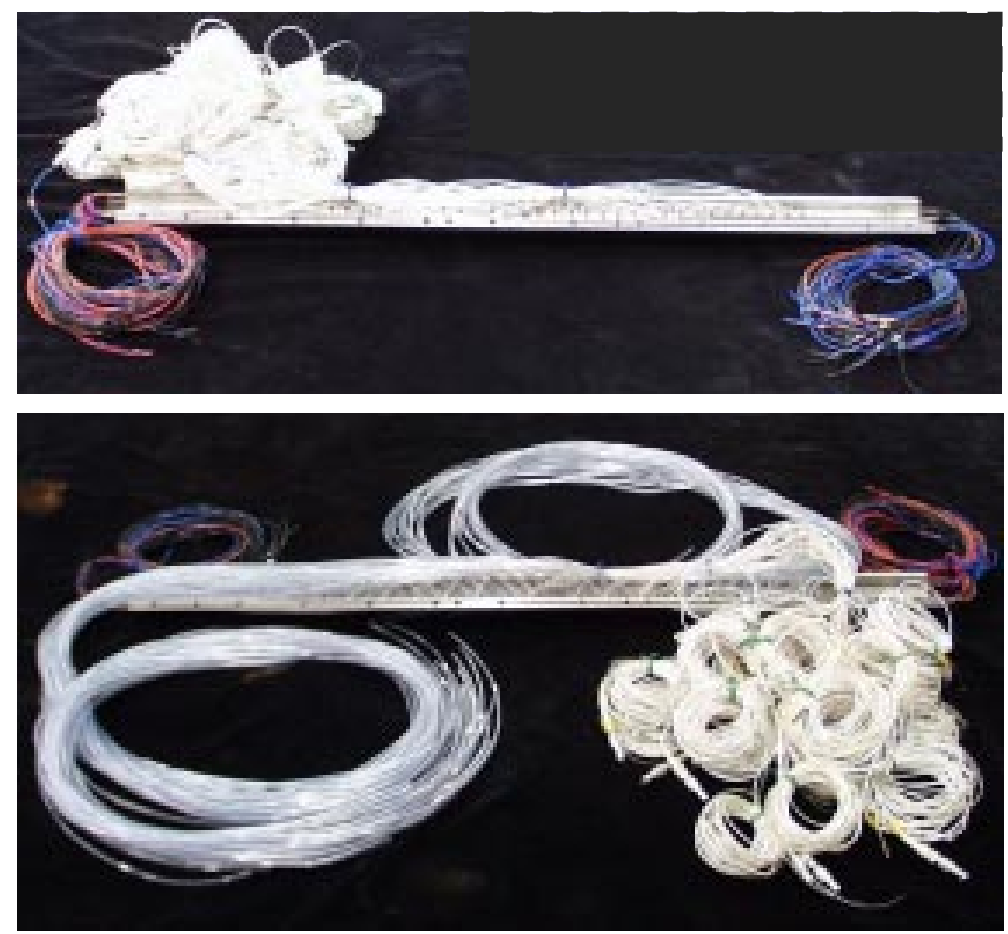

Bild 2.6 Instrumentierte Zylinderhälften des „Aktiv“-Zylindermodells vor dem Zusammenbau

Damit ergibt sich eine große Anzahl an Druckschläuchen für die stationäre Erfassung des statischen Druckes und für die Ansteuerung der Kammern des Aktuatormoduls als auch eine Vielzahl von elektrischen Leitungen für die dynamischen Drucksensoren, die aus dem Hohlraum von der Wandinnenseite oder des Mantels des Zylinders geführt werden müssen (Bild 2.6). Die Anordnung der Druckbohrungen und piezoelektrischen Sensoren in der Zylinderoberfläche musste dabei so gewählt werden, dass einerseits der störende Einfluss auf die Grenzschichtströmung so klein wie möglich gehalten wird, andererseits durch die erhaltenen Wanddrücke der Zustand und das spannweitige Verhalten der abgelösten Strömung beschrieben wird. Weiterhin ist die künstliche Störungseinbringung im unterkritischen Bereich u.a. in der Nähe der Ablösung, also bei einem Azimutwinkel von etwa $75 \mathrm{Grad}$ zu realisieren. Darüberhinaus sollte der Azimutwinkel der Störungseinbringung auch variiert werden können, womit sich der absolute Azimutwinkel der Druckbohrungen und -sensoren ebenfalls ändert und bei der Wahl der Positionen zu beachten ist. Neben Sensoren, die im Heckbereich des Zylinders eingebaut wurden, um den Basisdruck zu messen, hatte die Festlegung des Azimutwinkels einer spannweitig hochaufgelösten Drucksensorreihe auf 55 Grad stromauf der Störungseinbringung folgende Gründe:

- Die Grenzschicht wird in ihrer Stromabentwicklung vom vorderen Staupunkt bis zur Sensorreihe nur durch die in der Symmetrieebene eingebrachten Bohrungen gestört. 
- Der Druckbeiwert besitzt auch bei diesem Azimutwinkel signifikant unterschiedliche Werte in Abhängigkeit vom Strömungszustand der abgelösten Zylinderströmung, so dass die Zustände entsprechend zu den jeweiligen Reynoldszahlbereichen des laminarturbulenten Überganges anhand des Druckbeiwertes bestimmbar sind. Obwohl anhand vorliegender Druckverteilungen nach Bild 2.7 die kritischen bis überkritischen Druckbeiwerte bei 55 Grad übereinander liegen, wird sich im Experiment zeigen, dass auch hier signifikant unterschiedliche Werte zu beobachten sind (, was bereits bei der Planung des Modells vermutet wurde, da die Änderung des Ablöseverhaltens eine globale Änderung des Wanddruckfeldes nach sich zieht).

- Durch die Bohrungen der Sensorreihe wird das natürliche laminare Ablöseverhalten kaum beeinträchtigt, da die Strömung hier noch beschleunigt und gegenüber kleinen Störungen nicht instabil ist.

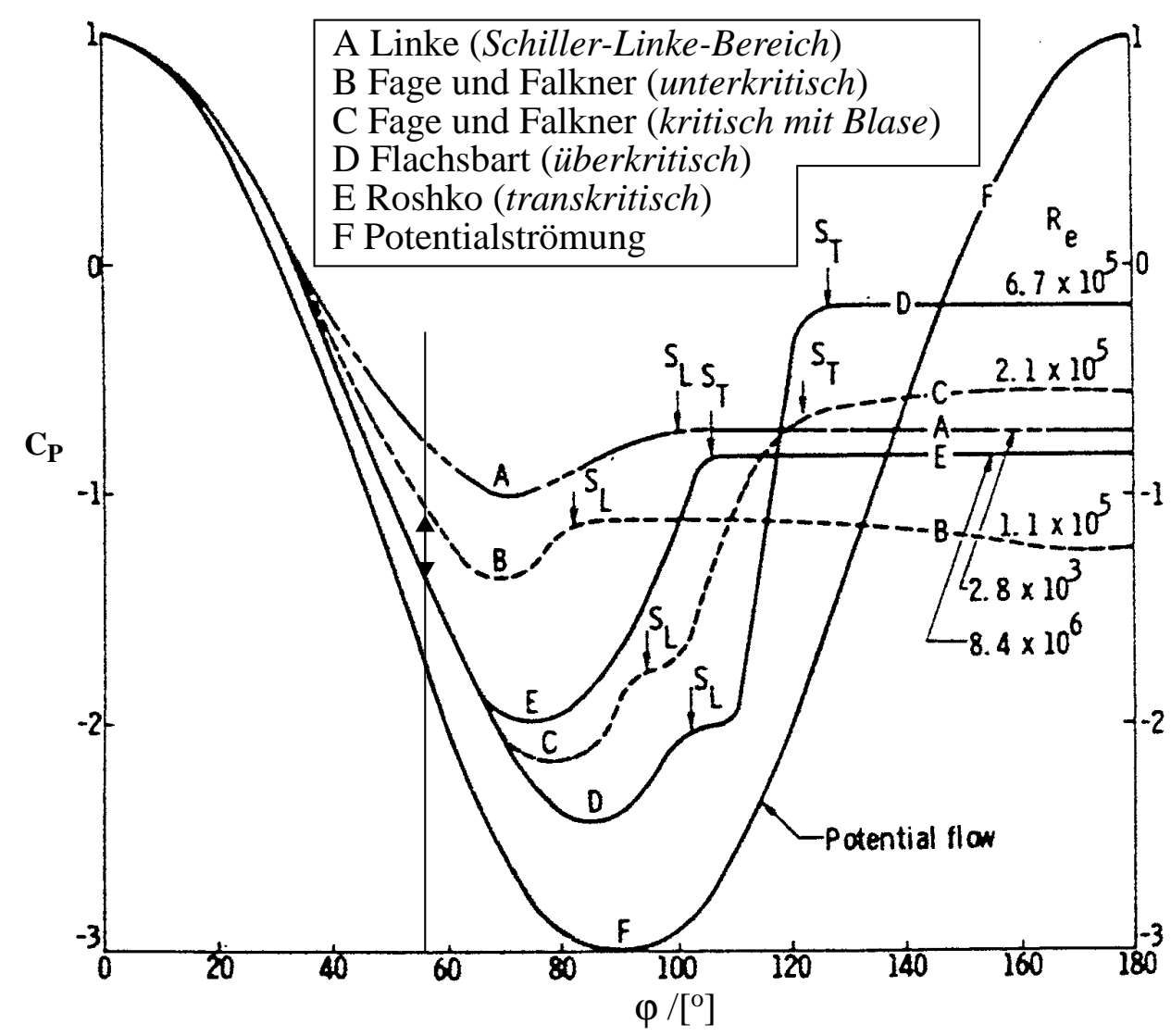

Bild 2.7 Azimutale Druckverteilungen der Zylinderströmung bei verschiedenen Reynoldszahlen im Vergleich zu der Potentialströmung (aus Morkovin 1964 [52]). Bei dem Azimutwinkel von 55 Grad ist deutlich ein Druckbeiwertunterschied für unterkritisches und kritisches bis turbulentes Ablösverhalten festzustellen.

Weiterhin wurden die stromab hintereinander liegenden Bohrungen des azimutalen Schnittes der Symmetrieebene etwas spannweitig versetzt, um bei anliegender Grenzschichtströmung eine Störungsanfachung zu vermeiden. Die Aktuatorkammer wurde im Zylindermantel in ei- 
nem Bereich von 63 bis 87 Grad untergebracht. Sie besteht aus 36 separaten Kammern, die symmetrisch zu $y_{D}=0$ angeordnet sind. Zwei Kammern haben dabei einen Mittenabstand von $\Delta y_{D}=15 \mathrm{~mm}$, während eine Kammer ein Volumen von $V_{K}=12 \times 4 \times 5 \mathrm{~mm}^{3}$ besitzt. Über den Kammern befindet sich ein dünnes, bündig abschließendes entsprechend der Zylinderoberfläche konkav-gekrümmtes Blech, welches über jeder Kammer mit 9 Bohrungen versehen ist. Die Bohrstege haben eine Länge von $l_{B}=1 \mathrm{~mm}$ und einen Durchmesser von $d_{P}=0,3 \mathrm{~mm}$. In Tabelle 2.1 sind die Anordnungen für die Druckbohrungen und Sensoren sowie für das Aktuatormodul zusammengefasst. Entsprechende schematische Darstellungen der spannweitigen Schnitte sind im Bild 2.8 gezeigt.

\begin{tabular}{|c|c|c|c|c|}
\hline Anordnung & Position & Bereich & Auflösung & Zweck \\
\hline $\begin{array}{l}\text { azimutal } \\
\text { (Oberseite) }\end{array}$ & $y_{D}=0,00$ & $\varphi_{p}=0 . .180 \mathrm{Grad}$ & $\begin{array}{l}15 \text { Grad } \\
\text { (8 Bohrungen, } \\
4 \text { Kulites })\end{array}$ & $\begin{array}{l}\text { azimutale Druck- } \\
\text { verteilung }\end{array}$ \\
\hline $\begin{array}{l}\text { azimutal } \\
\text { (Unterseite) }\end{array}$ & $\begin{array}{l}y_{D}=0,00 \\
y_{D}=+/-0,05 \\
y_{D}=+/-0,10\end{array}$ & $\varphi_{p}=0 . .-180 \mathrm{Grad}$ & $\begin{array}{l}9 \text { Grad } \\
\text { (19 Bohrungen, } \\
1 \text { Kulite) }\end{array}$ & $\begin{array}{l}\text { azimutale Druck- } \\
\text { verteilung }\end{array}$ \\
\hline spannweitig & $\varphi_{p}=55 \mathrm{Grad}$ & $y_{D}=-4,25 . .+4,25$ & $\begin{array}{l}\Delta y_{D}=0,5 \\
(18 \text { Bohrungen })\end{array}$ & $\begin{array}{l}\text { spannweitige } \\
\text { Druckverteilung }\end{array}$ \\
\hline spannweitig & $\varphi_{p}=55 \mathrm{Grad}$ & $y_{D}=-4,0 \ldots+4,0$ & $\begin{array}{l}\Delta y_{D}=0,5 \\
(17 \text { Kulites })\end{array}$ & $\begin{array}{l}\text { spannweitige } \\
\text { Druckverteilung } \\
\text { und Korrelation }\end{array}$ \\
\hline spannweitig & $\varphi_{p}=155 \mathrm{Grad}$ & $y_{D}=-4,0 \ldots+4,0$ & $\begin{array}{l}\Delta y_{D}=2,0 \\
(5 \text { Kulites })\end{array}$ & $\begin{array}{l}\text { Basisdruckmes- } \\
\text { sung; Korrelation }\end{array}$ \\
\hline spannweitig & $\varphi_{p}=180 \mathrm{Grad}$ & $y_{D}=-4,0 \ldots+4,0$ & $\begin{array}{l}\Delta y_{D}=2,0 \\
\text { (4 Bohrungen, } 1 \\
\text { Kulite) }\end{array}$ & $\begin{array}{l}\text { Basisdruckmes- } \\
\text { sung }\end{array}$ \\
\hline $\begin{array}{l}\text { Akuator- } \\
\text { modul; } \\
\text { spannweitig }\end{array}$ & $\varphi_{A}=75 \mathrm{Grad}$ & $y_{D}=-4,4 \ldots+4,4$ & $\begin{array}{l}\Delta y_{D}=0,25 \\
\text { (36 Schläuche) }\end{array}$ & 36 Kammern \\
\hline
\end{tabular}

Tabelle 2.1 Anordnung der azimutalen und spannweitigen Sensorreihen für die stationäre und instationäre Druckmessung sowie Auslegung des Akuatormoduls beim „Aktiv“Zylindermodell mit Angabe der relativen Azimutposition zum vorderen Staupunkt bei 0 Grad und der spannweitigen Positionen 

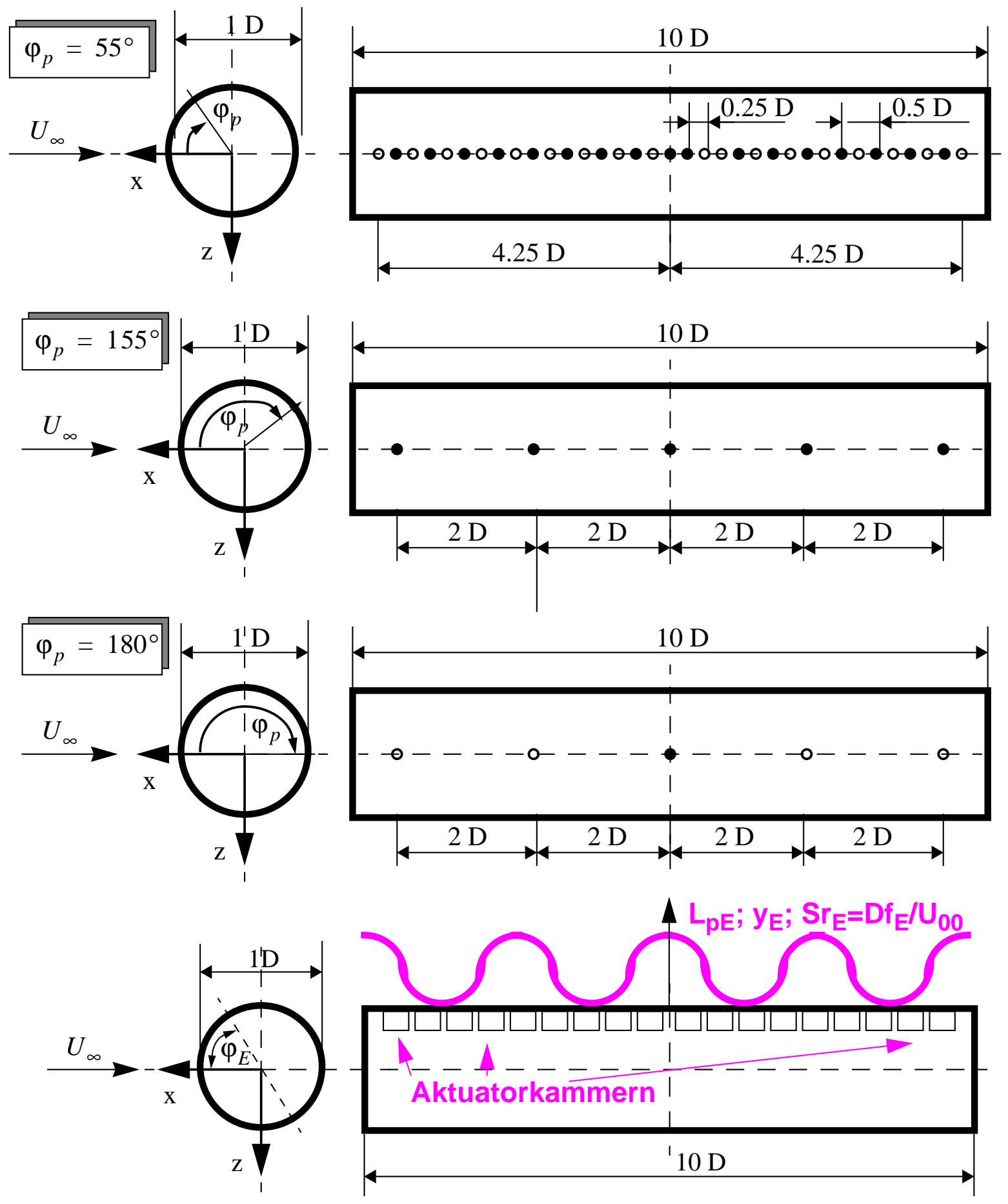

Bild 2.8 Von oben nach unten: Schematische Darstellung der Instrumentierung des „Aktiv“Zylindermodells mit verschiedenen Drucksensorreihen bei einem Azimutwinkel von $\varphi_{P}=55 \mathrm{Grad}, \varphi_{P}=155 \mathrm{Grad}$ und $\varphi_{P}=180 \mathrm{Grad}$ sowie des Aktuatormoduls bestehend aus 36 separat nutzbaren Kammern bei einem Azimutwinkel von $\varphi_{E}=75 \mathrm{Grad}$ 
Neben der passiven Störung durch Druckbohrungen ergibt sich bei Überströmung der Aktuatorkammern der Effekt einer instationären Störung aufgrund der Helmholtz-Resonanz (HR). Die Resonanzfrequenz einer Kammer mit Bohrsteg beträgt dabei nach Meyer und Guicking (1974) [49] etwa $f_{H R}=0,9 \mathrm{kHz}$ und ergibt sich aus:

$$
f_{H R}=\frac{c_{0}}{2 \pi} \sqrt{\frac{\pi}{4} \frac{d_{E}^{2}}{l_{B} V_{K}}} .
$$

Dabei stellt das federnde Volumen das Kammervolumen $V_{K}$ und die mitschwingende Masse die Luftmasse in einem Bohrsteg mit der Länge $l_{B}$ und dem Durchmesser $d_{B}$ dar. Für die Schallgeschwindigkeit wurde die unter Normbedingungen genutzt, gegeben zu $c_{O}=343 \mathrm{~m} / \mathrm{s}$. Diese hochfrequente Störung könnte die Ablösung durch Anregung der $K H$-Instabilität in der abgelösten Scherschicht beeinflussen (Bader 1997 [5], Siller et al. 1998 [83]). Anderseits lässt sich der zu erwartende Effekt nicht vermeiden, da weder das Volumen der einzelnen Aktuatorkammern noch die Länge der Bohrstege wesentlich vergrößert werden können, um eine niedrigere Frequenz zu erhalten. Weiterhin ist damit bei der künstlich einzubringenden instationären Störung der Frequenzbereich um die Helmholtz-Resonanz zu meiden. Letztendlich wird eine maximale Anregungsfrequenz von $f_{E \text {, max }}=806 \mathrm{~Hz}$ verwendet, so dass hiermit eine kontrollierte instationäre Störung in die Grenzschicht eingebracht wird.

\subsection{Messtechniken und Messverfahren}

\subsubsection{Instationäre Kraftmessungen}

Für die Messung der am Zylinder instationär angreifenden Widerstands- und Auftriebskräfte wurde ein piezoelektrisches Kraftaufnehmersystem verwendet (Schewe 1982 [74]). Das Kraftaufnehmersystem besteht dabei aus vier (Bild 2.9 rechts) piezoelektrischen Drei-Komponenten-Messelementen (Bild 2.9 links). Unter Ausnutzung des longitudinalen und transversalen piezoelektrischen Effektes werden die an diesen Scheiben in den drei Raumrichtungen angreifenden Kräfte erfasst.
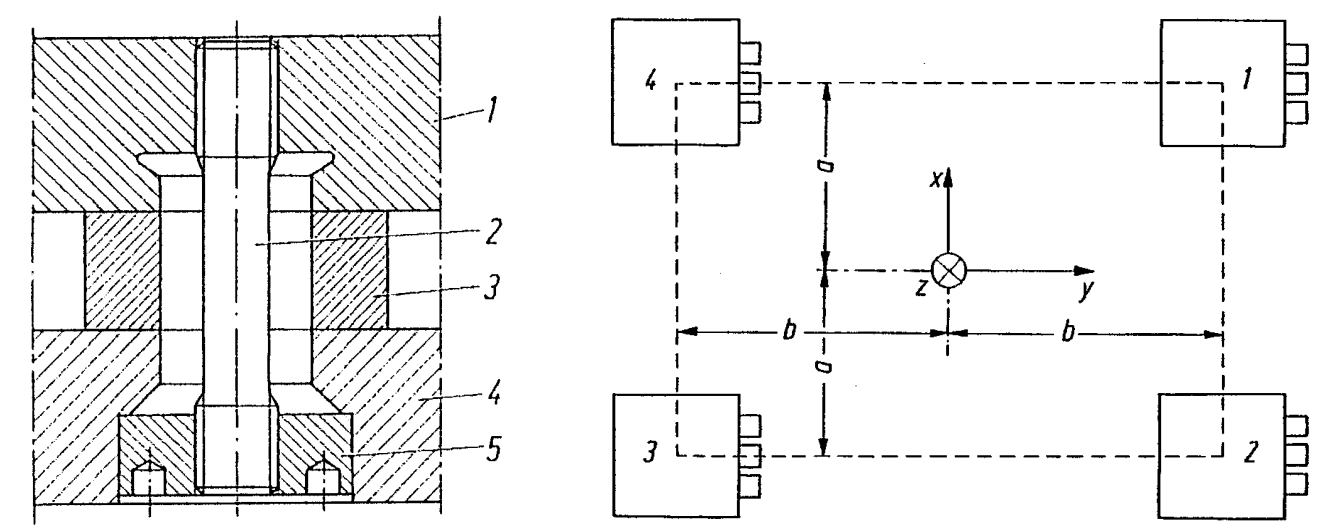

Bild 2.9 Links: Standardeinbau eines Dreikomponenten-Kraftaufnehmers (nach Kistler). 1 Kraft einleitende Deckplatte, 2 elastischer Vorspannbolzen, 3 Kraftaufnehmer, 4 Bodenplatte, 5 Ringmutter zum Vorspannen. Rechts: Vierelementiges Messystem mit je Dreikomponenten-Kraftaufnehmern (aus Tichy und Gautschi 1980 [91]) 
Proportional zu den mechanischen Kraftwirkungen entstehen in den Quarzkristalle Ladungsverschiebungen und somit Polarisationsströme, die durch Aufintegration im Ladungsverstärker in eine dazu proportionale Ausgangsspannung umgewandelt werden. Der Ladungsverstärker besitzt aufgrund der Endlichkeit der Isolation des ladungsspeichernden Kondensators $\left(R_{g}=10\right.$ TOhm) und einer Gegenkopplungskapazität $C_{g}$ auch nur endliche Zeitkonstanten $T_{g}=R_{g} C_{g}$ zwischen $10^{3}$ und $10^{6}$ Sekunden. Bei quasistatischen Messungen treten daher Leckströme auf, die eine Drift der Ausgangsspannung verursachen. Schewe (1982) [74] konnte zeigen, dass z.B. bei der Messung einer stationären Kraft von 25 N nach 17 Minuten eine Drift um 1\% festzustellen war. Durch die Erfassung der Zeit zu jedem Messpunkt, kann diese Drift durch lineare Korrektur zwischen Anfangs- und Endwert der Nullpunktspannung für jeden Messpunkt nachträglich bestimmt werden. Die Piezoelemente werden mit $100 \mathrm{kN}$ vorgespannt. Der Messbereich selbst beträgt $10 \mathrm{kN}$. Weiterhin besitzen die Piezokristalle obere Grenzfrequenzen von einigen Kilohertz und sind daher für instationäre Messungen geeignet. Darüberhinaus besitzt das System Piezowaage und Zylinder noch natürliche Eigenfrequenzen, die aber oberhalb von etwa $500 \mathrm{~Hz}$ liegen und somit zu keiner Resonanz mit der periodisch ablösende Zylinderströmung bei den verwendeten Anströmgeschwindigkeiten führen können.

\subsubsection{Instationäre Wanddruckmessungen}

Bei den instationären Druckmessungen kommen piezoresistive Wandler zum Einsatz. Der auf einer Piezomembran wirkende Druck verursacht eine Streckung bzw. Stauchung, die über eine Wheatstone'sche Brücke proportional in Spannungssignale übertragen werden. Aufgrund der geringen Empfindlichkeit von piezoresistiven Wandlern, in der Größenordnung von $3 \mu \mathrm{V} / \mathrm{Pa}$ ist das Nachschalten von Spannungsverstärkern notwendig. Je nach erwartetem Druckmessbereich in Abhängigkeit von Betriebsdruck, Anströmgeschwindigkeit und azimutaler Position der Drucksensoren wird mit einer adäquaten Einstellung der Verstärkung ein optimales SignalRausch-Verhältnis erreicht. Damit verbunden ist eine lineare Kalibrierung des Systems Sensor und Verstärker. Als Eichmaß dient eine parallel geschaltete Druckdose, die ebenfalls für die Messung der statischen Wanddrücke benutzt wird. Über einen in der Messstrecke befindenden separaten Druckbehälter wird der Kalibrierdruck künstlich vorgegeben. Wird dieser entsprechend des Messbereiches variiert, wird für jeden Sensor mit eingestellter Verstärkerstufe eine Kalibrierkurve erhalten, aus der der lineare Zusammenhang zwischen Signalspannung und Druck mittels Regression erhalten wird. Für den Messbetrieb wird über ein Mehrwegeventil der Druck im Druckbehälter wieder mit dem Druck in der Messstrecke ausgeglichen und die Referenzseite der instationären Drucksensoren mit dem Referenzdruck der Messstrecke verbunden. Ähnlich wie bei den instationären Kraftmessungen muss auch hier beachtet werden, dass die Verstärkungen über Bereichskondensatoren intern eingestellt werden. Somit ist die Messdauer mit der selben Verstärkereinstellung begrenzt, fällt hier allerdings günstiger aus und darf einige Stunden betragen.

\subsubsection{Stationäre Wanddruckmessungen}

Neben der instationären Druckmessung kommt auch die stationäre Druckmessung über Druckbohrungen zur Anwendung. Hier interessieren lediglich zeitlich gemittelte statische Drücke, die in Wandhöhe des Zylinders über Druckbohrungen und flexible Plastikschläuche an die Differenz-Drucksensoren weitergeleitet werden. Die Referenzseite misst dabei den statischen Druck 
stromauf der Messstrecke (s.a. Bild 2.11). Die Drucksensoren arbeiten wieder auf der Basis des piezoresistiven Effektes. Auch hier werden Spannungsverstärker benutzt, um die Aussteuerung des Spannungssignals für die weitere Bearbeitung der Daten zu verbessern. Da nur eine begrenzte Anzahl an diesen Differenz-Drucksensoren vorhanden ist, andererseits einige Dutzend statische Druckmessstellen für einen Messpunkt zu erfassen sind, wird die Scanivalve-Technik eingesetzt (Bild 2.10) . Sie erlaubt die Erfassung mehrerer Drücke mittels einer Druckdose. Dies ist allerdings nicht zeitgleich möglich, sondern nur zeitlich hintereinander. Die Zeitdauer für die Erfassung ergibt sich dabei aus einer Haltezeit und einer Messzeit. In der Haltezeit wird der Kanal der stationären Druckmessstelle zur Druckdose geöffnet und in der Messzeit erfolgt dann die eigentliche Messung des Druckes.
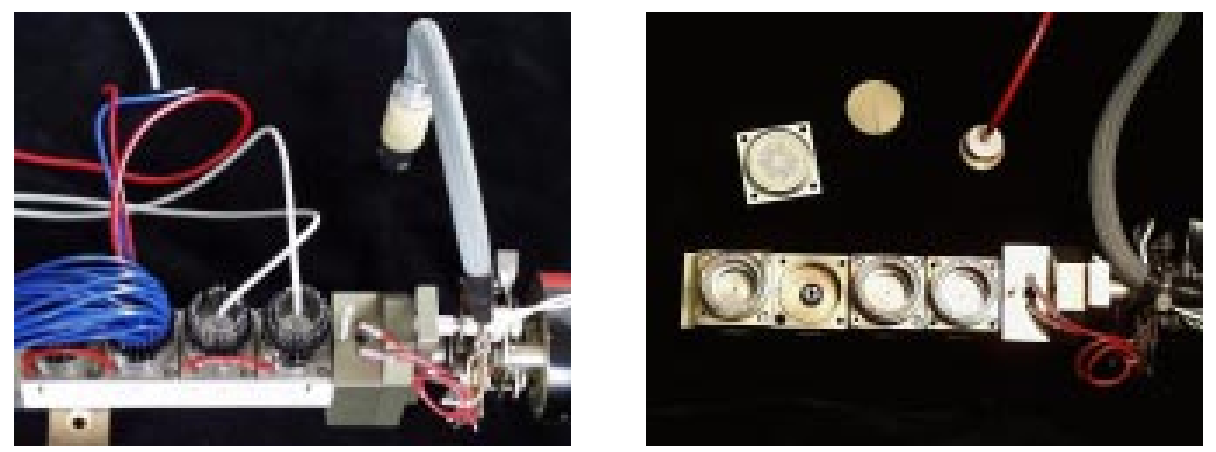

Bild 2.10 Ansicht eines Scanivalve-Systems mit vier Scanivalve-Walzen. Links mit Bestükkung eines Kopfes einer Scanivalve-Walze und rechts mit einzelnen Bestandteilen einer Scanivalve-Einheit

Insgesamt sind bis zu 16 Messstellen je Scanivalve-Walze bei maximal 4 Walzen eingerichtet worden. Mit einer Haltezeit von etwa 3 Sekunden und einer Messzeit von etwa 6 Sekunden ergibt sich damit für jede Messstelle eine Dauer von 9 Sekunden und für den Messpunkt der Messreihe etwa 150 Sekunden. Unter Beachtung der Beschränkung der Dauer einer Messreihe, wie bei den Kraftmessungen am „Referenz"“-Zylindermodell z.B. von 30 Minuten, konnten mitunter nur etwa 10 Messpunkte angefahren werden. Bei den Messungen mit dem „Aktiv“-Zylindermodell hingegen sind Messreihen mit 40 Messpunkten die Regel. Damit kann mit ausreichend hoher Auflösung in der Reynoldszahl der mögliche Geschwindigkeitsbereich von etwa $5 \mathrm{~m} / \mathrm{s}$ bis $35 \mathrm{~m} / \mathrm{s}$ in einer Messreihe abgefahren werden.

\subsubsection{Anstrichbilder}

Strömungssichtbarmachungen im Hochdruckkanal sind aufgrund der geforderten Hochdruckfestigkeit der Messtechnik mit technischen Schwierigkeiten verbunden. Da auch der optische Zugang nur schwer realisierbar ist, werden Anstrichbilder angefertigt. Sie erlauben Wandstromlinien bzw. das zeitlich gemittelte Richtungsfeld der Wandschubspannung in qualitativer Weise auf der Zylinderoberfläche sichtbar zu machen. Je nach Größe der lokalen Wirkung der Wandschubspannung wird die auf die Oberfläche eines Modells aufgetragene Suspension, einer Mischung aus Titanoxid (Titanweiß), Öl (Siemens 4503) und Petroleum, verschoben. Während das Petroleum durch die strömende Luft aus der Mischung schnell verdunstet, verbleibt die restliche Farbmischung auf der Oberfläche. Dort, wo die Wandschubspannung klein ist, sammelt sich Farbe an und kennzeichnet damit die Bildung von Ablöselinien, während Wiederanlegelinien durch ein eher quellenartig aussehendes Bild identifiziert werden können. Beeindruckende Vi- 
sualisierungen der abgelösten Strömungsstruktur sind mit Anstrichbildern möglich, wie Bippes es an einem längsangeströmten Zylinder mit kugelförmigen Kopf sehr anschaulich demonstrierte (Bippes 1990 [9]).

An einem querangeströmten Zylinder besteht die Schwierigkeit die geeignete Mischung in Abhängigkeit von der zu benutzenden Anströmgeschwindigkeit zu wählen. Einerseits wird ausreichend Farbmaterial benötigt, um die Ablösestruktur sichtbar zu machen. Andererseits kann überschüssiges Farbmaterial, was sich in der Nähe von Ablösegebieten sammeln kann, selbst bei langer Fahrzeit des Windkanals nicht antrocknen oder wird nicht ausreichend abtransportiert. Mit Abschalten des Windkanals verläuft die überschüssige Suspension auf der Zylinderoberfläche und überdeckt wesentliche Informationen des Anstrichbildes. Weiterhin kann die durch die Farbe zusätzlich aufgebrachte Oberflächenrauhigkeit das Verhalten der abgelösten Strömung verändern, so dass das bei einer Reyoldszahl erwartete Strömungsphänomen nicht beobachtet wird. Aufgrund dieser Überlegungen wurde größtenteils nur der Bereich um den vorderen Staupunkt von -45 Grad bis +45 Grad des Zylinders mit der Suspension eingestrichen. Da die Zylinderströmung zum einen in diesem azimutalen Bereich beschleunigt ist, ist sie gegenüber oberflächlichen kleinen Störungen hinreichend stabil. Zum anderen weist die Zylinderströmung in diesem azimutalen Bereich eine positive Wandschubspannung auf (Achenbach 1981 [2]), so dass ein Transport der Suspension in weiter stromabliegende Bereiche des Zylinders erfolgt. So kann später im Anstrichbild, das zeitlich gemittelte Richtungsfeld der Wandschubspannung bis zu den Ablöselinien und weiter fortsetzend ab den Wiederanlegelinien verfolgt werden.

\subsection{Datenerfassung}

Die verwendeten Messverfahren und Messtechniken liefern stationäre wie instationäre Daten, die in Echtzeit erfasst und definiert auf ein Speichermedium zur weiteren Verarbeitung und Auswertung abgelegt werden. Zwei Messdatenerfassungssysteme wurden für die Messungen am $H D G$ seitens des $D N W$ auf Workstations von Sun-Microsystems mit dem Betriebssystem UNIX zur Verfügung gestellt. Erstens DeAs (Datenerfassung und Anlagensteuerung), ein Datenerfassungssystem, welches insbesondere zeitlich gemittelte Daten aufnimmt und verwaltet und darüberhinaus auch zur Anlagensteuerung genutzt werden kann, und zweitens IDa, das Instationäre Datenerfassungssystem.

\subsubsection{Datenerfassung und Anlagensteuerung}

Die Organisation der Datenerfassung und Anlagensteuerung ist in DeAs hierarchisch und modular aufgebaut und wird in sog. Messvorhaben definiert. Dazu sind die Eigenschaften des Modells, das jeweilige Zylindermodell, und die zugehörige Instrumentierung, d.h. die Sensorik des Zylindermodells und der Messstrecke, in DeAs einzugeben. Die softwaremäßige Instrumentierung erfolgt dabei durch die Definition von Kanälen, d.h. welcher Kanal mit welchem Sensor oder Gerät verbunden ist. DeAs versteht dabei unter einem Gerät auch IDa. Durch diese Konvention ist es möglich, die instationäre Datenerfassung durch DeAs über Ethernet und TCP/IP mitzusteuern, während $I D a$ aus den erfassten Zeitreihen zeitlich gemittelte Daten an DeAs zurückliefert. Mit dem Messvorhaben ist weiterhin ein Ablaufprogramm vorzugeben, welches zur Steuerung der Messungen dient. Mit Hilfe dieses Ablaufprogramms werden Messreihen und 
eine Hierarchieebene tiefer Messpunkte definiert, die im Messbetrieb nur noch aufgerufen werden, um die zu diesem Messpunkt gehörenden Daten zu erfassen und zu speichern. Die Daten von den Sensoren oder Geräten werden dabei kanalweise über eine Datenerfassungskarte (IEEE488, RS232) eingelesen und in Dateien mit einem DeAs-spezifizierten Format in ASCIICode abgelegt. Die weitere Auswertung basiert dann auf dem Lesen dieser Datei.
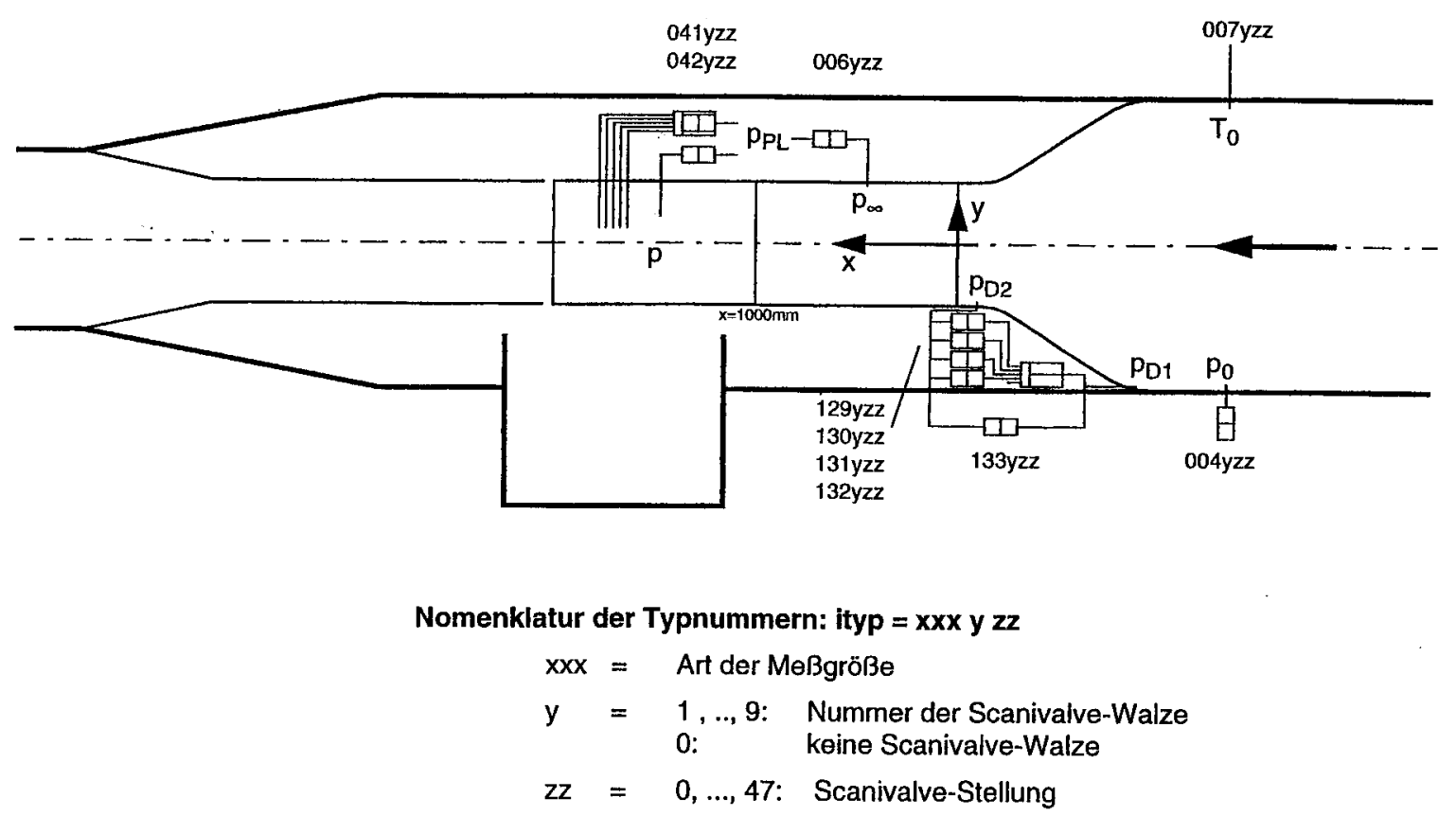

Bild 2.11 Schematische Darstellung der Sensororte am HDG und Nomenklatur der Messgrößen unter DeAs insbesondere bei Nutzung eines Scanivalve-Systems (aus Typtabellen der Auswerteschnittstelle, WK-Gö, Baumert, W; Bock, K.W.; Jacobs, M.; DNW, DLR, 1999)

Mit jedem Messpunkt werden darüberhinaus bereits eine Reihe von Messgrößen von DeAs bereitgestellt. Diese Messgrößen wie der Ruhedruck des Kanals (Betriebsdruck) $p_{0}$, die Temperatur $T_{0}$, das Düsendruckgefälle $\Delta p_{D}$ und der statische Druck $p_{\infty}$, der zwischen Düse und Messstrecke gemessen wird, dienen intern für die Steuerung der Messung. Diese gemessenen und daraus berechneten Betriebsgrößen werden dabei nicht nur jedem Messpunkt sondern auch bei der Messung des statischen Druckes $p$ mit Hilfe des Scanivalve-Systems zu jeder Scanivalve-Stellung separat bestimmt und abgespeichert (Bild 2.11). Auf diese Art und Weise können störende Einflüsse bei der langandauernden Messung mit dem Scanivalve lokalisiert werden. Insgesamt wurden fünf verschiedene Messvorhaben unterschiedlicher Komplexität eingerichtet. Insbesondere mit der Nutzung des ,Aktiv“"Zylindermodells werden je Messpunkt etwa 250 Mess- und Steuergrößen erfasst und der anschließenden Auswertung zugeführt.

\subsubsection{Instationäre Datenerfassung}

Die instationäre Datenerfassung erfolgt über modulartig aufgebaute Analog-Digital-Converter. Ein Modul kann 8 Zeitsignale mit einer Auflösung von 16 bit und einer maximalen Wandlerfrequenz von $50 \mathrm{kHz}$ einlesen, wobei die Samplingtiefe 256k Worte beträgt. Bei instationären Kraftmessungen wurde daher 1 Modul (3 Kanäle) und bei den Druckmessungen 4 Module (26 
Kanäle) genutzt. Die Eingangsempfindlichkeit wurde auf $\pm 5 \mathrm{~V}$ eingestellt. Weiterhin wurde unter IDa der Nullabgleich als auch die Zwei-Punkt-Kalibrierung genutzt. Zur Steuerung der Messung wurde ,ida_trans" benutzt. Dieses Programm dient der Erfassung hauptsächlich transienter Vorgänge und wird im ,Stand-Alone"-Betrieb realisiert, d.h. die Messungen wurden manuell oder über ein Triggersignal von DeAs gestartet.

Im Gegensatz zu DeAs musste bei IDa einem anderen Datenspeicherkonzept Rechnung getragen werden. Aufgrund der zum Teil großen Datenmengen, die bei instationären Messungen anfallen, wird bereits zu jedem Messpunkt ein Datenfile angelegt, welches im Binärformat strukturiert erstellt wird. Die Struktur eines IDa-Meßpunkt-Files besteht aus einem Kopf, der neben der Beschreibung allgemeiner Parameter wie Anzahl Kanäle, Kanaltiefe, etc. auch Kalibrationsdaten und Nullpunktsdaten enthält und natürlich die eigentlichen Messdaten von jedem Kanal. Dieses Fileformat kann einfach in das CDF-Format konvertiert werden. In der Programmiersprache $\mathrm{C} / \mathrm{C}++$ geschriebene eigene Programme ermöglichen dann eine weitere Auswertung der Daten.

\subsection{Experimenteller Versuchsaufbau}

Der experimentelle Versuchsaufbau ist modulartig aufgebaut. Die in den Zylindermodellen implementierten oder mit ihnen verknüpften Sensoren wurden in drei unabhängig voneinander aufgebauten Messketten strukturiert. In Bild 2.12 sind die einzelnen Messketten für die statische Druckmessung mit Scanivalve, die instationäre Druckmessung und die instationäre Kraftmessung dargestellt. Sie werden mit Nutzung der verschiedenen Zylindermodelle entsprechend kombiniert:

- „Referenz“"Zylindermodell mit den Messketten für dynamischen Kraftmessung und statischen Wanddruckmessung,

- „Passiv“-Zylindermodell mit der Messkette für dynamischen Kraftmessung,

- ,Aktiv“-Zylindermodell mit den Messketten für statische und dynamische Druckmessungen.

Darüberhinaus gestattet der Aufbau des Aktuatormoduls beide Beeinflussungsverfahren, das Absaugen und die akustische Anregung, ohne größere Umbauten einzusetzen (Bild 2.13). Beim kontinuierlichen Absaugen wird über die Aktuatorkammer, die mit einem Luftdruck von $p_{0}=$ 3,5 bar beaufschlagt ist, einfach Luft aus dem Kanal an die Atmosphäre abgelassen. Der dabei eintretende Druckverlust im Kanal ist vernachlässigbar. Um etwa gleiche Messbedingungen sicherzustellen, wird aber nach jeder Messreihe der Kanal wieder auf den ursprünglichen Kanaldruck aufgepumpt. Beim Absaugen (Bild 2.13 links) wird der Volumenstrom $Q$ durch die Aktuatorkammern über ein Regelventil am Durchflussmesser (mit Schwebekörper) eingestellt. Dabei wird der Volumenstrom soweit erhöht bis eine signifikante Änderung im Basisdruck zu beobachten ist und im folgenden für alle Messreihen mit kontinuierlicher Absaugung konstant gehalten. Die mittlere Absaugegeschwindigkeit $w_{Q}$ über $N$ Kammern je $n=9$ Bohrstegen mit einem Durchmesser $d_{E}=0,4 \mathrm{~mm}$ ist durch die Kontinuitätsgleichung bestimmt zu:

$$
w_{Q}=\frac{\rho_{A t m}}{\rho_{0}} \frac{Q_{E}}{N n \frac{\pi}{4} d_{E}^{2}} .
$$




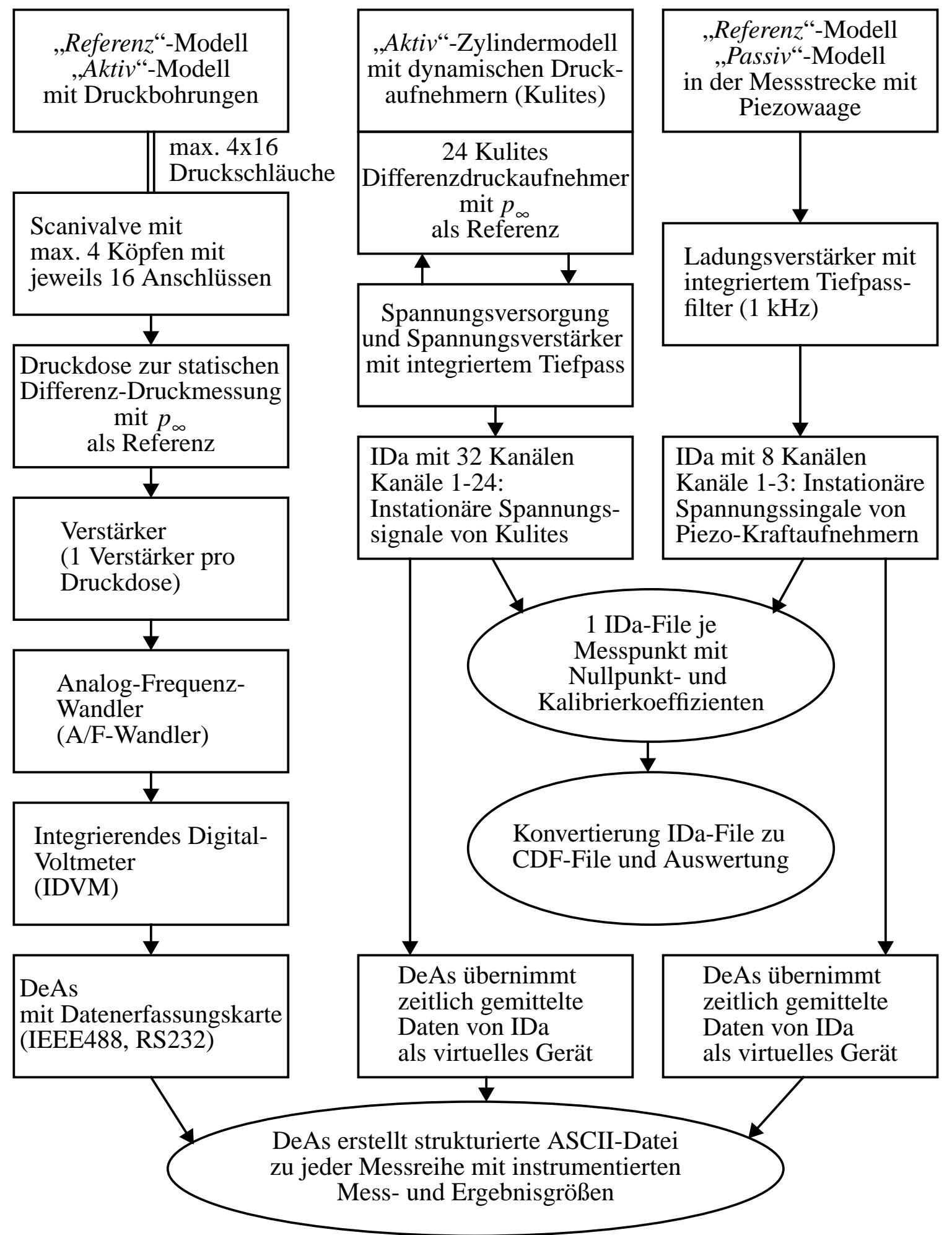

Bild 2.12 Blockdiagramm der drei Messketten und des Signalflusses für die statische Druckmessung (links), für die dynamische Druckmessung mit Kulites (Mitte) und die dynamische Kraftmessung mit der Piezowaage (rechts) 


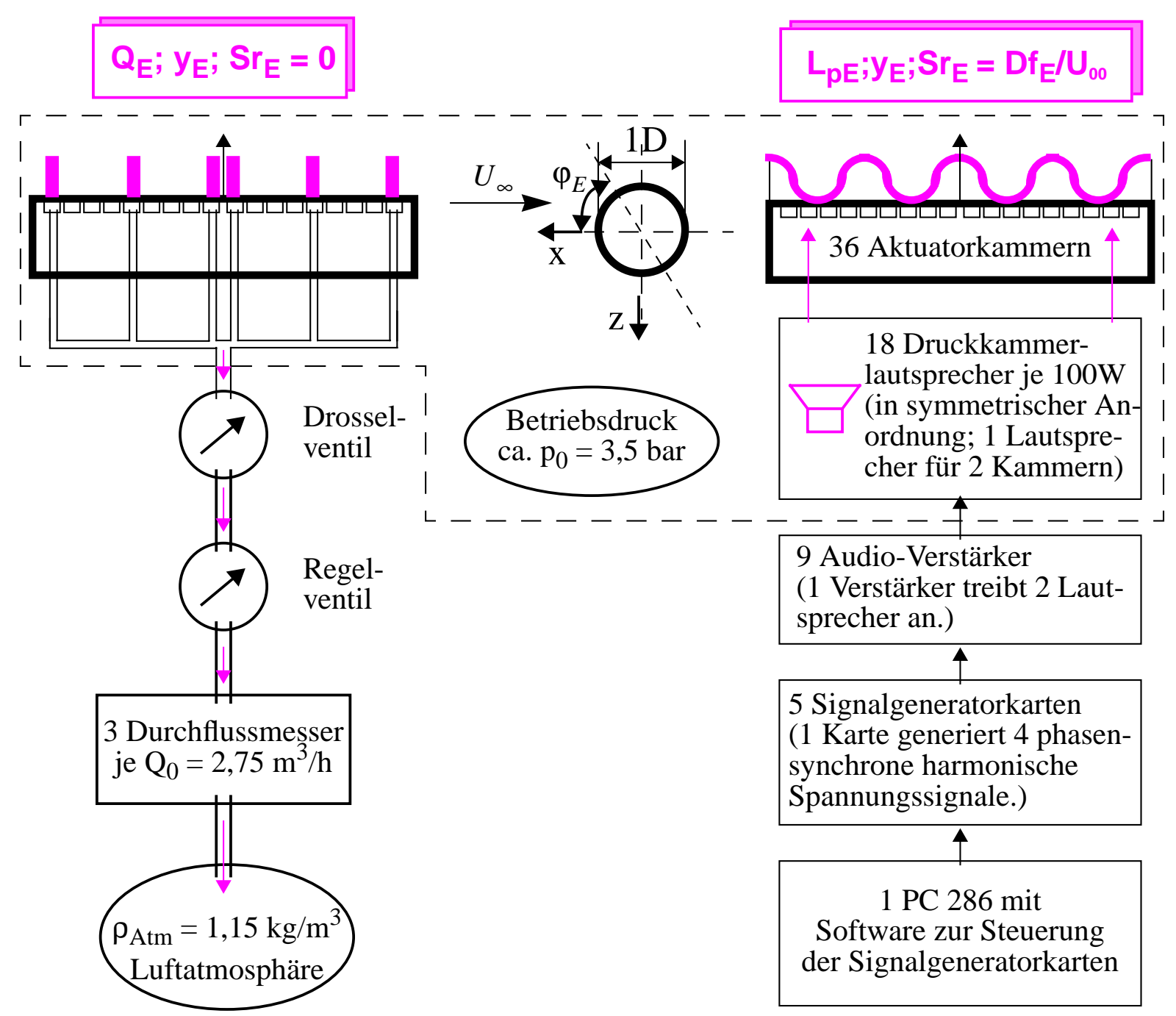

Bild 2.13 Schematische Darstellung der Versuchanordnung für das Absaugen (links) und für die akustische Anregung mittels Druckkammerlautsprecher (rechts)

Bei der akustischen Beeinflussung (Bild 2.13 rechts) werden für die Störsignalerzeugung softwaregesteuerte Signalgeneratorkarten verwendet, die harmonische Spannungssignale auf die Druckkammerlautsprecher mit je $100 \mathrm{~W}$ geben. Durch zwischengeschaltete Leistungsverstärker werden die jeweiligen Signalstärken eingestellt und auch Leistungsunterschiede zwischen den einzelnen Lautsprechern ausgeglichen. Die Signalform wird durch eine Reihe von diskreten Spannungswerten festgelegt, die über eine Programmschleife immer wieder durchlaufen werden. Eine Signalform wird hierbei diskret mit etwa 1000 Stützpunkten erzeugt, was eine ausreichende Auflösung darstellt. Mit fünf Signalgeneratorkarten werden die 18 Lautsprecher über ein Triggersignal phasensynchron angesteuert. Diese erzeugen dann periodische Druckschwankungen, die über Druckschläuche und über die Aktuatorkammern bis zur Zylinderoberfläche sich ausbreiten. In Wandnähe des Zylinders entsteht somit eine periodische Freistrahl- und Senkenströmung, wobei die durch den Freistrahl bewirkte Störgeschwindigkeit vom Betrag wesentlich größer ist als die der Senkenströmung. Mit der Laser-Doppler-Anemometrie konnte dieses 
asymmetrische Verhalten gezeigt werden (Siller et al. 1998 [83]). Die damit verbundene Schallschnelle $w_{P}$ in den Bohrstegen der Aktuatorkammern kann über die Bestimmung des Schalldruckpegels abgeschätzt werden. Der Schalldruckpegel $L_{p E}$ wird dazu in einem Abstand von a $=1 \mathrm{~mm}$ über der Oberfläche des Zylinders mit Hilfe eines Kondensatormikrofons mit dahintergeschaltetem Verstärker gemessen. Wie beim Absaugen wurde auch der Schalldruckpegel solange variiert bis eine deutliche Basisdruckverbesserung festgestellt wurde und im folgenden konstant gehalten. Lediglich für den Vergleich der Wirkung der akustischen Anregung wurden unterschiedliche Schalldruckpegel realisiert: $L_{p E}=92 \mathrm{~dB}\left(p_{\text {eff }}=0,8 \mathrm{~Pa}\right), L_{p E}=86 \mathrm{~dB}\left(p_{\text {eff }}=0,4\right.$ $\mathrm{Pa})$ und $L_{p E}=80 \mathrm{~dB}\left(p_{\text {eff }}=0,2 \mathrm{~Pa}\right)$. Wegen der komplizierten Geometrie der Aktuatorkammern und des geringen Durchmessers der Bohrstege $d_{E}$ gegenüber der Sensorfläche des Mikrofons wurde für die Bestimmung der Schallschnelle nach Mechel 1989 [46] (S. 277ff) sowohl das Modell des ,atmenden Zylinders“ als auch der ,atmenden Kugel“ für die Beschreibung der mitschwingenden Masse herangezogen. Damit wird für den tatsächlichen Wert der Schallschnelle als Störamplitude in Abhängigkeit von der Anregefrequenz $f_{E}$ eine untere $w_{P, Z y l}$ und eine obere Schranke $w_{P, \text { Kugel }}$ angegeben. Die Herleitung des Zusammenhangs zwischen Schallschnelle und Schalldruck für den ,atmenden Zylinder“ ist in Raguse (1998) [64] zu finden. Analog ist sie für die ,atmende Kugel“ durchzuführen. Das Ergebnis lautet:

$$
w_{P, Z y l}=\frac{1}{d_{E} \ln \left(2 \pi f_{E} / c_{0} a\right)} \frac{p_{e f f}}{\sqrt{2} f_{E} \rho_{0}}, w_{P, \text { Kugel }}=\frac{32 a}{d_{E}^{2}} \frac{p_{e f f}}{\sqrt{2} \pi f_{E} \rho_{0}}
$$

und liefert folgendes $\underline{\text { Bild 2.14 }}$

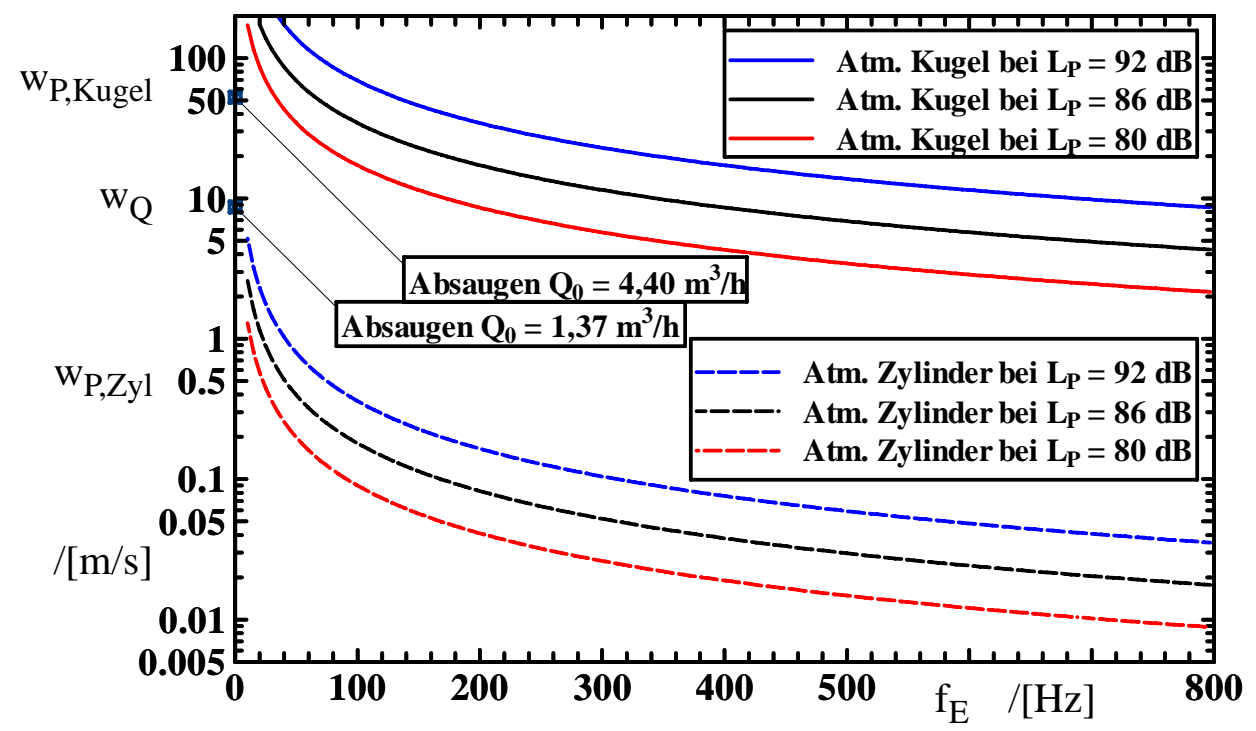

Bild 2.14 Schallschnellen für den ,,atmenden Zylinder“ und für die ,,atmende Kugel“ bei drei verschiedenen Schalldrücken nach Gleichung $\{2.3\}$ und die mittlere Absaugegeschwindigkeit für zwei Volumenströme bei $N=12$ Kammern nach Gleichung $\{2.2\}$

Zum Vergleich zur instationären Störung sind auch die mittleren Absaugegeschwindigkeiten an der Wand für zwei typische Volumenströme für $N=12$ Kammern nach Gleichung $\{2.2\}$ mit in das Bild bei $f_{E}=0 \mathrm{~Hz}$ eingetragen. Insbesondere bei der spannweitig periodischen Absaugung wurden diese Volumenströme verwendet. Obwohl die Störamplituden für die stationäre und instationäre Störung damit die gleiche Größenordnung annehmen könnten und wie später gesehen 
wird, auch vergleichbare Widerstandsreduktionen erreicht werden, ist ein direkter Vergleich in der Effektivität beider Beeinflussungsverfahren nicht möglich.

\subsection{Experimentelle Bestimmung physikalischer Größen}

In die Bestimmung von Kraft- und Druckbeiwerten aus den Kraft- und Druckmessungen gehen weitere grundlegende Größen wie Dichte und Anströmgeschwindigkeit ein. Darüberhinaus wird z.B. die Reynoldszahl benötigt, um formale Zusammenhänge darstellen zu können. Da die Messungen bei variablen Betriebsbedingungen stattfinden, verändern sich die Stoffeigenschaften der strömenden Luft im Kanal. Mit der Genauigkeit von Dichte, Viskosität und Kompressibilität in Abhängigkeit vom Luftdruck und Temperatur ist somit auch die Exaktheit der Ergebnissgrößen beschränkt.

\section{Dichte, Anströmgeschwindigkeit und Reynoldszahl}

Dichte, Anströmgeschwindigkeit und Reynoldszahl sind für die Durchführung einer Messung grundlegende Größen. Sie basieren auf der Messung von Temperatur, Luftdruck und Staudruck. Während sich die Meßsensoren für die Temperatur und den Druck in der Vorkammer des Windkanals befinden, wird der Staudruck über das Düsendruckgefälle ermittelt (siehe Bild 2.11). Der Referenzdruck, der später für die Berechnung der statischen Druckbeiwerte benötigt wird, wird mit einem weiteren Differenzdrucksensorpaar bestimmt. Dies ist notwendig, da das Messstrekkenmodul aus dem Messstreckenbereich des Kanals leicht herausnehmbar und wieder einbaubar sein muss.

Der Temperatursensor ist ein Widerstandsthermometer auf der Basis von Nickel. Nickel zeichnet sich durch einen linearen Zusammenhang zwischen Temperatur und ohmschen Widerstand in dem Temperaturbereich von $-250{ }^{\circ} \mathrm{C}$ bis $+60{ }^{\circ} \mathrm{C}$ und im Vergleich zu z.B. Kupfer, Gold oder Silber durch einen großen Temperaturkoeffizienten aus.

Der Luftdruck wird mit einem Hochpräzisions-Drucksensor gemessen. Dieser erlaubt Drücke bis 103.4 bar (1500 psi) in dem relevanten Temperaturbereich sehr genau mit Hilfe einer VierFolien-Dehnungsmeßstreifenanordnung, die in Form einer Wheatstone'schen Brücke geschaltet ist, zu messen.

Für die Messung des Staudruckes hingegen werden Differenzdruckdosen, die piezoresisitiv arbeiten, eingesetzt. Um die Genauigkeit in der Bestimmung des Staudruckes in Abhängigkeit von dem Druckbereich zu erhöhen, stehen vier Druckdosen mit den Messbereichen 75 mbar, 150 mbar, 500 mbar und 1000 mbar zur Verfügung. Bei der Messung eines Messpunktes unter DeAs werden alle vier Staudrücke simultan erfasst und der Wert mit der besten Aussteuerung für die weitere Auswertung der Messdaten verwendet. Bei der Berechnung des Staudruckes geht auch der Kanalfaktor ein, der für die geschlossene Messstrecke $k=1,0404$ beträgt.

Die Dichte der Luft $\rho$ ergibt sich aus der Zustandsgleichung, wobei der Kompressionsmodul $G$ in Abhängigkeit von Luftdruck und Lufttemperatur sowie molare Gaskonstante $R_{m}$ der Luft miteingeht (VDI Wärmeatlas. VDI-Verlag, Düsseldorf, 4. Aufl., 1974).

$$
\rho_{0}\left(p_{0}, T_{0}\right)=\frac{p_{0}}{R_{m} T_{0} G\left(p_{0}, T_{0}\right)} .
$$

Die Anströmgeschwindigkeit $U_{\infty}$ folgert aus der Bernoulli Gleichung und lässt sich aus dem Düsendruckgefälle $\Delta p_{D}$ gemäß 


$$
U_{\infty}=\sqrt{\frac{2 k \Delta p_{D}}{\rho_{0}}}
$$

berechnen.

Um die Reynoldszahl $R e_{D}$ zu bestimmen, bedarf es der dynamischen Zähigkeit $\mu$, die ebenfalls eine Funktion des Druckes und der Temperatur darstellt. Ebenfalls mit Daten aus dem o.g. VDI Wärmeatlas (1974) ergibt sich eine Interpolationsformel zur Bestimmung von $\mu\left(p_{0}, T_{0}\right)$. Die Reynoldszahl lässt sich somit berechnen zu:

$$
R e_{D}=\frac{D U_{\infty} \rho_{0}\left(p_{0}, T_{0}\right)}{\mu\left(p_{0}, T_{0}\right)} .
$$

Mit der Bestimmung des Staudruckes und der Anströmgeschwindigkeit ist es nunmehr möglich die Beiwerte aus den Kraft- und Druckgrößen als auch die Strouhalzahl der Wirbelablösefrequenz zu berechnen.

\section{Bestimmung der Kraftbeiwerte}

Die Messung der Kräfte erfolgt mit einer vierelementigen Piezowaage, wobei für die Bestimmung des Widerstandsbeiwertes nur zwei Komponenten und für den Auftriebsbeiwert nur eine Komponente erfasst werden müssen. Ladungsverstärker mit integrierten Tiefpassfiltern stehen am Ende der Messkette und liefern drei unkalibrierte Spannungssignale für die weitere Auswertung. Die Signale werden dabei von $I D a$ mit einer bestimmten Taktfrequenz von zumeist $f_{S}=2$ $\mathrm{kHz}$ und mit einer Länge des Signals von zumeist $N_{S, O}=16384$ Samples aufgenommen, wobei die Tiefpassfrequenz $f_{g}=1 \mathrm{kHz}$ beträgt, um den Aliasing-Effekt zu vermeiden (Bendat und Piersol 1971 [8]). Nach einer Nullpunkts- und Driftkorrektur der Spannungssignale werden diese in Krafteinheiten umgerechnet, wobei der Kalibrierfaktor 20 N/V beträgt. Die Gesamtwiderstandskraft $W$ ergibt sich gemäß dem Aufbau aus den Kraftkomponenten $F_{x 1}$ und $F_{x 2}$, während die Auftriebskraft $A$ sich aus der verbleibenden Komponente $F_{z}$ erhalten wird.

Mit der Querschnittsfläche des Zylindermodells $L^{x} D$ folgt der Widerstandsbeiwert respektive der Auftriebsbeiwert zu:

$$
\begin{gathered}
C_{W}=\frac{1}{D L q_{\infty}} \frac{1}{N_{S, 0}}\left\{\sum_{i}^{N_{S, 0}} F_{x, 1}(i)+\sum_{i}^{N_{S, 0}} F_{x, 2}(i)\right\} \\
C_{A}=\frac{1}{D L q_{\infty}} \frac{1}{N_{S, 0}}\left\{\sum_{i}^{N_{S, 0}} F_{z}(i)\right\}
\end{gathered}
$$

und der Effektivwert des Auftriebs zu:

$$
c_{A, \text { eff }}=\frac{1}{D L q_{\infty}} \sqrt{\frac{1}{N_{S, 0}}\left(\sum_{i}^{N_{S, 0}} F_{z}(i)^{2}-\frac{1}{N_{S, 0}}\left(\sum_{i}^{N_{S, 0}} F_{z}(i)\right)^{2}\right)}
$$

Weiterhin ist nicht nur der Effektivwert der Auftriebsschwankungen von Interesse, sondern auch dessen spektrale Verteilung. Das Amplitudenspektrum wird dabei über die Strouhalzahl dargestellt $\mathrm{zu}$ :

$$
\left|c_{A}(S t)\right|=\frac{\Delta f}{D L q_{\infty}} \sqrt{\left|\widehat{F}_{z}(f \rightarrow S r)\right|}
$$


mit

$$
f(i) \rightarrow \operatorname{Sr}(i)=i \frac{f_{s} D}{N_{s} U_{\infty}} .
$$

Um eine größere statistische Sicherheit zu erreichen, wurde über mehrere Spektren aus einer Zeitreihe je nach geforderter Auflösung in der Strouhalzahl gemittelt. Typische Samplebreiten betragen $N_{S}=256, N_{S}=512$ und $N_{S}=1024$. Bei einer Überlappung von $50 \%$ wurde somit über $M=127, M=63$ und $M=31$ Spektren gemittelt.

\section{Bestimmung der Druckbeiwerte}

Bei der Bestimmung der Druckbeiwerte müssen zwei Messketten verfolgt werden - die Messung mit den instationären Drucksensoren und die Messung mit den Differenzdruckdosen. Letztere Sensoren liefern ein Spannungssignal, welches über einen Verstärker und AnalogFrequenz-Wandler auf ein Frequenz-Digital-Voltmeter gegeben und mit DeAs aufgezeichnet wird. Intern werden die Kalibrierfaktoren von Differenzdruckdose und Verstärker auf das Spannungssignal angewendet, so dass mit der rechnerischen Verknüpfung als Ergebnis die statischen Druckbeiwerte zur Verfügung stehen. Bei der Messkette der dynamischen Messung des Wanddruckes wird zur Erfassung der Spannungssignale IDa benutzt, so dass die Bestimmung der Beiwerte analog zur Bestimmung der Kraftbeiwerte vorgenommen werden. Mit der vor jeder Messreihe durchgeführten Kalibrierung der Kulites sind die Kalibrierfaktoren für jeden Messpunkt bestimmt, so dass nach der Dekodierung der binären Zeitreihe zu einem Sapnnungssignal ein kalibriertes, nullpunktkorrigiertes Drucksignal für jeden Sensor vorliegt. Auch hier wird zumeist eine Samplingfrequenz von $f_{S}=2 \mathrm{kHz}$ und eine Sampleanzahl von $N_{S, 0}=16384$ verwendet. Damit ergeben sich die Druckbeiwerte zu:

$$
\begin{gathered}
C_{P}=\frac{1}{q_{\infty}}\left\{\frac{1}{N_{S, 0}} \sum_{i}^{N_{S, 0}}\left(p(i)-p_{\infty}\right)\right\} \\
c_{p, \text { eff }}=\frac{1}{q_{\infty}} \sqrt{\frac{1}{N_{S, 0}}\left(\sum_{i}^{N_{S, 0}} p(i)^{2}-\frac{1}{N_{S, 0}}\left(\sum_{i}^{N_{S, 0}} p(i)\right)^{2}\right)} \\
\left|c_{p}(S r)\right|=\frac{\Delta f}{q_{\infty}} \sqrt{|\widehat{p}(f \rightarrow S r)|}
\end{gathered}
$$

Um auch hier eine größere statistische Sicherheit in der spektralen Amplitude der Druckschwankungen zu erreichen, wurde ebenfalls über $M=127, M=63$ oder $M=31$ Spektren je nach geforderter Auflösung in der Strouhalzahl gemittelt.

\section{Bestimmung der Strouhalzahl der Wirbelablösefrequenz}

Aus den Amplitudenspektren der Auftriebs- und Druckschwankungen lässt sich die Strouhalzahl bestimmen. Sie wird identifiziert durch den maximalen Wert im Spektrum. Insbesondere im unterkritischen, überkritischen und transkritischen Reynoldszahlbereich zeichnet sich die spektrale Verteilung um die Wirbelablösefrequenz durch Schmalbandigkeit aus. 


\section{Ergebnisse zur natürlich abgelösten Zylinderströmung}

Der querangeströmte Kreiszylinder ist ein bereits häufig experimentell untersuchter Körper. Trotzdem wird bis heute der Instabilitätsmechanismus des laminar-turbulenten Überganges der abgelösten Zylindergrenzschicht nicht vollständig verstanden. Mit dem Messen von Kräften und Wanddrücken als auch mit der Anfertigung von Anstrichbildern werden neue Erkenntnisse zum instationären und dreidimensionalen Verhalten der Ablösung vorgestellt. Dabei kommt für die Beschreibung der Dynamik das „Konzept der dominanten Wirbelablösefrequenz" von Morkovin (1964) [52] zur Anwendung. Für die Identifizierung von komplexen Ablösestrukturen ist die topologische Denkweise maßgebend (Dallmann 1983 [19]). So wird gezeigt, dass Änderungen der topologischen Ablösestruktur mit Änderungen im instationären Verhalten verknüpft sind. Andererseits muss sich erst im unterkritschen Bereich eine bestimmte topologische Struktur ausbilden, damit die drastischen Änderungen im zeitlich gemittelten wie im instationären Ablöseverhalten der Zylindergrenzschicht beim laminar-turbulenten Übergang vonstatten gehen können.

\subsection{Verhalten von Beiwerten und Strouhalzahl}

Das Verhalten des Widerstands- und Auftriebsbeiweiwertes sowie des Effektivwertes der Auftriebsschwankungen und der Strouhalzahl der Wirbelablösefrequenz in Abhängigkeit von der Reynoldszahl ist für die Beschreibung der abgelösten Zylinderströmung charakteristisch. Abweichungen in diesen Werten sind zumeist auf unterschiedliche Experimentierbedingungen, wie Turbulenzgrad der Anströmung, die Oberflächenrauhigkeit des Zylindermodells oder die Modellabmaße wie Längen-zu-Durchmesser-Verhältnis, Versperrungsverhältnis sowie der Typ der verwendeten Messstrecke, zurückzuführen. Daher werden die Messergebnisse mit denen von Schewe (1983) [75] verglichen. Das verwendete „Referenz"-Zylindermodell stimmt in den geometrischen Eigenschaften mit denen des Modells von Schewe überein und die Messungen wurden ebenfalls im $H D G$ mit dem selben piezoelektrischen Kraftaufnehmersystem durchgeführt. In Bild 3.1 sind der Widerstandsbeiwert, die Strouhalzahl der dominanten Wirbelablösefrequenz und der Effektivbeiwert der Auftriebsschwankungen in Abhängigkeit von der Reynoldszahl für einen Bereich von $R e_{D}=0,05^{\times} 10^{6}$ bis $R e_{D}=5,0^{\times} 10^{6}$ zusammen mit den Ergebnissen von Schewe (1983) [75] aufgetragen, wobei die eigenen Messungen erst bei einer Reynoldszahl von $R e_{D}=0,08^{\times} 10^{6}$ beginnen. Auf das Verhalten des Auftriebsbeiwertes wird später noch detaillierter eingegangen (siehe 3.3 Kritischer Übergangsbereich).

Die Messergebnisse stimmen gut überein. Die Reynoldszahlbereiche und der Verlauf der Werte in diesen Bereichen kommen gut zur Deckung. Insbesondere das Verhalten der Beiwete bei den

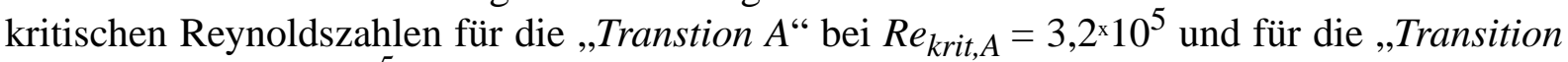
$B^{\text {“ bei }} \operatorname{Re}_{k r i t, B}=3,5^{\times} 10^{5}$ sind mit denen von Schewe identisch. Abweichungen sind lediglich für Reynoldszahlen im oberen Übergangsbereich von etwa $R e_{D}=1,0^{\times} 10^{6}$ bis etwa $R e_{D}=3,0^{\times} 10^{6}$ festzustellen. Diese könnte durch mikroskalige Makulaturen in der Oberfläche oder durch kleine Partikel, die auf der Oberfläche des Zylinders zurückgeblieben sind, begründet sein (Schewe 2000 [79]). 

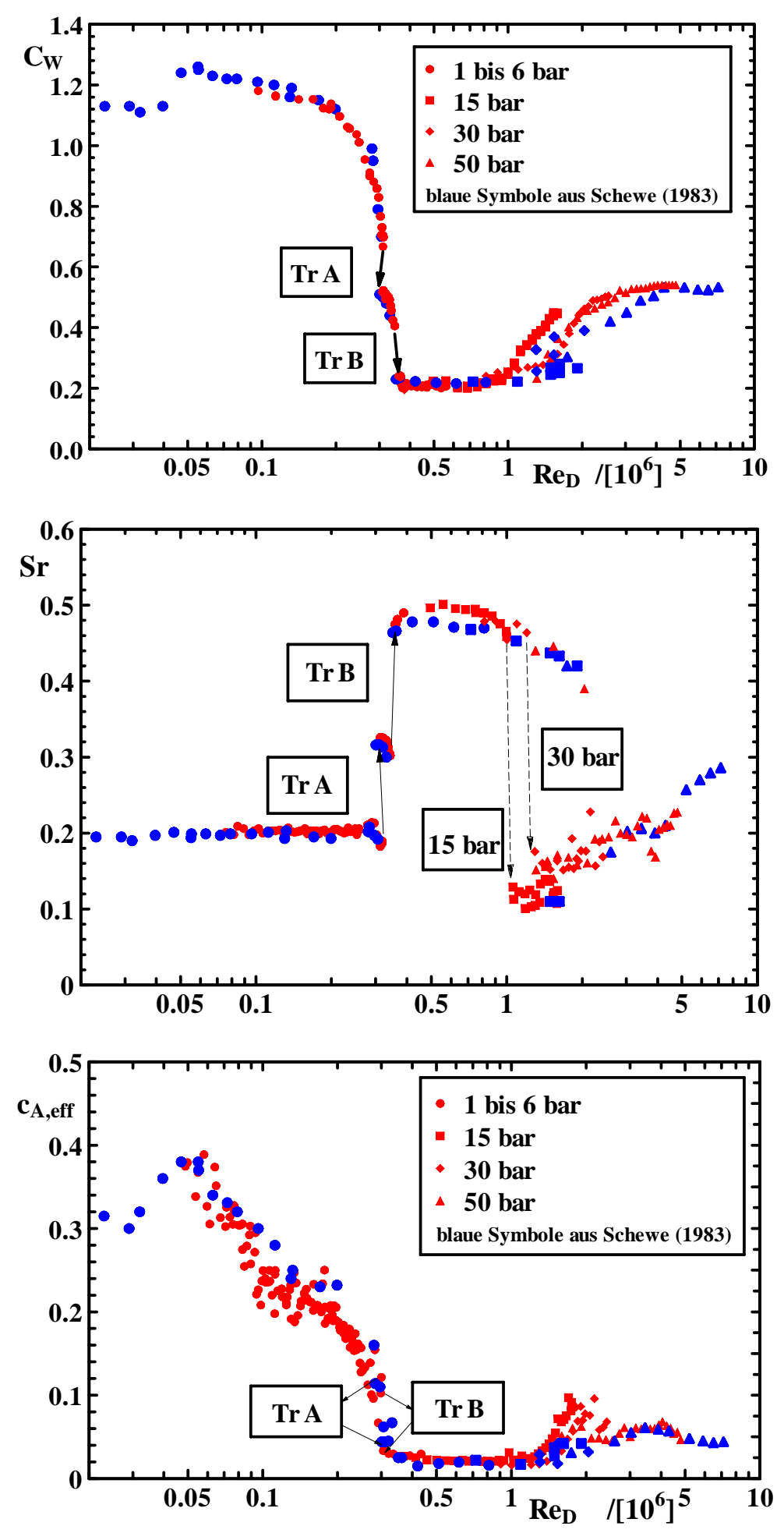

Bild 3.1 Von oben nach unten: Widerstandsbeiwert, Strouhalzahl und Effektivwert der Auftriebsschwankungen in Abhängigkeit von der Reynoldszahl bei der Transition der ablösenden Zylindergrenzschicht im Vergleich mit Ergebnissen von Schewe (1983) [75] 
Der überkritische Strömungszustand mit beidseitig ausgebildeten transitionellen Ablöseblasen ist sensitiv gegenüber kleinsten oberflächlichen Störungen. Diese können das Verschwinden bzw. die Störung der Ausbildung der transitionellen Ablöseblase und so ein vorzeitiges turbulentes Ablösen der Grenzschicht bewirken. Damit verbunden ist ein Wiederansteigen des Widerstandsbeiwertes. Mit der Änderung des zeitlich periodischen Ablöseverhaltens zu Anfang des oberen Übergangsbereiches ist ein Anwachsen der Auftriebsbeiwertschwankungen zu beobachten. In der Auftragung über der Reynoldszahl ist dies an der Ausbildung eines lokalen Maximums bei etwa $R e_{D}=2,0^{\times} 10^{6}$ gut zu erkennen. In diesem Reynoldszahlereich beobachtete Schewe ein ambivalentes Verhalten im Widerstandsbeiwert, d.h. das Auftreten mehrerer Zustände zu einer Reynoldszahl. Dieses Phänomen konnte hier innerhalb einer Messreihe nicht gefunden werden. In Bild 3.1 sind für den Reynoldszahlbereich von $R e_{D}=1,0^{\times} 10^{6}$ bis $R e_{D}=$ $2,2^{\times} 10^{6}$ der Widerstandsbeiwertverlauf aus zwei unterschiedlichen Messreihen dargestellt, die auf dieses ambivalente Verhalten des Widerstandsbeiwertes hindeuten. Es kann aber auch sein, dass der Unterschied in diesen beiden Messreihen wieder auf eine geringe Änderung in der Oberflächenrauhigkeitsverteilung zurückgeführt werden muss. Eine mögliche Erklärung für dieses Verhalten wird in 3.4 Übergang vom überkritischen zum transkritischen Bereich gegeben.

Trotz dieser Abweichungen in dem oberen Übergangsbereich repräsentieren die Beiwerte und die Strouhalzahl im Vergleich zu den Ergbnissen von Schewe eine Zylinderströmung mit gleichem Widerstands- und Auftriebsverhalten.

\section{2 Änderung des Ablöseverhaltens im unterkritischen Bereich}

Der Widerstandsbeiwert im unterkritischen Reynoldszahlbereich zeichnet sich über den gesamten Bereich durch einen nahezu konstanten Wert bei etwa $C_{W}=1,1$ aus. Diese Konstanz beruht auf dem Transitionsverhalten der abgelösten Scherschichtströmung. Während im Schiller-Linke-Bereich der Transitionsort in der abgelösten Scherschicht mit zunehmender Reynoldszahl immer weiter stromauf wandert und der Widerstandsbeiwert kontinuierlich zunimmt, verbleibt der Transitionsort in den abgelösten Scherschichten für den unterkritischen Reynoldszahlbereich in einem gewissen Abstand vom Zylinder. Die Länge des Rezirkulationsgebietes beträgt dabei nur etwa einen Zylinderdurchmesser (Schiller und Linke 1933 [80]). Andererseits erfolgt mit Anfang des kritischen Bereiches - ab etwa $\operatorname{Re}_{D}=2,0 \times 10^{5}$ - ein kontinuierlicher Abfall des Widerstandsbeiwertes, während die Strouhalzahl mit $S r=0,2$ zunächst konstant bleibt. Wie ist dieses Verhalten zu erklären?

Bekanntermaßen löst die laminare Grenzschicht bei einem Azimutwinkel von etwa 75 Grad beidseitig periodisch ab, wobei auf der Ober- und Unterseite des Zylinders eine Ablöselinie beobachtet wird. Erst Anstrichbilder von Schewe und Dallmann (1987) [20] zeigten das Auftreten von zwei Ablöselinien bei 73 Grad und 90 Grad auf jeder Seite des Zylinders bei einer Reynoldszahl von $\operatorname{Re}_{D}=1,85^{\times} 10^{5}$. Damit bewiesen sie, dass die Änderung der topologischen Ablösestruktur von einer Ablöselinie (Bild 3.2 a) zu einer mit Ablöseblase (Bild 3.2 c, d) über diese Ablösestruktur (Bild 3.2 b) erfolgt. Diese Ablösestruktur von zwei Ablöselinien mit einer dazwischenliegenden Anlegelinie kann auch als eine doppelte Ablöseblase aufgefasst werden und wird im folgenden so bezeichnet. Das bedeutet phänomenologisch, dass diese Ablösestruktur in der Lage ist, den kontinuierlichen Abfall im Widerstandsbeiwert mitzutragen ohne, dass sich das periodische Ablöseverhalten ändert. Um den Übergang zwischen diesen beiden Ablösestrukturen aufzuzeigen, wurden Anstrichbilder am „Passiv“-Zylindermodell und spannweitige 
Druckverteilungsmessungen am ,Aktiv“-Zylindermodell im unterkritischen Bereich durchgeführt. Beim Fotografieren der Anstrichbilder konnten optische Reflexionen nicht vermieden werden. Sie stören teilweise die Interpretation der hier abgebildeten Ansichten der Anstrichbilder. Daher sind im Anhang A.3 Anstrichbilder der natürlich abgelösten Zylinderströmung weitere Ansichten zu jedem Anstrichbild dargestellt.

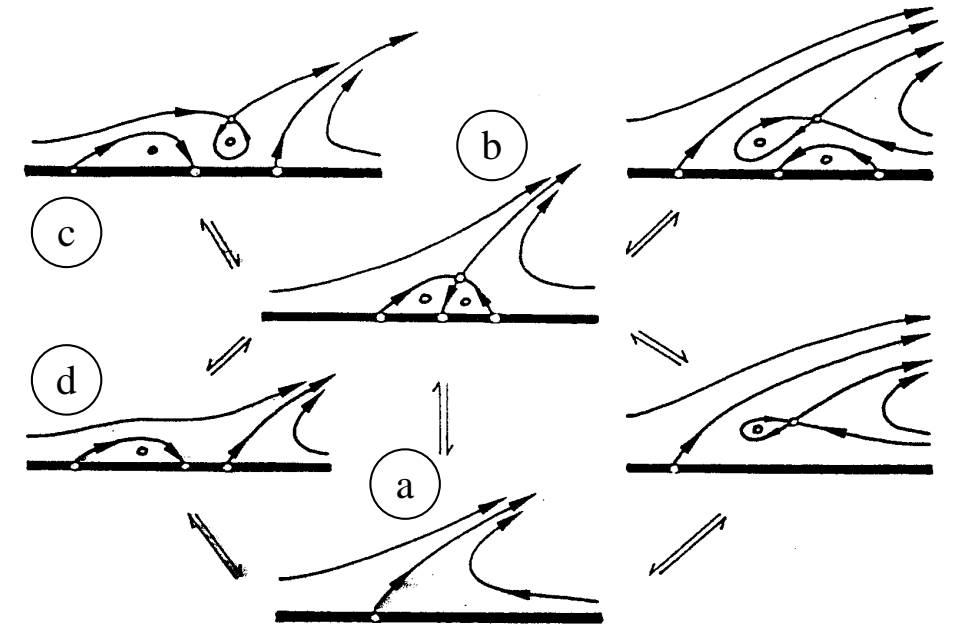

Bild 3.2 Schematische Darstellung der Topologie möglicher zeitlich gemittelter Ablösestrukturen der Zylinderströmung aus Dallmann und Schewe (1987) [20]. Der Übergang von der Ablösestruktur mit einer Ablöselinie (a) zu der mit Ablöseblase (c, d) vollzieht sich über den Zustand einer Ablösestruktur von zwei Ablöselinien mit einer dazwischenliegenden Anlegelinie (b), also einer doppelten Ablöseblase.

\section{Anstrichbilder}

Tatsächlich wird aus einer Reihe von Anstrichbildern im unterkritischen Reynoldszahlbereich diese Änderung in der Ablösestruktur sichtbar. Bei der Reynoldszahl von $\operatorname{Re}_{D}=1,2^{\times} 10^{5}$ tritt jeweils nur eine Ablöselinie auf jeder Seite des Zylinders bei etwa 76 Grad auf (Bild 3.3). Im Anstrichbild zu der Reynoldszahl von $R e_{D}=2,0^{\times} 10^{5}$ sind deutlich die beiden Ablöselinien auf jeder Seite des Zylinders bei etwa 76 Grad und 92 Grad zu erkennen (Bild 3.4). Die dazwischenliegende Anlegelinie kann nur vermutet werden. Das Vorhandensein von Farbe zwischen den beiden Ablöselinien deutet aber darauf hin, dass Fluidmaterial hier wieder auf die Oberfläche trifft. Darüberhinaus könnte vermutet werden, dass die Anlegelinie sich nicht stationär verhält, sondern mit der periodischen Ablösung der Grenzschicht sich zwischen den beiden Ablöselinien mehr oder weniger stark hin und her bewegt.

Der Übergang von der einen zu der anderen topologischen Struktur vollzieht sich auf beiden Seiten des Zylinders nicht bei der selben Reynoldszahl. Insbesondere im Anstrichbild bei $R e_{D}$ $=1,7 \times 10^{5}$ (Bild 3.5) ist ein topologisch asymmetrischer Zustand zu beobachten. Während auf der Oberseite sich lediglich eine Ablöselinie bei etwa 78 Grad befindet, sind auf der Unterseite zwei Ablöselinien bei etwa 76 Grad und 92 Grad zu erkennen. Die zweite Ablöselinie ist dabei nicht über der gesamten Spannweite durchgängig. Bei etwa $\left|y_{D}\right|=3,0$ ist jeweils ein Einbruch in der Ablöselinie festzustellen. Weiterhin zeigt das Anstrichbild Farbvorträge auf beiden Seiten bei den Spannweiten von etwa $\left|y_{D}\right|=1,0$. Weitere Anstrichbilder zu den Reynoldszahlen $R e_{D}=$ $1,5^{\times} 10^{5}$ und $\operatorname{Re}_{D}=1,9^{\times} 10^{5}$ sind im Anhang A.3 Anstrichbilder der natürlich abgelösten Zylinderströmung dargestellt. Sie untermauern, dass der Übergang in der Ablösestruktur nicht ohne 
Zwischenzustände erreicht wird. Bemerkenswert hierbei ist, dass der Auftrieb auch mit der Ausbildung der asymmetrischen Ablösestrukturen im zeitlichen Mittel verschwindet.
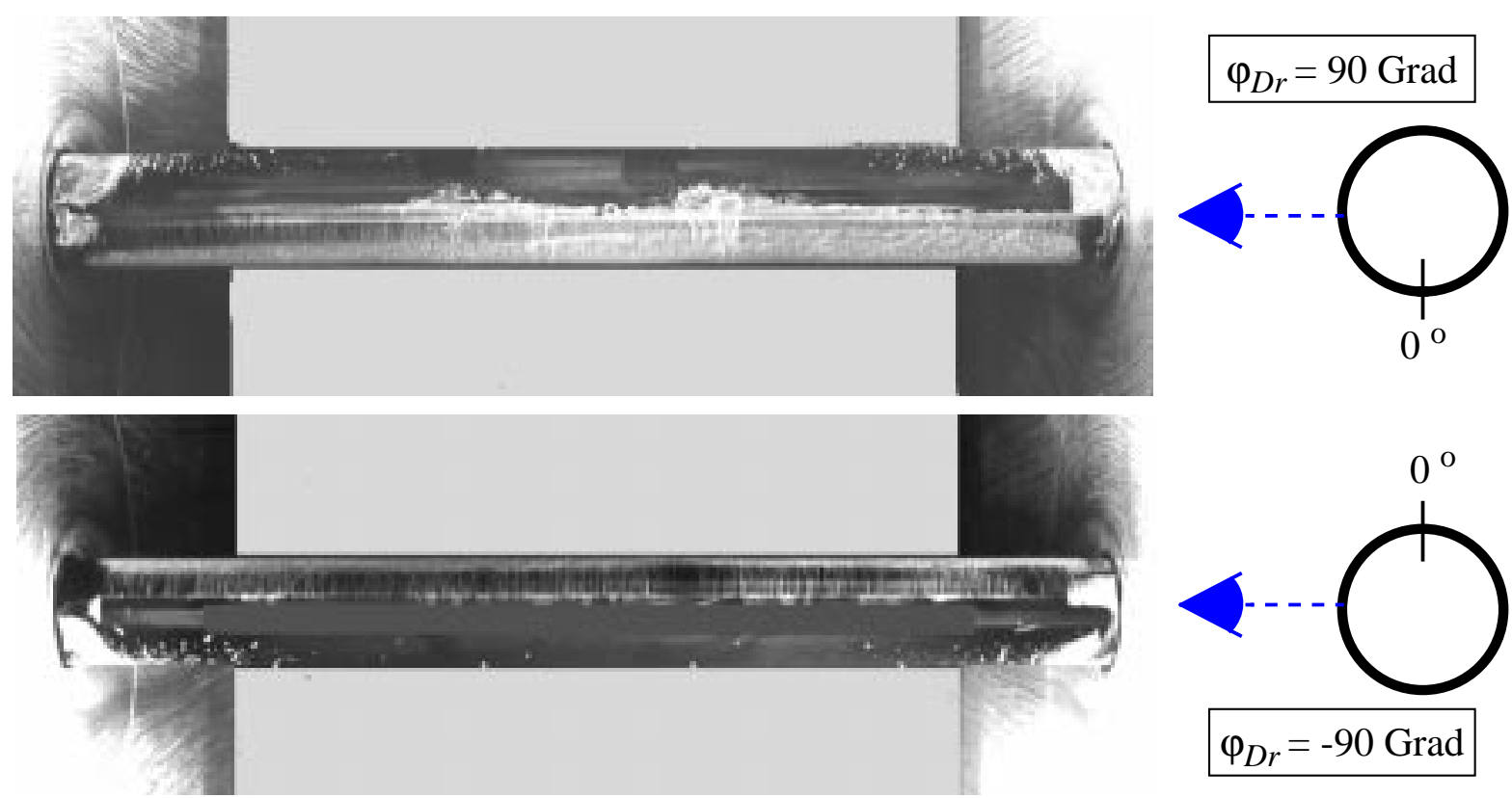

Bild 3.3 Anstrichbild bei $R e_{D}=1,2^{\times} 10^{5}$ mit einer Draufsicht unter einem Winkel von +90 Grad (oben) bzw. -90 Grad (unten) zeigt jeweils eine Ablöselinie auf der Ober- und Unterseite des Zylinders bei einem Azimutwinkel von etwa +76 bzw. -76 Grad (s.a. Bild A2.1).

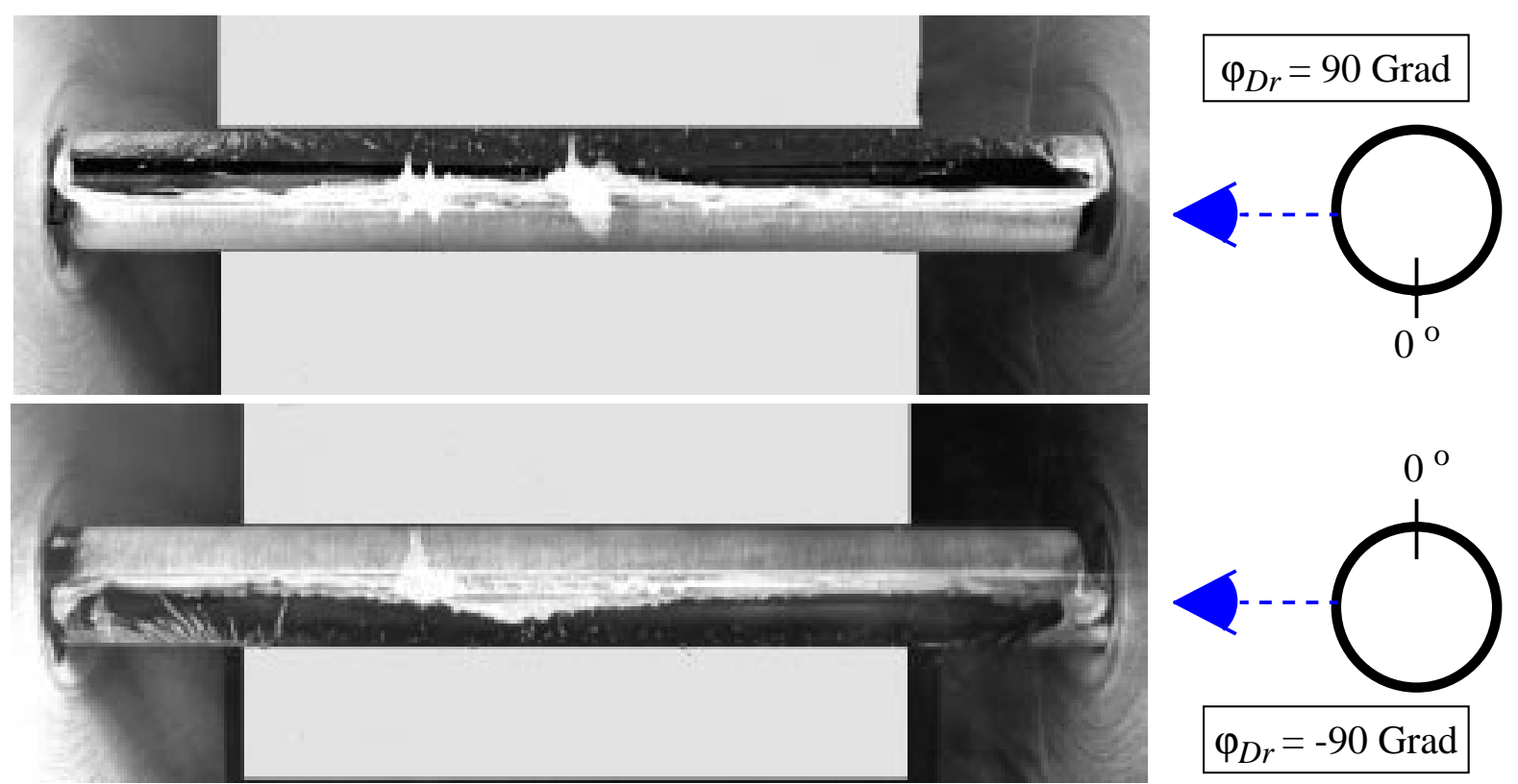

Bild 3.4 Anstrichbild bei $R e_{D}=2,0^{\times} 10^{5}$ mit einer Draufsicht unter einem Winkel von +90 Grad (oben) und -90 Grad (unten) zeigen die topologisch neue Ablösestruktur der doppelten Ablöseblase zwischen etwa 76 und 92 Grad jeweils auf Ober- und Unterseite des Zylinders (s.a. Bild A2.5 und Bild A2.4). 


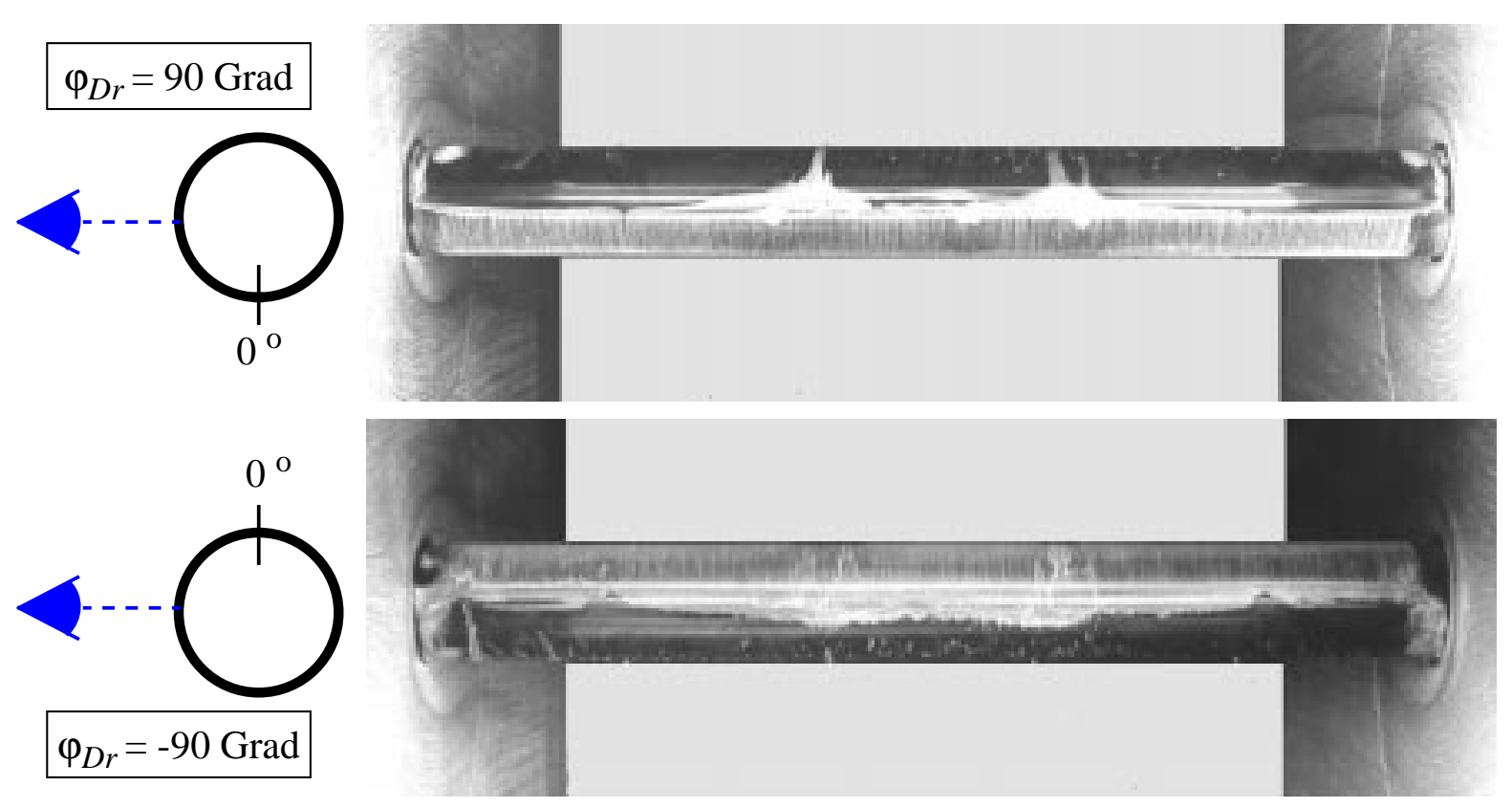

Bild 3.5 Anstrichbild bei $R e_{D}=1,7 \times 10^{5}$ mit einer Draufsicht unter einem Winkel von +90 Grad (oben) und -90 Grad (unten). Auf der Oberseite ist nur eine Ablöselinie bei etwa 78 Grad und auf der Unterseite die doppelte Ablöseblase zwischen 76 und 92 Grad zu erkennen (s.a. Bild A2.3).

Die bei den Anstrichbildern erhaltenen Widerstandsbeiwerte entsprechen sehr gut den Widerstandsbeiwerten der natürlich abgelösten Zylinderströmung mit glatter Oberfläche, so dass die eingangs postulierte Verschiebung zu kleineren Reynoldszahlen für den unterkritischen Reynoldszahlbereich hier nicht zutrifft (s.a. Bild 3.14). Scheinbar spielt die durch die Farbe zusätzlich aufgebrachte Oberflächenrauhigkeit keine wesentliche Rolle für diesen Übergang in der Ablösestruktur.

Angemerkt sei noch, dass Anstrichbilder, die an einem Zylindermodell ohne Endscheiben im 1Meter-Windkanal Göttingen (1MG) mit offener Messstrecke angefertigt wurden, ebenfalls diesen Übergang in der Ablösestruktur im unterkritischen Bereich zeigen. Darüberhinaus sind die Ablöselinien bei beiden Ablösestrukturen deutlich ausgebildet und weisen keinerlei Störungen längs der Spannweite auf.

\section{Spannweitige Druckverteilungen}

Neben der Anfertigung von Anstrichbildern wurden auch spannweitige Wanddruckverteilungen im unterkritischen Reynoldszahlbereich gemessen. Durch Drehen des Zylinders wurde das Druckfeld in einem Azimutwinkelbereich zwischen 60 Grad und 125 Grad erhalten. In Bild 3.6 sind somit für die Reynoldszahlen $R e_{D}=1,5^{\times} 10^{5}, R e_{D}=1,8^{\times} 10^{5}$ und $R e_{D}=2,1^{\times} 10^{5}$ der statische Druckbeiwert über Spannweite und Azimutwinkel aufgetragen.

Der Druckbeiwert erreicht bei diesen Reynoldszahlen sein Minimum bei etwa $65 \mathrm{Grad}$. Die spannweitige Druckverteilung in diesem Minimum weist eine Periodizität von etwa $y_{L}=2 D$ auf. Bei der Reynoldszahl $R e_{D}=2,1 \times 10^{5}$ ist diese spannweitig periodische Modulation des Druckbeiwertes im Minimum nicht mehr ausgeprägt, wenn auch die Druckverteilung spann- 
weitig nicht konstant ist. Andere Autoren haben diese spannweitig periodische Modulation des Druckes im Minimum der azimutalen Druckverteilung ebenfalls gefunden. So beobachten Higuchi et al. (1989) [34] für einen Zylinder mit einem Streckungsverhältnis von $L / D=8$ in dem Azimutwinkelbereich von $60 \mathrm{Grad}$ bis $80 \mathrm{Grad}$ eine spannweitig periodische Ausbildung des Druckminimums bei einer Reynoldszahl von $R e_{D}=1,95^{\times} 10^{5}$. Auch hier beträgt die spannweitige Wellenlänge etwa $y_{L}=2 D$. Obwohl das Durchmesserverhältnis der Druckbohrungen $\left(d_{P}=\right.$ $0,8 \mathrm{~mm})$ zum Zylinder $(D=4,42 \mathrm{~cm})$ recht groß ist, scheinen diese das laminare Ablöseverhalten nicht gestört zu haben.

Die wesentlich kleinere spannweitig periodische Modulation von $y_{L}=0,5 \mathrm{D}$ beruht auf der Ungenauigkeit der Kalibrierung der Kulites im Vergleich zu der Differenzdruckdose, die den statischen Wanddruck misst. Die spannweitige Länge entspricht gerade dem Abstand zweier dynamischer Sensoren.
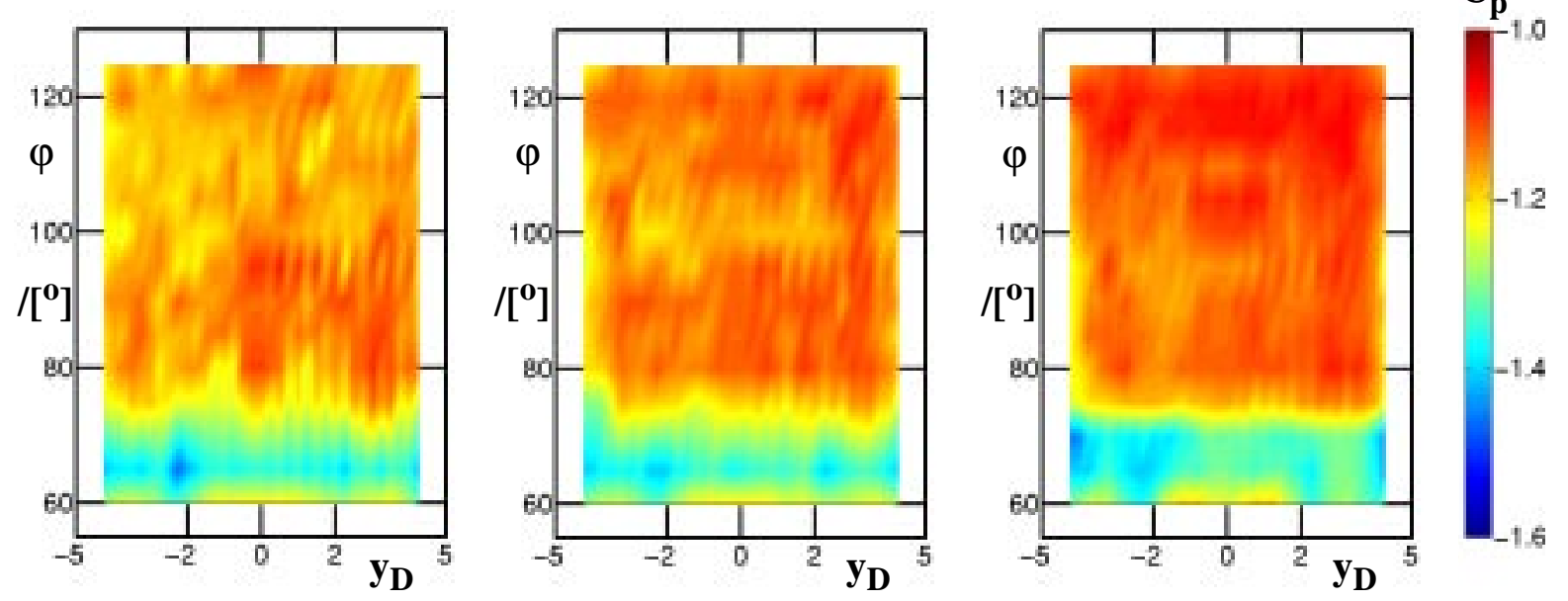

Bild 3.6 Spannweitige Druckverteilung bei $\operatorname{Re}_{D}=1,5^{\times} 10^{5}$ (links), $\operatorname{Re}_{D}=1,8^{\times} 10^{5}$ (Mitte) und $R e_{D}=2,1 \times 10^{5}$ (rechts) für einen azimutalen Bereich von $60 \mathrm{Grad}$ bis $125 \mathrm{Grad}$.

Einen Hinweis auf die Änderung der topologischen Ablösestrukur ist aus den Darstellungen des Druckbeiwertes nicht ablesbar. Vermutlich ist die azimutale Auflösung mit 5 Grad zu grob, um diese erfassen zu können. (Hinsichtlich der spannweitigen Auflösung ist zu beachten, dass die kleinskalige Modulation in den spannweitigen Druckverteilungen auf ein unterschiedliches Kalibriermaß des dynamischen und der statischen Druckmessung zurückzuführen ist und nicht ein Effekt von kleinskaligen Längswirbeln ist.) Andererseits weisen die spannweitigen Druckverteilungen für Reynoldszahlen der Änderung in der Ablösestruktur ein großskalig inhomgenes Verhalten um 70 bis 75 Grad auf. Während bei $R e_{D}=1,5^{\times} 10^{5}$ (Bild 3.6 links) und $R e_{D}=1,8^{\times} 10^{5}$ (Bild 3.6 Mitte) sie eher spannweitig periodisch erscheinen, sind sie bei $\operatorname{Re}_{D}=2,1^{\times} 10^{5}$ (Bild 3.6 rechts) vergleichsweise nahezu spannweitig konstant. Damit kann geschlussfolgert werden, dass mit der topologischen Änderung der Ablösestruktur am Ende des unterkritischen Reynoldszahlbereiches eine spannweitig periodische Modulation der azimutalen Druckverteilung im Minimum, also bei etwa $75 \mathrm{Grad}$, verknüpft ist. Weiterhin scheint die spannweitige Länge dieser periodischen Struktur unabhängig von dem Streckungsverhältnis des Zylinders zu sein. Sowohl bei Higuchi et al. (siehe oben) und hier beträgt diese $y_{L}=2 D$. D.h., mit der Änderung der topologischen Ablösestruktur ist die Ausbildung einer spannweitig periodischen Struktur in der laminar ablösenden Grenzschicht verbunden. Hat sich die neue Ablösestruktur mit doppelter 
Ablöseblase vollständig ausgebildet, verliert sich auch die spannweitig periodische Variation im (azimutalen) Druckminimum.

Im folgenden bleibt zu klären, wie die Strukturänderung der ablösenden Grenzschichten mit der Transition in den freien abgelösten Scherschichten zusammenhängt. Die spannweitige Länge von $y_{L}=2 D$ deutet auf die Ausbildung großskaliger Wirbelstrukturen bzw. eines instabilen Verhaltens gegenüber spannweitig periodischen Störungen.

\subsection{Kritischer Übergangsbereich}

Im kritischen Reynoldszahlbereich vollziehen sich in kleinen Reynoldszahlausschnitten drastische Änderungen im zeitlich gemittelten wie im instationären Verhalten. Damit verknüpft sind Änderungen in der Struktur der abgelösten Zylinderströmung. Welche Instabilitäten diese räumlichen und zeitlichen Änderungen bewirken, wird mit Kraft- und Druckmessungen sowie durch Anstrichbilder diskutiert. Weiterhin weisen die abgelösten Zustände ein Hystereseverhalten auf. Schewe (1983) [75] erklärte dieses Verhalten durch eine subkritische Verzweigung. Da-

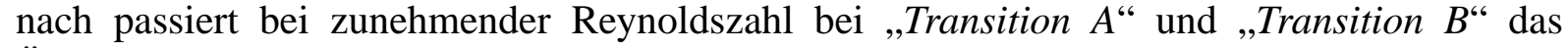
Übergangs-Szenario: stabil - instabil mit Auftreten niederfrequenter Fluktuationen - neuer stabiler Zustand; während dieses Szenario mit abnehmender Reynoldszahl bei „Transition $A^{* “ *}$ und „Transition $B{ }^{* “}$ ohne niederfrequente Fluktuationen begleitet wird.

\section{Zeitlich gemittelte Kraftmessungen}

Das zeitlich gemittelte Verhalten der abgelösten Strömung kann am Widerstands- und Auftriebsbeiwert abgelesen werden (Bild 3.7). Der asymmetrische Zustand mit einer transitionellen Ablöseblase auf der Oberseite des Zylinders wird bei zunehmender Reynoldszahl nur in einem kleinen Reynoldszahlausschnitt von $R e_{D}=3,2^{\times} 10^{5}$ bis $R_{D}=3,5^{\times} 10^{5}$ eingenommen. Trotzdem fällt der Auftriebsbeiwert von anfänglich $C_{A}=1,3$ auf $C_{A}=0,9 \mathrm{ab}$. Da umgekehrt mit der ,Transition $B *$ *“, mit abnehmender Reynoldszahl kein Anwachsen des Auftriebsbeiwertes zu beobachten ist, kann der Abfall auf die Wirkung einer Instabilität zurückgeführt werden. Eine Erklärung wäre, dass mit abnehmenden Auftriebsbeiwert sich auf der Unterseite des Zylinders ebenfalls eine transitionelle Ablöseblase ausbildet. Sie würde einen Auftrieb erzeugen, der dem ursprünglichen entgegenwirkt und somit den Gesamtauftrieb verringern. Beides würde eine Verkleinerung des Rezirkulationsgebietes und somit des Widerstandes bewirken. Eine signifikante Abnahme des Widerstandes innerhalb des asymmetrischen Reynoldszahlausschnittes tritt aber nicht auf (Bild 3.7 oben). Wahrscheinlicher ist, dass nach der „Transition A “ die turbulente Ablösung der Grenzschicht zunächst noch nahezu spannweitig homogen erfolgt, aber mit zunehmender Reynoldszahl eine spannweitig periodische Strukturierung einsetzt. Dies könnte durch das Instabilwerden der Ablöseblase auf spannweitig periodische harmonische Störungen verstanden werden. Theofilis et al. (2000) [90] und Hein et. al. (2000) [33] wiesen ein derartiges Verhalten für kleine Reynoldszahlen für die laminare Ablöseblase nach. Mit einem harmonischen Störansatz ergeben sich Instabilitätsmoden mit bestimmten spannweitigen Wellenlängen, die eine spannweitig periodische Ablösung erklären könnten. Mit der Ausbildung einer solchen Ablösestruktur wird die auftriebserzeugende Fläche verringert. Abschätzungen ergeben, dass durch diesen Effekt der Auftriebsbeiwert durchaus auf etwa zwei Drittel abfallen kann, ohne dass sich das Rezirkulationsgebiet in seiner Ausdehnung wesentlich ändert. Das räumliche Ablöseverhalten ist weiter mit Anstrichbildern und spannweitigen Druckverteilungen zu diskutie- 
ren. Darüberhinaus wird die asymmetrische Zylinderströmung global instabil gegenüber der Ausbildung eines neuen symmetrischen Zustandes. Mit der „Transition B“ wird dieser Zustand mit einer Ablöseblase auf jeder Seite des Zylinders eingenommen. Die Ablösung erfolgt nun wieder spannweitig homogen.

Das globale Verhalten der Ablösung äußert sich ebenso im instationären Verhalten. So fand Schewe (1983) [75] für die „Transition A“, dass zwei konkurrierende Zustände der Ablösung kurz vor dem Übergang existieren. Zum einen die ehemals dominante symmetrische Ablösung im unterkritischen Bereich mit $S r=0,16$ und zum anderen der neue, aber ebenfalls symmetrisch abgelöste Zustand des überkritischen Bereiches mit $S r=0,44$. Gleichzeitig treten niederfequente Fluktuationen auf, die als eine Eigenschaft eines instabilen Zustandes interpretiert werden (s.a. Haken 1992 [32]).
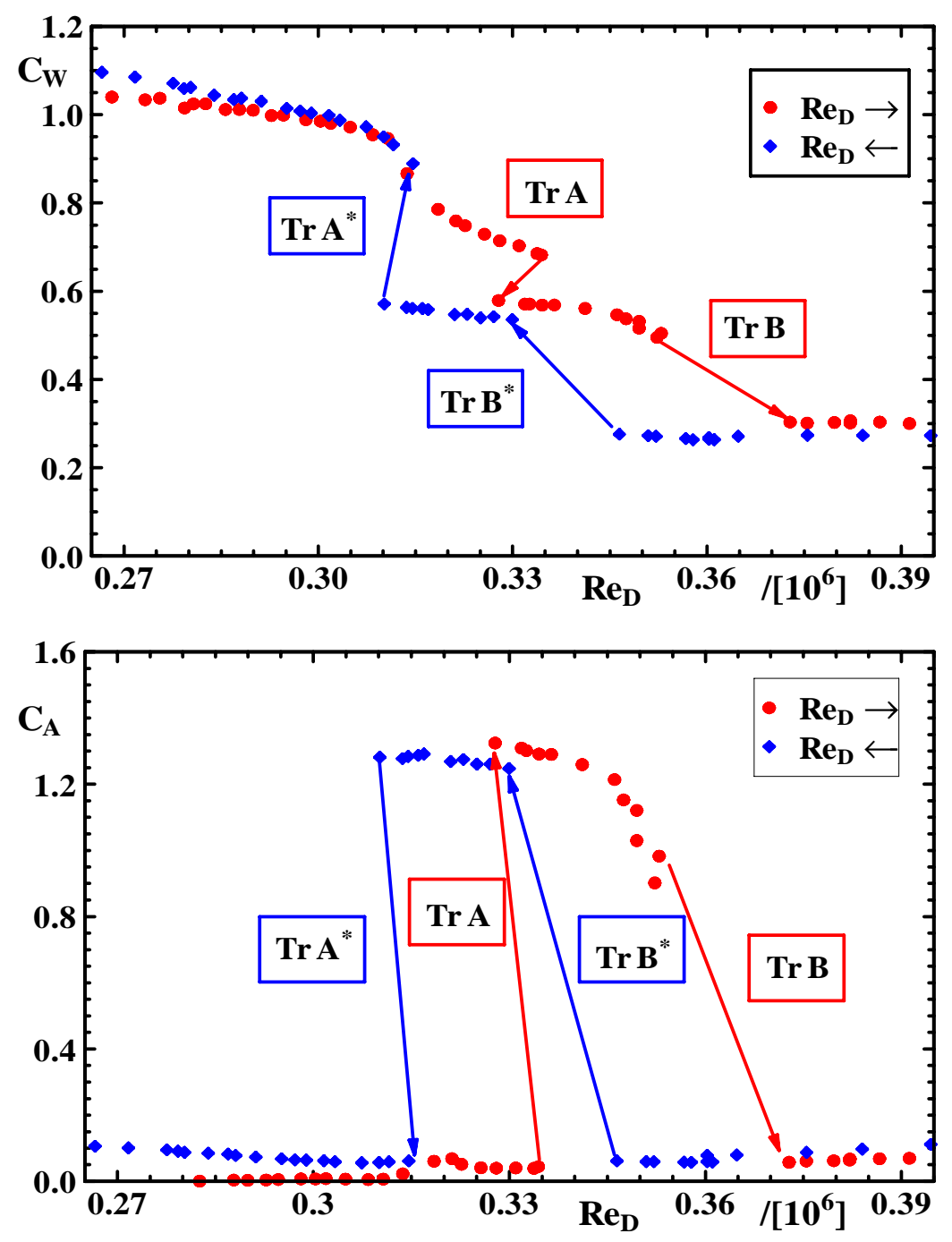

Bild 3.7 Verhalten von Widerstandsbeiwert (oben) und Auftriebsbeiwert (unten) im kritischen Übergangsbereich bei zunehmender und abnehmender Reynoldszahl in Abhängigkeit von der Reynoldszahl. Bei zunehmender Reynoldszahl treten vor der „Transition A“ und der „Transition B“ konkurrierende Zustände auf, die die jeweiligen Übergänge einleiten. Bei abnehmender Reynoldszahl treten diese konkurrierenden Zustände bei der „Transition $A^{* “}$ und „Transition $B^{* “}$ nicht auf. 


\section{Instationäre Kraftmessungen}

Mit der beidseitigen Ausbildung der doppelten Ablöseblase ab etwa $R e_{D}=2,0^{\times} 10^{5}$ nimmt der Widerstandsbeiwert kontinuierlich ab (Bild 3.7 oben). Dies ist verbunden mit einem Abfall der Schwankungen des Auftriebsbeiwertes bis zur „Transition A“. Wird der abgelöste Zustand bei etwas kleineren Reynoldszahlen als $R e_{k r i t, A}$ instabil, treten niederfrequente Fluktuationen auf, die Auftriebsschwankungen wachsen damit an und die Strouhalzahl wird kleiner. Dieses instationäre Bild ergibt sich ganz analog auch für die „Transition $B^{“(}$ (Bild 3.8). Bei abnehmender Reynoldszahl bei den Übergängen „Transition $A^{* “}$ und „Transition $B^{* “}$ werden diese Phänomene nicht beobachtet.
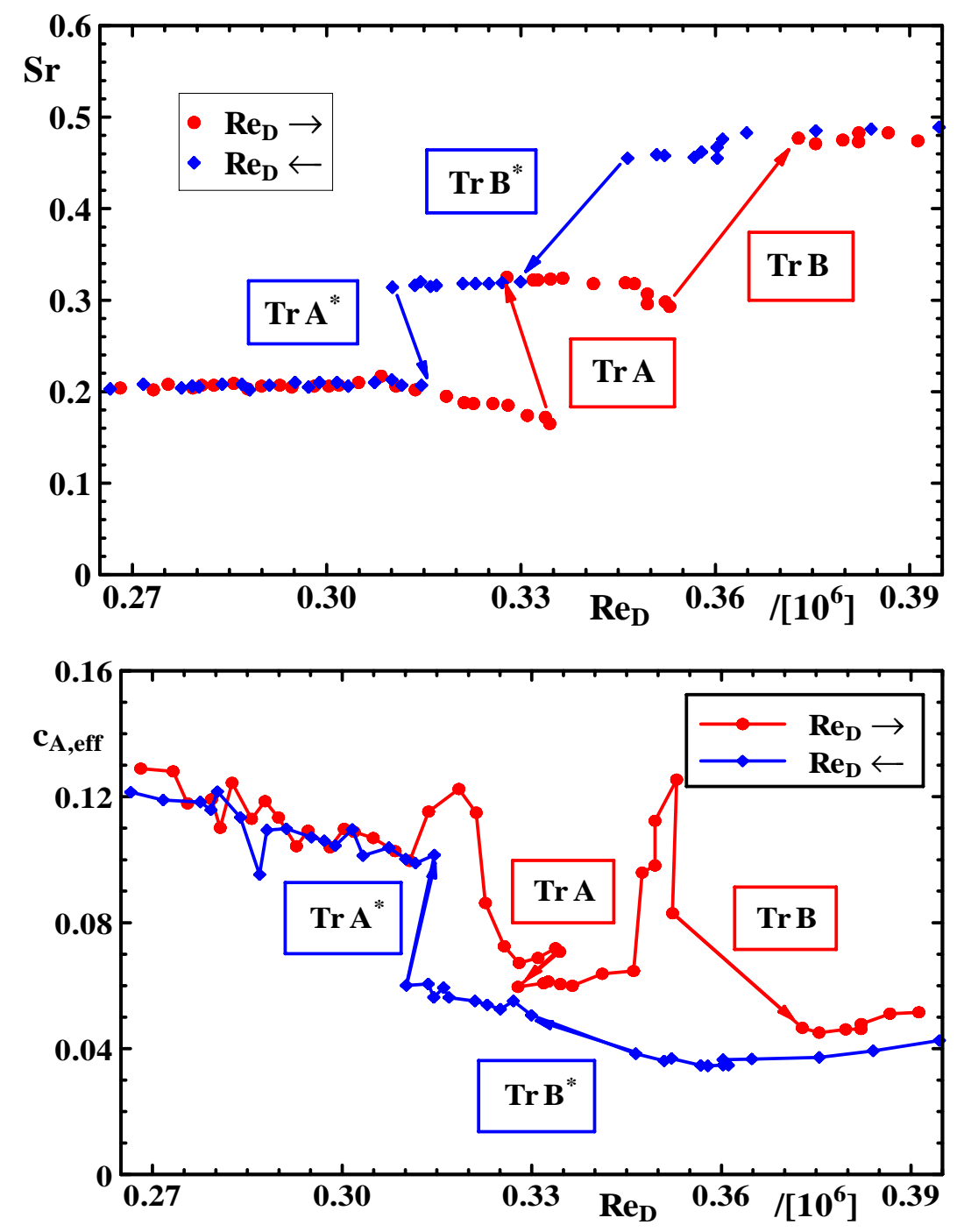

Bild 3.8 Verhalten der Strouhalzahl (oben) und des Effektivwertes der Schwankungen des Auftriebsbeiwertes (unten) im kritischen Übergangsbereich bei zunehmender und abnehmender Reynoldszahl. Vor den Übergängen „Transition $A$ “ und „Transition $B$ “ treten erhöhte Schwankungen im Auftriebsbeiwert auf, die mit einer geringen Abnahme der dominanten Strouhalzahl einhergehen. Umgekehrt bei abnehmender Reynoldszahl wird das entsprechende Verhalten nicht beobachtet. 
Die Entwicklung der Schwankungsamplituden bei den jeweiligen Strouhalzahlen (Bild 3.9) zeigen, dass die Amplitude bei ,Sr $=0,2$ “ sehr schnell kleiner wird und niederfrequente Schwankungen anwachsen. Weiterhin nimmt die Amplitude bei , $S r=0,5^{\text {“ }} \mathrm{zu}$ und besitzt während des konkurrierenden Zustandes die gleiche Amplitude wie bei „Sr $=0,2 “$. Ein analoges Verhalten der Wirbelablösung ist auch kurz vor der „Transition $B^{\prime \prime} \mathrm{zu}$ beobachten. Der asymmetrisch ab-

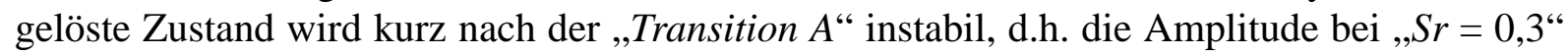
fällt ab, während gleichzeitig niederfrequente Fluktuationen auftreten und die Amplitude bei ,Sr $=0,5^{\prime \prime}$ anwächst. Auch hier erreichen die beiden konkurrierenden periodischen Ablösungen die gleiche Amplitude bevor die „Transition B“ erfolgt. Dieses Amplitudenkritirium für das Einsetzen des Überganges wird aber nur bei zunehmender Reynoldszahl festgestellt. Umgekehrt, bei abnehmender Reynoldszahl werden kurz vor dem Übergang keine niederfrequenten Fluktuationen und auch keine konkurrierenden Zustände gefunden.
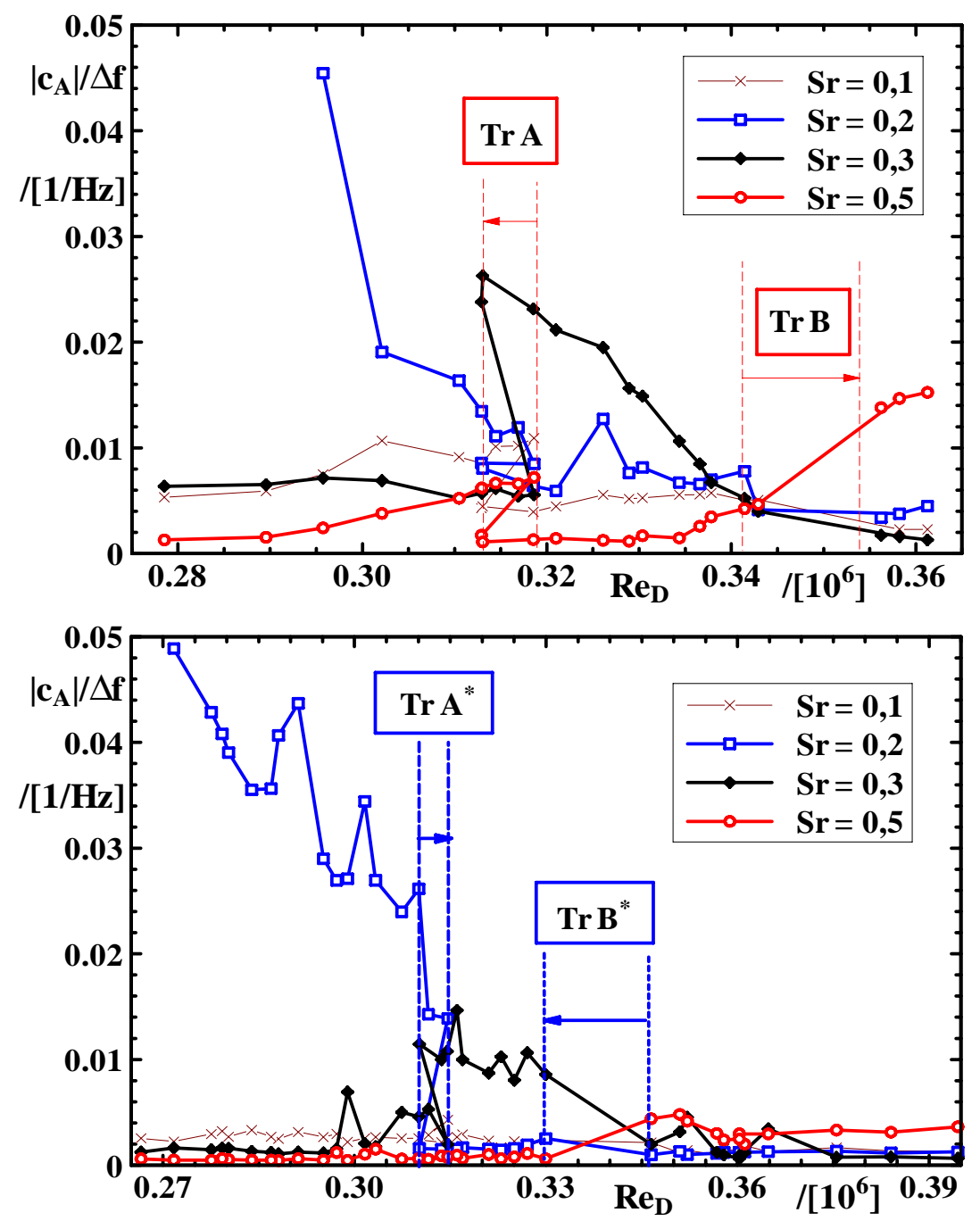

Bild 3.9 Verlauf der Amplituden der Auftriebsschwankungen für bestimmte Frequenzbereiche im kritischen Übergangsbereich bei zunehmender (oben) und abnehmender Reynoldszahl (unten). Bei zunehmender Reynoldszahl sind vor den jeweiligen Übergängen „Transition A“ und „Transition B“ stets zwei „,konkurrierende“ perodische Ablösungen zu beobachten. Bei abnehmender Reynoldszahl wird dieses Phänomen vor den Übergängen „Transition $A^{* “}$ und „Transition $B$ *“ nicht beobachtet. 

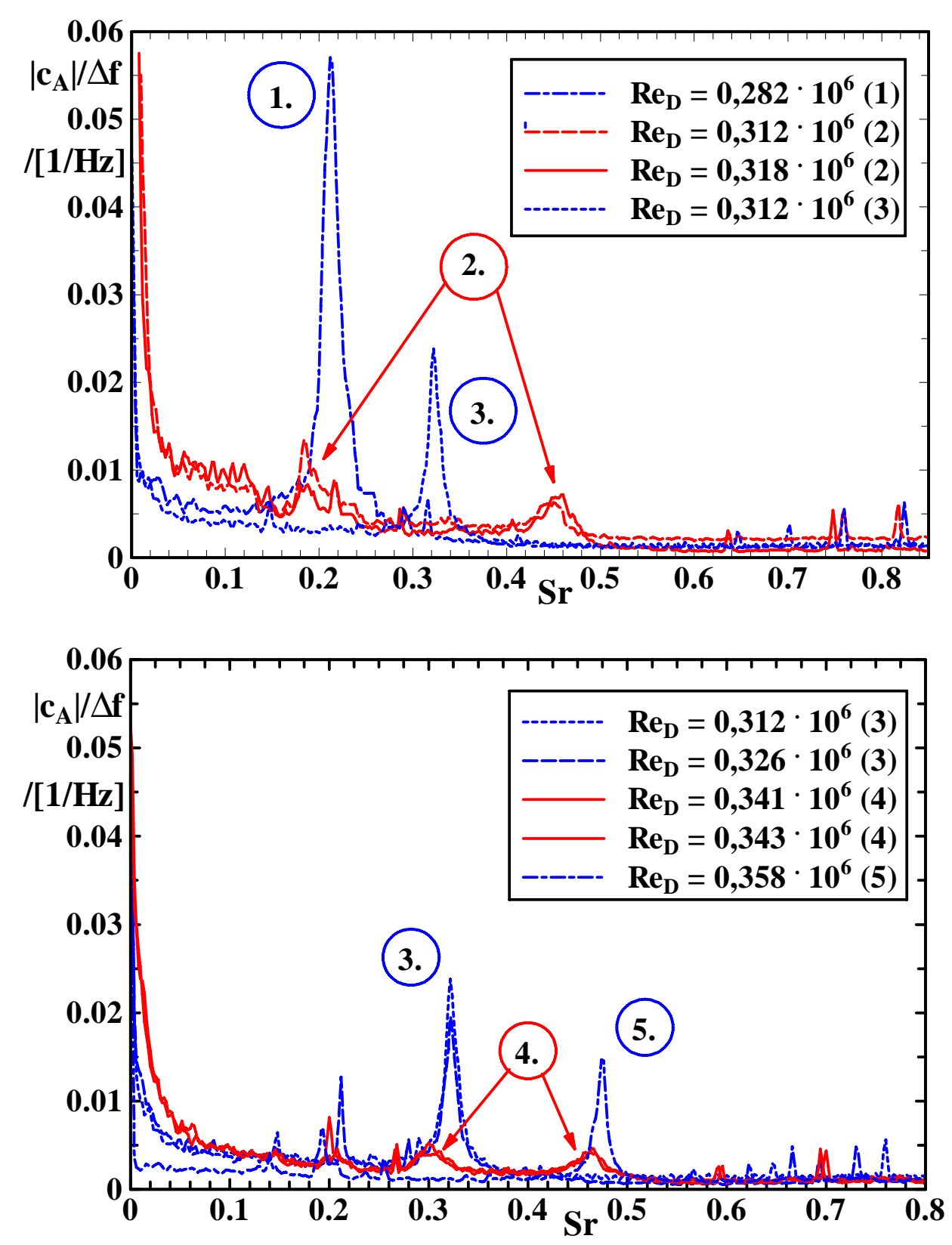

Bild 3.10 Charakteristische Amplitudenspektren des Auftriebsbeiwertes für verschiedene Reynoldszahlen vor und nach der „Transition A“ (oben) und vor und nach der „Transition $B^{\prime \prime}$ (unten) im kritischen Übergangsbereich bei zunehmender Reynoldszahl. (1) Dominante symmetrische Querwirbelablösung; (2) konkurrierender Zustand zwischen symmetrischer subkritischer und symmetrischer überkritischer Ablösung; (3) dominante asymmetrische Querwirbelablösung; (4) Konkurrierender Zustand zwischen asymmetrischer kritischer und symmetrischer überkritischer Ablösung; (5) dominante symmetrische Querwirbelablösung im superkritischen Bereich. 
Bei den Spektren in Bild 3.10 ist zu erkennen, dass die Amplitude der periodischen Ablösung kurz vor der „Transition A“ und „Transition B“ im Vergleich zu der im unterkritischen Bereich nahezu um eine Größenordnung gesunken ist (vgl. auch Schewe 1983 [75]). Beträgt der Effektivwert der Auftriebsschwankungen am Ende des unterkritischen Bereiches etwa $c_{A, \text { eff }} \approx 0,15$, hat sie in den konkurrierenden Zuständen nur einen Wert von etwa $c_{A, \text { eff }} \approx 0,015$. Die periodische Ablösung von Querwirbeln kann in diesen konkurrierenden Zuständen das Ablöseverhalten also nicht dominieren. Vielmehr muss eine andere Ablösestruktur mit den Übergängen verknüpft sein. Der symmetrisch abgelöste Zustand ist instabil und die Grenzschicht droht auf beiden Seiten des Zylinder turbulent abzulösen. Es sind die beiden symmetrischen Ablösezustände die vor der „Transition A “ miteinander konkurrieren. Andererseits können sich auf beiden Seiten nicht gleichzeitig die transitionelle Ablöseblase ausbilden. Vielmehr erfolgt zunächst die Ausbildung der Ablöseblase auf der einen Seite und stabilisiert damit sofort die laminare Ablösung auf der anderen Seite des Zylinders (Schewe 1983 [75]). Das Resultat ist eine azimutal asymmetrisch abgelöster Zustand, die eine periodische Ablösung mit der Strouhalzahl von etwa $S r=0,3$ hervorbringt. Erst mit weiterer Erhöhung des Ordnungsparameters, der Reynoldszahl, wird dieser asymmetrische gegenüber dem symmetrisch abgelösten Zustand mit beidseitig ausgebildeter Ablöseblase global instabil. Mit Hilfe von Anstrichbildern gilt es nachzuweisen, dass andere Ablösestrukturen als Querwirbel bei den kritischen Übergängen auftreten.

\section{Anstrichbilder}

Aus dem Verlauf des Auftriebsbeiwertes konnte mit der Ausbildung des asymmetrisch abgelösten Strömungszustandes ein Aball von $\left|C_{A}\right|=1,3$ auf $\left|C_{A}\right|=0,9$ festgestellt werden. Um zu zeigen, dass die zunächst spannweitig homogene Ablöseblase instabil gegenüber spannweitig periodischen Wirbelstrukturen wird, wurden kurz vor und nach der „Transition A“ Anstrichbilder angefertigt. Zu Beginn des kritischen Übergangsbereiches vor der „Transition A“ besteht noch sowohl auf der Ober- und auf der Unterseite des Zylinders die Ablösestruktur mit der doppelten Ablöseblase. Lediglich die azimutale Position der Ablöselinien befindet sich nun weiter stromab bei $82 \mathrm{Grad}$ und $98 \mathrm{Grad}$, wie es aus dem Anstrichbild bei $R e_{D}=3,2^{\times} 10^{5}$ ersichtlich ist (Bild 3.11). Die Ablöse-,,Linie“ ist zu den seitlichen Begrenzungen zu etwas größeren Azimutwinkeln gekrümmt, wo Randstörungen durch Hufeisenwirbel sich bemerkbar machen. Obwohl der gemessene mittlere Auftriebsbeiwert etwa Null ist, sind auf der Unterseite des Zylinders spannweitig lokal Farbvorträge bis zu einem Azimutwinkel von $135 \mathrm{Grad}$ zu beobachten. Die Ursache hierfür ist, dass mit Ende der Anfertigung des Anstrichbildes ein nichtverschwindender negativer Auftriebsbeiwert zu beobachten war. Der Widerstandbseiwert von $C_{W}=0,7$ weist dar-

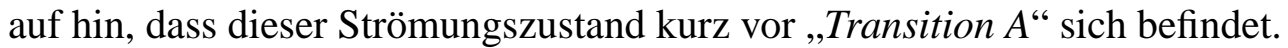

Auffällig bei diesem Anstrichbild ist die Form der ersten Ablöse-"Linie“. Spannweitig periodische Einschnürungen im Abstand von $\Delta y_{D}=0,14 D$ sind erkennbar. Eine Erklärung könnte dafür sein, dass kleinskalige Längswirbelstrukturen spannweitig periodisch ablösen bzw. in der abgelösten Scherschicht sich ausgebildet haben. Die o.g. spannweitige Länge liegt Wei und Smith 1986 [94] in der Größenordnung der spannweitigen Wellenlänge der Längswirbelstrukturen der freien abgelösten Scherschicht. Das bei der etwas größeren Reynoldszahl bei $\operatorname{Re}_{D}=3,4 \times 10^{5}$ angefertigte Anstrichbild (Bild 3.12) zeigt den asymmetrischen Charakter der abgelösten Zylinderströmung. Der Widerstandsbeiwert beträgt dabei $C_{W}=0,52$ und der mittlere Auftriebsbeiwert besitzt einen Wert von $C_{A}=-1,1$. 

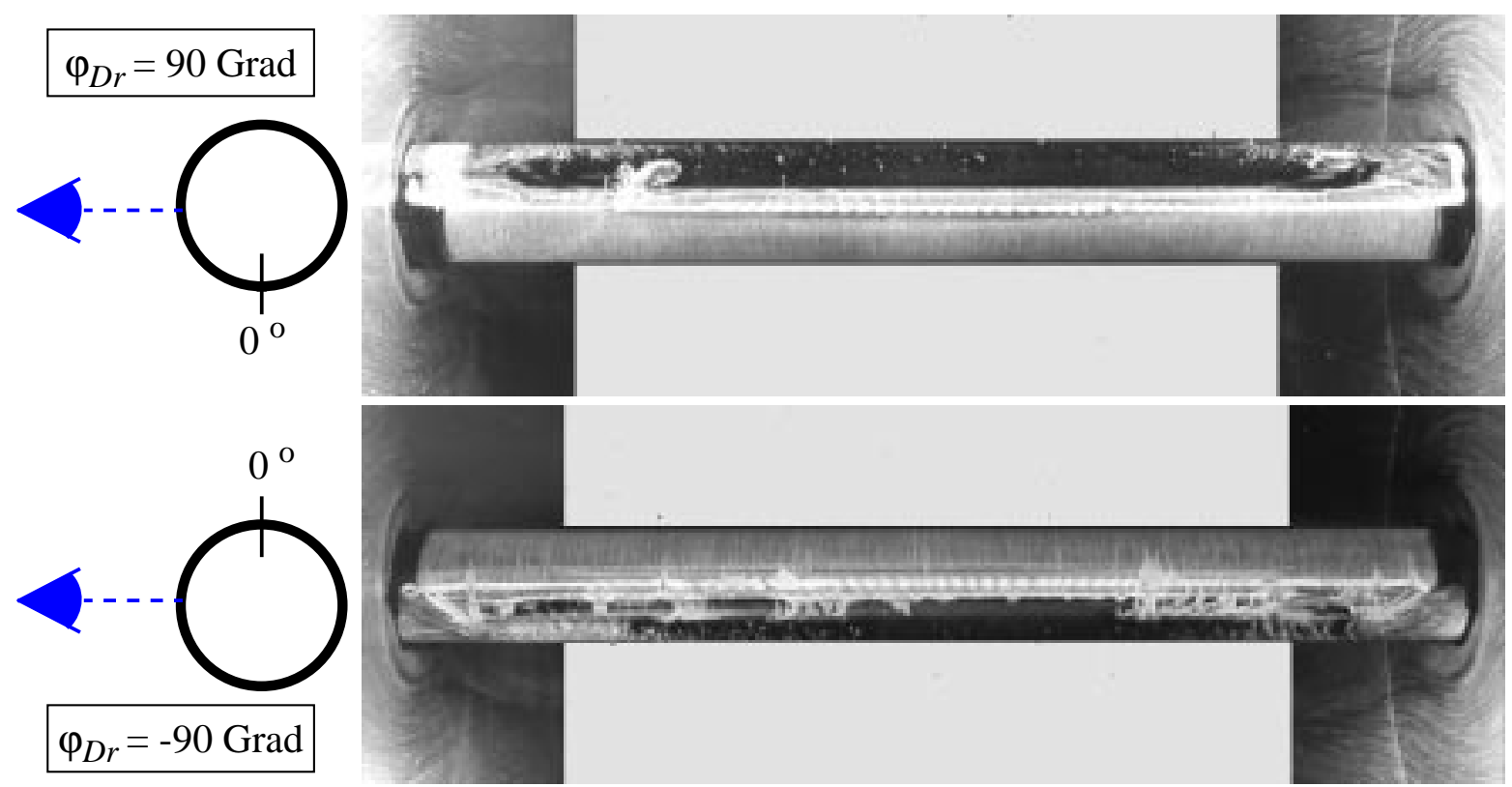

Bild 3.11 Anstrichbild bei $\operatorname{Re}_{\mathrm{D}}=3,2 \times 10^{5}$ mit einer Draufsicht unter einem Winkel von +90 Grad (oben) und -90 Grad (unten). Besonders im mittleren spannweitigen Bereich ist bei der ersten Ablöselinie auf Ober- und Unterseite eine spannweitig periodische Segmentierung von etwa $\Delta y_{D}=0,14 D$ zu erkennen (s.a. Bild A2.6).

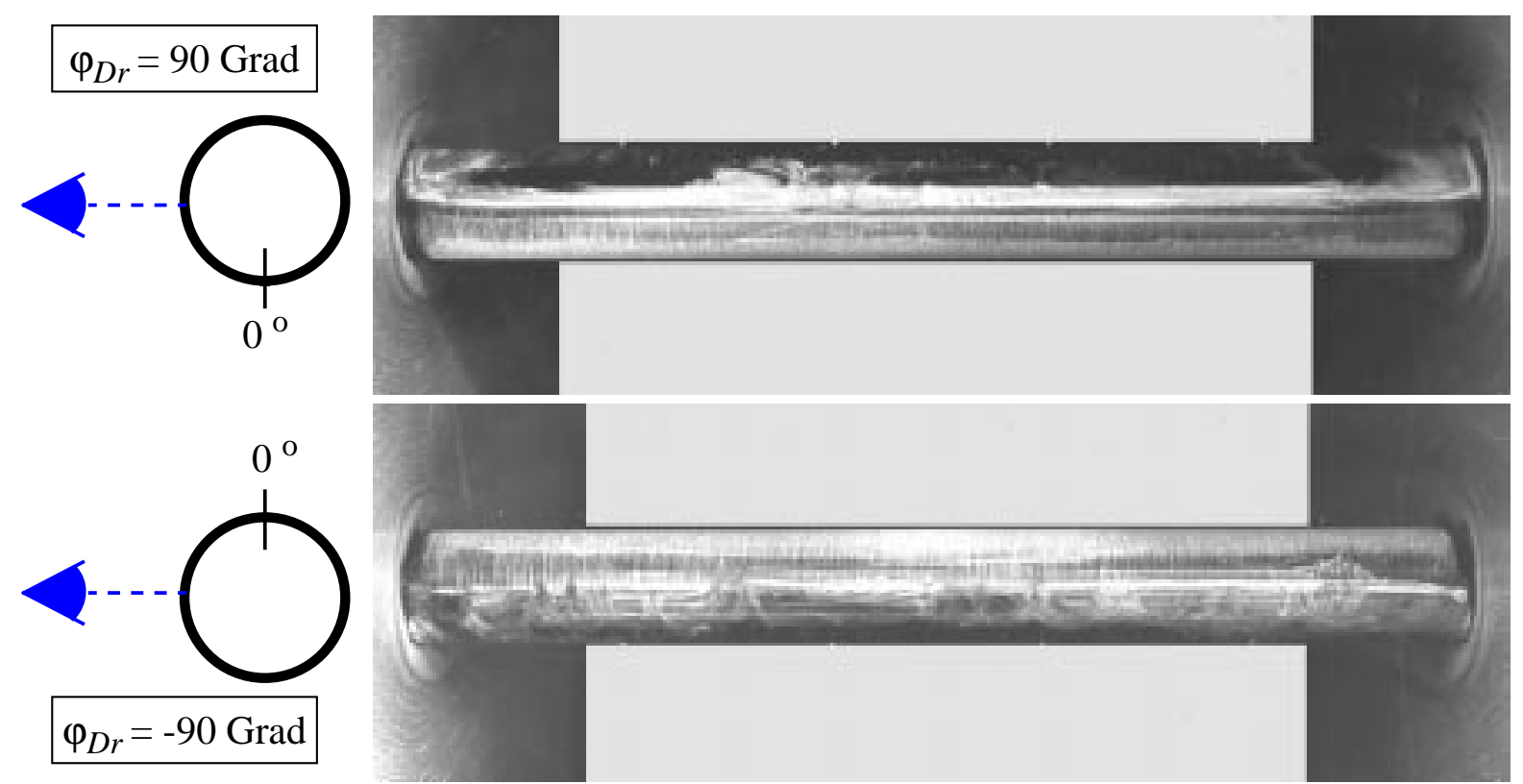

Bild 3.12 Anstrichbild bei $R e_{D}=3,4 \times 10^{5}$ mit einer Draufsicht unter einem Winkel von +90 Grad (oben) und -90 Grad (unten). Auf der Unterseite sind schwach drei Zellen einer spannweitig periodischen Struktur mit einer Länge von etwa $y_{L}=3 D$ zwischen 92 und 140 Grad festzustellen (s.a. Bild A2.7). 
Während auf der Oberseite des Zylinders also weiterhin die bekannte topologische Struktur der doppelten Ablöseblase zu beobachten ist, hat sich auf der Unterseite eine spannweitig inhomogene Ablösestruktur ausgebildet. Ein etwas verbreiteter Farbstreifen von etwa 85 Grad bis etwa 92 Grad markiert die spannweitige Ausbildung einer Ablöseblase. Weiter stromab sind etwas schwächer wieder Farbvorträge bis Azimutwinkeln von etwa 140 Grad festzustellen, die eine nahezu spannweitig periodische Struktur mit drei etwa gleich großen Zellen mit einer spannweitigen Länge von etwa $y_{L}=3 D$ erkennbar lassen. In diesem Abstand sind spannweitig lokale „Durchbrüche“ der Ablöseblase bei $y_{D}=1,3 ; 3 ; 6$ und 8,7 zu erkennen. An diesen Stellen bleibt die Strömung zunächst anliegend um erst weiter stromab mit der spannweitig benachbarten Strömung abzulösen. Aufgrund des damit sich ausbildenden spannweitigen Druckgradienten in der abgelösten Scherschicht kann es zur Ausbildung von Längswirbeln bzw. -paaren kommen (s.a. 3.4 Übergang vom überkritischen zum transkritischen Bereich).

Motiviert durch dieses Ergebnis wurde versucht, die spannweitig periodische Ablösestruktur auch in der Wanddruckverteilung wiederzufinden, so dass bei $\operatorname{Re}_{D}=3,3 \times 10^{5}$ die spannweitige Druckbeiwertverteilung in dem Azimutbereich von 60 Grad bis 125 Grad ermittelt wurde.

\section{Zeitlich gemittelte spannweitige Druckmessungen}

Die spannweitigen Druckbeiwertverteilungen für den Azimutwinkelbereich von 60 bis 125 Grad in Bild 3.13 wurden bei einer Reynoldszahl von $R e_{D}=3,3^{\times} 10^{5}$ gemessen, wobei diese durch Drehen des ,Aktiv“-Zylindermodells in 5 Grad Schritten erhalten wurden. Damit lässt sich das spannweitige Ablöseverhalten für diesen azimutal asymmetrischen Zustand weiter diskutieren und mit dem Anstrichbild bei $\operatorname{Re}_{D}=3,4 \times 10^{5}$ (Bild 3.12 ) auf dem ,Passiv“-Zylindermodell vergleichen.

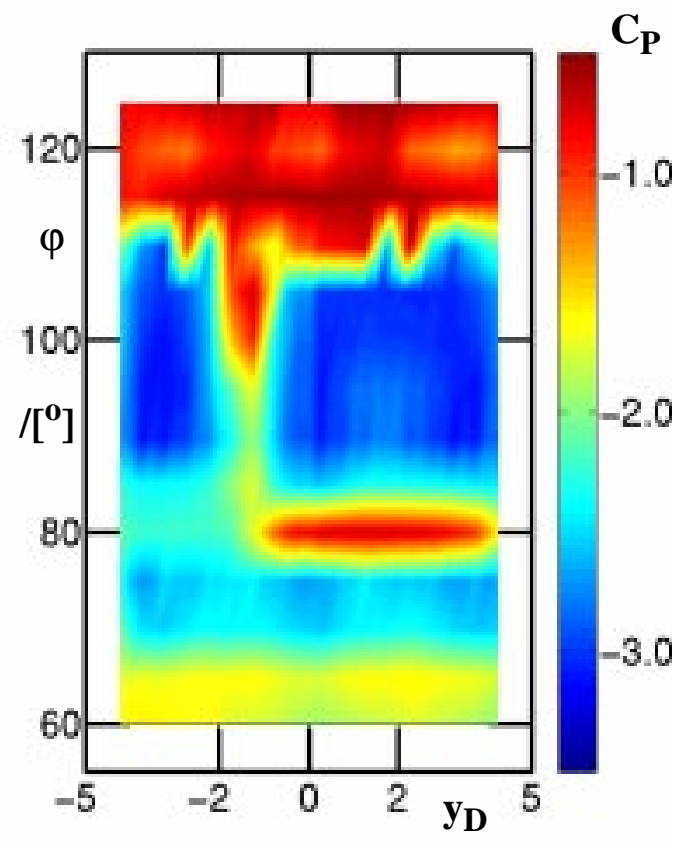

Bild 3.13 Spannweitige Druckverteilungen in dem azimutalen Bereich von 60 Grad bis 125 Grad bei $\operatorname{Re}_{D}=3,3 \times 10^{5}$ bei azimutal asymmetrischer Ablösung. Eine spannweitig periodische Variation des Druckbeiwertes tritt bei etwa 120 Grad auf. Der spannweitige Abstand der benachbarten Druckminima beträgt etwa $\Delta y_{D}=3$. Vergleiche dazu auch das Anstrichbild bei $\operatorname{Re}_{D}=3,3 \times 10^{5}$ in Bild 3.12. 
Mit dem azimutal asymmetrisch abgelösten Zustand ist bekanntermaßen die Ausbildung einer Ablöseblase auf einer Seite des Zylinders verbunden. Anhand der Druckverteilung für den gezeigten azimutalen Bereich muss sich diese zwischen $90 \mathrm{Grad}$ und $110 \mathrm{Grad}$ ausbilden, da hier azimutal das Druckminimum auftritt. Die Ablöseblase ist aber keinesfalls spannweitig homogen ausgebildet. Vielmehr wird diese bei einer Spannweite von etwa $y_{D}=-1$,,durchbrochen“, so dass zwei Blasen mit einer Länge von etwa $y_{L}=6 D$ und etwa $y_{L}=3 D$ existieren könnten. Andererseits könnte diese spannweitige Inhomogenität auch auf eine Störung in der Oberfläche zurückzuführen sein, die bereits bei $80 \mathrm{Grad}$ eine inhomogene spannweitige Druckverteilung verursacht. Bei diesem Azimutwinkel von 80 Grad ist die Grenzschicht am Zylinder laminar anliegend. Andererseits steht sie kurz vor der Ablösung, so dass die Wandschubspannung relativ klein ist und Störungen an der Wand die Grenzschicht turbulent werden lassen können. Vermutlich wird hier die Grenzschicht durch Druckbohrungen derart gestört, dass sie im spannweitigen Bereich von etwa $y_{D}=-1,0$ bis $y_{D}=+4,5$ turbulent ablöst. Dass die über der gesamten Spannweite gleichmäßig ausgeführten Druckbohrungen ein spannweitig unterschiedliches Ablöseverhalten bewirken, ist allerdings nicht verständlich. Es ist zwar auch für den anderen spannweitigen Bereich eine Verzögerung der Grenzschicht bei 80 Grad zu erkennen, aber sie bleibt laminar anliegend. Werden die Druckbohrungen zu größeren Azimutwinkeln gedreht, verursachen die Druckbohungen keine wesentlichen Störungen der ablösenden Grenzschicht.

Bei etwa 110 Grad bis 115 Grad stromab der Ablöseblase kommmt es zum Wiederanlegen der nunmehr turbulenten Grenzschicht und folgt zunächst der Körperkontur. Erst mit der weiteren Stromabentwicklung ist anhand der spannweitigen Druckverteilung zwischen 115 Grad und $125 \mathrm{Grad}$ deutlich eine spannweitig periodische Variation zu erkennen. Der spannweitige Abstand der lokalen Druckminima beträgt etwa $\Delta y_{D}=3$ und entspricht damit in guter Weise der im Anstrichbild (Bild 3.12) gefunden spannweitig periodischen Ablösestruktur.

Damit kann ausgesagt werden, dass im kritischen Bereich die ablösende Grenzschicht instabil gegenüber spannweitig periodischen Wirbelstrukturen ist. Dieses Verhalten könnte auf eine Instabilität der sich zunächst spannweitig homogen ausbildenden Ablöseblase zurückgeführt werden. So fand z.B. Owen et al. (2000) [58] an einem Zylindermodell eine spannweitige Wellenlänge von $y_{L}=5,6 D$. Auch numerische Untersuchungen von Darekar et al. (1999) [22] an einem gewellten Zylinder zeigen, dass spannweitig periodische Moden unterschiedlicher Wellenlänge unterschiedlich angefacht auftreten. Die am stärksten angefachte Mode besitzt ebenfalls eine spannweitige Wellenlänge von $y_{L}=5,6 \mathrm{D}$. Numerische Instabilitätsuntersuchungen an laminaren Ablöseblasen von Theofilis et al. (2000) [90] kommen zu einem ähnlichen Ergebnis. Durch Aufprägung einer spannweitig harmonischen Störung werden bestimmte spannweitige Moden aufgrund einer so genanten ,globalen Instabilität“" angefacht. Das Phänomen der Ausbildung spannweitig periodischer Wirbelstrukturen in Verbindung mit der Ausbildung von Ablöseblasen lässt vermuten, dass auch die von Humphreys (1960) [37] bei $R e_{D}=$ $3,4 \times 10^{5}$ und von Dallmann und Schewe (1987) [20] bei $R e_{D}=4,1^{\times} 10^{5}$ im überkritischen Reynoldszahlbereich gefundenen spannweitig periodischen Wirbelstrukturen mit dem Instabilitätsverhalten der Ablöseblasen verknüpft sind. Da zuvor im unterkritischen Bereich die Änderung der Ablösestruktur zur doppelten Ablöseblase ebenfalls mit der Ausbildung spannweitig periodischen Wirbelstrukturen in Verbindung gebracht wurde, scheint das lokale Instabilitätsverhalten der ablösenden Grenzschicht in Wechelwirkung mit den freien abgelösten Scherschichten das Ablöseverhalten der Zylinderströmung global zu beeinflussen. 


\section{4 Übergang vom überkritischen zum transkritischen Bereich}

Während im überkritischen Bereich der Widerstandsbeiwert auf einen minimalen Wert von etwa $C_{W}=0,2$ verharrt, ist der Beginn des oberen Übergangsbereiches durch das Anwachsen des Widerstandsbeiwertes bis auf einen höheren Wert im transkritischen Bereich auf $C_{W}=0,5$ gekennzeichnet. Verbunden damit sind auch Änderungen im räumlichen wie im instationären Ablöseverhalten.

Analog zum kritischen Übergangsbereich lassen sich auch im oberen Übergangsbereich Aussagen über das spannweitige Ablöseverhalten aus den zeitlich gemittelten Druckverteilungen und mit Hilfe von Anstrichbildern gewinnen. Für die Beschreibung der Änderungen des instationären Ablöseverhaltens werden wieder mit Hilfe von Kraftmessungen die Entwicklung der Betragsamplituden $\mathrm{zu}$ den Ablösefrequenzen in Abhängigkeit von der Reynoldszahl und zugehörige Spektren des Auftriebs herangezogen.

Es wird sich zeigen, dass spannweitig periodische Längswirbelstrukturen das räumlich-zeitliche Ablöseverhalten im oberen Übergangsbereich bestimmen. Sie sind vergleichbar mit denen, die Humphreys (1960) [37] mit Verlassen des kritischen Übergangsbereiches mit Hilfe von Seidenfäaden erstmals gefunden hatte. Darüberhinaus untermauern die topologischen Untersuchungen von Dallmann und Schewe (1987) [20] diese Beobachtungen und zeigen ein Anstrichbild, welches spannweitig periodisch abgelöste Strukturen am Kreiszylinder bei einer Reynoldszahl von $R e_{D}=4,1 \times 10^{5}$.

\section{Anstrichbilder}

Neben den Anstrichbildern bei den Reynoldszahlen von $R e_{D}=0,41 \times 10^{6}$ (Bild 3.15), $R e_{D}=$ $0,59 \times 10^{6}$ (Bild 3.16) und $R e_{D}=0,79 \times 10^{6}$ (Bild 3.17) wurden noch weitere Anstrichbilder bei

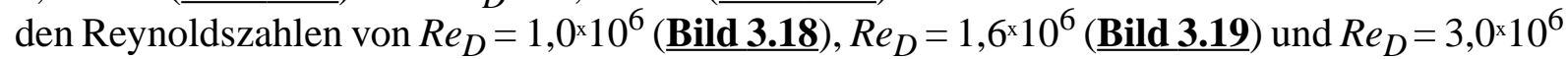
(Bild 3.20) angefertigt.

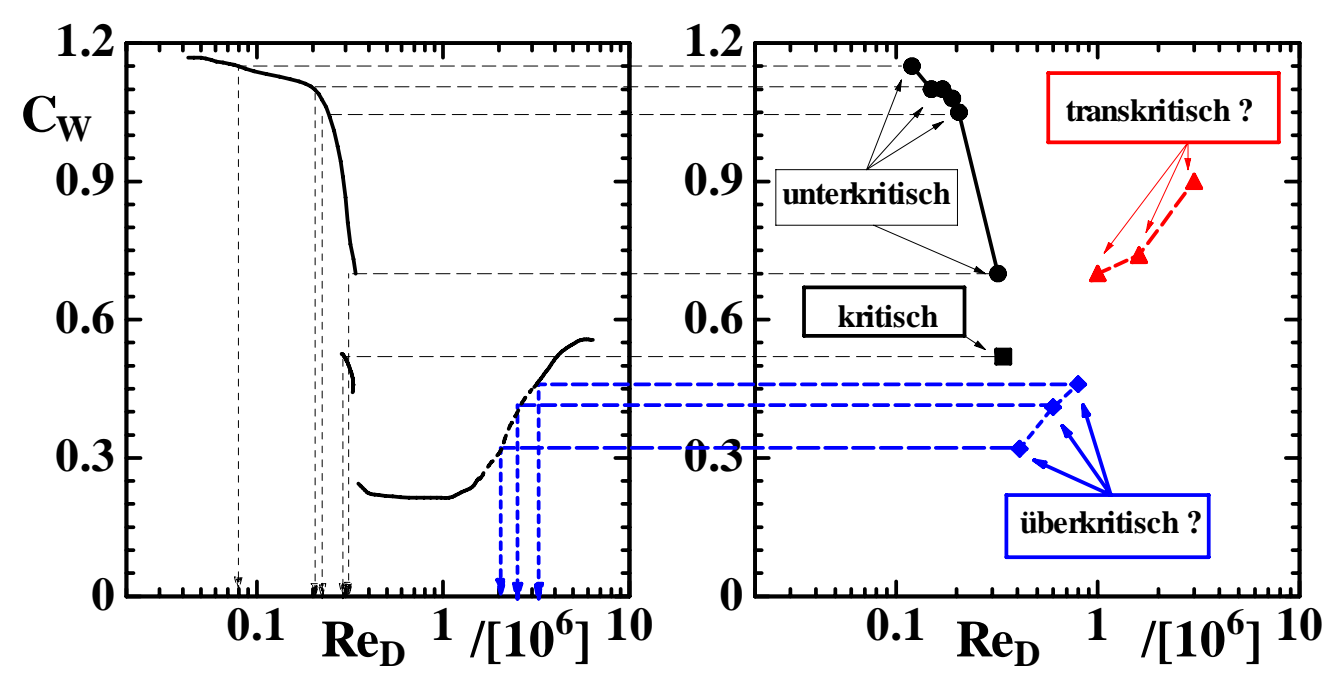

Bild 3.14 Zuordnung der Strömungszustände in den Anstrichbildern zu den Bereichen für eine Zylinderströmung mit glatter Oberfläche über den Widerstandsbeiwert. Der überkritische Bereich erweist sich dabei als instabil gegenüber Störungen auf der Oberfläche des Zylinders, die die Anstrichfarbe darstellt. Die Anstrichbilder bei den gemessenen Reynoldszahlen größer als ab $R e_{k r i t, B}$ sind somit Zuständen des oberen Übergangsbzw. transkritischen Bereiches zuzuordnen. 
Obwohl die Reynoldszahlen zu dem ersten Tripel der Anstrichbilder dem überkritischen Bereich und das zweite Tripel eher dem oberen Übergangsbereich angehören, können in diesen Anstrichbildern Strömungzustände des oberen Übergangsbereiches bis zum transkritischen Bereich identifiziert werden. D.h., dass eine Verschiebung der gemessenen Reynoldszahlen zu entsprechenden größeren Reynoldszahlen für den Vergleich mit einer Zylinderströmung mit glatter Oberfläche durchzuführen ist. Die Begründung dafür ist im instabilen Verhalten der Grenzschicht gegenüber Rauhigkeiten zu suchen. Die Bestimmung der zugehörigen Reynoldszahl ergibt sich über den Vergleich des bei dem Anstrichbild ermittelten Widerstandsbeiwertes mit dem Widerstandsbeiwert am glatten Zylinder. Damit können die Strömungszustände in den Anstrichbildern des ersten Tripels dem oberen Übergangsbereich, während die Anstrichbilder des zweiten Tripels eher dem transkritischen Bereich zuzuordnen sind (Bild 3.14). So sind tatsächlich bei einer gemessenen Reynoldszahl von $R e_{D}=0,41 \times 10^{6}$ im Anstrichbild (Bild 3.15) spannweitig periodische zellartige Ablösestrukturen zu beobachten. Längs der Spannweite sind vier vollständig ausgebildete zellartige Ablösestrukturen nahezu symmetrisch zur Symmetrieebene des Zylinders beidseitig in einem Azimutwinkelbereich von etwa 102 bis 137 Grad zu erkennen. Darüberhinaus ist noch jeweils eine halbe Zelle an den seitlichen Begrenzungen des Zylinders vorhanden. Durch die Wirkung des Rückstromgebietes zeichnen sich im Heckbereich des Zylinders die dazugehörigen spannweitig verteilten fünf Staudruck-Zellen ab. Die Länge einer Zelle beträgt dabei etwa $y_{L}=1,7 \mathrm{D}$. Der Widerstandsbeiwert beträgt hierbei $C_{W}=0,32$, während der Auftriebsbeiwert im Mittel verschwindet. Die zu diesem Widerstandsbeiwert korrespondierende Reynoldszahl für eine abgelöste Zylinderströmung mit glatter Oberfläche beträgt etwa $R e_{D}=1,1^{\times} 10^{6}$, womit ein Zustand des oberen Übergangsbereiches vorliegt. Eine dominante Ablösefrequenz konnte nicht gefunden werden, vielmehr dominieren Fluktuationen mit $S r<0,2$ das instationäre Ablöseverhalten.

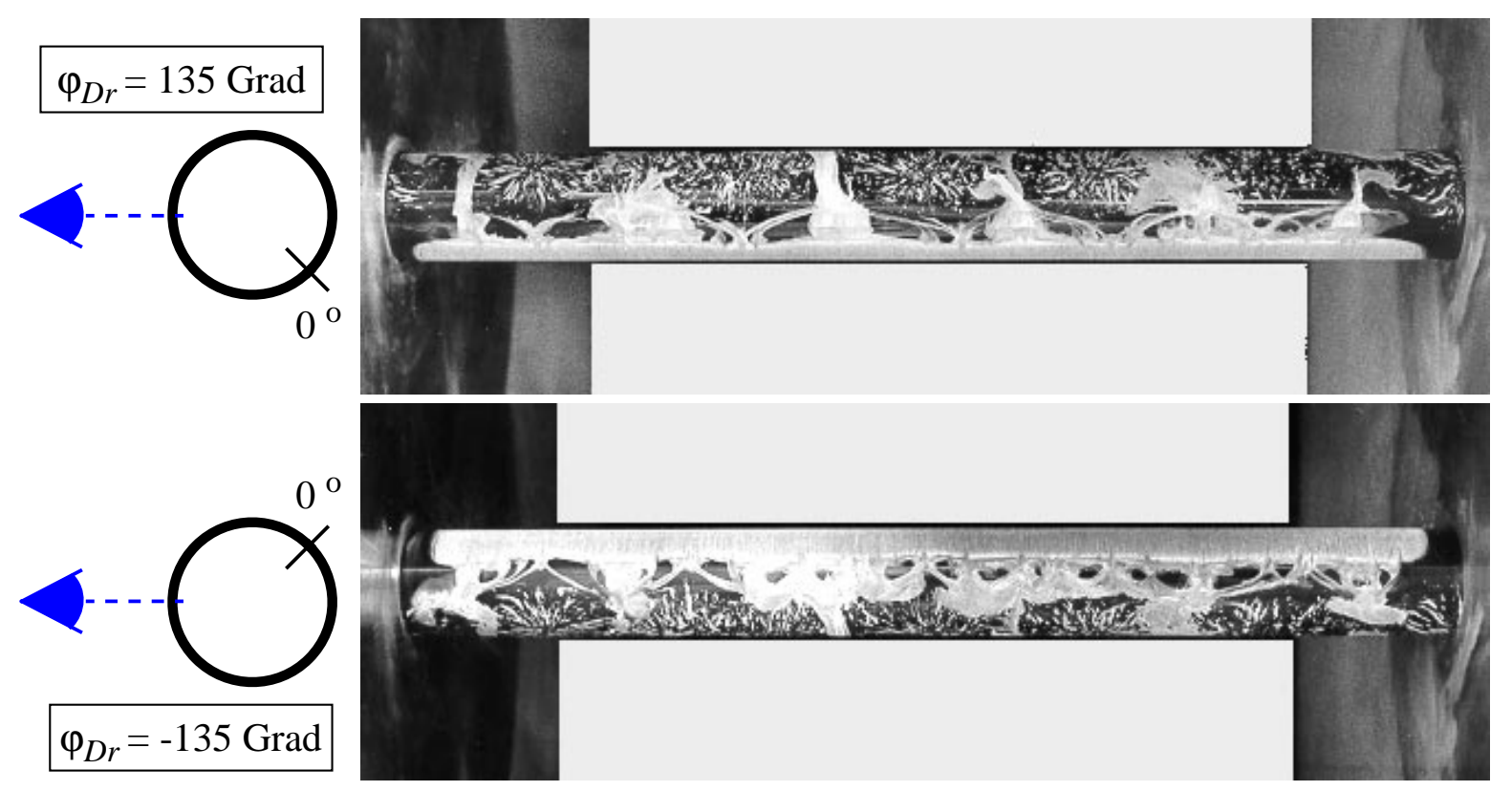

Bild 3.15 Anstrichbild bei $R e_{D}=4,1 \times 10^{5}$ mit einer Draufsicht unter einem Winkel von +135 Grad (oben) und -135 Grad (unten). Ausbildung von vier großen spannweitig periodischen zellartigen Ablösestrukturen auf jeweils jeder Seite des Zylinders (s.a. Bild A2.8). 


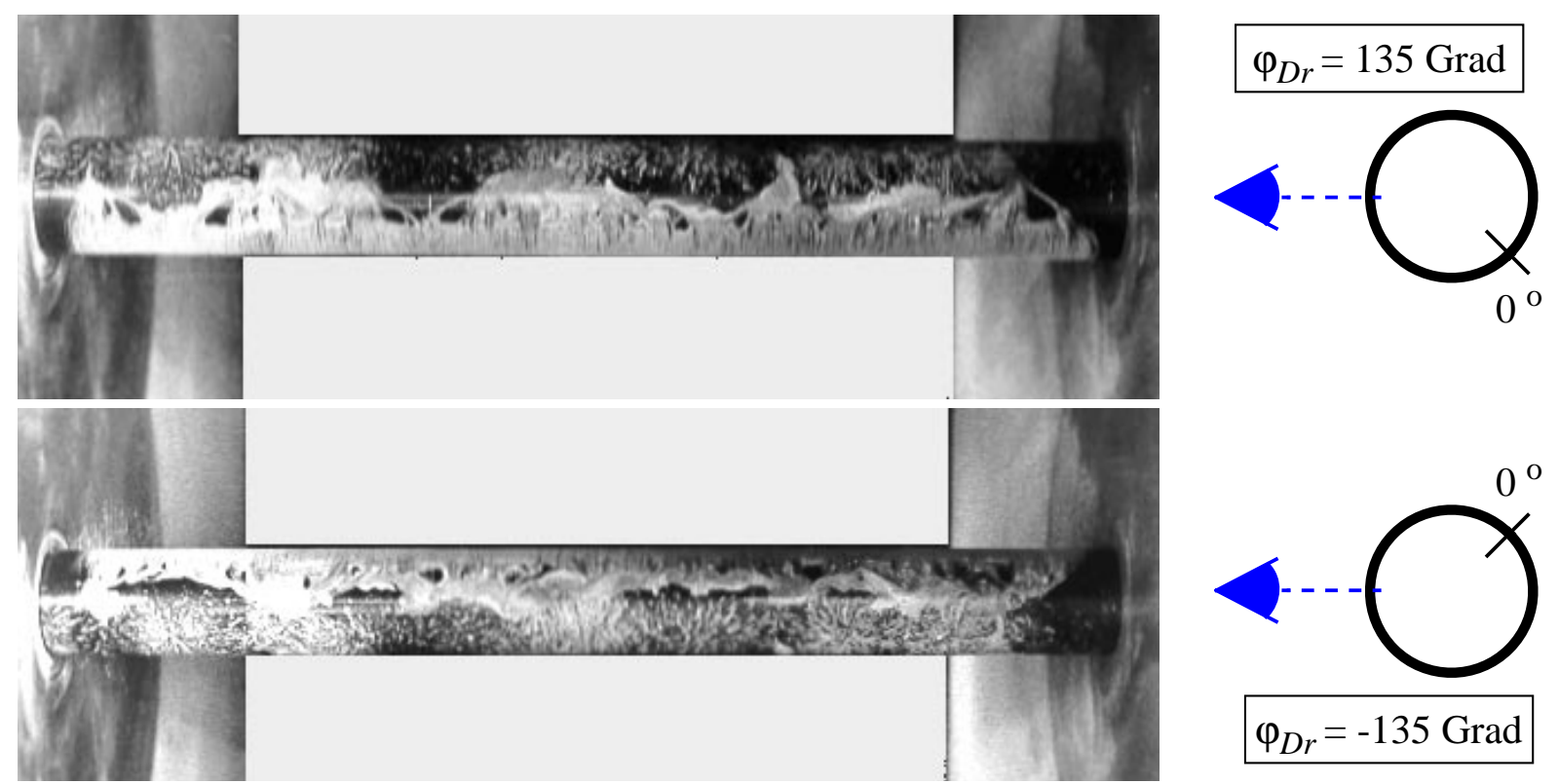

Bild 3.16 Anstrichbild bei $\operatorname{Re}_{\mathrm{D}}=0,59 \times 10^{6}$ mit einer Draufsicht unter einem Winkel von +135 Grad (oben) und -135 Grad (unten). Ausbildung von 3 zellulären Ablösestrukturen, wobei die auf der Innenseite des Windkanals (hier: rechts von der Zylindermitte) etwas schächer ausgeprägt ist (s.a. Bild A2.9).
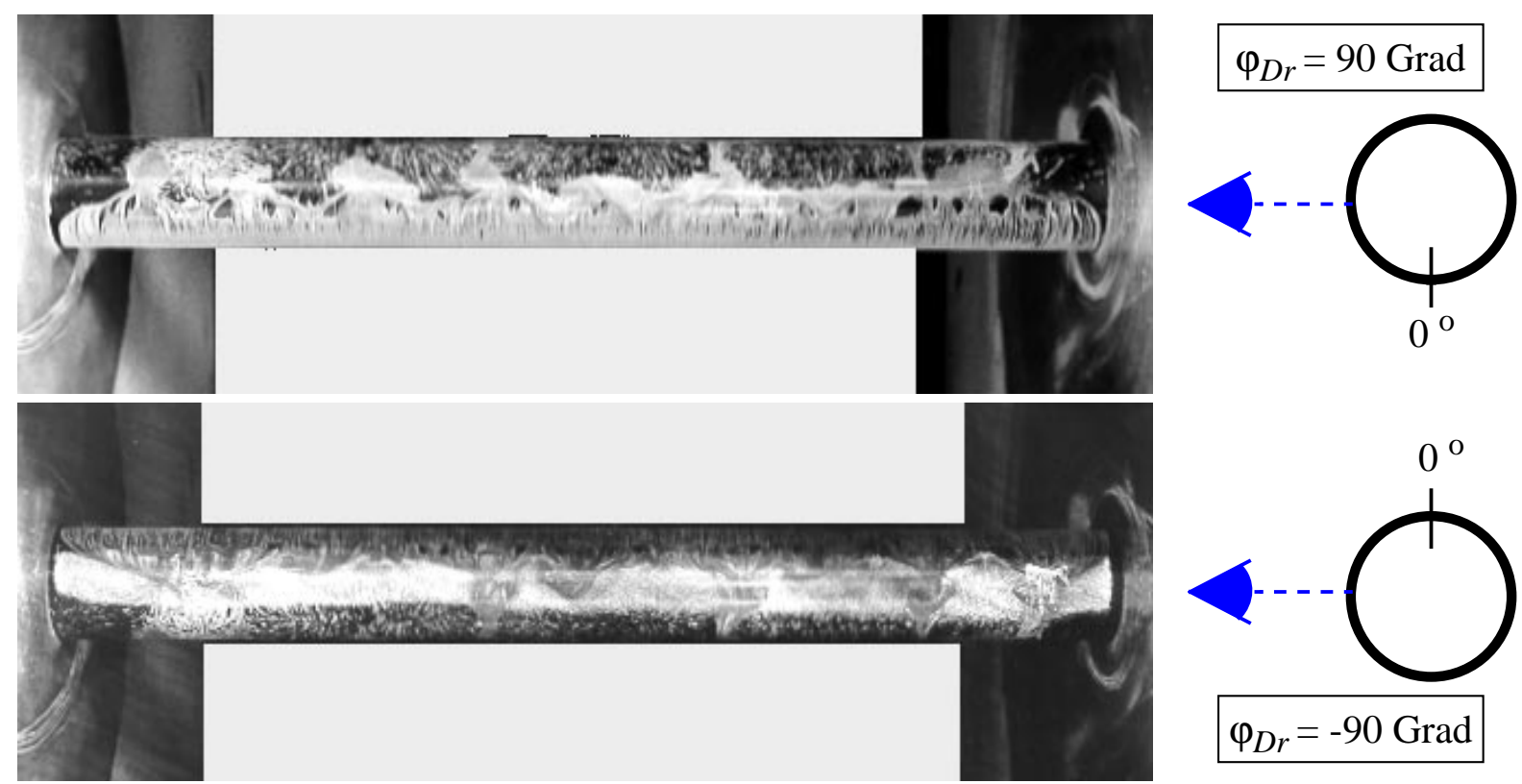

Bild 3.17 Anstrichbild bei $\operatorname{Re}_{D}=0,79 \times 10^{6}$ mit einer Draufsicht unter einem Winkel von +90 Grad (oben) und -90 Grad (unten). Ausbildung von 8 zellulären Ablösestrukturen jeweils auf jeder Seite des Zylinders (s.a. Bild A2.10).

Der Vergleich der Visualisierung der Ablösestruktur einer Zelle im Anstrichbild mit der von Humphreys (1960) [37] mittels Seidenfäden liefert eine geometrische Ähnlichkeit. Die Länge einer Zelle skaliert dabei mit dem Zylinderdurchmesser und beträgt zwischen $y_{L}=1,4 D$ bis 
$y_{L}=1,7 D$. Obwohl die gemessene Reynoldszahl von $R e_{D}=3,29 \times 10^{5}$ etwas kleiner ausfällt, muss mit dieser Reynoldszahl bereits der überkritische Zustand erreicht sein. Der mit Seidenfäden beobachtete Widerstandsbeiwert beträgt $C_{W}=0,37$. Da das zelluläre Muster in dem Anstrichbild bei $\operatorname{Re}_{D}=0,41 \times 10^{6}$ dem des durch die Seidenfäden gebildete sehr ähnlich ist und die Längen einer Zelle in beiden Visualisierungen in etwa übereinstimmen, kann geschlossen werden, dass in beiden Fällen ein gleicher Strömungszustand vorliegt. Das wiederum bedeutet, dass die spannweitig verteilte Rauhigkeit durch die Seidenfäden in dem azimutalen Ablösebereich von $100 \mathrm{Grad}$ bis $140 \mathrm{Grad}$ genau wie die mehr oder weniger stochastisch verteilte Rauhigkeit auf der Zylinderoberfläche durch die Anstrichfarbe eine identische Wirkung auf das spannweitige Ablöseverhalten hat. D.h., dass die Verteilung der oberflächlichen Störungen für die Ausbildung der Ablösestruktur bestimmter spannweitiger Länge keinen Einfluss zu haben scheint (s.a. 4.1 Spannweitig-periodische stationäre Störung mit Wirbelerzeugern).

Bei den anderen beiden Anstrichbildern (Bild 3.16 und Bild 3.17) ist nach wie vor ein mehr oder weniger spannweitig periodisches Ablöseverhalten zu erkennen, obwohl die Strukturen nicht mehr so deutlich ausgeprägt sind. So sind im Anstrichbild bei $\operatorname{Re}_{D}=0,59 \times 10^{6}(\underline{\text { Bild 3.16}})$ sieben Staudruck-Zellen im Heck des Zylinders abzählbar. Andererseits können auf der Oberseite des Zylinders nur drei zellartig verteilte Wirbelstrukturen bei etwa $y_{D}=-2,5$ und $y_{D}=0,0$ sowie schwächer ausgeprägt bei $y_{D}=+2,5$ identifiziert werden. Bei der noch größeren Reynoldszahl bei $R e_{D}=0,79 \times 10^{6}$ entsteht eine andere Ablösestruktur (Bild 3.17). Die mittlere Länge einer Zelle beträgt hier im Mittel $y_{L}=1,2 D$. D.h., acht Wirbelstrukturen sind zu erkennen, wobei die auf der Innenseite der Messstrecke etwas schwächer ausgeprägt sind.

Mit diesen drei Anstrichbildern aus dem oberen Übergangsbereich werden spannweitig periodische, abgelöste Wirbelstrukturen identifiziert. Die zunächst spannweitig homogen ausgebildete Ablöseblase auf jeder Seite des Zylinders wird mit zunehmender Reynoldszahl instabil gegenüber spannweitig periodischen Wirbelstrukturen. In Abhängigkeit von der Reynoldszahl bilden sich Strukturen mit bestimmten spannweitigen Wellenlängen aus. Dabei sei eine Wellenlänge die spannweitige Länge einer Zelle der Ablösestruktur. Zu Beginn des oberen Übergangsbereiches beträgt die Wellenlänge etwa $2 D$, mit weiter zunehmender Reynoldszahl etwa $3 D$ und zum Ende etwa 1,2D. Vermutlich besteht die Wirbelstruktur je Zelle aus paarweise ablösenden Längswirbeln. Mit weiterer Verringerung der spannweitigen Länge einer solchen Wirbelstruktur kommt es mit der Stromabentwicklung zu einer zunehmenden Verwirbelung benachbarter Längswirbel (Bushnell 1992 [18]). Mit Erreichen einer bestimmten oberen kritischen Reynoldszahl wird die spannweitig periodische Längswirbelstruktur gegenüber der Ausbildung von periodisch ablösenden Querwirbeln instabil. Dieser Übergang ist verbunden mit einem Ansteigen der Schwankungen des Auftriebsbeiwertes, wie es in Bild 3.1 bei $R e_{D}=2,1^{\times} 10^{6} \mathrm{zu}$ sehen ist. Bei noch größeren gemessenen Reynoldszahlen ab $R e_{D}=1,0^{\times} 10^{6}$ ist in den Anstrichbildern keine spannweitig periodisch Ablösestruktur zu erkennen. Die Ablösung erfolgt nun wieder nahezu spannweitig homogen, wobei darauf gleich noch genauer eingegangen wird.

Bei Betrachtung des Anstrichbildes bei der gemessenen Reynoldszahl von $R e_{D}=1,0^{\times} 10^{6}(\underline{\text { Bild }}$ 3.18) sind auf der Ober- und Unterseite des Zylinders zwei Ablöselinien festzustellen. Während die eine Ablöselinie sich über der gesamten Spannweite des Zylinders bei einem Azimutwinkel von etwa 89 Grad ausgebildet hat, ist die zweite, weiter stromab liegende Ablöse-"Linie" bei einem Azimutwinkel von etwa 110 nur über einen spannweitigen Bereich von $y_{D}=-3$ bis $y_{D}=$ +3 ausgeprägt. Mit weiter zunehmender Reynoldszahl (siehe Bild 3.19 und Bild 3.20) wandert die erste Ablöselinie etwas weiter stromauf und befindet sich bei einem Azimutwinkel von etwa 
92 Grad bei der gemessenen Reynoldszahl $R e_{D}=1,60 \times 10^{6}$ und bei etwa 88 Grad bei der gemessenen Reynoldszahl $R e_{D}=3,0^{\times} 10^{6}$, während die zweite Ablöselinie in den Anstrichbildern nicht mehr zu beobachten ist. Lediglich bei der gemessenen Reynoldszahl von $R e_{D}=1,6^{\times} 10^{6}$ ist stromab der ersten Ablöselinie ein Farbvortrag zu erkennen.

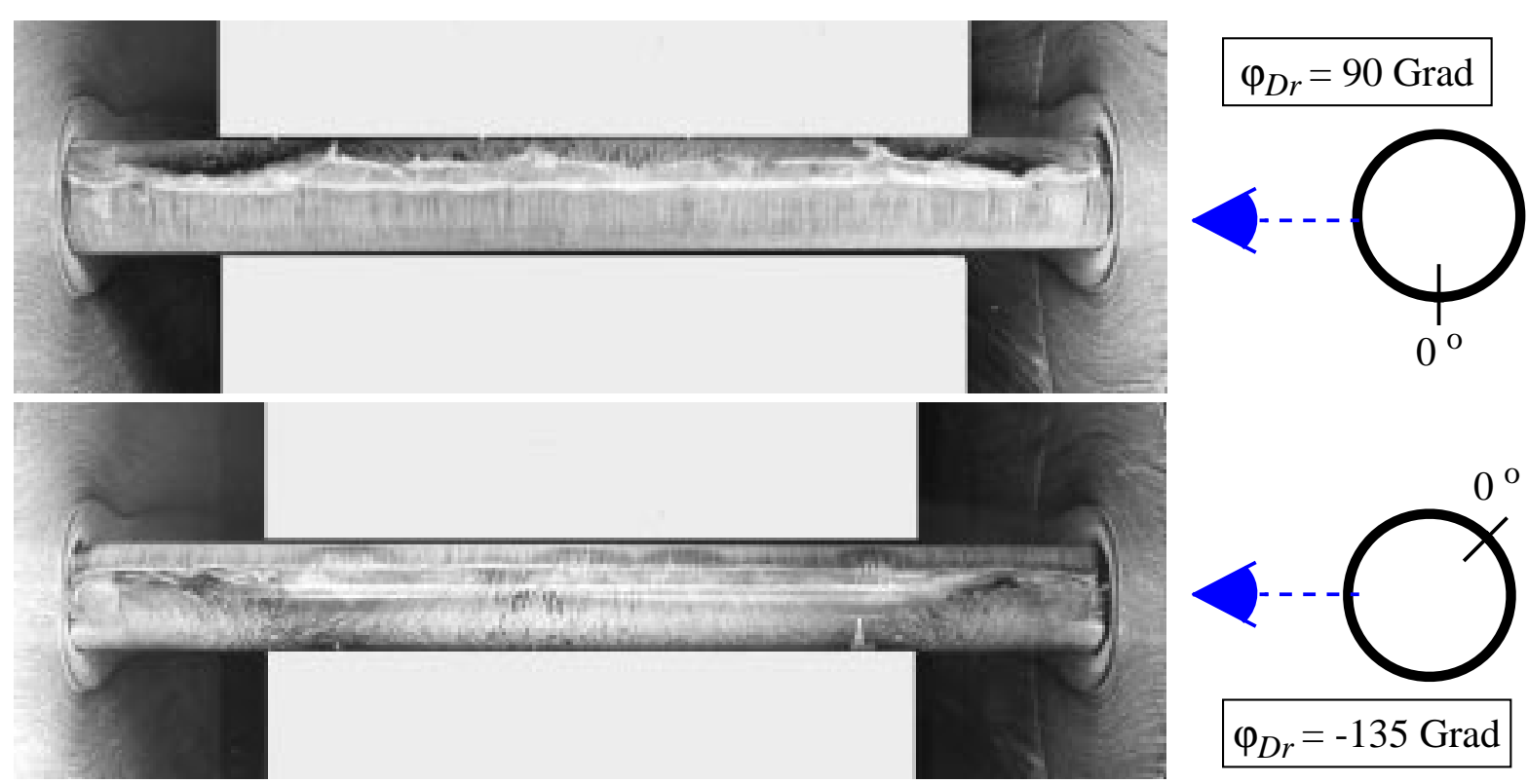

Bild 3.18 Anstrichbild bei $\operatorname{Re}_{\mathrm{D}}=1,0 \times 10^{6}$ mit einer Draufsicht unter einem Winkel von $+90 \mathrm{Grad}$ (oben) und $-135 \mathrm{Grad}$ (unten). Auf Ober- und Unterseite tritt von $y_{D}=-3$ bis $y_{D}=$ +3 zusätzlich eine zweite Ablöselinie auf (s.a. Bild A2.11).
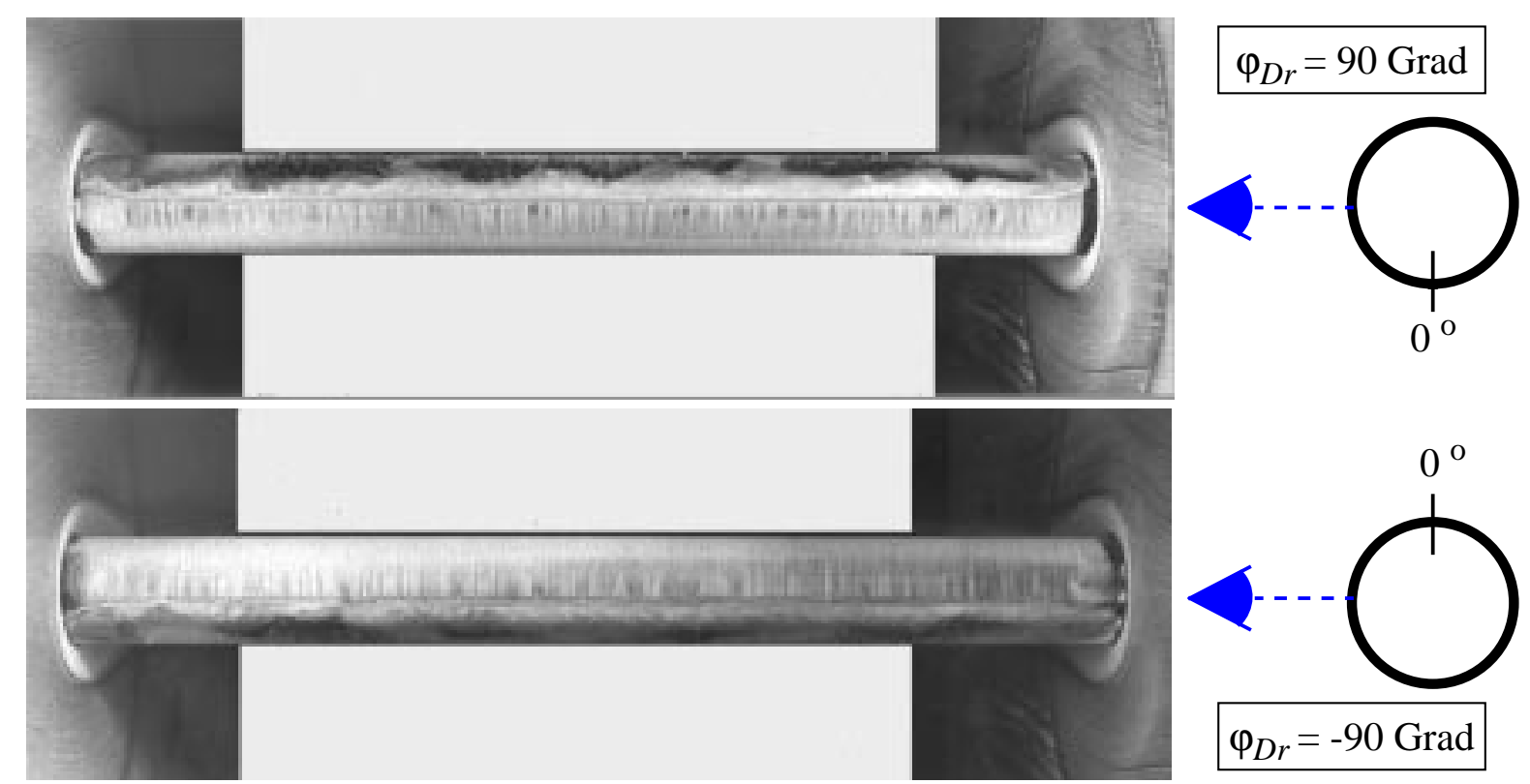

Bild 3.19 Anstrichbild bei $R e_{D}=1,6^{\times} 10^{6}$ mit einer Draufsicht unter einem Winkel von +90 Grad (oben) und -90 Grad (unten). Auf Ober- und Unterseite ist jeweils eine Ablöselinie homogen über der Spannweite ausgebildet (s.a. Bild A2.12). 
Andererseits besitzt die erste Ablöselinie, über der Spannweite einen nahezu geradlinigen Verlauf, so dass eine spannweitig homogene, also zweidimensionale Ablösung dominieren muss. Der Farbvortrag könnte demnach nicht zu diesem Strömungszustand gehören und hat sich vermutlich beim Anfahren des Windkanals ausgebildet.Die Änderung in der Ablösestruktur wie sie in den Anstrichbildern von der gemessenen Reynoldszahl von $\operatorname{Re}_{D}=1,0^{\times} 10^{6}$ bis $R e_{D}=3,0^{\times} 10^{6}$ festzustellen ist, bewirkt ein Stromaufwandern der zeitlich gemittelten Ablösung. Während in dem Anstrichbild bei $R e_{D}=1,0^{\times} 10^{6}$ die Abösung bei einem Azimutwinkel von etwa 95 Grad vermutet werden kann, beträgt sie bei der gemessenen Reynoldszahl von $\operatorname{Re}_{D}=3,0^{\times} 10^{6}$ etwa 88 Grad. Damit ist auch eine Erhöhung des Widerstandsbeiwertes von $C_{W}=0,60$ auf $C_{W}=0,90$ vernüpft. Das bedeutet, dass durch das Stromaufwandern der Ablösung das zeitlich gemittelte Rezirkulationsgebiet breiter werden muss, was zu einem größerem Saugdruck führt. Derartig große Widerstandsbeiwerte werden oberhalb des überkritischen Bereiches am glatten Zylinder nicht beobachtet, so dass die durch die Anstrichfarbe zusätzlich aufgebrachte Oberflächenrauhigkeit die Entwicklung der Grenzschicht beeinträchtigt wird (Achenbach und Heinecke 1981 [2], Zdravkovich 1990 [99]). Die Grenzschicht wird vorzeitig turbulent und löst mit zunehmender Reynoldszahl bei einem kleineren Azimutwinkel oder unter einem größeren tangentialen Winkel ab. Mit dem Übergang zum transkritischen Bereich vollzieht sich eine Umordnung der abgelösten Zylinderströmung. Während zuvor eine spannweitig periodische zellartige Längswirbelablösung stattfand, ist mit Beginn des transkritischen Bereiches wieder eine mehr spannweitig homogene Ablösung von Querwirbeln anzunehmen.
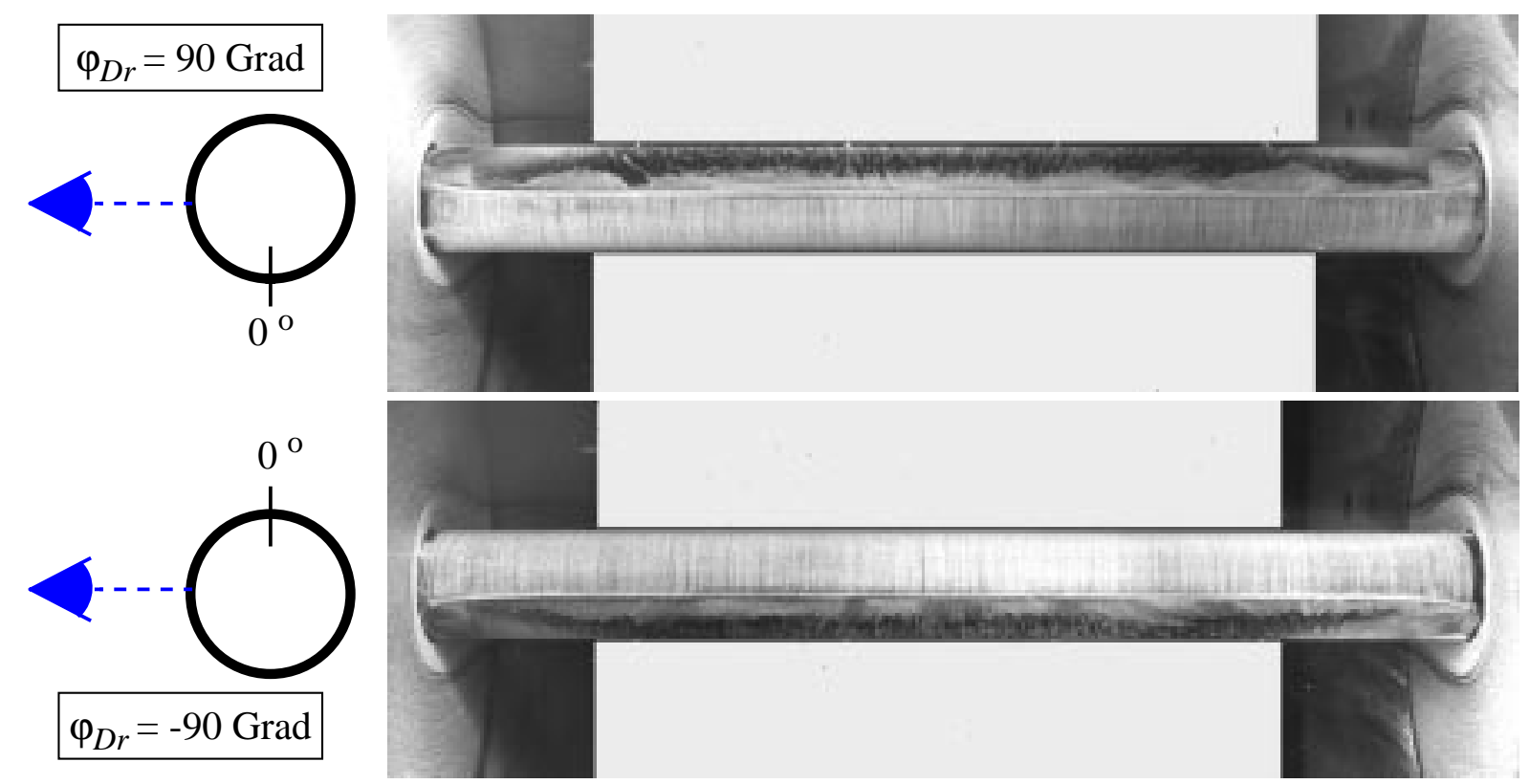

Bild 3.20 Anstrichbild bei $R e_{D}=3,0 \times 10^{6}$ mit einer Draufsicht unter einem Winkel von $+90 \mathrm{Grad}$ (oben) und -90 Grad (unten). Auf Ober- und Unterseite ist jeweils eine Ablöselinie homogen über der Spannweite ausgebildet (s.a. Bild A2.13).

Dabei könnte aus topologischen Betrachtungen heraus in Analogie zum unterkritischen Bereich ein Zwischenzustand benötigt werden, der die Ablösestruktur der doppelten Ablöseblase auf der Ober- und Unterseite des Zylinders besitzt. Mit dieser Ablösestruktur wird also wieder ein Strömungszustand erreicht, bei dem längs der Spannweite kohärente Querwirbel, ähnlich der von- 
Kármán-Wirbel im unterkritischen Bereich, ablösen. Mit noch weiterer Erhöhung der Reynoldszahl bildet sich letztendlich die bekannte Ablösestruktur mit nur einer Ablöselinie auf jeder Seite des Zylinders aus.

\section{Zeitlich gemittelte Druckmessungen}

Um die aus den Anstrichbildern ermittelten spannweitigen Wellenlängen zu bestätigen, wurden spannweitige Druckverteilungen im überkritischen und oberen Übergangsbereich am ,Aktiv“Zylindermodell aufgenommen. Für die Bestimmung der spannweitigen Wellenlänge werden die längs der Spannweite auftretenden Druckmaxima gezählt. Sie entsprechen spannweitig lokal auftretenden „Durchbrüchen“ der transitionellen Ablöseblase in den Anstrichbildern Bild $\underline{\text { 3.15}}$, Bild 3.16 und Bild 3.17.
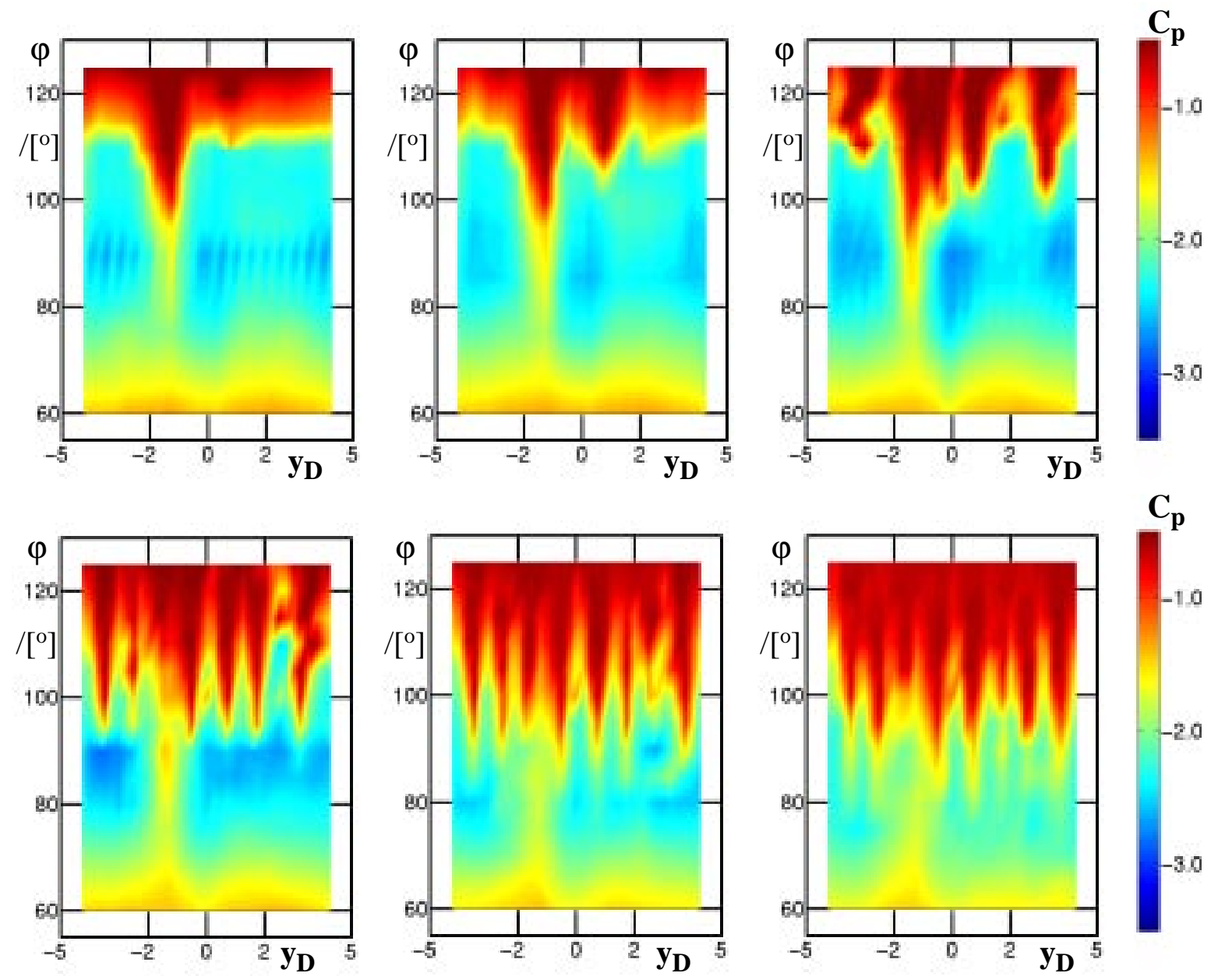

Bild 3.21 Spannweitige Druckverteilungen im azimutalen Bereich von 60 bis 125 Grad erhalten durch Drehen des Zylinders liefern mit zunehmender Reynoldszahl eine abnehmende spannweitige Wellenlänge - von links nach rechts - obere Reihe: $y_{L}=6 D$ bei $R e_{D}=0,36^{\times} 10^{6}, y_{L}=2,5 D$ bei $R_{D}=0,42^{\times} 10^{6}, y_{L}=2,0 D$ bei $R e_{D}=0,55^{\times} 10^{6}$ - untere Reihe: $y_{L}=1,7 D$ bei $R e_{D}=0,80^{\times} 10^{6}, y_{L}=1,2 D$ bei $R e_{D}=1,15^{\times} 10^{6}, y_{L}=1,2 D$ bei $\operatorname{Re}_{D}=1,80 \times 10^{6}$.

Aus Bild 3.21 wird deutlich, dass mit zunehmender Reynoldszahl die mittlere spannweitige Wellenlänge abnimmt. Zu Beginn des oberen Übergangsbereiches (hier beim „Aktiv“-Zylindermodell bei einer Reynoldszahl von $R e_{D}=0,60 \times 10^{6}$ ) beträgt die spannweitige Länge $y_{L}=1,9 D$ 
und zum Ende des oberen Übergangsbereiches nur noch $y_{L}=1,2 D$. Damit wird eine gute Übereinstimmung mit den aus den Anstrichbildern ermittelten mittleren Längen einer zellulären Ablösestruktur erreicht. Lediglich die jeweiligen Reynoldszahlen stimmen nicht überein, was wiederum auf die Oberflächenbeschaffenheit der beiden Zylindermodelle zurückgeführt werden muss. Beim „Passiv“-Zylindermodell wird mit Verlassen des kritischen Bereiches der überkritische Zustand sofort instabil. Spannweitig periodische Ablösestrukturen mit einer Wellenlänge von etwa $y_{L}=2 D$ bilden sich aus. Beim ,Aktiv“-Zylindermodell sind es die Bohrungen für die Druckmessung, die die Grenzschichtentwicklung stören, wenn auch nicht in dem Maße wie die Anstrichfarbe. Die periodische Ablösestruktur mit einer spannweitigen Wellenlänge von $y_{L}=2 D$ wird hier erst bei einer Reynoldszahl von $R e_{D}=0,60 \times 10^{6}$ beobachtet. Weiterhin, wie bereits im kritischen Bereich festgestellt werden musste, wird die Ausbildung des azimutalen Druckmimums bei einer Spannweite von etwa $y_{D}=-1,5$ behindert. Dennoch ist anhand von Bild 3.21 abzulesen, dass mit steigender Reynoldszahl die Wirkung der Störung schwächer und die spannweitig periodische Entstehung von Längswirbeln ab einer Reynoldszahl von etwa $R e_{D}=0,80^{\times} 10^{6}$ nicht mehr wesentlich beeinträchtigt wird.

Während im unterkritischen bis überkritischen Bereich Querwirbel-Ablösezustände zu den Strouhalzahlen , $, S r=0,2^{\prime \prime},{ }_{,}, \mathrm{Sr}=0.3$ “ und $, S r=0,5^{\text {" }}$ das instationäre Verhalten bestimmen, ist mit der Längswirbel dominierten Ablösung im oberen Übergangsbereich ein anderes instationären Verhalten zu erwarten. Im transkritischen Bereich hingegen müsste sich wieder das charakteristische instationäre Bild der Querwirbel dominierten Ablösung zeigen.

\section{Instationäre Kraftmessungen}

Für die Untersuchung des instationären Ablöseverhaltens wurde wieder das ,Referenz“-Zylindermodell genutzt. Aus dem Verhalten des Widerstandsbeiwertes und des Effektivwertes der Auftriebsschwankungen (Bild 3.1) konnte bereits geschlossen werden, dass mindestens zwei Übergänge im oberen Übergangsbereich stattfinden müssen. Der eine Übergang, im folgenden

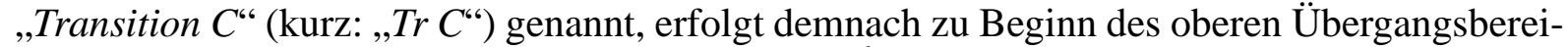
ches bei einer Reynoldszahl um etwa $R e_{D}=1,0 \times 10^{6}$, in dem ein Anwachsen des Widerstandsbeiwertes und der Schwankungen des Auftriebsbeiwertes und eine sprunghafte Änderung in der Strouhalzahl der dominanten Wirbelablösefrequenz zu verzeichnen ist (Bild 3.1) . Ein weiterer Übergang, im folgenden „Transition $D^{“}$ (kurz: „Tr $D^{“}$ ) genannt, ist mit dem lokalen Maximum im Effektivwert der Auftriebsschwankungen bei einer Reynoldszahl um etwa $\operatorname{Re}_{D}=2,1 \times 10^{6}$ verbunden.

In Bild 3.22 sind die Auftriebsschwankungen für die jeweiligen Strouhalzahlen bzw. Ablösemoden und die zugehörigen Spektren in Abhängigkeit von der Reynoldszahl für den Beginn des oberen Übergangsbereiches aufgetragen. Während für Reynoldszahlen unterhalb von etwa $R e_{D}$ $=1,2^{\times} 10^{6}$ die Strouhalzahl der dominanten Wirbelablösefrequenz etwa $\mathrm{Sr}=0,5$ beträgt, ist es oberhalb davon die Strouhalzahl mit $S r=0,1$. Daraus kann entnommen werden, dass nun die periodische Ablösung nicht mehr von Querwirbeln, also von-Kármán-ähnlichen Wirbeln bestimmt wird. In den dazugehörigen Spektren ist ersichtlich, dass dieser Übergang in Analogie zu den kritischen Übergängen ebenfalls in drei Schritten abläuft. Zunächst nehmen die periodischen Schwankungen bei $S r=0,5 \mathrm{ab}$. Dies erfolgt solange bis die Schwankungen bei $S r=0,1$ und bei $S r=0,5$ etwa die gleiche Amplitude besitzen. Damit stehen diese beiden instationären Zustände in Konkurrenz zueinander, was zusätzlich mit niederfrequenten Schwankungen, sog. kritischen Fluktuationen, unterhalb von $S r<0,1$ verbunden ist. Mit Verlassen dieses kritischen, 
instabilen Zustandes erfolgt mit zunehmender Reynoldszahl das Anwachsen der Schwankungen zu der Strouhalzahl $S r=0,1$ und dominieren das instationäre Ablöseverhalten.

Die spektrale Verteilung im Amplitudenspektrum um $S r=0,1$ ist sehr breitbandig und erstreckt sich über einer relativ großen Strouhalzahlbereich von $S r=0,05$ bis etwa $S r=0,20$, also $\Delta S r=$ 0,15. Dies ist ein Ausdruck für eine mehr oder weniger spannweitig inhomogene Ablösung der Zylinderströmung. Während im überkritischen Bereich die Breite der schmalbandigen Verteilung bei etwa $S r=0,5$ nur $\Delta S r=0,05$ beträgt und somit von einer deutlich ausgeprägten spannweitig kohärenten Querwirbelablösung ausgegangen werden kann. Anhand der zuvor gezeigten Anstrichbilder als auch der spannweitigen Druckverteilungen für diesen Reynoldszahlbereich können die Schwankungen im Auftrieb bei $S r=0,1$ somit den spannweitig periodisch abgelösten Längswirbelstrukturen zugeordnet werden.
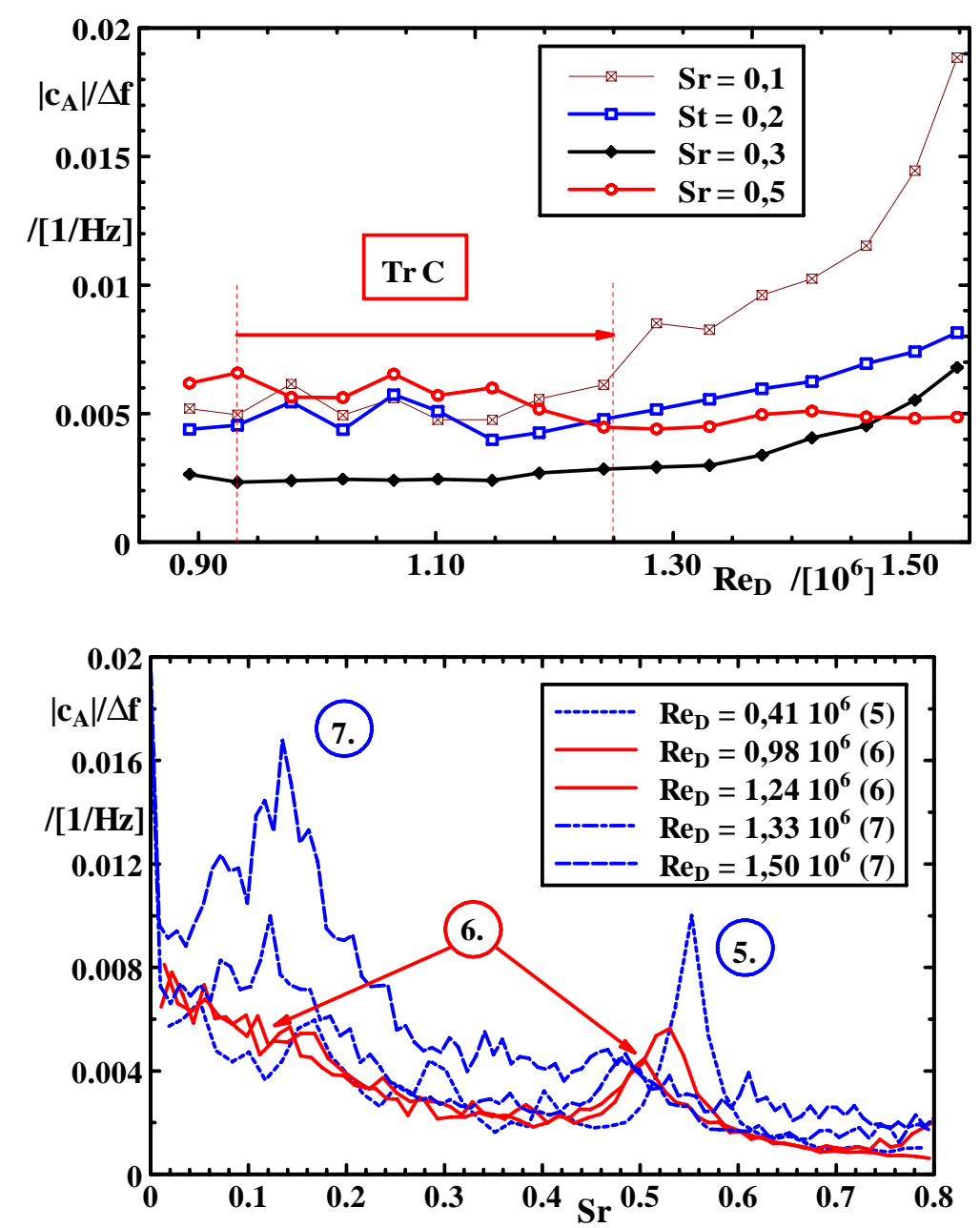

Bild 3.22 Amplituden für bestimmte Strouhalzahlen (oben) und zugehörige Spektren der Auftriebsschwankungen zu Anfang des oberen Übergangsbereiches (unten). (5) überkritischer Zustand mit Ablösung von Querwirbeln, (6) konkurrierender Zustand zwischen Quer- und Längswirbelablösung, (7) Längswirbel dominierte Ablösung.

Mit dem bei der Reynoldszahl $R e_{D}=0,41 \times 10^{6}$ hergestellten Anstrichbild (Bild 3.15) lässt sich die topologische Struktur einer Zelle mit einem ablösenden Längswirbelpaar nachzeichnen 
(Bild 3.23). An der spannweitigen Stelle, wo es zum „Durchbruch“ der Ablöseblase kommt, lösen lokal Querwirbel ab. Diese Querwirbel werden aber in ihrer Stromabentwicklung von den spannweitig benachbarten Unterdruckgebieten abgelenkt. Das mit jeder Zelle ablösende Längswirbelpaar verwirbelt entsprechend mit diesen Querwirbeln. Die Folge ist die Ausbildung einer spannweitig periodischen Ablösestruktur von Längswirbelpaaren, die im oberen Übergangsbereich das zeitlich gemittelte wie das instationäre Ablöseverhalten bestimmen.

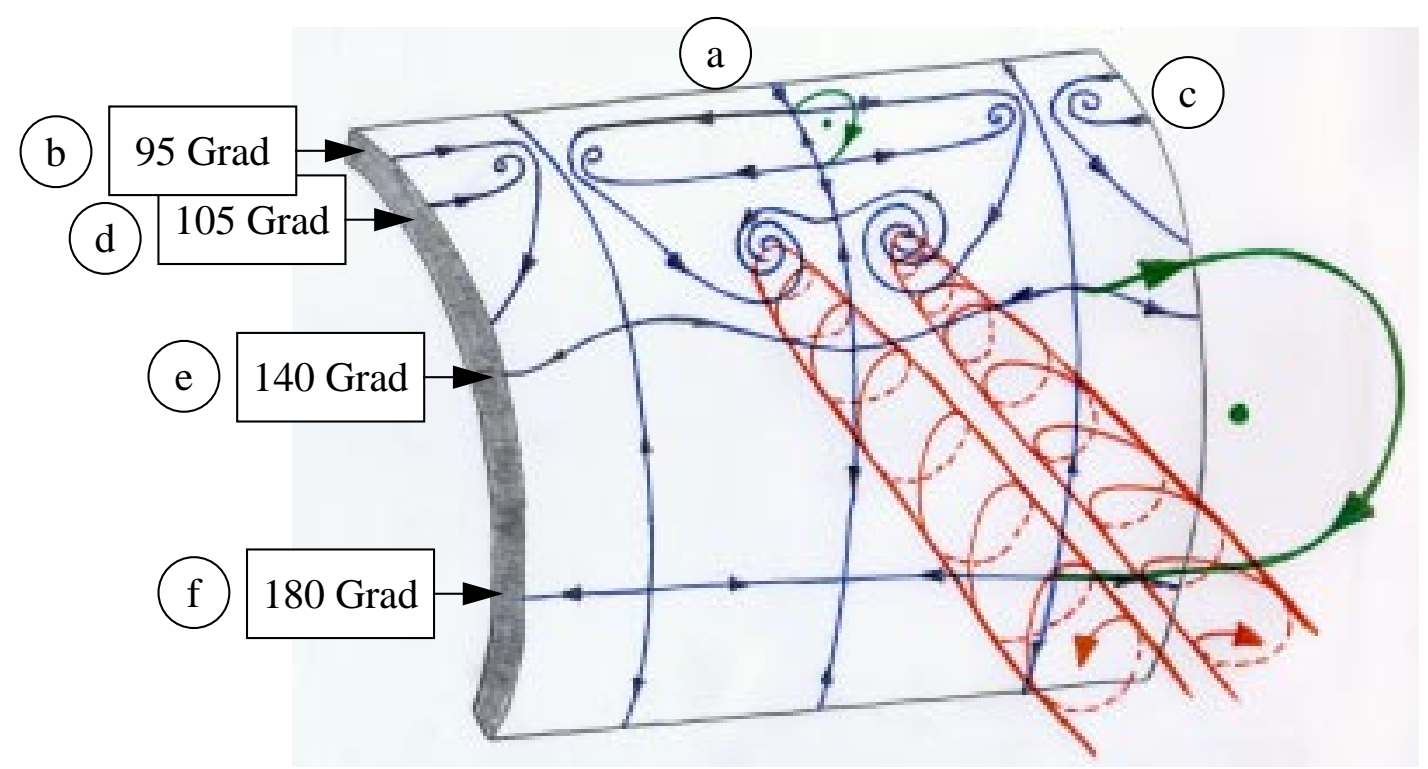

Bild 3.23 Vereinfachte schematische Darstellung der Ablösetruktur einer Zelle der spannweitig periodischen abgelösten Zylinderströmung entsprechend des Anstrichbildes Bild 3.15: (a) anliegende laminare Grenzschicht; (b) laminare Ablöse-"Linie"; (c) spannweitig instabile transitionelle Ablöseblase; (d) Wiederanlege-"Linie“; (e) turbulente Ablöse-"Linie“" und (f) leeseitig abgelöster Strömungsbereich mit zeitlich gemitteltem Wiederanlegen im hinteren Staupunktbereich.

Wie bereits aus den Anstrichbildern Bild 3.17 und Bild 3.18 hervorgeht und diskutiert wurde, ist aber auch dieser Strömungszustand nicht stabil. Zunächst wächst mit weiter zunehmender Reynoldszahl die Amplitude der periodischen Schwankungen bei $\mathrm{Sr}=0,1$ weiter an und erreicht bei einer Reynoldszahl von etwa $R e_{D}=2,0^{\times} 10^{6}$ ein lokales Maximum (Bild 3.24). Mit weiter zunehmender Reynoldszahl jenseits dieser Reynoldszahl fällt der Effektivwert der Auftriebsbeiwertschwankungen als auch die Betragsamplitude bei $S r=0,1$ drastisch ab. Bei einer Reynoldszahl von etwa $R e_{D}=2,3^{\times} 10^{6}$ wird auch hier wieder ein konkurrierender Zustand erreicht. Diesmal tragen Längs- wie Querwirbelablösungen in gleichem Maße zur Dynamik bei. Wird die Reynoldszahl über diesen konkurrierenden Zustand hinaus erhöht, wächst die Betragsamplitude zu , $S r=0,2$ “ weiter an, während die Betragsamplitude bei der Strouhalzahl zu ,Sr=0,1“ entschieden weiter abfällt. Die Breite des Peaks bei $S r=0,2$ mit $\Delta S r=0,07$ erweist sich damit wieder als schmalbandig, so dass in Übereinstimmung mit Ergebnissen von Schewe (1983) [75] und Roshko (1961) [71] der transkritische Bereich erreicht ist. Der Ort der laminar-turbulenten Transition ist erstmalig stromauf der Ablösung, so dass nunmehr die turbulente Grenzschicht spannweitig homogen vom Zylinder periodisch ablöst.

In Analogie zum (unteren) kritischen Übergangsbereich könnte auch im oberen (kritischen) Übergangsbereich Hysterese vermutet werden. Die Änderungen in den Beiwerten sind aber hier 
zu gering, um daran die Hysterese zu beobachten. Vielmehr muss das spannweitige Ablöseverhalten betrachtet werden. Es wäre denkbar, dass die Übergänge von einer Längswirbelstruktur zu einer anderen Längswirbelstruktur mit einer anderen spannweitigen Wellenlänge der Hysterese unterliegt.
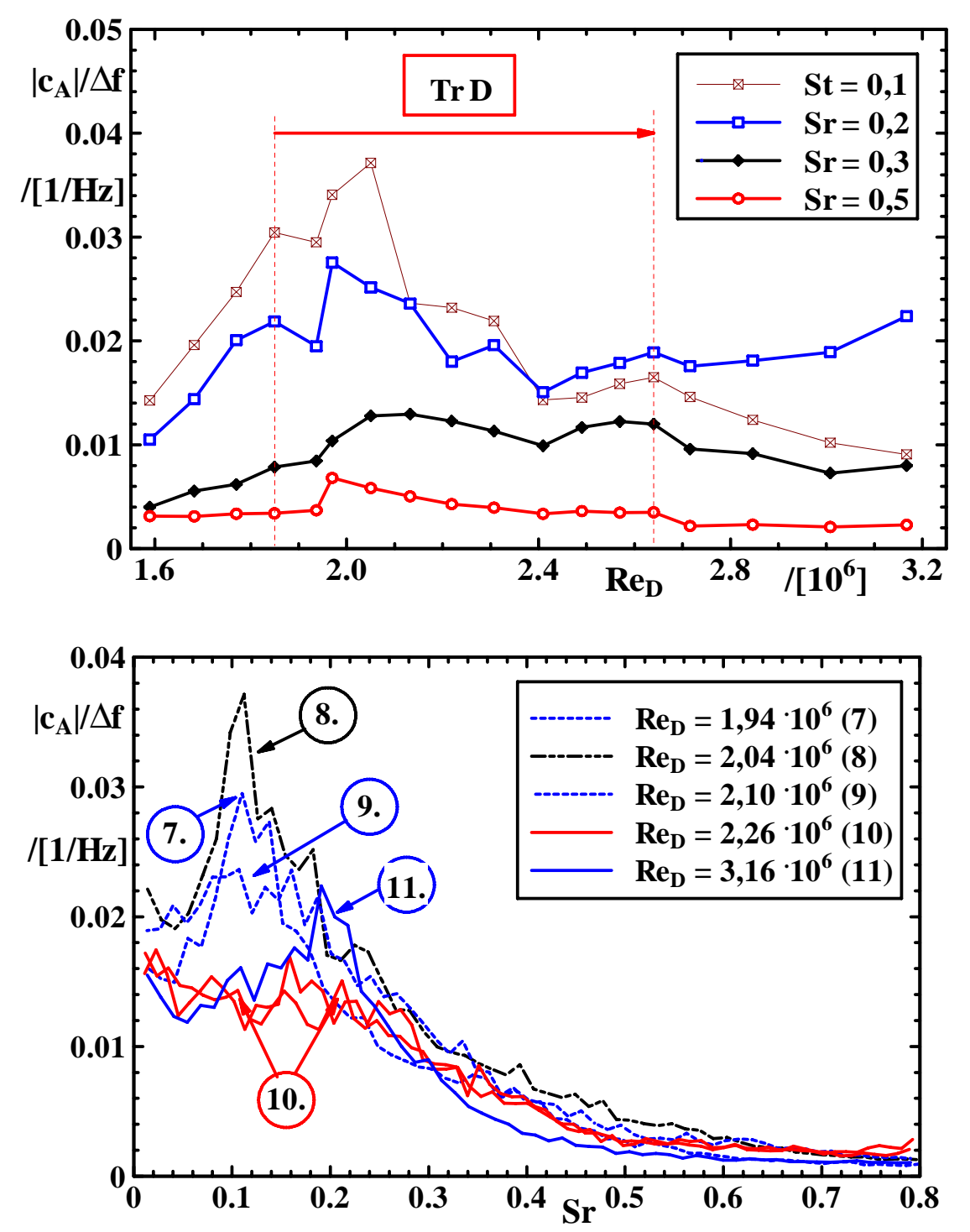

Bild 3.24 Amplituden der Auftriebsschwankungen für bestimmte Strouhalzahlen (oben) und zugehörige Spektren im oberen Übergangsbereich (unten): (7) Längswirbel dominierte Ablösung, (8) kritische instationäre Schwankungen durch Wechselwirkung von Längs- und Querwirbeln, (9) instabiler Zustand der Längswirbelablösung, (10) konkurrierender Zustand zwischen Längs- und Querwirbelablösung, (11) Querwirbel dominierte Ablösung. 


\subsection{Der Zusammenhang zwischen Widerstands- und Basisdruckbeiwert}

Bereits früh wurde erkannt (von Kármán 1911 [93]), dass der negative Basisdruckbeiwert, also die Saugwirkung hinter einem stumpfen Körper - wie dem querangeströmten Kreiszylinder maßgebend für die Größe des Widerstandes ist, zumal in dem Transitions-Reynoldszahlbereich für Reynoldszahlen größer als $\operatorname{Re}_{\mathrm{D}}>10^{5}$ der Druckwiderstand den Gesamtwiderstand fast ausschließlich bestimmt und der Reibungswiderstand vernachlässigt werden kann (Achenbach 1981 [2]).

Auch für die Beschreibung der Strömungszustände in Abhängigkeit von der Reynoldszahl benutzen neben dem Widerstandsbeiwert (Achenbach 1979 [1], Schewe 1983 [75]) auch einige Autoren den negativen Basisdruckbeiwert, auch Basissaugbeiwert genannt (,suction coefficient", Roshko 1993 [70], Williamson 1996 [96]). Tatsächlich stimmen der Widerstandsbeiwert und der negative Basisdruckbeiwert mindestens für Reynoldszahlen größer als $R e_{D}>10^{4}$ gut überein. Begründet wird diese Eigenschaft auf der Grundlage geometrischer Überlegungen nach Roshko (1954) [70] und neuerdings nach Steinhoff (1999) [84].

Weiterhin zeigte Bearman (1969) [7], dass die spannweitige Basisdruckverteilung für verschiedene Reynoldszahlen beim laminar-turbulenten Übergang nahezu zweidimensional ist, wenn von Randeffekten abgesehen wird. Die Wirkung sog. Hufeisenwirbel, die bei der Nutzung von Seitenendscheiben oder einer geschlossenen Messstrecke sich ausbilden, beeinträchtigen das statische Druckfeld an den seitlichen Begrenzungen des Zylinders bis zu einer Tiefe von etwa $1 D$.

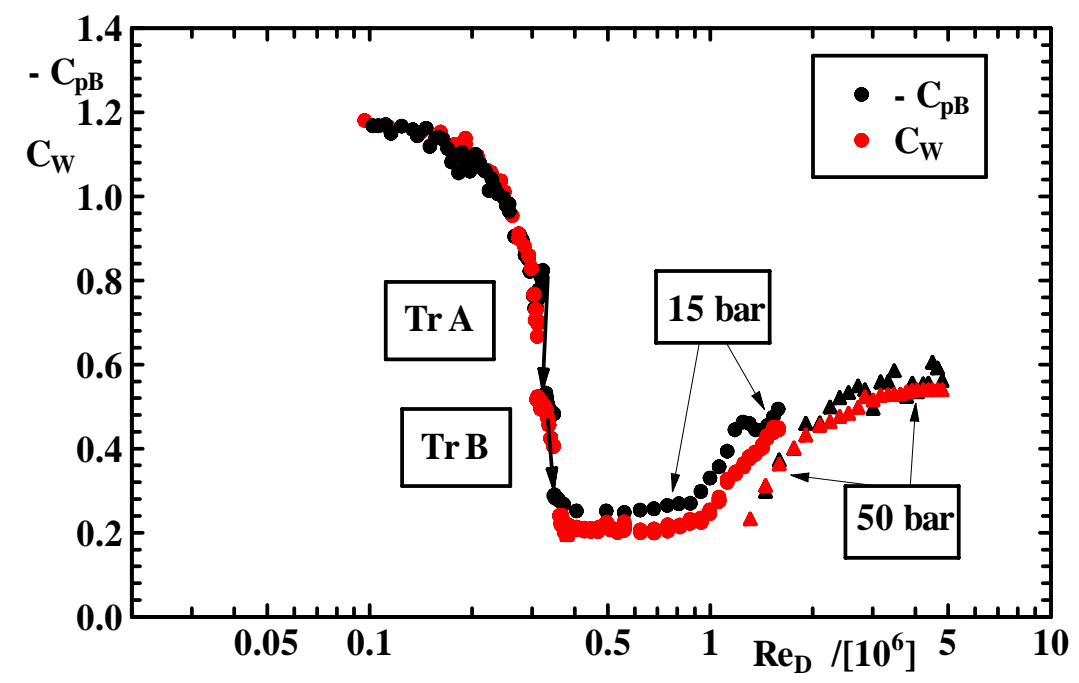

Bild 3.25 Vergleich von Widerstandsbeiwert und negativem Basisdruckbeiwert in Abhängigkeit von der Reynoldszahl beim laminar-turbulenten Übergang der ablösenden Zylindergrenzschicht

Um den Zusammenhang zwischen Widerstandsbeiwert und Basisdruckbeiwert für Aussagen zur Widerstandsreduktion bei der Anwendung von aktiven Beeinflussungsverfahren, die spannweitig differenziert die abgelöste Strömung einseitig beeinflussen sollen, benutzen zu können, wurde die spannweitige Basisdruckverteilung mit einer Auflösung von $\Delta \varphi_{P}=0,5 D$ gemessen. Dazu wurden simultan die Kräfte zur Bestimmung des Widerstandsbeiwertes erfasst. Die beiden Kurven sind in Bild 3.25 dargestellt, wobei zum Vergleich der negative Basisdruckbeiwert 
bei der Spannweite $y_{D}=0,0$ benutzt wurde.

Zunächst kann gesagt werden, dass im allgemeinen eine sehr gute Übereinstimmung zwischen negativem Basisidruckbeiwert und Widerstandsbeiwert vorliegt. Geringe Abweichungen sind allerdings im kritischen Bereich im asymmetrisch abgelösten Zustand und teilweise im oberen Übergangsbereich festzustellen.
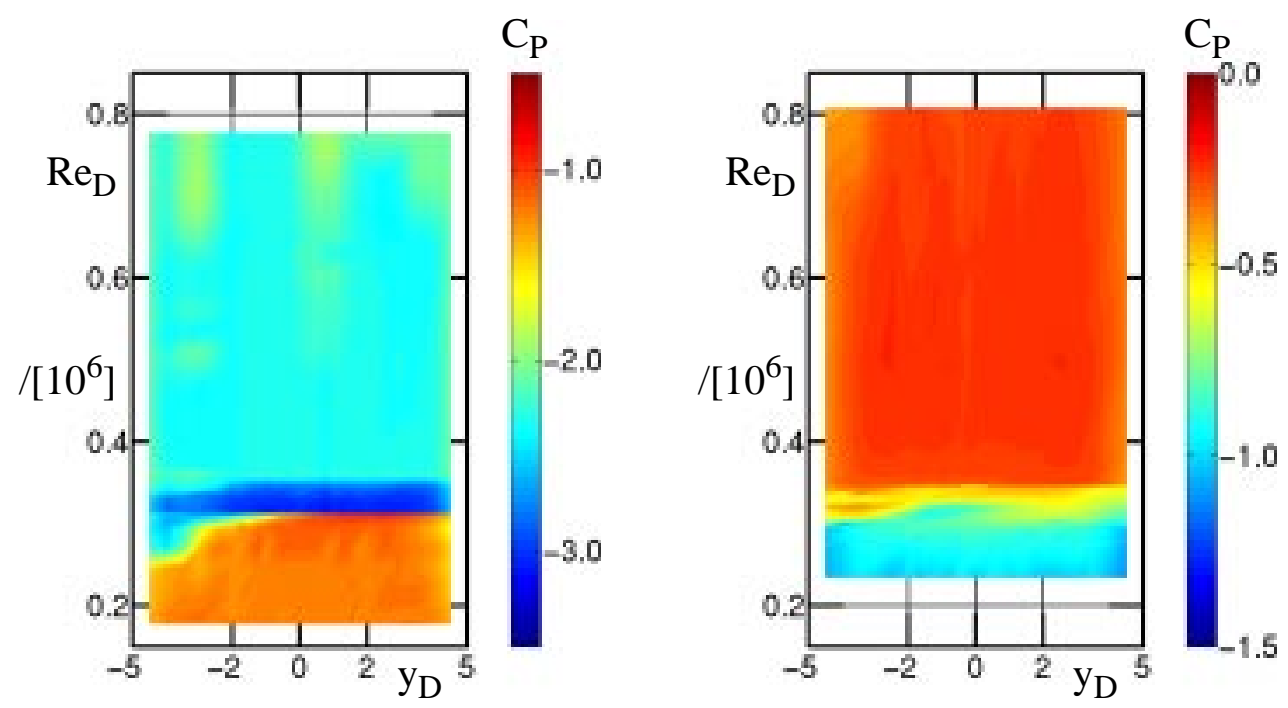

Bild 3.26 Spannweitige Verteilungen des statischen Druckbeiwertes bei einem Azimutwinkel von $\varphi_{P}=70 \mathrm{Grad}$ (links) und von $\varphi_{P}=180 \mathrm{Grad}$ (rechts) in Abhängigkeit von der Reynoldszahl im Bereich von $\operatorname{Re}_{D}=0,2^{\times} 10^{6}$ bis $\operatorname{Re}_{D}=0,8^{\times} 10^{6}$.

Die Ursache für die Abweichung im kritischen Bereich kann anhand Bild 3.26 links abgelesen werden. Die „Transition A“ erfolgt nicht spannweitig homogen. Die spannweitige Druckverteilung deutet auf eine Störung der ablöesenden Grenzschicht, die von der Innenseitenwand der Messstrecke herrührt. Die Ursache dafür konnte nicht geklärt werden. Diese Störung wirkt sich allerdings auch auf die spannweitige Verteilung des Basisdruckbeiwertes aus. In diesem Reynoldszahlausschnitt, wie in Bild 3.26 rechts zu sehen, erreicht der Bassidruckbeiwert nur annährend ein konstanten Wert längs der Spannweite .

Im oberen Übergangsbereich beeinträchtigen spannweitig periodische Ablösestrukturen den homogenen spannweitigen Basisdruckbeiwertverlauf. Insbesondere bei einer Reynoldszahl von etwa $R e_{D}=1,3^{\times} 10^{6}$ tritt unerwartet ein geringer Anstieg im Basisdruckbeiwert (s. Bild 3.25) auf. Da sich im oberen Übergangsbereich spannweitig periodische Wirbelstrukturen mit unterschiedlicher spannweitiger Wellenlänge ausbilden, könnte sich bei dieser Reynoldszahl der Übergang von einer Ablösestruktur zu einer anderen mit kleinerer spannweitiger Wellenlänge vollziehen. In Bild 3.26 links ist die Ausbildung spannweitig periodischer Wirbelstrukturen ab einer Reynoldszahl von $\operatorname{Re}_{D}=0,7 \times 10^{6}$ zu erkennen. Der spannweite Verlauf des Basisdruckbeiwertes hingegen erweist sich in dem überkritischen Bereich als nahezu konstant (Bild 3.26 rechts).

Um Fehlinterpretationen des Basisdruckbeiwertes zu vermeiden, wird im folgenden über mehrere spannweitige Positionen gemittelt. Somit kann bei Anwendung der aktiven Beeinflussungsverfahren die Änderung des spannweitig gemittelten Basisdruckbeiwertes, im folgenden einfach Basisdruckbeiwert, als Maß für Änderungen im Widerstandsbeiwert benutzt werden: 


$$
\Delta C_{W} \approx\left(-\Delta C_{p B}\right) .
$$

Darüberhinaus könnte auch aus der azimutalen Druckverteilung der Druckwiderstands- und Auftriebsbeiwert ermittelt werden. Allerdings wäre der hierbei gemachte Fehler größer, da beim „Aktiv“-Zylindermodell nur bei einer spannweitigen Position $y_{D}=0$ eine ausreichend hohe Anzahl an Druckmessstellen vorhanden ist.

\subsection{Aussagen für die Anwendung der Beeinflussungsverfahren}

Mit den vorliegenden Ergebnissen wird im folgenden versucht für den laminar-turbulenten Übergang der natürlich abgelösten Zylindergrenzschicht ein vereinfachendes Instabilitätsmodell zu konstruieren. Die vielfältigen räumlichen und zeitlichen Änderungen im Ablöseverhalten könnten auf der Wirkung und der Wechselwirkung von globalen Instabilitäten der Zylinderströmung bzw. konvektiver Instabilitäten der Grenzschicht und der freien abgelösten Scherschichten beruhen. Globale Instabilitäten bestimmen dabei maßgeblich das instationäre Verhalten, während konvektive Instabilitäten zu Änderungen des räumlichen Ablöseverhaltens führen. Das instationäre Ablöseverhalten ist dabei Grundlage für eine Wechselwirkung der Instabilitäten. Darüber hinausgehend könnte festgestellt werden, dass eine zeitlich gemittelte, instationäre insbesondere turbulente Strömung ein global instabiles Verhalten zeigen kann, welches nicht aus der Wirkung lokaler Instabilitätsformen allein folgen muss. Dabei stellt sich jedoch das theoretische Verifikationsproblem der Identifikation und Beschreibung der eigentlich global instabil werdenden Grundströmung.

\section{Zeitlich periodische Ablösung von Wirbelstrukturen beim laminar-turbulenten Übergang}

Die Änderung der Ablösestruktur im unterkritischen Bereich von einer Ablöselinie zur doppelten Ablöseblase ermöglicht erst den Widerstandsabfall mit Beginn des kritischen Bereiches ab $R e_{D}=2,0^{\times} 10^{5}$. Der Winkel, unter dem tangential die Grenzschicht vom Zylinder ablöst, kann sich verringern, ohne dass der Azimutwinkel der Ablösung sich signifikant ändern muss. Damit folgt eine Verringerung der Nachlaufbreite und somit eine Anhebung des Basisdruckes bzw. Minderung des Widerstandes. Gleichzeitig ist aus den Anstrichbildern ablesbar, dass die Azimutposition der zeitlich gemittelten Ablösung beginnend im unterkritischen Bereich bis kurz vor der „Transition $A$ “ bis zu einer Reynoldszahl von etwa $R e_{D}=3,1 \times 10^{5}$ sich nur unmerklich verändert hat. Damit verbunden ist auch eine nahezu konstante Strouhalzahl der Wirbelablösung mit $S r=0,20$, während der Widerstandsbeiwert bereits auf $C_{W}=0,8$ abgefallen ist. Erst mit noch weiter zunehmender Reynoldszahl fällt die Strouhalzahl bis auf einen Wert von $\mathrm{Sr}=$ 0,16 und der Widerstandsbeiwert auf $C_{W}=0,7 \mathrm{ab}$. Nach der klassischen Beziehung (Roshko 1954 [70]) zwischen Widerstandsbeiwert und Strouhalzahl, gegeben zu

$$
\left.C_{W} \sim \frac{b}{D} \sim \frac{1}{S r} \rightarrow \frac{\lambda_{K W}}{D}\right|_{W \rightarrow 0},
$$

wobei $b$ die Nachlaufbreite, $\lambda_{K W}$ den kleinsten Abstand gleichsinnig rotierender von-KármánWirbel und $W$ hier die zeitlich gemittelte Geschwindigkeit normal zur Strömungsrichtung bezeichnet, müsste mit der Abnahme des Widerstandsbeiwertes die Strouhalzahl zunehmen. Diese Beziehung hat sicherlich seine Gültigkeit für Reynoldszahlen bis etwa $R e_{D} \approx 5000$, in dem der 
Reibungswiderstand noch maßgeblich zum Gesamtwiderstand beiträgt (s. Achenbach 1979 [1]). Andererseits könnte das Stromaufwandern der Transition in den freien abgelösten Scherschichten für das „veränderte“ Ablöseverhalten verantwortlich sein. Wenn dass so ist, muss der Entstehungsmechanismus der Querwirbel im Rezirkulationsgebiet ein anderer sein als bei laminarem Nachlauf und laminaren abgelösten Scherschichten. D.h., die globale Instabilität der Grundströmung müsste durch eine globale Instabilität, die auf der Wechselwirkung der instabilen abgelösten Scherschichten beruht, ersetzt werden. Es müsste eine Kennzahl existieren, die bei Querwirbelablösung am Zylinder im unterkritischen bis überkritischen Bereich einen konstanten Wert annimmt. Dass dies möglich scheint, ergibt sich aus der Darstellung der Strouhalzahlen nach dem sog. „Goldenen Schnitt“ (Schewe 1985 [76]). Bekanntermaßen bilden in der Natur auftretende, fraktale Strukturen bestimmte Längenverhältnisse aus, die dem „Goldenen Schnitt" entsprechen und mit einem zumeist nichtlinearen Gleichungssytem beschrieben werden können.

Der Widerstandsbeiwert ist sicherlich proportional zur Querausdehnung des Rezirkulationsgebietes, so dass die Nachlaufbreite ins Verhältnis zum Zylinderdurchmesser gebracht werden darf. Für die Bestimmung der Strouhalzahl allerdings muss die Konvektivgeschwindigkeit der Querwirbel und eine bestimmte Länge, die die Ablöseorte der Grenzschichten mit dem Entstehungsort der Querwirbel in Beziehung setzt, herangezogen werden. Da die Konvektivgeschwindigkeit sich proportional $\mathrm{zu}$ der Anströmgeschwindigkeit verhält, kann auch die Anströmgeschwindigkeit genutzt werden. Der im Rezirkulationsgebiet entstehende Querwirbel, auch Primärwirbel genannt, muss sich zunächst vollständig ausbilden, bevor er konvektiv abschwimmen kann. Seine Entstehung kann auf einen Wechselwirkungsmechanismus zwischen den periodisch ablösenden Scherschichten von Ober- und Unterseite des Zylinders verstanden werden.

Wie phasengemittelte Messungen mit der dreidimensionalen Laser-Doppler-Anemometrie des Ablösegebietes zeigen (Brede und Leder 2001 [17]), ist mit der Bildung eines Primärwirbels ein lokales Maximum in der Reynolds'schen Schubspannung 〈 $u$ 'w' $\rangle$ oberhalb und unterhalb des Wirbelkerns des Primärwirbels zu beobachten. Die Bildung eines Primärwirbels ist mit einem Maximum in der Normalspannung normal zur Strömungsrichtung $\left\langle w^{, 2}\right\rangle$ verknüpft. Während der Primärwirbel und mit ihm das Maximum in $\left\langle w^{2}\right\rangle$ abschwimmt, wandern die Maxima von $\left\langle u^{\prime} w^{\prime}\right\rangle$ in Richtung der jeweiligen Ablöseorte auf jeder Seite des Zylinders. Erreicht das lokale Maximum in $\left\langle u\right.$ ' $\left.w^{\prime}\right\rangle$ den Ablöseort, besitzt hier auch die Normalspannung $\left\langle u^{\prime 2}\right\rangle$ seinen maximalen Wert, während er im Moment der Bildung des Primärwirbels minimal war. Damit ergibt sich ein gegenphasiges periodisch pulsierendes Bild der Schubspannungen $\left\langle w^{2}\right\rangle$ am Ort der Primärwirbelentstehung und $\langle u, 2\rangle$ am Ort der Grenzschichtablösung, wobei die Reynolds'sche Schubspannung $\left\langle u\right.$ ' $\left.w^{\prime}\right\rangle$ zwischen diesen beiden Orten als „Transporter" von Turbulenz verstanden werden kann. Damit ist die zeitlich periodische Entwicklung eines Primärwirbels abhängig von der Entfernung des Generationsortes der Primärwirbel zu den Ablöseorten der Grenzschichten. Da die gwöhnliche Strouhalzahl aber die periodische Entstehung eines Wirbelpaares beschreibt, ist die zweifache Weglänge zu nehmen. Mit Hilfe der Leitstrahlmethode kann die räumlich zeitliche Entwicklung der abgelösten Scherschicht auf den Zylinderumfang projeziert werden, so dass formal eine sog. Rückkopplungslänge $L_{f}$ beschrieben werden kann. Sie ergibt sich aus der zweifachen Bogenlänge der Differenz der Azimutwinkel der beiden Ablöseorte über den hinteren Staupunkt $\Delta \varphi_{S}$.

$$
\frac{L_{f}}{D}=\left(\left(180-\varphi_{S, \text { oben }}\right)+\left(180-\varphi_{S, \text { unten }}\right)\right) \frac{4 \pi}{360}
$$


Eine neue Strouhalzahl $S r_{f}$ wird damit gebildet zu:

$$
S r_{f}=\frac{L_{f}}{D} S r
$$

Aus Tabelle 3.1 und Tabelle 3.2 ist zu entnehmen, dass das Produkt aus Rückkopplungslänge $L_{f}$ und Strouhalzahl $S r$ für die betrachteten abgelösten Strömungszustände nahezu konstant ist und $\mathrm{zu}$

$$
S r_{f}=1,4 \pm 0,1
$$

angegeben werden kann. Ausnahmen sind lediglich für Reynoldszahlen im oberen Übergangsbereich und kurz vor der „Transition A“ festzustellen. Da im oberen Übergangsbereich das Ablöseverhalten durch Längswirbelstrukturen dominiert wird, ist auch zu erwarten, dass eine Wechselwirkung zwischen den Scherschichten in der oben beschriebenen Weise nicht erfolgt. Weiterhin könnte generell daraus geschlossen werden, dass bei signifikanten Abweichungen von dem Wert von $S r_{f}=1,4$ eine Querwirbelbildung durch Längswirbelentstehung unterdrückt wird. Mit diesem Ergebnis kann geschlussfolgert werden, dass in der Tat eine Wechselwirkung zwischen den instabilen Scherschichten und der Bildung der Querwirbel durch periodischen Transport Reynolds'scher Schubspannung existieren kann. In den Übergangsbereichen, insbesondere im oberen Übergangsbereich, muss demzufolge ein anderer Mechanismus existieren.

\begin{tabular}{|l|l|l|l|l|l|}
\hline \multicolumn{1}{|c|}{$R e_{D}$} & \multicolumn{1}{|c|}{$S r$} & \multicolumn{1}{|c|}{$\begin{array}{c}\varphi_{\text {A,oben }} \\
/[\mathrm{Grad}]\end{array}$} & $\begin{array}{c}\varphi_{\text {A, unten }} \\
/[\mathrm{Grad}]\end{array}$ & $\begin{array}{c}\Delta \varphi_{S} \\
/[\mathrm{Grad}]\end{array}$ & \multicolumn{1}{|c|}{$S r_{f}$} \\
\hline \hline $1,0 \times 10^{3}$ & 0,22 & 96 & 96 & 168 & 1,29 \\
\hline $5,0 \times 10^{3}$ & 0,21 & 87 & 87 & 186 & 1,36 \\
\hline $1,0 \times 10^{4}$ & 0,21 & 84 & 84 & 192 & 1,41 \\
\hline $5,0 \times 10^{4}$ & 0,20 & 82 & 82 & 196 & 1,37 \\
\hline
\end{tabular}

Tabelle 3.1 Berechnung der Strouhalzahl $S r_{f}$ von $R e_{D}=5,0 \times 10^{3}$ bis $R e_{D}=5,0 \times 10^{4}$. Die Strouhalzahlen sind aus Roshko (1961) und der Ablösewinkel aus Achenbach (1981, s.d. Abb. 14) entnommen.

Dass, in der Tat die Existenz freier abgelöster instabiler Scherschichten für die Beschreibung dieses Wechselwirkungsmechanismus eine notwendige Voraussetzung darstellt, ist leicht an dem instationären Ablöseverhalten der Zylinderströmung für Reynoldszahlen vor der Transition des Nachlaufs zu beobachten. Die Ausbildung der periodischen Ablösung vom Zylinder ist für diese Reynoldszahlen die Ursache einer globalen Instabilität der Grundströmung. Die Strouhalzahl bezogen auf die formale Rückkopplungslänge nimmt unterschiedliche Werte ein und ist von 1,4 deutlich verschieden (Tabelle 3.3 ). 


\begin{tabular}{|c|c|c|c|c|c|}
\hline $\operatorname{Re}_{D}$ & $S r$ & $\begin{array}{c}\varphi_{S, \text { oben }} \\
/[\mathrm{Grad}]\end{array}$ & $\begin{array}{l}\varphi_{S, \text { unten }} \\
/[\mathrm{Grad}]\end{array}$ & $\begin{array}{c}\Delta \varphi_{S} \\
/[\mathrm{Grad}]\end{array}$ & $S r_{f}$ \\
\hline $1,0 \times 10^{5}$ & 0,20 & 77 & 77 & 206 & 1,42 \\
\hline $2,0 \times 10^{5}$ & 0,21 & $(76+92) / 2$ & $(76+92) / 2$ & 192 & 1,41 \\
\hline $2,8 \times 10^{5}$ & 0,22 & $(80+96) / 2$ & $(80+96) / 2$ & 184 & 1,41 \\
\hline $3,2^{\times} \times 10^{5}$ & 0,18 & $(82+98) / 2$ & $(82+98) / 2$ & 180 & 1,13 \\
\hline $3,4 \times 10^{5}$ & 0,30 & 140 & $(78+94) / 2$ & 134 & 1,40 \\
\hline $4,0 \times 10^{5}$ & 0,50 & 140 & 140 & 80 & 1,40 \\
\hline $1,0 \times 10^{6}$ & 0,10 & ca. 130 & ca. 130 & 100 & 0,35 \\
\hline $3,0 \times 10^{6}$ & 0,21 & 85 & 85 & 190 & 1,39 \\
\hline
\end{tabular}

Tabelle 3.2 Berechnung der Strouhalzahl $S r_{f}$ von $R e_{D}=1,0^{\times} 10^{5}$ bis $R e_{D}=3,0^{\times} 10^{6}$. Die Strouhalzahlen sind aus Bild 3.1 und die Ablösewinkel sind aus den entsprechenden Anstrichbildern entnommen.

\begin{tabular}{|l|l|l|l|l|}
\hline \multicolumn{1}{|c|}{$R e_{D}$} & \multicolumn{1}{|c|}{$S r$} & \multicolumn{1}{|c|}{$\varphi_{S}$} & \multicolumn{1}{c|}{$\begin{array}{c}\Delta \varphi_{S} \\
/[\mathrm{Grad}]\end{array}$} & \multicolumn{1}{|c|}{$S r_{f}$} \\
\hline \hline 50 & 0,12 & 125 & 110 & 0,46 \\
\hline 60 & 0,135 & 122 & 116 & 0,54 \\
\hline 70 & 0,145 & 120 & 120 & 0,61 \\
\hline 80 & 0,152 & 119 & 122 & 0,64 \\
\hline 90 & 0,16 & 117 & 126 & 0,70 \\
\hline 100 & 0,165 & 116 & 128 & 0,74 \\
\hline 120 & 0,175 & 114 & 132 & 0,81 \\
\hline 140 & 0,18 & 112 & 136 & 0,85 \\
\hline
\end{tabular}

Tabelle 3.3 Berechnung der Strouhalzahl $S r_{f}$ vor der Transition des Nachlaufs für verschiedene Reynoldszahlen. Die Strouhalzahl wurde aus Fey (1997) [27] sowie Barkley und Henderson (1996) [6] entnommen. Der Ablösewinkel ist aus Achenbach 1979, [1] (s.d. Abb. 14) nach Rechnungen von Sucker und Bauer (1975) [87]. 
Die Strouhalzahl $S r_{f}$ wird mit der Anströmgeschwindigkeit gebildet. Dies geschieht natürlich, da diese Geschwindigkeit experimentell leicht zu bestimmen ist. Das gleiche gilt auch für die Wirbelablösefrequenz. Da die zeitliche Skalierung der Querwirbelentstehung aber mit ihrer Konvektivgeschwindigkeit verknüpft ist, sei noch die Strouhalzahl bezüglich Rückkopplungslänge und Konvektivgeschwindigkeit der Querwirbel $U_{c}$ dargestellt. Wird dabei beachtet, dass gilt $U_{c}=\lambda_{\mathrm{QW}} f$, dann folgt:

$$
S r_{L f / \lambda_{Q W}}=\frac{U_{\infty}}{U_{c}} \cdot S r_{f}=\frac{U_{\infty}}{U_{c}} \cdot \frac{L_{f}}{D} \cdot S r=\frac{U_{\infty}}{U_{c}} \cdot \frac{L_{f}}{D} \cdot \frac{U_{c}}{U_{\infty}} \cdot \frac{D}{\lambda_{Q W}}=\frac{L_{f}}{\lambda_{Q W}} .
$$

Wird davon ausgegangen, dass gilt $U_{c}=0,7 U_{\infty}$, so folgt für die Strouhalzahl $S r_{L f / \lambda_{Q W}}$ ein Wert von

$$
S r_{L f / \lambda_{Q W}}=2,0 \pm 0,1 \text {. }
$$

Mit diesen neu entwickelten Strouhalzahlen könnte es möglich sein, sowohl das instationäre und das zeitlich gemittelte Ablöseverhalten als auch die zu Grunde liegende Instabilitäten zu bestimmen. Diese Bestrebungen sind nicht neu. So führte z.B. Roshko (1954) [70] eine sog. universelle Strouhalzahl $S r_{R}=f b_{f} / \sqrt{1-C_{p B}} U_{\infty}$ ein. Sie wird gebildet aus der Ablösefrequenz $f$ und dem lateralen Abstand der freien abgelösten Scherschichten im Ablösegebiet $b_{f}$ sowie der zum Basisdruck nach der Bernoulli Gleichung entsprechenden Geschwindigkeit $U_{p B}$. Leider kann diese nicht den Widerstandsabfall bei konstanter Strouhalzahl im kritischen Bereich erklären. Darüberhinaus kann sie nicht den Wechselwirkungsmechanismus zwischen den Scherschichten und somit die Wirbelentstehung im Nachlauf des Zylinders beschreiben. Es ist denkbar, dass eine universelle Strouhalzahl in Abhängigkeit von der Reynoldszahl nur charakteristische Werte annehmen kann, die bestimmten Instabilitäten oder Wechselwirkungsmechanismen zugeordnet werden können. Dass eine derartige Zuordnung möglich scheint, kann auch aus einer neuen Darstellung der gewöhnlichen Strouhalzahl über dem Reziprok der Wurzel der Reynoldszahl abgelesen werden (Fey 1997 [27]). Hier sind es die Steigungen der Strouhalzahlverläufe, die eine Einteilung in unterschiedliche Instabilitätsbereiche bis $R e_{D}<2 \times 10^{4}$ erlauben Für den unterkritischen bis überkritischen Reynoldszahlbereich ergibt sich bei Querwirbelablösung ein konstanter Verlauf der Strouhalzahl, während bei Entstehung von Längswirbeln große positive Steigungen festzustellen wären. Im oberen Übergangsbereich würde sich allerdings eine große Streuung der Strouhalzahlen ergeben, so dass eine Angabe einer Steigung mit Unsicherheiten verknüpft wäre. Die Datenbasis für den transkritischen Bereich ist zu gering.

Um auf der Basis von Strouhalzahlen bei turbulentem als auch laminarem Nachlauf die Wechselwirkung der Scherschichten beruhend auf Instabilitäten verstehen zu können, bedarf es noch weiterer intensiver Untersuchungen.

\section{Großskalige Längswirbelstrukturen beim laminar-turbulenten Übergang}

Im kritischen und überkritischen Bereich ist zu beobachten, dass die spannweitig homogene Ablöseblase instabil werden kann. Dabei erweist sie sich als instabil gegenüber spannweitig periodischen sog. globalen Moden (nach Theofilis et al. 2000 [90]), die die Ausbildung spanweitig periodischer Längswirbelstrukturen begünstigen. Beim asymmetrisch abgelösten Strömungszustand im kritischen Bereich und im oberen Übergangsbereich ist dies zu erkennen. Kurz vor der „Transition $B^{“}$ (bei $R e_{D}=3,4 \times 10^{6}$, s. Bild 3.12 und Bild 3.13) wurde eine annähernd spannweitig periodische Wirbelstruktur mit einer spannweitigen Wellenlänge von $y_{L}=3 D$ auf der Zy- 
linderseite mit der transitionellen Ablöseblase festgestellt. Weitere spannweitig periodische abgelöste Wirbelstrukturen sind im oberen Übergangsbereich auszumachen. Sie bilden sich zellulär azimutal symmetrisch auf Ober- und Unterseite des Zylinders aus und besitzen unterschiedliche spannweitige Wellenlängen. So wurden im oberen Übergangsbereich mittlere Längen einer Zelle von $y_{L}=1,7 D$ bei $R e_{D}=0,4^{\times} 10^{6}$ (s. Bild 3.15) und von $y_{L}=1,2 D$ bei $R e_{D}=$ $0,79 \times 10^{6}$ (s. Bild 3.17) beobachtet. Insbesondere anhand der spannweitig periodischen Druckverteilungen für einen Azimutbereich von 95 Grad bis 115 Grad kan auf das räumlich periodische Ablösen von Längswirbeln geschlossen werden (s. Bild 3.21). Mit zunehmender Reynoldszahl nimmt die spannweitige Wellenlänge ab. Während bei einer Reynoldszahl von $R e_{D}=0,5^{\times} 10^{6}$ die spannweitige Länge etwa $y_{L}=2 D$ beträgt, hat sie bei $R e_{D}=0,8^{\times} 10^{6}$ nur noch eine Länge von $y_{L}=1 D$.

\section{Symmetrie der Zylinderablösung beim laminar-turbulenten Übergang}

Wenn immer drastische Änderungen im Verhalten der Zylinderströmung bevorstehen oder stattfinden, ist dies mit einer strukturellen Änderung der Ablösung verbunden. Diese Änderungen sind:

I. am Ende des unterkritischen Bereiches von der topologischen Ablösestruktur einer Ablöselinie zur doppelten Ablöseblase, d.h. zwei Ablöselinien mit einer dazwischenliegenden Anlegelinie,

II. am Ende des kritischen Bereiches von der spannweitig homogenen, azimutal symmetrischen Ablösung zu einer neuen spannweitig homogenen, azimutal symmetrischen Ablösung (nach „Transition A“ und „Transition B“), wobei sich auf jeder Seite des Zylinders eine transitionelle Ablöseblase ausbildet (überkritischer Zustand),

III. vom überkritischen zum transkritischen Bereich, wo mit Beginn des transkritischen Bereichs wieder eine spannweitig homogene und periodische, wenn auch turbulente Ablösung der Zylindergrenzschichtströmung erfolgt sowie

IV. zu Beginn des transkritischen Bereiches von der topologischen Ablösestruktur der doppelten Ablöseblase zu einer Ablöselinie auf jeder Seite des Zylinders.

In den Fällen I. bis III. erfolgt der Übergang von einem symmetrischen Zustand zu einem neuen symmetrischen Zustand aber nicht ohne einen oder mehrere „Zwischenzustände“. Vielmehr ist eine Brechung der Symmetrie festzustellen und der Charakter dieser „Zwischenzustände“ ist asymmetrisch:

I. Die Änderung der topologischen Ablösestruktur im unterkritischen Bereich vollzieht sich mit zunehmender Reynoldszahl zunächst erst auf einer Seite des Zylinders (s. Bild 3.5), um dann mit Erreichen der Reynoldszahl von $R e_{D}=2,0 \times 10^{5}$ auch auf der anderen Seite des Zylinders voll ausgebildet zu sein (s. Bild 3.4). Der „Zwischenzustand“ ist die Ausbildung einer azimutal asymmetrischen topologischen Ablösestruktur (s. Bild 3.5). Dieser Zwischenzustand scheint mit einer spannweitig periodischen Wirbelstruktur in der noch anliegenden Grenzschicht verknüpft zu sein. Die spannweitige Wellenlänge beträgt etwa $y_{L}=2 D$ (s. Bild 3.6). 
II. Bei Durchlaufen des kritischen Bereiches wird zunächst mit der „Transition A“ nur auf einer Seite eine Ablöseblase ausgebildet. Dieser Übergang ist verbunden mit einer Wechselwirkung der ablösenden Grenzschichten mit spannweitig periodischen kleinskaligen Längswirbelstrukturen der freien abgelösten instabilen Scherschichten. Die spannweitige Wellenlänge eines Längswirbelpaares beträgt kurz vor der „Transition $A^{\text {“ }}$ etwa $y_{L}=0,15 D$ (s. Bild 3.11). Erst mit weiter steigender Reynoldszahl entsteht (nach der „Transition $B^{“ \text { ) }}$ auch die Ablöseblase auf der anderen Seite des Zylinders. Im Unterschied zum unterkritischen Bereich liegt aber mit diesem azimutalen Symmetriebruch eine neue Qualität vor, da im zeitlichen Mittel nun nicht mehr der Auftriebsbeiwert verschwindet.

III. Der Übergang vom überkritischen zum transkritischen Bereich bedarf mehrerer „Zwischenzustände“", die sich in spannweitig periodischen abgelösten Längswirbelstrukturen unterschiedlicher spannweitiger Wellenlänge manifestieren. Dabei ist festzustellen, dass mit zunehmender Reynoldszahl die spannweitige Länge einer zellulären Wirbelstruktur kleiner wird. Es kann gesagt werden, dass der Symmetriebruch hier durch die Aufhebung der spannweitig homogenen Ablösung gekennzeichnet ist.

Damit stellt sich die Frage, ob es gelingt, durch geeignete stationäre oder instationäre, spannweitig homogene oder spannweitig periodische Störung der ablösenden Grenzschicht (auf einer Seite des Zylinders) eine bestimmte Instabilität anzuregen. Gelingt dies, müsste insbesonders die dadurch direkt oder indirekt verursachte Ausbildung von spannweitig periodischen Wirbelstrukturen in der ablösenden Grenzschicht zu einer Änderung des räumlich zeitlichen Ablöseverhaltens der Zylinderströmung führen.

Bei der Anwendung von spannweitig verteilten Wirbelerzeugern beim „Passiv“-Zylindermodell wird die stationäre Wirkung von Störungen untersucht. Mit Hilfe von Anstrichbildern und Kraftmessungen kann das räumlich zeitliche Verhalten ermittelt werden. Für die Umsetzung der aktiven Anregungverfahren wie das Absaugen von Grenzschichtfluid bzw. die akustische Anregung der Grenzschicht wird das ,Aktiv“-Zylindermodell genutzt. Das Erfassen des räumlich zeitlichen Ablöseverhaltens wird hier durch Messen der spannweitigen Wanddruckverteilung stromauf der Anregung erreicht. Welcher abgelöste Zustand sich einstellt, kann anhand der Druckschwankungen und des Basisdruckbeiwertes bestimmt werden. 


\section{Ergebnisse zum Verhalten der angeregten Zylindergrenzschicht}

Das räumlich zeitliche Verhalten der Zylinderströmung wird beim laminar-turbulenten Übergang geprägt durch das Instabilitätsverhalten der ablösenden Grenzschicht. Ähnlich wie Schubauer und Skramstadt (1947) [82], die die zweidimensionale Platten-Grenzschicht harmonisch störten, um die Existenz einer Grenzschicht-Instabilität gegenüber sog. Tollmien-Schlichting-Wellen (TS-Wellen) nachzuweisen, wird die Zylindergrenzschicht mit stationären bzw. harmonischen in Kombination mit spannweitig homogenen und spannweitig periodischen Störungen beaufschlagt. Wie viele andere Autoren in den vergangenen Jahrzehnten neben den TS-Wellen weitere sekundäre, dreidimensionale Instabilitätsformen durch gezieltes Anregen nachweisen konnten, soll auch mit den hier verwendeten Beeinflussungsbzw. Steuerungsverfahren die in der Zylindergrenzschicht inhärent existierenden Instabilitätsformen gefunden werden. Durch die angeregte Instabilität würde sich durch Entstehen von Wirbelstrukturen das Ablöseverhalten ändern. Mit Messung des Basisdruckes, der spannweitigen Wanddruckverteilung stromauf der künstlichen Störungseinbringung und mit Anstrichbildern sollen räumliche Skalen und instationäres Verhalten der Instabilitätsformen erfasst werden.

\subsection{Stationäre Störung mit spannweitig verteilten Wirbelerzeugern}

Bei der natürlich abgelösten Zylinderströmung sind im oberen Übergangsbereich spannweitig periodische zellartige Ablösestrukturen mit unterschiedlicher spannweitiger Länge zu beobachten - wie z.B. zu Beginn des oberen Übergangsbereiches mit $y_{L}=2 D$ (s. Bild 3.21). Mit der topologischen Darstellung dieser Ablösestruktur konnte geschlossen werden, dass je Zelle zumindest ein Längswirbelpaar vom Zylinder ablöst (s. Bild 3.23). Damit stellt sich die Frage, ob zum einen im unterkritischen Bereich eine spannweitig periodische Längswirbelstruktur mit dominanter Wellenlänge angeregt und zum anderen bis in den transkritischen Bereich erhalten werden könnte. In beiden Fällen wird eine Widerstandsreduktion erwartet. Im unterkritischen Bereich würden die spannweitig verteilten Wirbelerzeuger Längswirbel in der ablösenden Grenzschicht erzeugen. Damit könnte die ablösende Grenzschicht spannweitig inhomogen turbulent werden und ggf. wieder anlegen, um erst weiter stromab turbulent abzulösen. Aufgrund der weiter stromab erfolgenden, turbulenten Ablösung ist eine Widerstandsreduktion die Folge. Im überkritischen Bereich wird die transitionelle Ablöseblase auf jeder Seite des Zylinders durch die Störungen der Wirbelgeneratoren instabil, wodurch die turbulent ablösende Grenzschicht instabil gegenüber spannweitig periodischen Längswirbelstrukturen wird. Der damit verbundene Widerstandsbeiwert ist kleiner als im transkritischen Bereich. Regen die spannweitig verteilten Wirbelerzeuger nun gerade eine spannweitig periodische Längswirbelstruktur an, so besteht die Möglichkeit, dass diese bis in den transkritischen Bereich stabilisiert wird.

Mit dem „Passiv“-Zylindermodell werden Wirbelerzeuger mit einem spannweitigen Abstand von $\Delta y_{D}=2$ symmetrisch zur Zylindermitte angeordnet. Der spannweitige Abstand entspricht dabei der spannweitigen Länge einer Zelle der o.g. Ablösestruktur. Bei einer Azimutposition von $\varphi_{T G}=55$ Grad bzw. $\varphi_{T G}=80$ Grad erfolgt die Störung der anliegenden bzw. ablösenden Grenzschicht. Ist keine passive Störung gewollt, werden die Wirbelerzeuger ins Heck auf $\varphi_{T G}$ $=180$ Grad gedreht. 


\subsubsection{Widerstands- und Auftriebsbeiwertverhalten}

Das Widerstands- und Auftriebsbeiwertverhalten der passiv gestörten Zylinderströmung unterscheidet sich im Vergleich zu dem der ungestörten Zylinderströmung insbesondere im kritischen bis oberen Übergangsbereich (Bild 4.1). Im unterkritischen Bereich von $R e_{D}=1,0 \times 10^{5}$ bis $R e_{D}=2,0^{\times} 10^{5}$ ist eine geringe Widerstandserhöhung in beiden Fällen bei passiver Störung festzustellen. Die Widerstandserhöhung beträgt im Mittel etwa $\Delta C_{W}=0,1$. Der kritische Übergang vom azimutal symmetrischen zum azimutal asymmetrischen abgelösten Strömungszustand setzt bereits bei kleineren Reynoldszahlen ein. Der Übergang erfolgt hier nicht abrupt, sondern äußert sich in einem stetigen Ansteigen des Auftriebsbeiwertes ab einer Reynoldszahl von $\operatorname{Re}_{D}=2,0 \times 10^{5}$, die der kritischen Reynoldszahl der ,Transition A“ entsprechen würde. Somit kann der steigende Auftriebsbeiwert bei zunehmender Reynoldszahl mit der wachsenden Ausprägung einer transitionellen Ablöseblase auf der passiv gestörten Seite des Zylinders verbunden sein. Bei einer Reynoldszahl von etwa $R e_{D}=3,0 \times 10^{5}$ erreicht der Auftriebsbeiwert bei der passiv gestörten Zylinderströmung maximale Werte - für " $\varphi_{T G}=55 \mathrm{Grad}^{\prime \prime}$ einen Auftriebsbeiwert von etwa $C_{A}=0,55$ und für ", $\varphi_{T G}=80 \mathrm{Grad}^{\prime \prime}$ etwa $C_{A}=0,9$. Mit geringer Erhöhung der Reynoldszahl erfolgt eine drastische Änderung im asymmetrisch abgelösten Verhalten. In beiden Fällen kommt es zum Abfall im Widerstandsbeiwert auf etwa $C_{W}=0,4$ und im Auftriebsbeiwert auf etwa $C_{A}=-0,6$. Dies könnte der „Transition $B^{“}$ der ungestörten Zylinderströmung entsprechen. Andererseits muss der überkritische Zustand aufgrund der passiven einseitigen Störung instabil sein, so dass eine spannweitig homogen ausgebildete transitionelle Ablöseblase auf jeder Seite des Zylinders unwahrscheinlich ist. Das weitere Verhalten ist für beide Fälle ver-

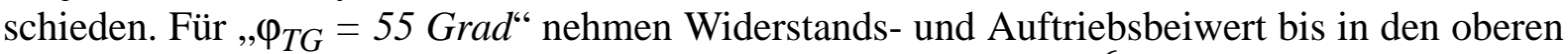
Übergangsbereich bis zu einer Reynoldszahl von etwa $R e_{D}=1,5^{\times} 10^{6}$ nur geringfügig zu, um mit weiter zunehmender Reynoldszahl deutlich anzusteigen. Für , $\varphi_{T G}=80 \mathrm{Grad}$ “ fällt zunächst nur der Auftriebsbeiwert auf etwa $C_{A}=-0,3 \mathrm{ab}$. Erst mit weiter zunehmender Reynoldszahl ab $R e_{D}=0,7 \times 10^{6}$ ist der weitere Anstieg im Widerstands- und Auftriebsbeiwert deutlich zu erkennen. Letztendlich ereicht der Widerstandsbeiwert für " $\varphi_{T G}=80 \mathrm{Grad}^{\prime \prime}$ im transkritischen Bereich einen Wert von etwa $C_{W}=0,65$ und für,$\varphi_{T G}=55 \mathrm{Grad}^{\prime \prime}$ einen Wert von etwa $C_{W}=0,5$. Der Auftriebsbeiwert verläuft im transkritischen Bereich nicht exakt bei Null, da insbesondere bei $\varphi_{T G}=55$ Grad die Wirbelerzeuger die anliegende Grenzschicht und somit die symmetrische Ablösung beeinträchtigen.

Die stationäre spannweitig periodische Störung durch die hier verwendeten passiven Wirbelerzeuger haben also in Abhängigkeit von ihrer Azimutposition relativ zur Ablösung eine unterschiedliche Wirkung auf das Ablöseverhalten der Zylinderströmung. Im unterkritischen Reynoldszahlbereich bewirken sie kein Turbulentwerden der Grenzschicht. Eher stellen die Wirbelerzeuger eine massive Störung der periodisch ablösenden Zylinderströmung dar. Die Erhöhung des Widerstandsbeiwertes könnte damit durch eine Verbreiterung des Nachlaufs erklärt werden. Ab einer Reynoldszahl von $R e_{D}=2,0^{\times} 10^{5}$ ist der symmetrische Strömungszustand der Ablösung zunehmend instabil gegenüber Störungen auf der Zylinderoberfläche. Die Ausbildung asymmetrisch abgelöster Strömungszustände ist die Folge. Auftriebserzeugende Ablösestrukturen müssten sich demnach auf der Oberseite des Zylinders ausbilden. Die Umkehr in dem Vorzeichen des Auftriebsbeiwertes bei der zweiten kritischen Reynoldszahl deutet auf das Entstehen einer Ablöseblase auf der Unterseite des Zylinders hin. Da diese instabil gegenüber spannweitig periodischen Wirbelstrukturen ist, ergibt sich eine neue Kräftebilanz. D.h., die 
Auftriebskräfte auf der Unterseite des Zylinders verursachen betragsmäßig einen größeren Auftrieb als die auf der Oberseite. Letztlich muss das Rezirkulationsgebiet schmaler geworden sein, da der Widerstandsbeiwert sprungartig kleiner geworden ist. Insbesondere bei ", $\varphi_{T G}=55 \mathrm{Grad}^{\text {" }}$ ist der Widerstands- und Auftriebsbeiwert im überkritischen bis oberen Übergangs-Reynoldszahlbereich nahezu konstant, so dass sich eine spannweitig periodische Ablösestruktur ausgebildet haben könnte. Bei weiterer zunehmender Reynoldszahl könnte die passive Störung nicht mehr ausreichen, um diesen spannweitig periodischen abgelösten Strömungszustand zu stabilisieren, so dass ab $R e_{D}=1,5^{\times} 10^{6}$ der Widerstandsbeiwert wie bei der ungestört abgelösten Zylinderströmung auf das Niveau im transkritischen Bereich wieder ansteigt.
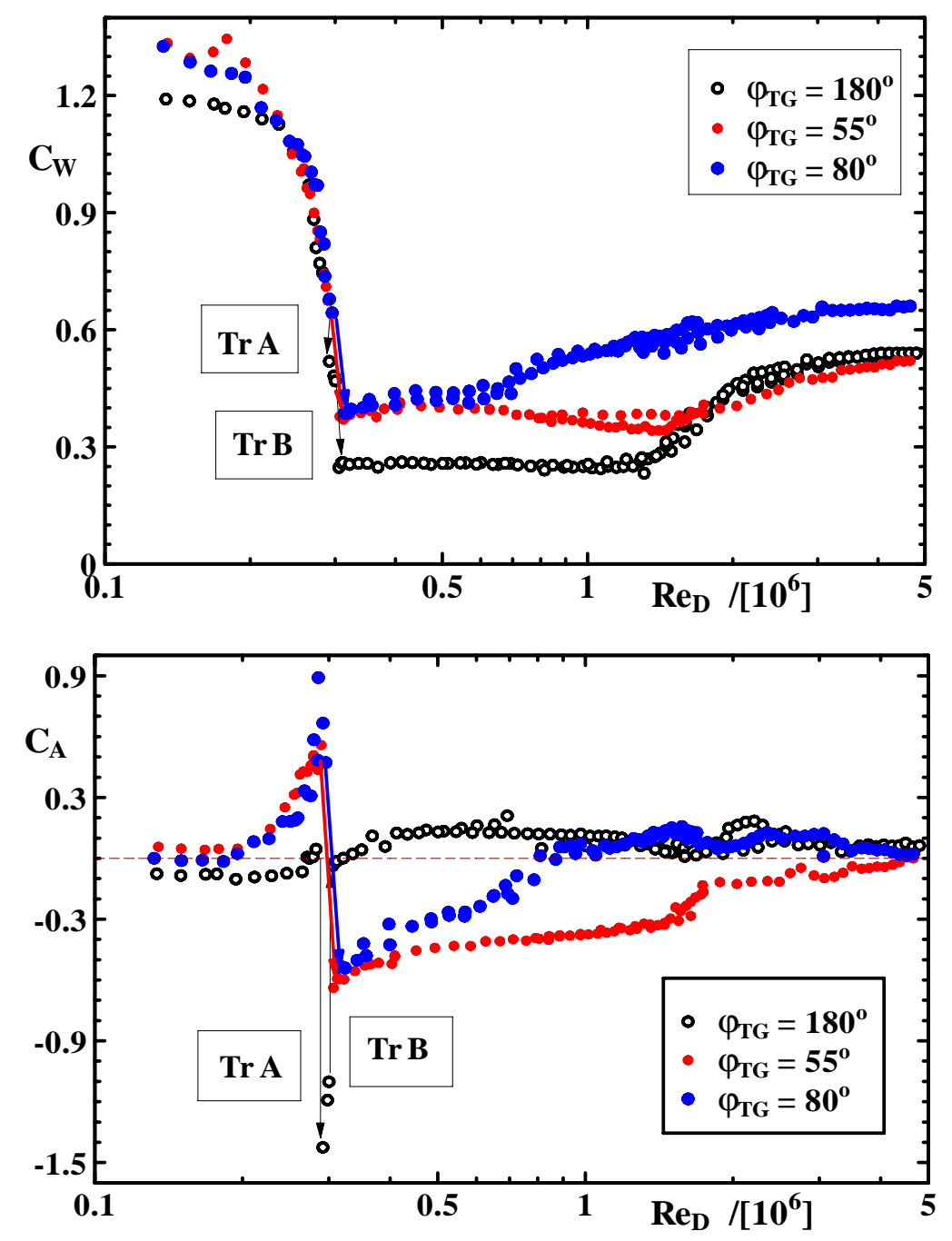

Bild 4.1 Widerstandsbeiwert (oben) und Auftriebsbeiwert (unten) in Abhängigkeit von der Reynoldszahl mit spannweitig verteilten Wirbelerzeuger im Abstand von $\Delta y_{T G}=$ $2 D$ bei zwei unterschiedlichen Azimutwinkeln bei $\varphi_{T G}=55$ Grad und $\varphi_{T G}=80$ Grad. Bei der Position $\varphi_{T G}=180$ Grad erfolgt keine passive Störung der abgelösten Zylinderströmung.

Das Anwachsen des Widerstandsbeiwertes für „, $\varphi_{T G}=80 \mathrm{Grad}^{\prime}$ könnte darauf zurückgeführt werden, dass mit zunehmender Reynoldszahl im überkritischen Reynoldszahlbereich die Grenzschicht im Vergleich zur natürlich abgelösten Zylinderströmung bereits bei kleineren Azi- 
mutwinkeln turbulent wird. Der transkritische Bereich wird also zu kleineren Reynoldszahlen verschoben. Die Wirbelerzeuger bewirken dann eine Störung der turbulent ablösenden Grenzschicht, so dass das Rezirkulationsgebiet und somit der Nachlauf breiter sind als im ungestörten Fall.

Wie bereits bei der natürlich abgelösten Zylinderströmung gesehen wurde, kann aus dem dynamischen Verhalten indirekt auf die Ablösestrukur geschlossen werden. Daher sind in Bild 4.2 die maximalen Schwankungsamplituden des Auftriebs für die jeweiligen Strouhalzahlen in Abhängigkeit von der Reynoldszahl für „, $\varphi_{T G}=80 \mathrm{Grad}$ “ dargestellt. Während im unterkritischen und anfänglich kritischen Bereich noch die periodische Wirbelablösung mit der Strouhalzahl von $\mathrm{Sr}=$ 0,2 dominiert, stehen für größere Reynoldszahlen als der zweiten kritischen Reynoldszahl die Wirbelablösung zu den Strouhalzahlen $S r=0,1$ und $S r=0,2$ in Konkurrenz zueinander. Da dieses konkurrierende dynamische Verhalten dem oberen Übergangsbereich der natürlich abgelösten Zylinderströmung zugeordnet werden kann, ist das Auftreten von spannweitig periodischen Ablösestrukturen wahrscheinlich. Darüberhinaus müssen die zellartigen Ablösestrukturen auf Ober- und Unterseite des Zylinders unterschiedliche spannweitige Längen aufweisen, damit ein von Null verschiedener zeitlich gemittelter Auftriebsbeiwert beobachtet werden kann. Eine andere Erklärung besteht in der prinzipiell asymmetrischen Ablösung der Zylinderströmung.

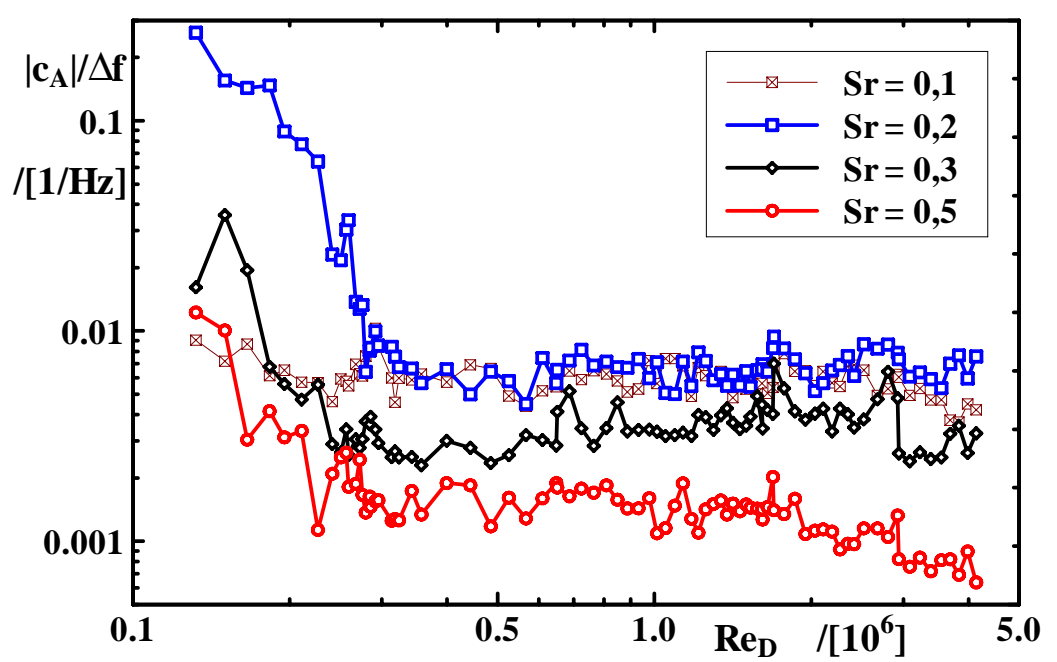

Bild 4.2 Schwankungsamplituden des Auftriebsbeiwertes für verschiedene Strouhalzahlen in Abhängigkeit von der Reynoldszahl bei passiver Störung durch spannweitig verteilte Wirbelerzeuger bei einem Azimutwinkel von $\varphi_{T G}=80 \mathrm{Grad}$

Aufgrund dieses Ablöseverhaltens muss eine Reihe von asymmetrischen Strömungszuständen existieren, die jeweils eine spannweitig unterschiedliche Ablösestruktur auf Ober- und Unterseite des Zylinders aufweisen müssten. Mit Hilfe der Anstrichtechnik sollen diese sichtbar gemacht werden.

\subsubsection{Asymmetrische Strömungszustände}

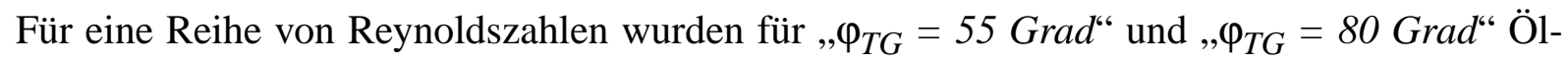
anstrichbilder angefertigt. Das Ziel besteht darin, zu zeigen, dass durch die einseitige Störung (auf der Oberseite) der Wirbelerzeuger sich eine entsprechend spannweitig periodische Wirbel- 
struktur ausgebildet hat. Wenn diese auf das globale Ablöseverhalten der Zylinderströmung eine stabilisierende Wirkung ausübt, so muss diese Ablösestruktur für , $\varphi_{T G}=80 \mathrm{Grad}$ “ für gröBere Reynoldszahlen als $R e_{k r i t, B}$ bis etwa $R e_{D}=0,7^{\times} 10^{6}$ und für " $\varphi_{T G}=55 \mathrm{Grad}^{\text {" }}$ bis zu einer Reynoldszahl von etwa $\operatorname{Re}_{D}=1,5^{\times} 10^{6}$ zu beobachten sein. Die Frage, die sich damit ergibt ist, welche spannweitg periodisch ablösende Wirbelstruktur wird dann auf der Unterseite festzustellen sein. Mit der Anwendung der Anstrichtechnik ist wieder zu beachten, dass aufgrund der zusätzlich aufgebrachten Oberflächenrauhigkeit durch die Suspension eine Verschiebung des Strömungszustandes zu kleineren Reynoldszahlen erfolgen kann. Ähnlich wie in 3.4 Übergang vom überkritischen zum transkritischen Bereich wird auch hier dem Anstrichbild ein entsprechender Strömungzustand über die Bestimmung der Kraftbeiwerte während der Entstehung des Anstrichbildes zugeordnet.

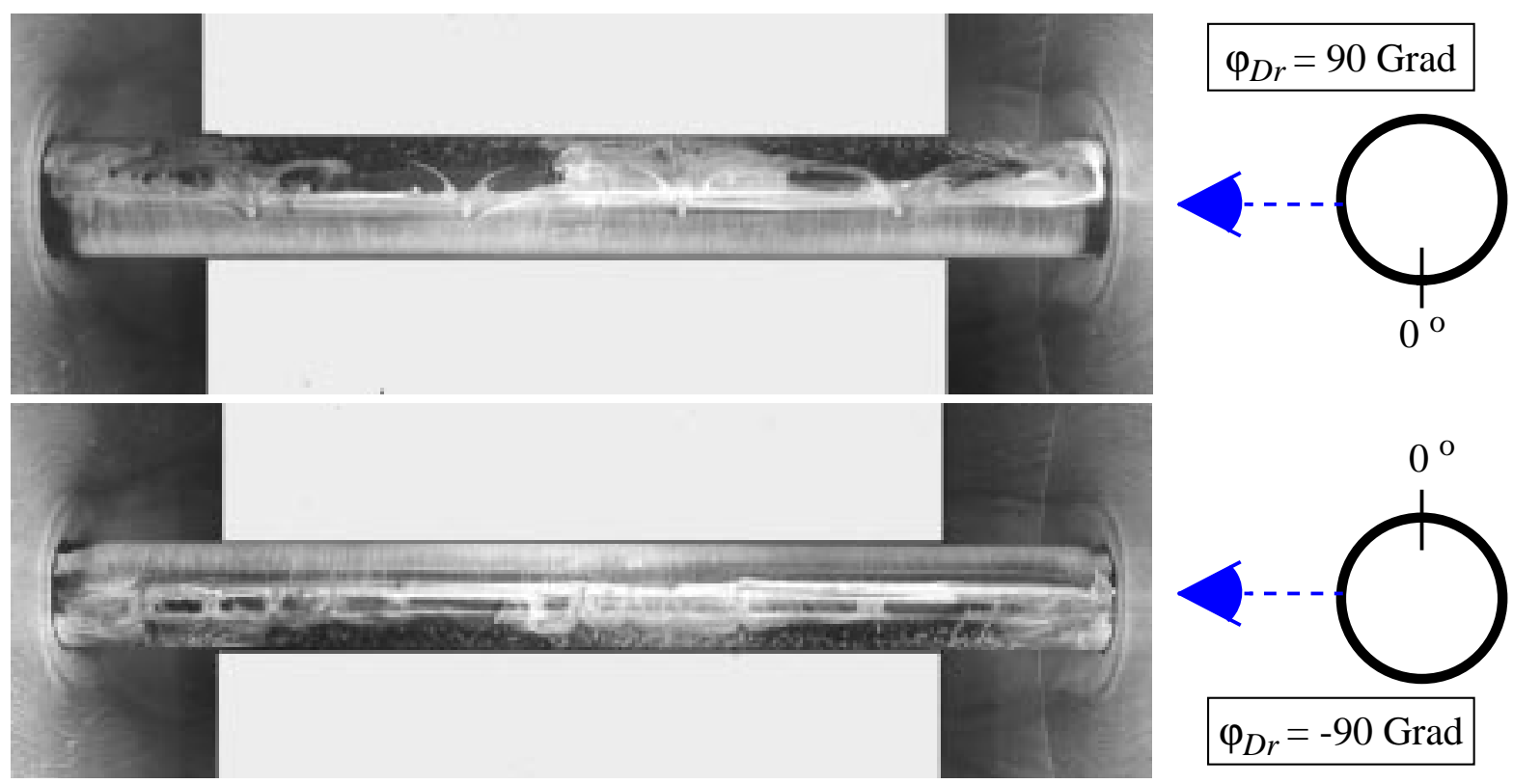

Bild 4.3 Anstrichbild bei $R e_{D}=0,32^{\times} 10^{6}$ mit einer Draufsicht unter einem Azimutwinkel von +90 Grad (oben) und -90 Grad (unten). Die azimutale Position der Wirbelerzeuger ist $\varphi_{T G}=80$ Grad. $C_{W}=0,45$ (s.a. Bild A4.1). Auf der passiv gestörten Seite bildet sich eine spannweitig periodische Ablösestruktur mit $y_{L}=2 \mathrm{D}$ und auf der Unterseite eine spannweitig zelluläre Ablösestruktur aus.

Beginnend mit dem Anstrichbild bei einer gemessenen Reynoldszahl von $R e_{D}=0,32^{\times} 10^{6}(\underline{\text { Bild }}$ $\underline{\text { 4.3 }}$ ), über $\operatorname{Re}_{D}=0,40^{\times} 10^{6}$ (Bild 4.4) bis zu einer Reynoldszahl von $\operatorname{Re}_{D}=0,52^{\times} \times 10^{6}(\underline{\text { Bild 4.5 }})$ wird beobachtet, dass auf der Oberseite des Zylinders durch die spannweitig periodisch verteilten Wirbelerzeuger eine bekannte Ablösestruktur mit einer spannweitigen Wellenlänge von etwa $y_{L}=2 D$ sich ausbildet. Auf der Unterseite hingegen scheint sich die Ablösestruktur zu etablieren, die der natürlich abgelösten Zylinderströmung in diesem Strömungszustand entsprechen würde. Die Ablösestruktur auf der Unterseite in Bild 4.3 bei einer gemessenen Reynoldszahl von etwa $R e_{D}=0,32^{\times} 10^{6}$ erinnert an die im kritischen Bereich mit azimutal asymmetrischer natürlich abgelöster Strömung. Mit weiter zunehmender Reynoldszahl entsprechen die gemachten Anstrichbilder bereits Strömungszuständen des oberen Übergangsbereiches im Vergleich zu der natürlich abgelösten Zylinderströmung. So kann in Bild 4.4 bei der gemessenen Reynoldszahl von $R e_{D, A n s t r}=0,40^{\times} 10^{6}$ auch auf der Unterseite eine spannweitig periodische 
Ablösestruktur mit $y_{L}=2 D$ festgestellt werden. Darüberhinaus scheinen aber noch weitere spannweitige Strukturen dieser Ablösestruktur überlagert zu sein.

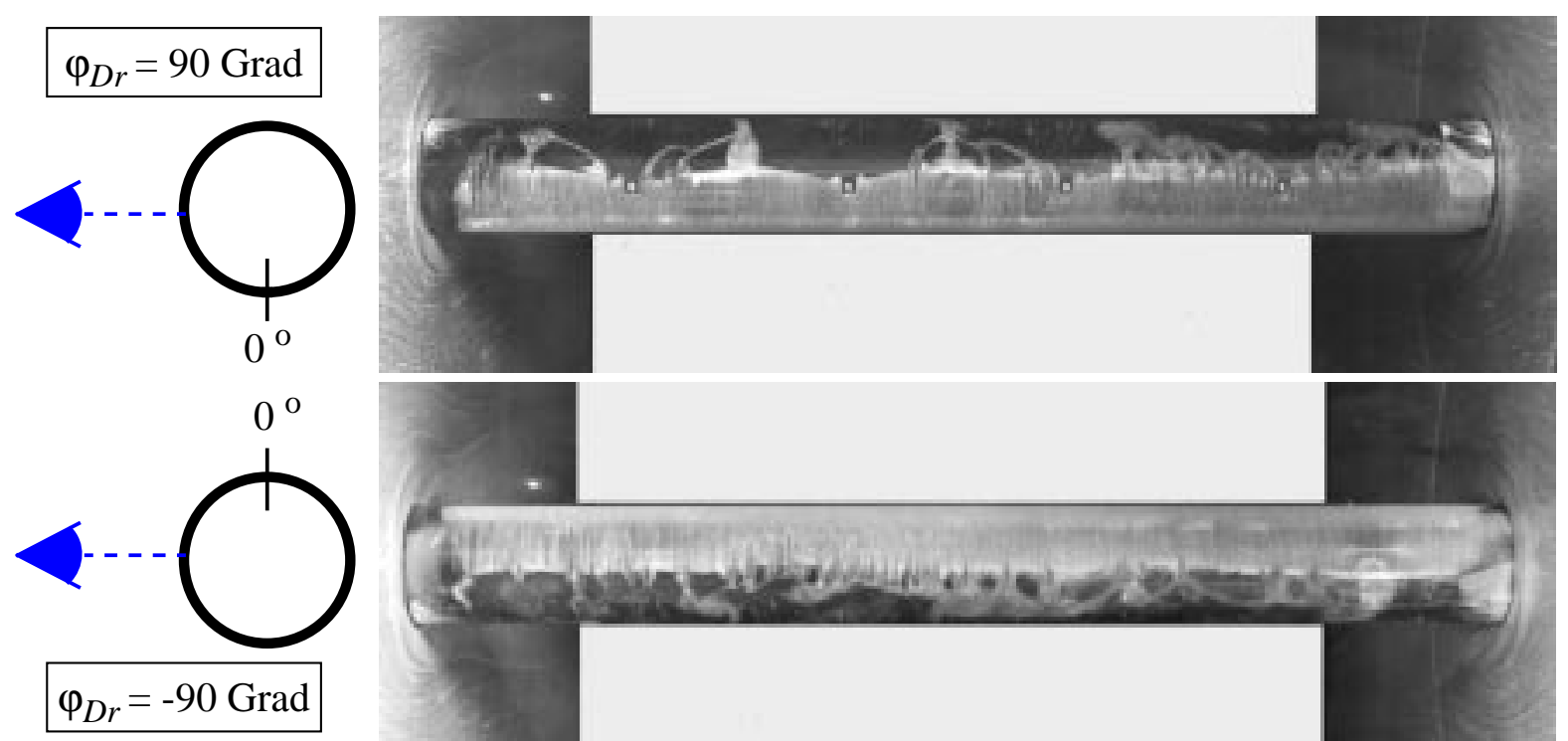

Bild 4.4 Anstrichbild bei $R e_{D}=0,40^{\times} 10^{6}$ mit einer Draufsicht unter einem Azimutwinkel von +90 Grad (oben) und -90 Grad (unten). Die azimutale Position der Wirbelerzeuger ist $\varphi_{T G}=80$ Grad. $C_{W}=0,52$ (s.a. Bild A4.2). Auf der Unterseite ist eine Überlagerung vom Wirbelstrukturen unterschiedlicher spannweitiger Wellenlänge zu erkennen.
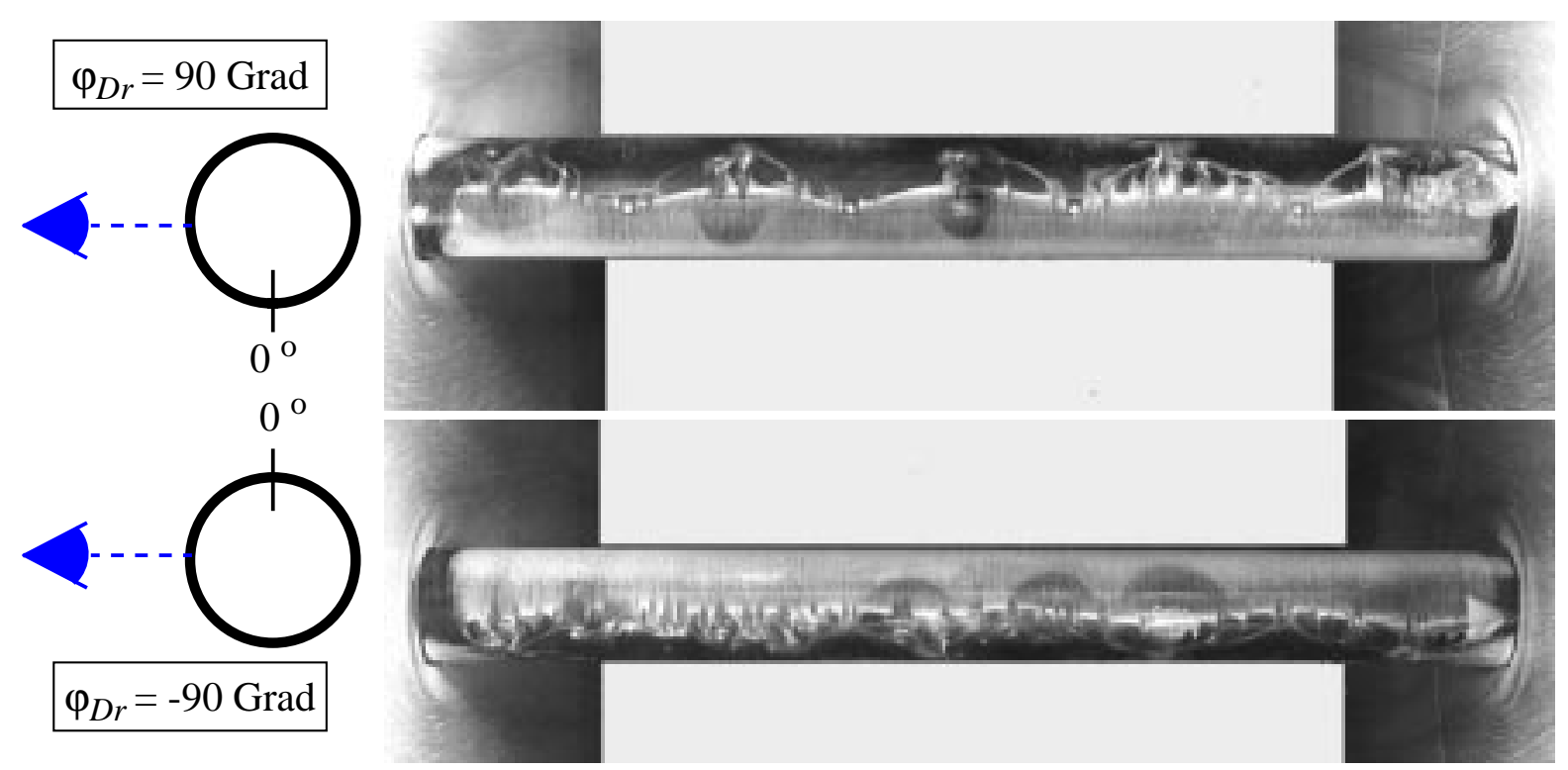

Bild 4.5 Anstrichbild bei $R e_{D}=0,52^{\times} 10^{6}$ mit einer Draufsicht unter einem Azimutwinkel von +90 Grad (oben) und -90 Grad (unten). Die azimutale Position der Wirbelerzeuger ist $\varphi_{T G}=80$ Grad. $C_{W}=0,55$ (s.a. Bild A4.3). Auf der Unterseite ist eine zellartige Ablösestruktur mit einer spannweitigen Wellenlänge von $y_{L}=1,2 \mathrm{D}$ zu beobachten. 
Bei weiterer Erhöhung der Reynoldszahl scheint sich diese Ablöseverhalten fortzusetzen, wobei die spannweitige Länge einer zellulären Ablösestruktur auf der Unterseite doch deutlich abnimmt (siehe Bild 4.5) . Bei $\operatorname{Re}_{D}=0,69 \times 10^{6}$ (Bild 4.6) kann nur noch vage von einer zellulären Ablösestruktur sowohl auf der Unter- als auch auf der passiv gestörten Oberseite gesprochen werden. Vielmehr müsste hiermit der Übergang in den transkritischen Bereich erfolgen. Mit passiver Störung kommt es aber scheinbar nicht zu der Ausbildung spannweitig homogen ablösender Querwirbel. Die periodischen Auftriebsschwankungen bei $S r=0,2$ haben den gleichen Betrag wie die bei $S r=0,1$ (siehe Bild 4.2), so dass eher Querwirbel und Längswirbel das Ablöseverhalten konkurrierend bestimmen.

Obwohl also die spannweitige Ablösestruktur auf der Unterseite des Zylinders mit der Reynoldszahl sich ändert, ist nach der „Transition $B$ “ für einen großen Reynoldszahlbereich ein nahezu konstanter Verlauf im Widerstands- und Auftriebsbeiwert zu verzeichnen. Das würde bedeuten, dass die durch die Wirbelerzeuger angeregte spannweitig periodische Längswirbelstruktur global das Ablöseverhalten dominiert und diesen asymmetrischen Strömungzustand aufrechterhalten kann. Erst mit Erreichen einer bestimmten Reynoldszahl, für , $\varphi_{T G}=80 \mathrm{Grad}^{\text {“ }}$ bei einer Reynoldszahl von etwa $R e_{D}=0,7 \times 10^{6}$, wird die spannweitig periodische Ablösestruktur auf der Oberseite instabil. Die Begründung liegt darin, dass nunmehr die laminar-turbulente Transition der (ablösenden) Grenzschicht stromauf der azimutalen Position der Wirbelerzeuger stattfindet. Die noch anliegende Zylindergrenzschicht ist turbulent und kann stromauf der Ablösung offenbar nicht mehr spannweitig periodisch angeregt werden.

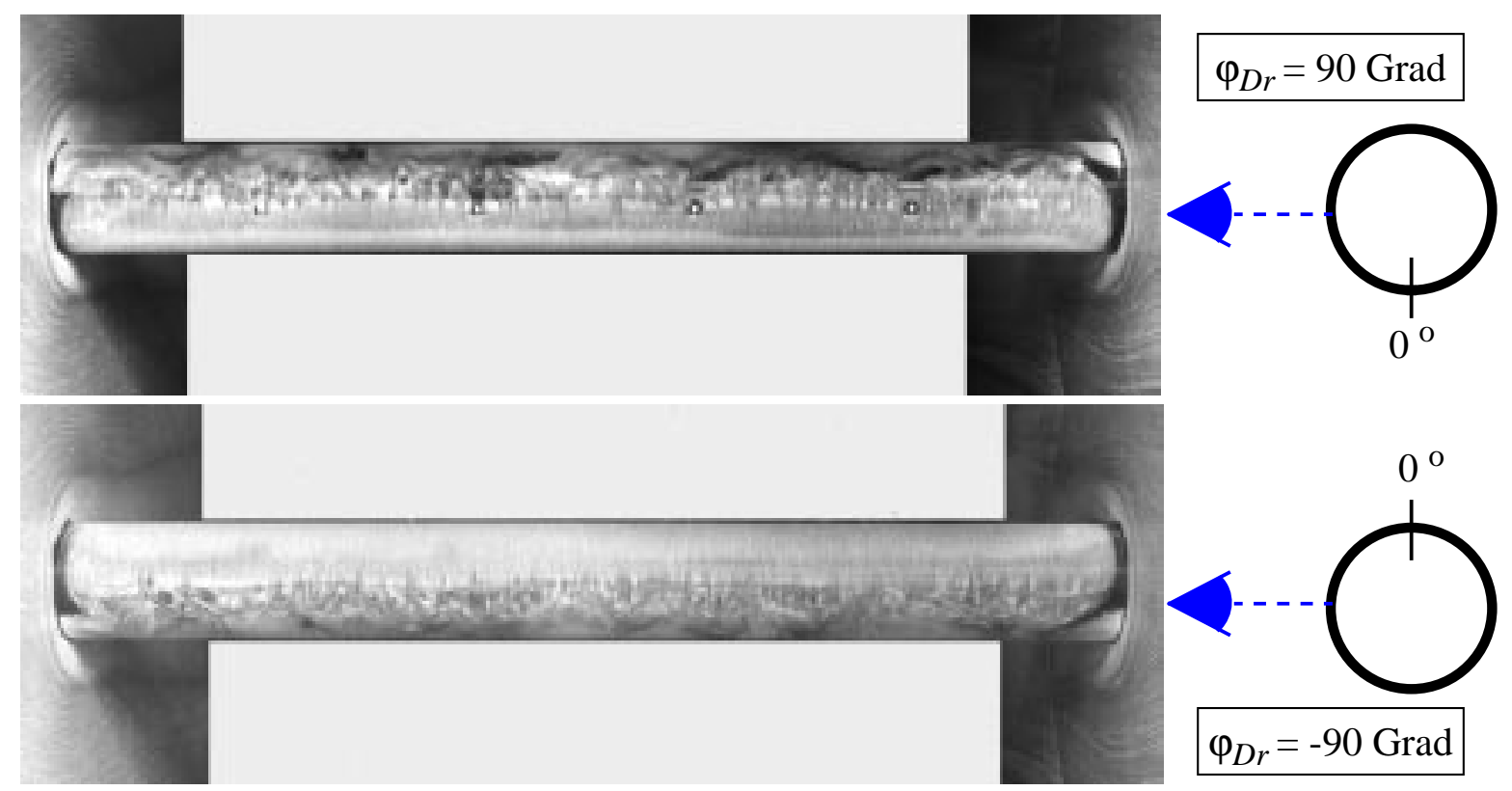

Bild 4.6 Anstricbild bei $R e_{D}=0,69 \times 10^{6}$ mit einer Draufsicht auf den Zylindern unter einem Azimutwinkel von $+90 \mathrm{Grad}$ (oben) und $-90 \mathrm{Grad}$ (unten). Die azimutale Position der Wirbelerzeuger ist $\varphi_{T G}=80$ Grad. $C_{W}=0,63$ (s.a. Bild A4.4). Sowohl auf der Unter- als auch auf der Oberseite ist eine spannweitig periodische Ablösestruktur kaum noch zu erkennen.

In Analogie zu , $\varphi_{T G}=80 \mathrm{Grad}^{\prime \prime}$ ergibt sich auch für,$\varphi_{T G}=55 \mathrm{Grad}^{\prime \prime}$ ein ähnliches Verhalten. Zur Betrachtung der Ölanstrichbilder sei an dieser Stelle auf den Anhang A.4 Passiv gestörte Zylindergrenzschicht durch Wirbelerzeuger verwiesen. So ist auch für " $\varphi_{T G}=55$ Grad“" zu be- 
obachten, dass sich auf der Oberseite des Zylinders die bekannte Ablösestruktur mit einer spannweitigen Wellenlänge von $y_{L}=2 D$ ausbildet, während auf der Unterseite sich eher eine Ablösestruktur entwickelt, die zu der Reynoldszahl der natürlich abgelösten Zylinderströmung gehört (s. Bild A3.2 bis Bild A3.4). Lediglich scheint die spannweitig periodische Ablösestruktur auf der Oberseite des Zylinders für einen größeren Reynoldszahlbereich stabil zu sein. Selbst bei einer gemessenen Reynoldszahl von $R e_{D}=0,94 \times 10^{6}$ (s. Bild A3.4) ist auf der Oberseite des Zylinders deutlich diese Ablösestruktur mit $y_{L}=2 D$ zu erkennen. Auch hier kann die Erklärung, wie für,$\varphi_{T G}=80 \mathrm{Grad}^{\prime \prime}$ Anwendung finden. Im transkritischen Bereich wandert der Ort der Transition in der anliegenden Grenzschicht mit zunehmender Reynoldszahl stromauf. Aus den Anstrichbildern ist zu entnehmen, dass zwischen den Reynoldszahlen $R e_{D}=0,94 \times 10^{6}$ (s. Bild A3.4) und $R e_{D}=1,60^{\times} \times 10^{6}$ (s. Bild A3.5) die Transition in der Grenzschicht die azimutale Position der Wirbelerzeuger passieren muss.

\subsubsection{Spannweitige Steuerung spannweitig periodischer Ablösestrukturen}

Abschließend sei noch auf ein Phänomen hingewiesen, was die Wirkungsweise der spannweitig periodisch verteilten Wirbelerzeuger verdeutlicht. Der Abstand benachbarter Wirbelerzeuger beträgt $\Delta y_{T G}=2 D$. Da die Spannweite des Zylinders $L=10 D$ ausmacht, ergeben sich unter Berücksichtigung der Symmetrie zwei spannweitige Anordnungen von gleichverteilten Wirbelerzeugern. Bei der ersten Variante müssten fünf Wirbelerzeuger bei den spannweitigen Positionen $y_{D}=0, \pm 2$ und \pm 4 verwendet werden, während bei der zweiten Variante vier Wirbelerzeuger bei den spannweitigen Positionen $y_{D}= \pm 1$ und \pm 3 eingesetzt werden könnten. Mit der Vorstellung, dass ein Wirbelerzeuger die Quelle für die Ausbildung von einem Paar Längswirbel ist, die dann für die Ausbildung einer Zelle der Ablösestruktur verantwortlich sind (siehe auch Bild 3.23), wurde die zweite Variante benutzt. Diese vermutete Wirkung der Wirbelerzeuger zeigt sich in den Anstrichbildern aber nicht. Wie beispielsweise in Bild 4.4 zu sehen ist, bewirkt ein Wirbelerzeuger eine spannweitig lokale Störung der spannweitig homogenen Ausbildung der Ablöseblase, was bisher auch als „Durchbruch“ bezeichnet wurde. Hier allerdings kommt es zu einem vorzeitigen Ablösen der noch laminaren Grenzschicht, während in den spannweitig benachbarten Gebieten die Ablöseblase erhalten bleibt und die Grenzschicht erst weiter stromab turbulent ablöst. Durch die spannweitig periodische Anordnung der Wirbelerzeuger entsteht somit diese spannweitig periodische Ablösestruktur. Die damit verbundene Ablösung von paarweisen Längswirbeln erfolgt somit zwischen zwei Wirbelerzeugern. So ergibt sich zwar ein nahezu einheitliches Bild der Ausbildung einer spannweitig periodischen Ablösestruktur bei einer Reynoldszahl von $R e_{D}=0,40^{\times} 10^{6}$ für alle drei Fälle, aber die spannweitige Lage der Zellen ist mit,$\varphi_{T G}=55 \mathrm{Grad}^{\prime \prime}$ und,$\varphi_{T G}=80 \mathrm{Grad}^{\prime}$ "gegenüber,$\varphi_{T G}=180 \mathrm{Grad}^{\prime}$ um eine halbe Wel-

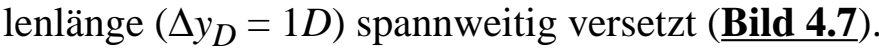

Zusammenfassend kann gesagt werden, dass mit spannweitig periodisch verteilten stationären Störungen durch Wirbelerzeuger sonst eine natürlich auftretende spannweitig periodische abgelöste Längswirbelstruktur im überkritischen bis oberen Übergangs-Reynoldszahlbereich angeregt werden kann. Obwohl sie nur auf einer Seite des Zylinders die Grenzschichtentwicklung beeinflusst, bewirkt sie eine Stabilisierung des globalen Ablöseverhaltens der Zylinderströmung. Die stabilisierende Wirkung reicht dabei von überkritischen Reynoldszahlen bis zu derart großen Reynoldszahlen, bei denen die Grenzschicht am Ort der Wirbelerzeuger noch nicht tur- 
bulent ist. Bei einer azimutalen Position der Wirbelerzeuger von $\varphi_{T G}=80 \mathrm{Grad}$ wurde diese Wirkung bis zu einer Reynoldszahl von etwa $R e_{D}=0,6^{\times} 10^{6}$ beobachtet, während sie bei $\varphi_{T G}=$ 55 Grad bis etwa $R e_{D}=1,5^{\times} 10^{6}$ erhalten bleibt.

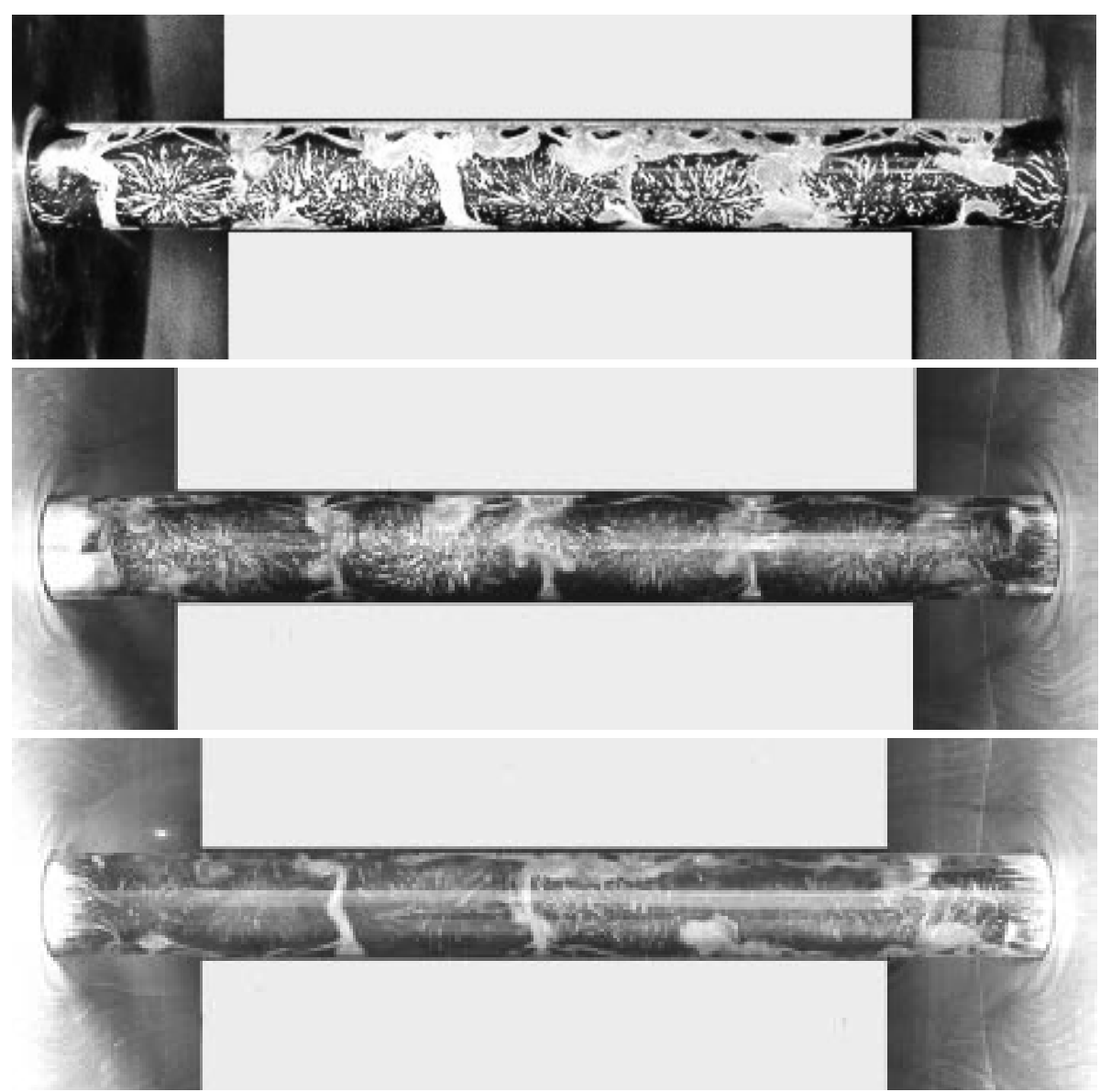

Bild 4.7 Anstrichbild bei $R e_{D}=0,40^{\times} 10^{6}$ mit einer Draufsicht unter einem Winkel von etwa $\varphi_{D r}=180 \mathrm{Grad}$. Azimutwinkel der Wirbelerzeuger $\varphi_{T G}=180 \mathrm{Grad}$ mit $C_{W}=0,34$ (oben), $\varphi_{T G}=55 \mathrm{Grad}$ mit $C_{W}=0,45$ (Mitte), $\varphi_{T G}=80 \mathrm{Grad}$ mit $C_{W}=0,52$ (unten). Aufgrund der Wirkungsweise der Wirbelerzeuger (s. Text) bildet sich die spannweitig periodische Wirbelstruktur bei passiver Störung im Vergleich zu der natuürlcih abgelösten Zylinderströmung um eine halbe Wellenlänge spannweitig versetzt aus.

Die stabilisierende Wirkung wurde dabei zunächst aufgrund des nahezu konstanten Widerstands- und Auftriebsbeiwertes über diese Reynoldszahlbereiche vermutet (Bild 4.1). Mittels Anstrichbilder konnte die zugehörige Ablösestruktur aufgezeigt und die Wirkung bestätigt werden. Gerade mit ", $\varphi_{T G}=55$ Grad" wird deutlich, dass mit einer spannweitig periodischen passiven Störung stromauf der Ablösung bis in den transkritischen Bereich die Ausbildung einer spannweitig periodischen Ablösestruktur erhalten werden kann. Der damit verbundene Widerstandsbeiwert ist im Vergleich zur ungestörten Zylinderströmung geringer, so dass dieses passive Steuerungsverfahren zur Widerstandsreduktion eingesetzt werden könnte. 


\subsection{Stationäre Störung der Grenzschicht durch Absaugen}

Eine weitere Möglichkeit der stationären Störung der Grenzschicht besteht durch Absaugen. Dazu wird das ,Aktiv“-Zylindermodell benutzt (siehe 2.2.3 „Aktiv“-Zylindermodell). Das Absaugen erfolgt dabei über Kammern des Aktuatormoduls, die durch kleine Bohrungen in der Zylinderwand mit der Oberfläche verbunden sind. Mit den 36 separat betreibbaren Kammern sind verschiedene spannweitige Konfigurationen des Absaugens realisierbar. Darüberhinaus kann das Absaugevolumen und damit die Störamplitude variiert werden. In Analogie zur passiven Beeinflussung durch spannweitig periodisch verteilte Wirbelerzeuger wird auch hier der Frage nachgegangen, ob durch eine spannweitig periodische stationäre Störung eine räumlich großskalige, spannweitig periodische Ablösestruktur angeregt werden kann. Im Vergleich dazu wird auch spannweitig homogen abgesaugt, um die unterschiedliche Wirkung auf die ablösende Grenzschicht beurteilen zu können. Die Störung der laminaren Grenzschicht erfolgt auf einer Seite des Zylinders, in der Nähe der Ablösung bei einem Azimutwinkel von $\varphi_{E}=75 \mathrm{Grad}$. Das im Vergleich zur natürlich abgelösten Zylinderströmung veränderte Verhalten des Basisdruckbeiwertes liefert Aussagen über die Effektivität der aktiven Beeinflussungsmaßnahme. Die stromauf der Anregung bei einem Azimutwinkel von $\varphi_{P}=55 \mathrm{Grad}$ gemessene spannweitige Wanddruckverteilung gestattet das spannweitige zeitlich gemittelte wie instationäre Ablöseverhalten zu beschreiben. Das Absaugen wird generell zu Beginn der Messreihe bei $\operatorname{Re}_{D}=1,0 \times 10^{5}$ eingeschaltet und mit Ende der Messreihe bei etwa $R e_{D}=4,0^{\times} 10^{5}$ wieder abgeschaltet.

\subsubsection{Spannweitig homogene stationäre Störung durch Absaugen}

Mit der spannweitig homogenen Anwendung wird über alle Kammern des Aktuatormoduls abgesaugt. Der Volumenstrom beträgt $Q_{E}=1,92 \mathrm{~m}^{3} / \mathrm{h}$. Um die Effektivität der Anregungsverfahren auch bei Anwendung unterschiedlicher Parameterwerte vergleichen zu können, wird die mittlere Widerstandsreduktion $\overline{\Delta C_{W, r e l}}$ im Vergleich der beeinflussten $C_{p B, E}$ zu der ungestörten Zylinderströmung aus den entsprechenden Basisdruckbeiwertverläufen unter Ausnutzung von $\{3.1\}$ berechnet. Sie ergibt sich zu

$$
\overline{\Delta C_{W, r e l}}=-\frac{1}{R e_{D, 2}-R e_{D, 1}} \int_{\operatorname{Re}_{D, 1}}^{R e_{D, 2}} \frac{C_{p B, E}-C_{p B, 0}}{C_{p B, 0}} d R e_{D} .
$$

Dabei wird über einen Reynoldszahlbereich von $R e_{D, 1}=1,0^{\times} 10^{5}$ bis $R e_{D, 2}=3,4 \times 10^{5}$ gemittelt. Dies entspricht einem großen Reynoldszahlausschnitt aus dem unterktitischen Bereich bis zu der kritischen Reynoldszahl der „Transition $B_{“}$. Im Vergleich der gestörten zu der natürlich abgelösten Zylinderströmung wird eine wesentliche Verbesserung im Basisdruckbeiwert für einen Reynoldszahlbereich von $R e_{D}=2,0^{\times} 10^{5}$ bis $R e_{D}=3,5^{\times} 10^{5}$ festgestellt (Bild 4.8d). Der Basisdruckbeiwert nimmt bei diesen Reynoldszahlen einen nahezu konstanten Wert von etwa $C p_{B}=$ $-0,6$ an. Die mittlere Widerstandsreduktion beträgt $\overline{\Delta C_{W, r e l}} \approx 18 \%$. Der Grund für dieses Verhalten des Basisdruckbeiwertes ist in der azimutalen Druckverteilung bei $y_{D}=0$ (Bild 4.8a) und auch in den spannweitigen Druckverteilungen bei $\varphi_{P}=55$ Grad (Bild 4.8b) ablesbar. Die azimutale Druckverteilung zeigt bereits ab der Reynoldszahl von $R e_{D}=1,0^{\times} 10^{5}$, aber insbesondere ab $R e_{D}=2,0^{\times} 10^{5}$ eine deutliche Ausprägung des Druckminimums bei etwa $\varphi=75$ Grad. Diese azimutal asymmetrische Druckverteilung entspricht der Verteilung des asymmetrischen Zustandes im kritischen Reynoldszahlbereich. Ebenso ist ab $R e_{D}=2,0 \times 10^{5}$ eine nahezu spannweitig 
homogene Verteilung des Druckbeiwertes zu beobachten. Lediglich an den seitlichen Begrenzungen des Zylinders treten Störungen auf, die das zeitlich gemittelte als auch das instationäre Ablöseverhalten beeinträchtigen.
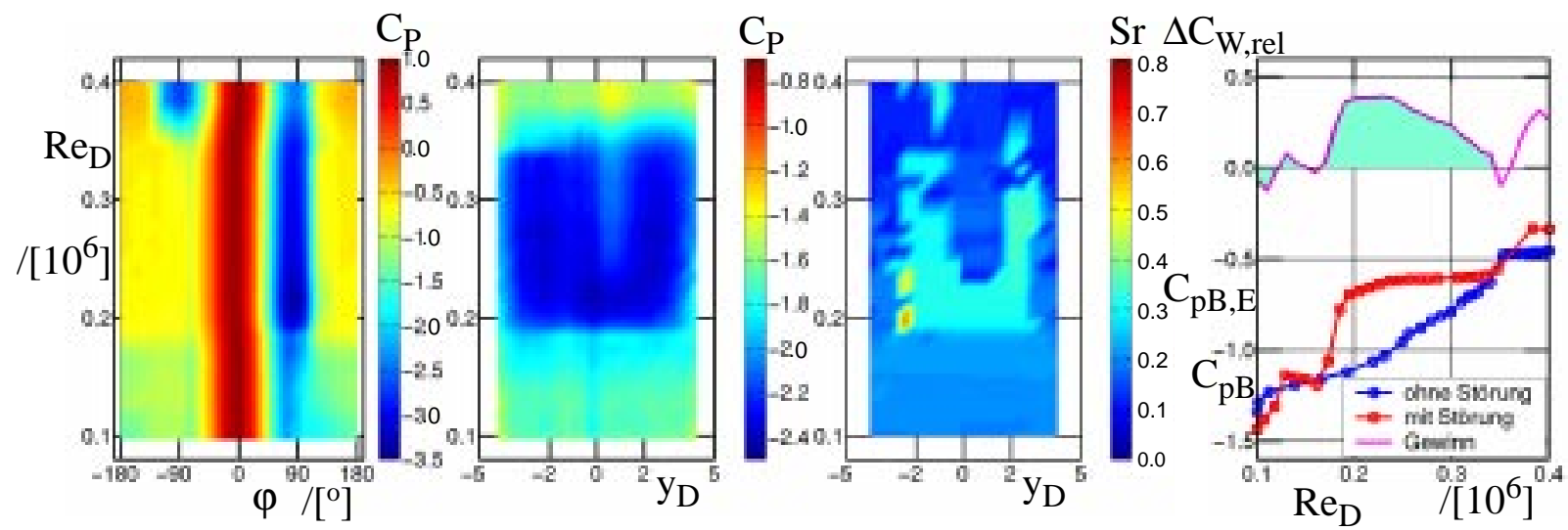

Bild 4.8 Kontinuierliches spannweitig homogenes Absaugen mit einem Volumenstrom von $Q_{E}=1,92 \mathrm{~m}^{3} / \mathrm{h}$. Von links nach rechts: (a) Azimutale, (b) spannweitige Wanddruckverteilung, (c) spannweitige Verteilung der Strouhalzahl der dominanten Wirbelablösefrequenz sowie (d) Basisdruckbeiwertverläufe der natürlich abgelösten und gestörten Zylinderströmung mit Verlauf der relativen Widerstandsreduktion. Die mittlere Widerstandsreduktion beträgt $\overline{\Delta C_{W, \text { rel }}} \approx 18 \%$.

Während im symmetrischen Zustand die periodische Ablösung periodisch mit $S r=0,20$ spannweitig homogen erfolgt, dominiert im asymmetrischen Zustand die periodische Ablösung mit $S r=0,31$, wenn auch nur in einem inneren spannweitigen Bereich (Bild 4.8c). Mit weiter zunehmender Reynoldszahl bleibt zwar der asymmetrische Zustand erhalten, die Druckschwankungen zu $S r=0,31$ nehmen aber ab und niederfrequente Fluktuationen bestimmen zunehmend das instationäre Verhalten. In Bild 4.9 sind die drei Zustände mit ihrem typischen spannweitigen zeitlich gemittelten wie dynamischen Verhalten herausgegriffen. Daraus ist ablesbar, dass die Druckschwankungen vom unterkritischen zum überkritischen Zustand drastisch abnehmen.
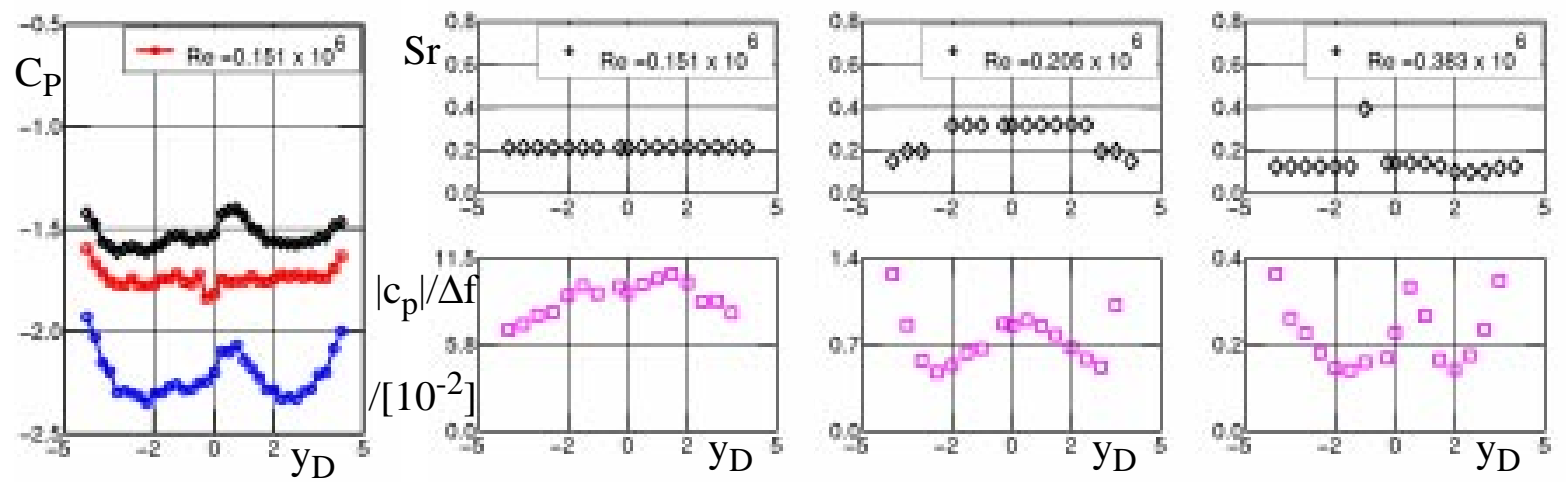

Bild 4.9 Spannweitiges zeitlich gemitteltes (links) und (rechts davon) instationäres Verhalten anhand der Strouhalzahl und der Druckschwankungen für die drei möglichen Symmetriezustände der Ablösung - symmetrisch (unterkritisch) bei $R e_{D}=1,5^{\times} 10^{5}$, asymmetrisch (kritisch) bei $R e_{D}=3,2^{\times} 10^{5}$ und symmetrisch (überkritisch) bei $R e_{D}=$ $3,8^{\times} 10^{5}$. 
Der Übergang vom unterkritischen zum kritischen Zustand erfolgt aufgrund der Absaugung bereits bei kleineren Reynoldszahlen. Das bedeutet, dass die ablösende Grenzschicht aufgrund der spannweitig homogene Störung instabil wird. Mit Erreichen einer dafür entsprechenden kritischen Reynoldszahl löst die Grenzschicht zunächst laminar ab, wird turbulent und legt turbulent wieder an. Erst mit noch größer werdender Verzögerung der Grenzschicht erfolgt schließlich turbulenten Ablösen. Durch die eingebrachte Störung wird also ein asymmetrisch abgelöster Zustand erzwungen, der der natürlich abgelösten asymmetrischen Zylinderströmung entspricht. Weiterhin erfolgt trotz der zusätzlichen Störung der Grenzschicht keine Erhöhung der Druckschwankungen.

\subsubsection{Spannweitig periodische stationäre Störung durch Absaugen}

Mit dem Aktuatormodul des „Aktiv“-Zylinders wurden verschiedene spannweitig periodische Absaugeverteilungen eingestellt. Insbesondere wurden zwei spannweitige Abstände mit $y_{E}=$ 2,2D und $y_{E}=4,4 D$ jeweils mit maximaler (,max") und keiner Absaugung (,min") in der Zylindermitte $\left(y_{D}=0\right)$ realisiert (Bild 4.10) . Das Ergebnis der Störung der Grenzschicht wird wieder anhand der spannweitigen und azimutalen Druckverteilungen sowie der Basisdruckverteilungen diskutiert. Um die Effektivität der unterschiedlichen Konfigurationen miteinander vergleichen zu können, wurde der Volumenfluss der Absaugung mit $Q_{E}=1,92 \mathrm{~m}^{3} /$ $\mathrm{h}$ als auch die Anzahl der aktivierten Kammern, durch die abgesaugt wird, mit $N=12$ konstant gehalten.

Ähnlich wie bei der spannweitig homogenen Absaugung ist auch hier die Ausbildung einer asymmetrischen Ablösung nahezu spannweitig homogen ab $R e_{D}=2,0^{\times} 10^{5}$ ausgeprägt, wenn von Randeinflüssen abgesehen wird. Im Vergleich zur spannweitig homogenen Absaugung erreicht der Basisdruckbeiwert nach dem Übergang bis zum überkritischen Bereich auch hier den entsprechenden Basisdruckbeiwert von etwa $C_{p B}=-0,6$. Für kleinere Reynoldszahlen bezüglich des asymmetrischen Überganges sind in den spannweitigen Druckverteilungen periodische Modulationen zu erkennen. Im spannweitigen Verlauf existieren spannweitige Abschnitte mit miminimalen oder maximalen Druckbeiwert. Die Bereiche mit minimalem Druckbeiwert entsprechen dem Druckbeiwert des asymmetrisch abgelösten Zustandes, während die mit maximalem Druckbeiwert dem symmetrisch abgelösten Zustand zuzuordnen sind. In diesen Reynoldszahlbereich wird ebenfalls schon eine Verbesserung des Basisdruckbeiwertes erreicht, so dass die mittlere Widerstandsreduktion etwas größer ausfällt und etwa $\overline{\Delta C_{W, \text { rel }}} \approx 25 \%$ beträgt. Die Änderungen im Druckbeiwert treten dabei im Mittel bei den Spannweiten auf, wo auch abgesaugt wird. Werden die spannweitigen Abschnitte mit minimalen Druckbeiwert ausgemessen, so ergeben sich bestimmte typische spannweitige Längen: 3,3D, 2,5D, 1,7D (s.a. Anhang A.5 Re-gemittelte Widerstandsreduktionen bei aktiver Beeinflussung). Diese spannweitig periodischen Druckverteilungen erlauben zwei Interpretationen. Die eine ist, dass spannweitig mehr oder weniger periodisch symmetrisch und asymmetrische abgelöste Zustände aneinander abwechseln. Eine andere Interpretation ist, dass spannweitig periodisch sich in der anliegenden Grenzschicht ausbilden und zu diesem Muster der spannweitigen Verteilung des Wanddruckes führen. Da die dominanten Druckschwankungen bei einer Strouhalzahl von $S r=0,2$ beobachtet werden, muss vor dem asymmetrischen Übergang eine symmetrische Ablösung erfolgen (Bild 4.11). 

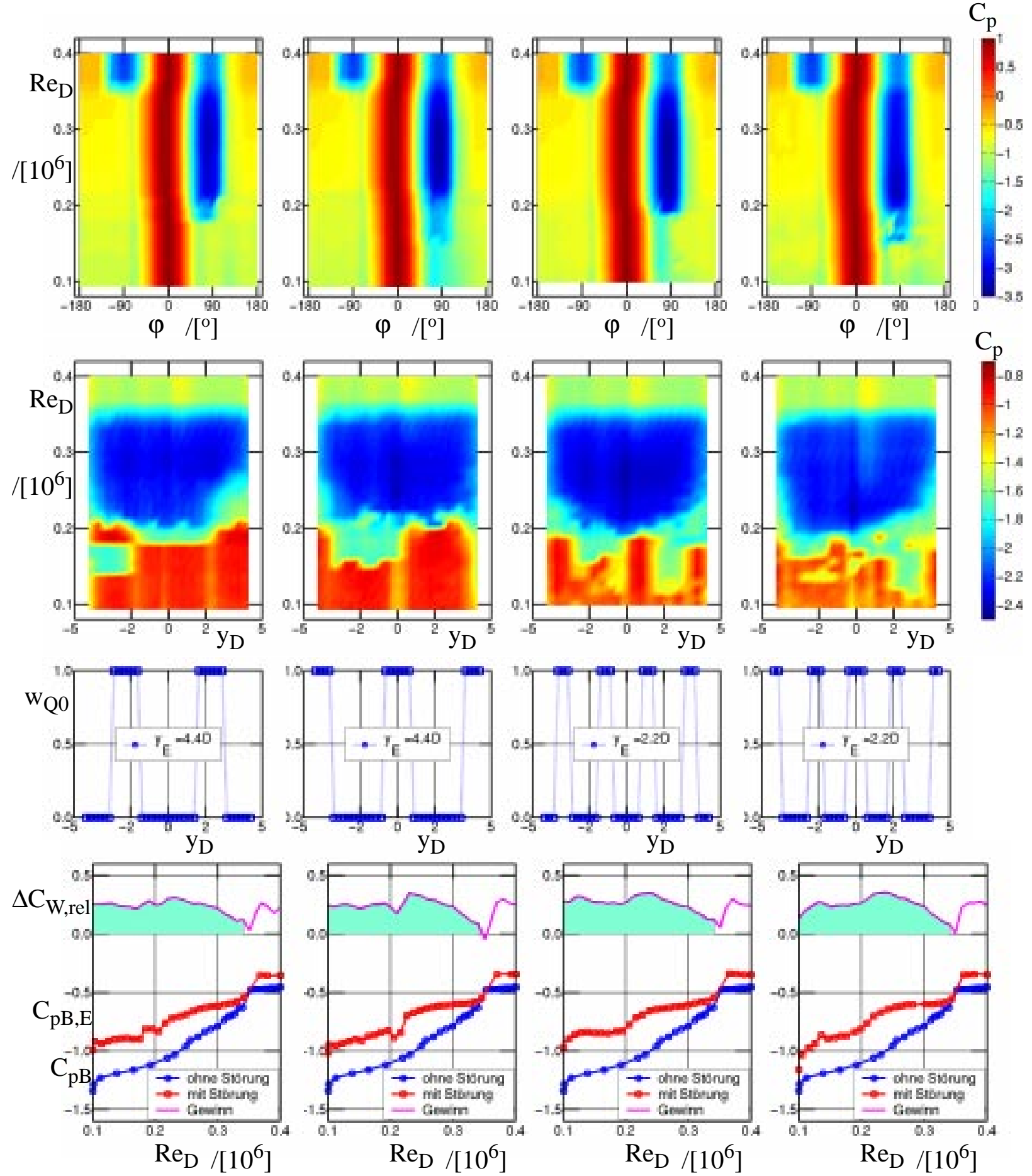

Bild 4.10 Von oben nach unten: Darstellung der azimutalen, spannweitigen Druck- und der Verteilung der Absaugegeschwindigkeit bei $Q_{E}=1,92 \mathrm{~m}^{3} / \mathrm{h}$ sowie Basisdruckbeiwertverläufe bei Absaugung im Vergleich zur ungestörten abgelösten Strömung bei Anwendung verschiedener spannweitiger Konfigurationen - von links nach rechts: $y_{E}=4,4 D(\min )$ mit $\overline{\Delta C_{W, r e l}} \approx 24 \%, y_{E}=4,4 D(\max )$ mit $\overline{\Delta C_{W, r e l}} \approx 24 \%, y_{E}=$ $2,2 D$ (min) mit $\overline{\Delta C_{W, \text { rel }}} \approx 26 \%, y_{E}=2,2 D($ max $)$ mit $\overline{\Delta C_{W, \text { rel }}} \approx 25 \%$. 
Darüber hinaus sind mit den spannweitigen Bereichen minimalen Druckbeiwertes auch geringere Druckschwankungen verbunden wie es im Bild 4.11 für $R e_{D}=1,85^{\times} 10^{5}$ geziegt wird. Sie sind aber immer noch bei $S r=0,2$ die dominanten Druckschwankungen. Mit der Ausbildung des asymmetrisch abgelösten Zustandes hingegen sind eindeutig die größten Druckschwankungen bei $S r=0,31$ festzustellen. Der asymmetrische Zustand bildet sich dabei nahezu über der gesamten Spannweite aus, wobei zu den seitlichen Begrenzungen hin niederfrequente Druckschwankungen die asymmetrische Ablösung beeinträchtigen (Bild 4.11).
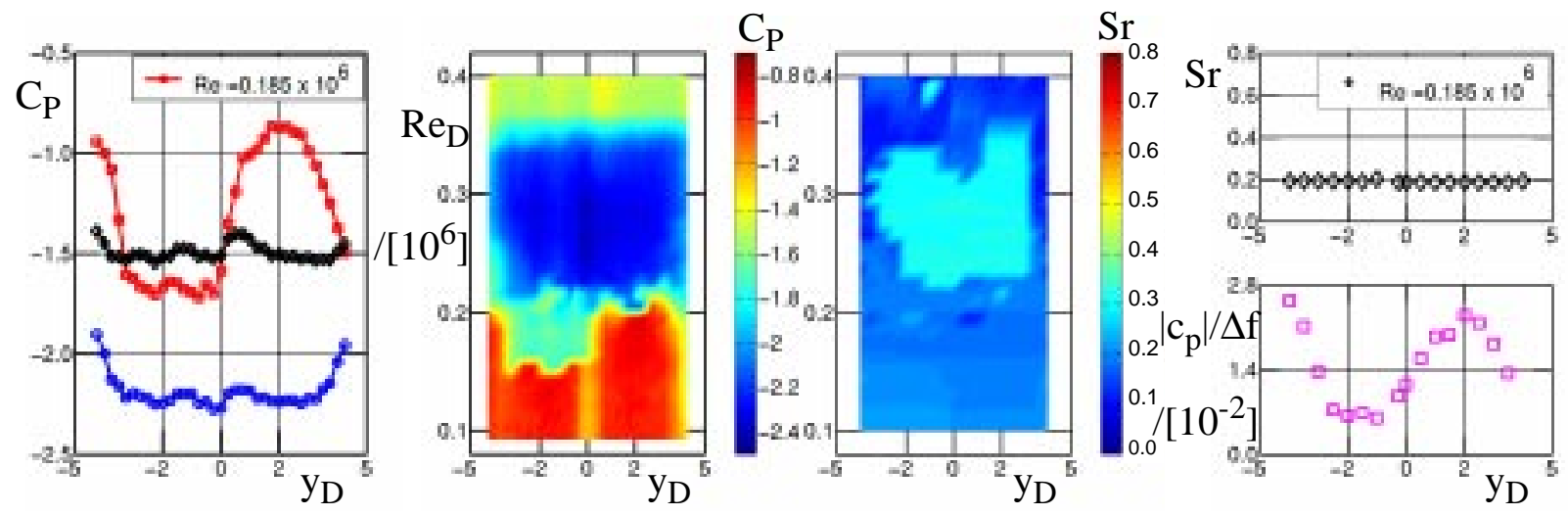

Bild 4.11 Von links nach rechts: Darstellung von charakteristischen spannweitigen Druckverteilungen bei $R e_{D}=1,85^{\times} 10^{5}$ für symmetrische (rote Kurve), bei $R e_{D}=3,2^{\times} 10^{5}$ asymmetrische (blaue Kurve) und bei $R e_{D}=3,8^{\times 10^{5}}$ (schwarze Kurve) wieder symmetrische Ablösung bei Absaugung mit $y_{E}=4,4 D$ (max) (s.a. Bild 4.10); Übersichtsdarstellung der spannweitigen Druckverteilung und der Verteilung der Strouhalzahl der dominantenten Druckschwankungen in Abhängigkeit von der Reynoldszahl und (ganz rechts) für $R e_{D}=1,85^{\times} 10^{5}$ die charakteristische Verteilung der Strouhalzahl und der zugehörigen Amplitude der Druckschwankungen.

\subsubsection{Einfluss der Variation der Absaugegeschwindigkeit}

Wie bei der bisherigen Anwendung des kontinuierlichen Absaugens im spannweitig homogenen als auch bei den spannweitig periodischen Konfigurationen gesehen wurde, wird in dem Reynoldszahlbereich von $R e_{D}=1,0^{\times} 10^{5}$ bis $R e_{D}=2,0^{\times} 10^{5}$ der asymmetrisch abgelöste Zustand nicht erreicht. Es liegt nahe, dass die Ursache in einer zu geringen lokalen Absaugegeschwindigkeit besteht. Weiterhin stellt sich die Frage, ob mit zunehmender Absaugerate der Übergang vom symmetrisch zum asymmetrisch abgelösten Zustand zu kleineren Reynoldszahlen hin verschoben werden kann. Daraufhin wurde die Absaugerate für alle vier Fälle variiert.

Allgemein kann festgestellt werden, dass tatsächlich mit Erhöhung der Absaugegeschwindigkeit insbesondere in dem Reynoldszahlbereich von $\operatorname{Re}_{D}=1,0^{\times} 10^{5}$ bis $\operatorname{Re}_{D}=2,0^{\times} 10^{5}$ eine weitere Verbesserung im Basisdruckbeiwert erreicht werden kann. Damit verbunden sind zwei Effekte, die im folgenden anhand einer Konfiguration mit $y_{E}=2,2 D(\max )$ stellvertretend diskutiert werden. Die verwendeten Volumenflüsse betragen $Q_{E}=1,37 \mathrm{~m}^{3} / \mathrm{h}$ und $Q_{E}=4,40 \mathrm{~m}^{3} / \mathrm{h}$.

In Bild 4.12 sind die spannweitige Druckverteilung und der Basisdruckbeiwert in Abhängigkeit von der Reynoldszahl für die beiden Volumenflüsse dargestellt. Für die kleinere Absaugerate ergibt sich eine mittlere Widerstandsreduktion von etwa $\overline{\Delta C_{W, \text { rel }}} \approx 22 \%$, während für die ver- 
gleichsweise fast dreifache Absaugerate eine mittlere Widerstandsreduktion von etwa $\overline{\Delta C_{W, r e l}} \approx 26 \%$ zu verzeichnen ist. Die erhöhte Reduktion im Widerstandsbeiwert ist maßgeblich auf eine weitere Verbesserung des Basisdruckbeiwertes in dem Reynoldszahlbereich von $R e_{D}=1,0^{\times} 10^{5}$ bis $R e_{D}=2,0^{\times} 10^{5}$ zurückzuführen. Für diesen Reynoldszahlausschnitt verhält sich der Basisdruckbeiwert ebenfalls nahezu konstant und nimmt einen Wert von etwa $C_{p B}=-$ 0,9 an. Die Ursache für dieses Verhalten ist aus der stromauf der Absaugung gemessenen spannweitigen statischen Druckverteilung ablesbar.

Bei Absaugung mit dem größeren Absaugevolumen ist bereits ab Reynoldszahlen von etwa $R e_{D}$ $=1,4 \times 10^{5}$ eine nahezu spannweitig periodische Druckverteilung bis zum kritischen Übergang zu beobachten. Bei den spannweitigen Positionen von etwa $y_{D}=-3,5$ bis $y_{D}=-1,5$, von $y_{D}=-$ 0,5 bis $y_{D}=0,5$ und von $y_{D}=+1,8$ bis $y_{D}=+3,3$ bildet sich jeweils ein lokales Druckminimum aus. Der zweite Effekt ist deutlich in einer weiteren Ausdehnung des asymmetrischen nahezu spannweitig homogenen Zustandes zu erkennen. Während bei der geringeren Absaugerate der Übergang bei einer Reynoldszahl von etwa $R e_{D}=2,1 \times 10^{5}$ einsetzt, erfolgt dieser bei der größeren Absaugerate bereits bei etwa $\operatorname{Re}_{D}=1,8^{\times} 10^{5}$.
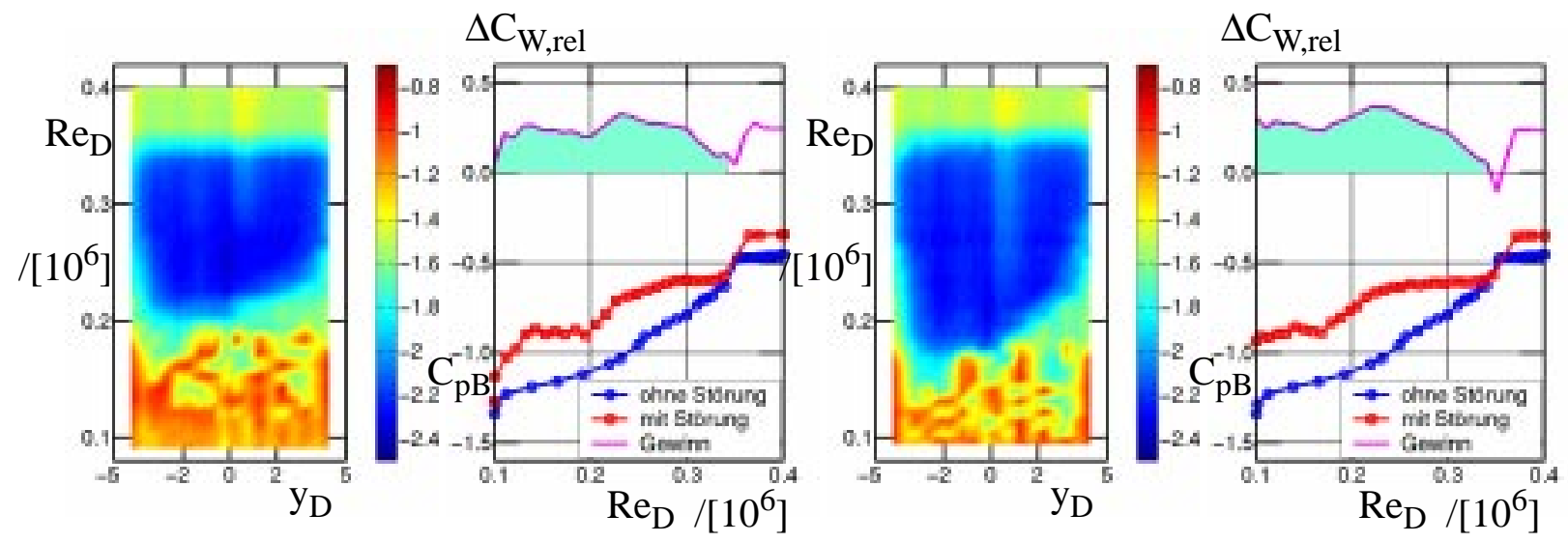

Bild 4.12 Spannweitige Druckverteilungen und Basisdruckbeiwertverläufe in Abhängigkeit von dem Absaugevolumenstrom bei einer spannweitig periodischen Absaugung von $y_{E}=2,2 D:$ mit $Q_{E}=1,37 \mathrm{~m}^{3} / \mathrm{h}$ wird eine mittlere Widerstandsreduktion von $\overline{\Delta C_{W, \text { rel }}} \approx 22 \%$ erreicht (links) und mit $Q_{E}=4,40 \mathrm{~m}^{3} / \mathrm{h}$ eine von $\overline{\Delta C_{W, \mathrm{rel}}} \approx 26 \%$ (rechts).

Daraus kann geschlossen werden, dass sich die asymmetrische Ablösung sich über den Zwischenzustand einer spannweitig periodischen Ablösung bei um so kleineren Reynoldszahlen im unterkritischen Bereich etablieren kann, je größer die Störamplitude ist. Welcher Mechanismus bewirkt aber zum einen das spannweitig periodische Verhalten vor dem Übergang und zum anderen den Übergang zum spannweitig homogenen asymmetrischen Zustand ?

Dazu sei nochmal die Art und Weise der Absaugung genauer betrachtet. Die Absaugung erfolgt durch kleine Bohrungen je Kammer. Der Durchmesser eines Loches gegegeben zu $d_{P}=0,3 \mathrm{~mm}$ liegt dabei in der Größenordnung der Grenzschichtdicke am Ort der Ablösung. Diese Geometrie stellt eine Quelle für die Ausbildung eines Längswirbelpaares dar (Messing et al. 1999 [48]), die sich in der noch anliegenden und gerade sich ablösenden laminaren Grenzschichströmung ausbilden. Darüberhinaus könnten sich nebeneinander befindende Längswirbelpaare zu größeren in Strömungsrichtung orientierten Wirbeln vereinigen, wie Böttcher und Wedemeyer (1989) [14] dieses Phänomen in einem Wasserkanal hinter Sieben beobachteten. Erfolgt nun die Anre- 
gung mit ausreichender Amplitude spannweitig periodisch kann sich eine spannweitig periodische Längswirbelstruktur ausbilden. Dabei scheinen nur bestimmte spannweitige Wellenlängen möglich zu sein. D.h. die Längswirbelstrukturen wechselwirken mit der ablösenden Grenzschicht nur spannweitig lokal, wobei global symmetrische Ablösung statffindet. Erst mit weiter zunehmender Reynoldszahl wird der symmetrische Zustand instabil und der asymmetrische Zustand bestimmt das globale Ablöseverhalten.

\subsection{Instationäre Störung der Grenzschicht durch akustische Anregung}

Die instationäre Störung wird durch akustische Aktuatoren realisiert. Sie bewirken eine zeitlich periodische Druckschwankung an der Wand des Zylinders. Mit einer Reihe von separat betreibbaren Kammern längs der Spannweite ist somit eine kombinierte spannweitig und zeitlich periodische Anregung der Grenzschicht möglich. Die zu wählende zeitliche Periodizität der Anregung folgt aus dem beobachteten dominanten instationären Verhalten der periodisch ablösenden Zylinderströmung. Die Anregungsstrouhalzahlen sind damit zu $S r_{E}=0,2, S r_{E}=0,3, S r_{E}$ $=0,5$ gegeben. Die Anregungsfrequenz der Lautsprecher wurde dementsprechend für jeden Messpunkt einer Messreihe neu eingestellt, während die spannweitige Periodizität beibehalten wird. Neben dieser Möglichkeit die periodische Ausbildung der Querwirbel zu stören, kann die $\mathrm{KH}$-Instabilität in der abgelösten freien Scherschicht angeregt bzw. angefacht werden. Dazu sind nach Bloor und Gerrard (1963) [13] aber Anregungsfrequenzen notwendig, die etwa eine Größenordnung über der der natürlichen Ablösefrequenz liegen. Aus technischen Gründen wurde für diesen Fall eine konstante Anregungsfrequenz von $f_{E}=806 \mathrm{~Hz}$ benutzt. Sie entspricht mit Variation der Anströmgeschwindigkeit im unterkritischen bis kritischen Bereich bei konstantem Betriebsdruck von etwa $p_{0}=3,5$ bar einer Anregungsstrouhalzahl von 6,0 $>S r_{E}>1,5$. Die spannweitige Wellenlängen der Anregung hingegen resultieren aus den beobachteten spannweitig periodisch großskaligen Längswirbelstrukturen in den Übergangsbereichen. Spannweitige Wellenlängen von $y_{E}=2,1 D, y_{E}=2,9 D, y_{E}=4,4 D$ und $y_{E}=8,9 D$ werden hierfür realisiert. Mit Nutzung des ,Aktiv“-Zylindermodells können wieder die azimutale Druckverteilung in der Symmetrieebene, die spannweitige Druckverteilung stromauf der Anregung bei $\varphi_{P}=55$ Grad und der Basisdruck gemessen werden. Die Störung der Grenzschicht erfolgt wieder nur auf der Oberseite in der Nähe der laminaren Ablösung. Mit der spannweitigen Wellenlänge und der Anregungsstrouhalzahl liegt ein zweidimensionaler Parameterraum vor, der systematisch untersucht wurde.

In Analogie zur Vorgehensweise beim kontinuierlichen Absaugen wird zunächst die akustische Anregung für den spannweitig homogenen Fall angewendet, wobei die Anregungsstrouhalzahl systematisch variiert wird. Im folgenden wird für zwei Anregungsstrouhalzahlen $S r_{E}=0,2$ und $S r_{E}>1,5$ die spannweitige Wellenlänge systematisch verändert. Um insbesondere auch einen Vergleich zu der stationären Anregung durch Absaugen zu ermöglichen, wird für die spannweitigen Wellenlängen $y_{E}=4,4 D$ und $y_{E}=2,2 D$ für verschiedene Anregungsstrouhalzahlen die Wirkung der instationären Anregung diskutiert. Für die hier betrachteten akustischen Anregungen wurde eine Schalldruckamplitude von $L_{p E}=92 \mathrm{~dB}$ benutzt. Andererseits zeigt sich auch bei dieser Anregungsform eine Abhängigkeit von der Größe der Störamplitude. Daher wird abschließend die Effektivität der akustischen Störung in Abhängigkeit von der spannweitigen Wellenlänge, der Strouhalzahl und der Schalldruckamplitude der Anregung erörtert. 


\subsubsection{Spannweitig homogene Anwendung der akustischen Anregung}

In Analogie zur spannweitig homogenen Anwendung des Absaugens wird mit der akustischen Anregung die ablösende Grenzschicht zusätzlich zeitlich periodisch gestört. In Bild 4.13 und Bild 4.14 sind die entsprechenden Druckverteilungen und die spannweitige Verteilung der dominanten Ablösefrequenzen für die angewandten Anregungsstrouhalzahlen dargestellt.
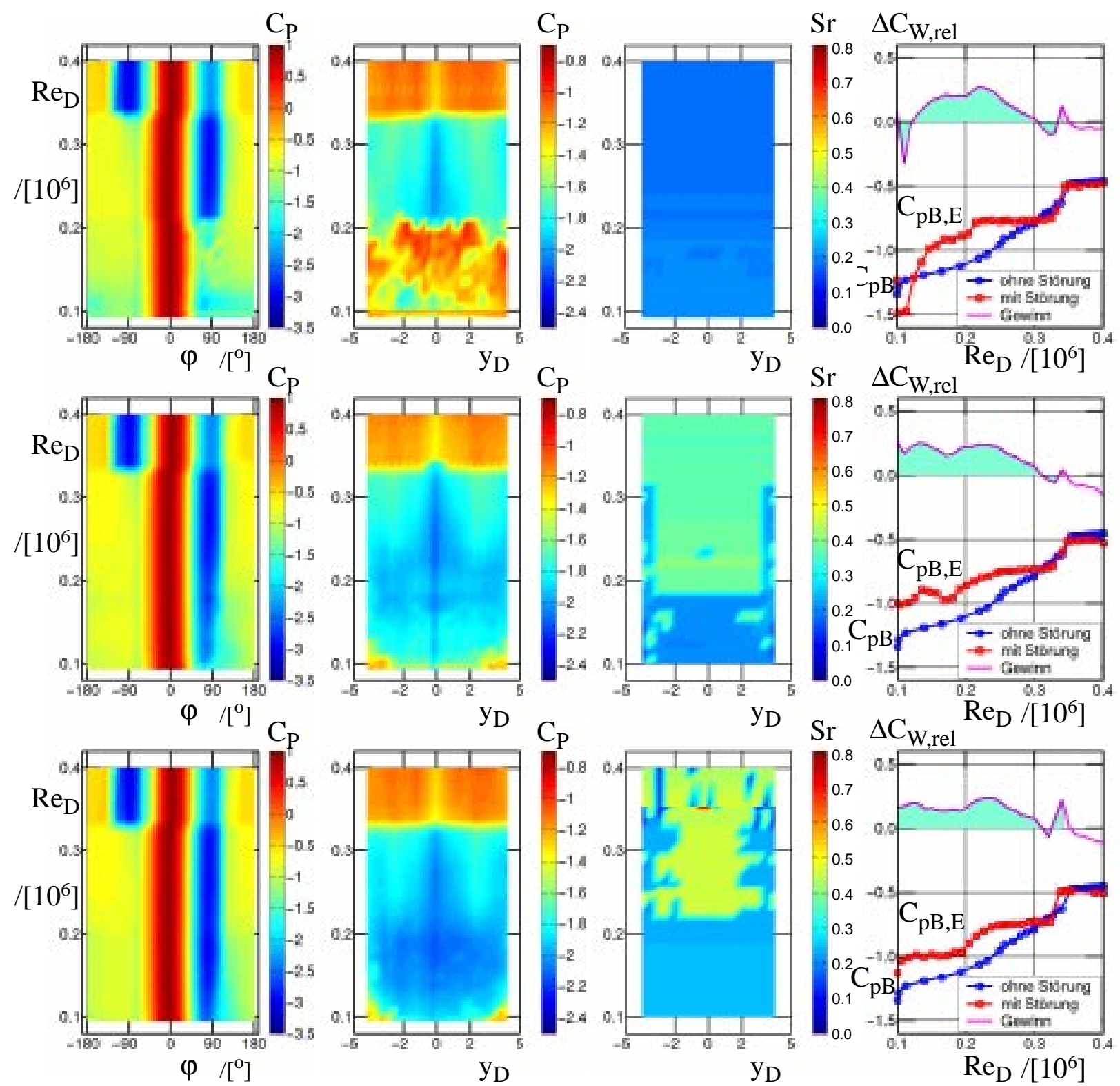

Bild 4.13 Von links nach rechts: Azimutale (1. Spalte), spannweitige (2. Spalte) Druckbeiwertverteilungen und spannweitige Verteilung der Strouhalzahl der dominanten Druckschwankungen (3. Spalte) sowie Basisdruckbeiwertverläufe im Vergleich zur ungestörten abgelösten Zylinderströmung (4. Spalte) bei spannweitig homogener akustischen Anregung mit $L_{p E}=92 \mathrm{~dB}$ und mit Variation der Anregungsstrouhalzahl - von oben nach unten: $S r_{E}=0,2, S r_{E}=0,4$ und $S r_{E}=0,5$ mit mittleren Widerstandsreduktionen von respektive $\overline{\Delta C_{W, \text { rel }}} \approx 10 \%, 16 \%$ und $14 \%$. 
Für alle Anregungsstrouhalzahlen ist zunächst wieder ein ausgeprägter asymmetrisch abgelöster Strömungszustand zumindest ab einer Reynoldszahl von $\operatorname{Re}_{D}=2,0 \times 10^{5}$ zu beobachten und ist vergleichbar mit dem asymmetrischen Zustand im kritischen Bereich der ungestörten Zylinderströmung. Die spannweitige Verteilung von Druckbeiwert und der dominanten Strouhalzahl weisen allerdings ein unterschiedliches Verhalten auf. Die spannweitige Druckverteilung stromauf der Anregung weist bis auf Ausnahme für den Fall bei $S r_{E}=0,2$ einen nahezu homogenen Verlauf im unterkritischen Bereich bis $R e_{D}=2,0^{\times} 10^{5}$ auf, wenn von laminarer symmetrischer Ablösung an den Rändern abgesehen wird. Mit Ausbildung des asymmetrischen Zustandes ist der spannweitige Druckbeiwert zunächst nahezu konstant. Mit zunehmender Reynoldszahl bilden sich ab $R e_{D}=2,5^{\times} 10^{5}$ spannweitig lokale Druckmaxima bei den Spannweiten $y_{D}=-2,5$ und $y_{D}=+2,5$ aus. Diese bleiben bis zum Übergang in den überkritischen Ablösezustand erhalten.
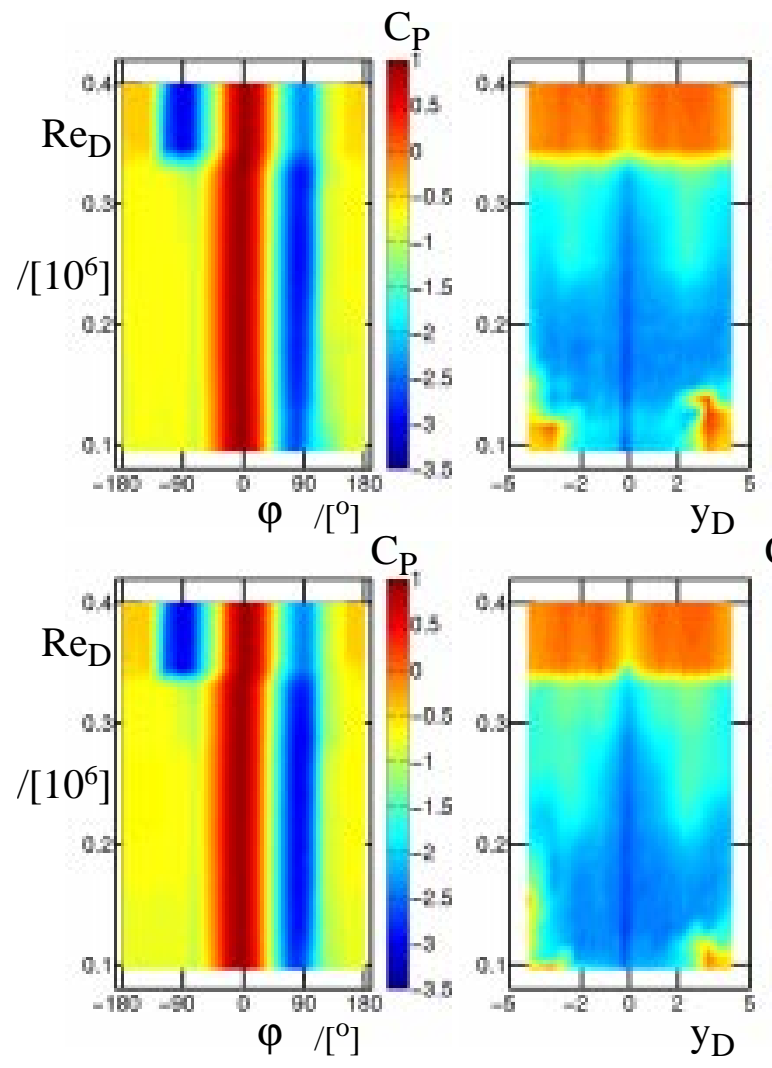

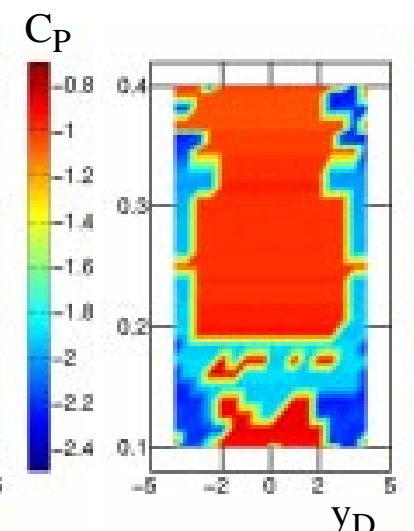

$\mathrm{C}_{\mathrm{P}}$

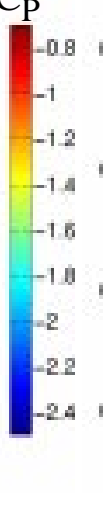

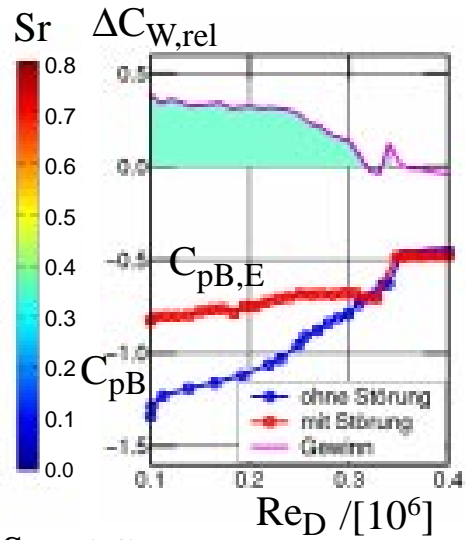

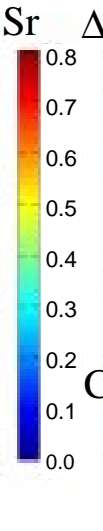

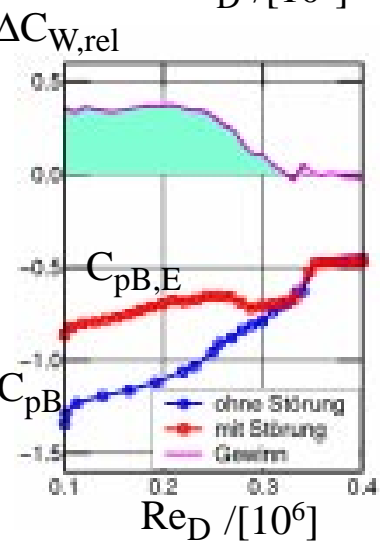

Bild 4.14 Von links nach rechts: Azimutale (1. Spalte), spannweitige (2. Spalte) Druckverteilungen und spannweitige Verteilungen der Strouhalzahl der dominanten Druckschwankungen (3. Spalte) sowie Basisdruckverläufe bei spannweitig homogener akustischen Anregung mit $L_{P E}=92 \mathrm{~dB}$ im Vergleich zu den der ungestört abbgelösten Zylinderströmung (4. Spalte) mit $S r_{E}=0,72$ (obere Reihe) und $S r_{E}>1,50$ (untere Reihe) mit mittleren Widerstandsreduktionen von respektive $\overline{\Delta C_{W, \mathrm{rel}}} \approx 25 \%$ und $\overline{\Delta C_{W, r e l}} \approx 26 \%$

Während im unterkritischen Bereich die Druckschwankungen entsprechend der laminaren symmetrischen Ablösung mit $S r=0,2$ dominieren, ist im kritischen bis überkritischen Bereich das 
instationäre Verhalten genauer zu betrachten. Die stromauf der Anregung gemessenen Druckschwankungen werden maßgeblich durch die zeitlich periodische Störung geprägt. Somit weist die spannweitige Verteilung der Strouhalzahl auch diejenige auf, mit der angeregt wurde. Lediglich an den lateralen Enden wird dieses instationäre Verhalten durch niederfrequente Fluktuationen beeinträchtigt. Eine Ausnahme stellt lediglich der Fall mit „S $S r_{E}>1,5^{\text {“ dar. Hier }}$ dominieren beginnend im unterkritischen bis zum Übergang in den überkritischen Bereich Druckschwankungen mit etwa $S r=0,3$. Auch im Fall für , $S r_{E}=0,72$ “ ist zu erkennen, dass zwar im inneren spannweitigen Bereich Druckschwankungen entsprechend zu $\mathrm{Sr}=0,72$ die größte Amplitude besitzen, aber zu den lateralen Enden des Zylinders hin ebenfalls Schwankungen mit $S r=0,3$ zunehmen. Bei den Fällen mit geringerer Anregungsstrouhalzahl sind an den Rändern hingegen eher Schwankungen mit $S r=0,2$ festzustellen. Das instationäre Verhalten der Grenzschicht ist in Abhängigkeit von der Anregungsstrouhalzahl also unterschiedlich, so dass dieses Verhalten auch auf unterschiedliche Anregungsmechanismen basieren könnte. Der inhomogene Verlauf der spannweitigen Druckverteilungen im unterkritischen Bereich für,$S r_{E}=$ 0,2 " könnte darauf zurückgeführt werden, dass die Anregungsfrequenz nicht exakt auf die Frequenz der symmetrischen Ablösung passte. Damit erfolgt nur bedingt ein Einkoppeln der periodischen Störung in die periodisch ablösende Zylinderströmung. In diesem Zusammenhang ist auch festzustellen, dass die mittlere Widerstandsreduktion für den unterkritischen und kritischen Bereich für die höheren Anregungsstrouhalzahlen größer als die mit $S r_{E}<=0,5$ ist. Dieser Unterschied beruht maßgeblich auf eine Verbesserung des Basisdruckbeiwertverlaufes in dem unterkitischen Bereich von $\operatorname{Re}_{D}=1,0^{\times} 10^{5}$ bis $R e_{D}=2,0^{\times} 10^{5}$. Mit größerer Anregungsstrouhalzahl strebt der Basisdruckbeiwert auch hier einem Wert von $C_{p B}=-0,6 \mathrm{zu}$.

Für die Abhängigkeit der Widertstandsreduktion von der Anregungsstrouhalzahl lassen sich zwei Erklärungen angeben. Die eine folgert aus dem Ergebnis für , $S r_{E}=0,2$ “. Mit zunehmender Anregungsstrouhalzahl für größere Werte als $S r_{E}=0,2$ ist die relative periodische Anregung bezüglich der Strouhalzahl der ungestörten periodisch abgelösten Zylinderströmung im unterkritischen Bereich mit $S r=0,2$ größer als Eins. Während sie bei $S r_{E}=0,5$ noch den Faktor 2,5 annimmt, beträgt sie im Mittel für 6,0 $>S r_{E}>1,5$ bereits etwa 18. Das bedeutet, dass die Störung der ablösenden Grenzschicht im Mittel 18 mal häufiger erfolgt. D.h., es kommt zu einem zunehmenden Eintrag von Störungen in die Grenzschicht, die daraufhin turbulent wird und so wieder anlegt, um weiter stromab turbulent abzulösen. Eine andere Erklärung beruht ähnlich wie bei der Argumentation beim kontinuierlichen Absaugen auf der These, dass sich durch die periodische Anregung durch die Bohrungen der Kammern in der ablösenden Grenzschicht spannweitig kleinskalige Längswirbelstrukturen ausbilden. Mit der Stromabentwicklung und Wechselwirkung dieser Längswirbel wird die Grenzschicht instabil. Bei Erreichen einer, mit dieser eingebrachten Störung kritischen Reynoldszahl erfolgt der Übergang in den asymmetrisch abgelösten Zustand. D.h., die ablösende Grenzschicht wird mit Entstehung der Längswirbel turbulent, legt am Zylinder wieder an, um erst weiter stromab turbulent abzulösen. Mit der Erhöhung der Anregungsstrouhalzahl wird zudem der angefachte Bereich der $K H$-Instabilität erreicht. Die sich in der ablösenden Grenzschicht konvektiv ausbreitenden Längswirbel werden somit in der instabilen Scherschicht noch weiter verstärkt. Aufgrund dieser Wechselwirkung scheint mit zunehmender Anregungsstrouhalzahl der Übergang in den asymmetrisch abgelösten Zustand zu immer kleineren Reynoldszahlen verschoben zu werden. 


\subsubsection{Spannweitig periodische Anwendung der akustischen Anregung}

Mit der spannweitig periodischen Anwendung der akustischen Anregung wird die spannweitige Wellenlänge bei konstanter Anregungsstrouhalzahl variiert. Die Frage ist, ob im Vergleich zur spannweitig periodischen Anwendung des kontinuierlichen Absaugens die Instationarität bei der hier betrachteten Anregungsform einen anderen Effekt zeigt bzw. inwiefern die zusätzlich zeitlich periodisch eingebrachte Störung das Ablöseverhalten der Zylinderströmung beeinflusst.
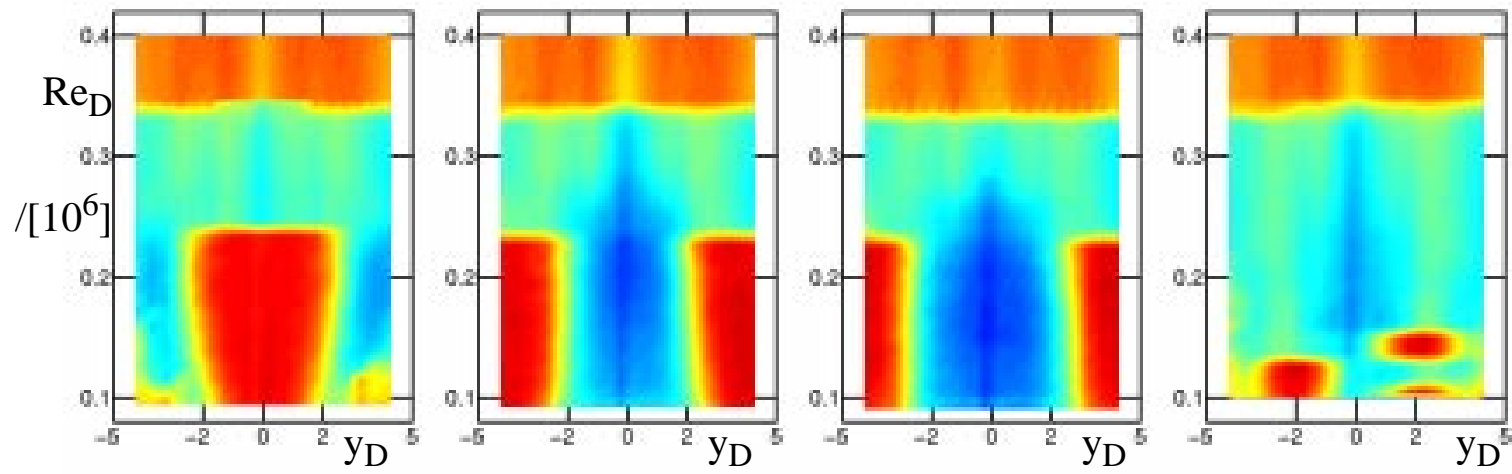

$\mathrm{C}_{\mathrm{P}}$
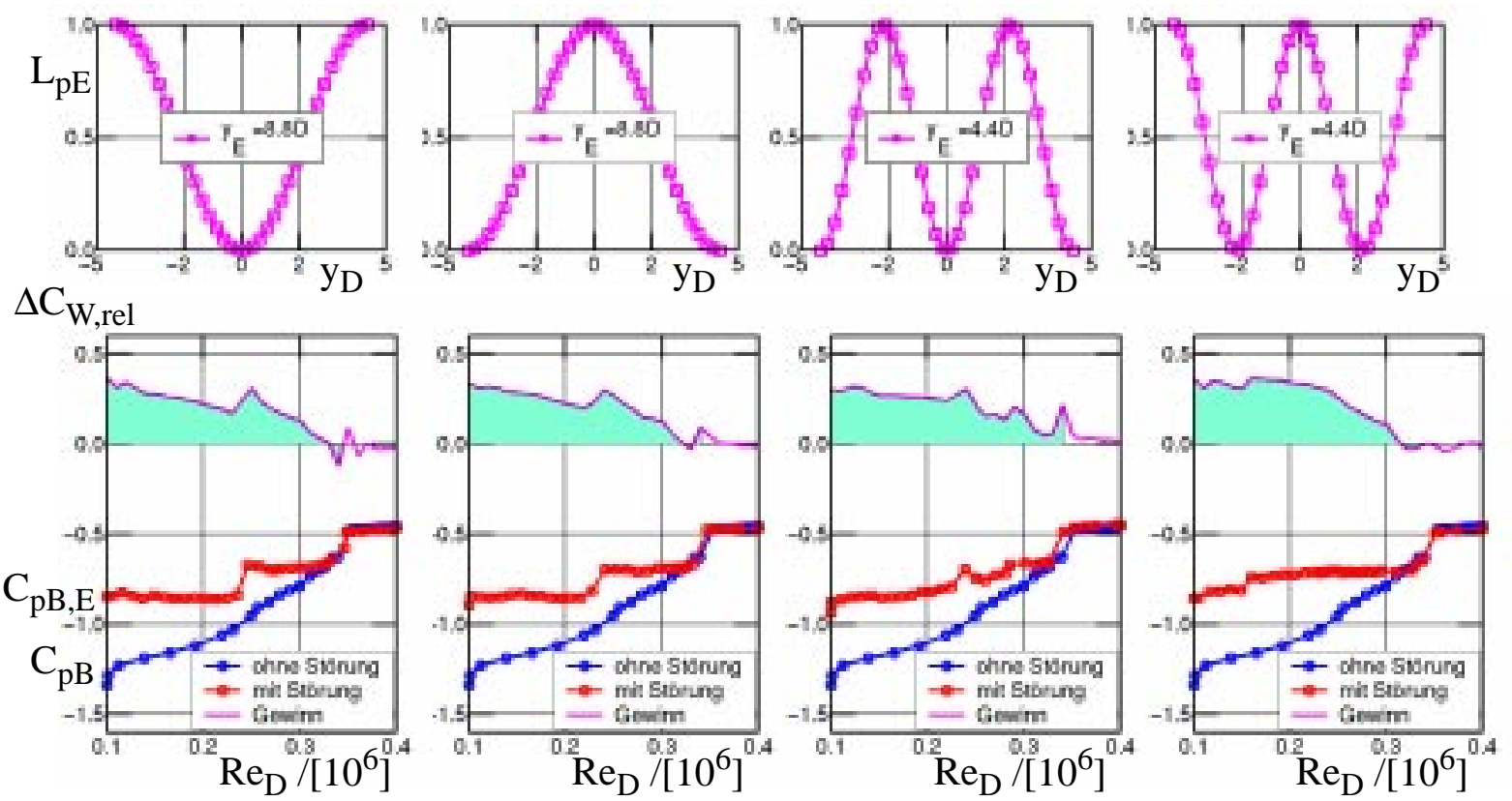

Bild 4.15 Von links nach rechts: Akustische Anregung mit $L_{p E}=92 \mathrm{~dB}$ und $S r_{E}>1,5$ bei Variation der spannweitigen Wellenlänge $y_{E}=8,9 \mathrm{D}(\mathrm{min})$ mit $\Delta C_{W}=21 \% ; y_{E}=8,9 \mathrm{D}$ (max) mit $\overline{\Delta C_{W, \text { rel }}} \approx 21 \% ; y_{E}=4,4 D(\max )$ mit $\overline{\Delta C_{W, \text { rel }}} \approx 25 \% ; y_{E}=4,4 D(\min )$ mit $\overline{\Delta C_{W, \text { rel }}} \approx 24 \%$. Obere Reihe: Spannweitige Druckverteilungen. Mittlere Reihe: Spannweitige Störamplitudenverteilung der akustischen Anregung. Untere Reihe: Basisdruckbeiwertverlauf der gestörten im Vergleich mit der ungestörten abgelösten Zylinderströmung und relative Widerstandsreduktion. 
Zur Beantwortung dieser Frage wurde die spannweitige Wellenlänge für eine Anregungsstrouhalzahl mit $S r_{E}>1,5$ variiert, da hiermit bei der spannweitig homogenen Anregung der größte Effekt in der Widerstandsreduktion erzielt wurde. Weiterhin werden aus Symmetriegründen die beiden möglichen Konfigurationen zu jeder spannweitigen Wellenlänge umgesetzt. D.h., erfolgt in der Symmetrieebene des Zylinders lokal die Einbringung einer Störung wird dies mit „max“, andernfalls mit ,min“ gekennzeichnet. Die spannweitige Störamplitudenverteilung ist in jedem Fall aber symmetrisch zur Zylindermitte. Wie bereits bei der spannweitig homogenen akustischen Anregung gesehen wurde, kann die azimutale Druckverteilung nur in der Symmetrieebene des Zylinders Auskunft darüber geben, ob sich der asymmetrisch abgelöste Strömungszustand ausgebildet hat. Da nun die spannweitige Wellenlänge und die räumliche Phasenlage der Störamplitudenfunktion verändert wird, ist die azimutale Druckverteilung für vergleichende Betrachtungen nicht nutzbar.

In Bild 4.15 und Bild 4.16 sind die spannweitigen Druckverteilungen und die Basisdruckverläufe für die jeweiligen spannweitig periodischen akustischen Anregungen dargestellt. Bereits ab $R e_{D}=1,0^{\times} 10^{5}$ im unterkritischen Bereich besitzt auch die spannweitige Druckverteilung eine nahezu periodische Verteilung. Dabei tritt spannweitig lokal ein minimaler Druckbeiwert im allgemeinen dort auf, wo die spannweitige Störamplitudenvertielung ein Maximum hat. Lediglich für die spannweitige Anregung mit der kleinsten Wellenlänge von $y_{E}=2,2 D$ bildet die spannweitige Druckverteilung keine vier Maxima aus. Andererseits wechseln sich auch für diesen Fall bei Reynoldszahlen kurz vor der „Transition A“ spannweitige Bereiche mit maximalem und minimalem Druckbeiwert mit bestimmten Längen ab. Werden die spannweitigen Längen mit lokal minimalem Durckbeiwert ausgemessen, so ergeben sich ähnliche Längen wie beim spannweitig periodischen Absaugen zu: $y_{L}=1,2 D ; 1,7 D ; 2,5 D$ und 3,3D ( $s$. A.5 Re-gemittelte Widerstandsreduktionen bei aktiver Beeinflussung). Der Übergang selbst findet für alle hier betrachteten Fälle bei unterschiedlichen Reynoldszahlen statt. Im allgemeien kann aber gesagt werden, dass er einem Reynoldszahlbereich zwischen $R e_{D}=2,3 \times 10^{5}$ und $R e_{D}=3,0 \times 10^{5}$ erfolgt. Darüberhinaus ist die spannweitige Druckverteilung zu der Symmetrieebene des Zylinders weitestgehend symmetrisch, was aufgrund der Symmetrie der künstlichen Anregung auch erwartet wird.

Eine Ausnahme stellt lediglich die Anregung mit der spannweitigen Wellenlänge von $y_{E}=4,4 D$ ( $\max$ ) dar. Hier ist der Übergang zum asymmetrischen spannweitig homogen abgelösten $\mathrm{Zu}-$ stand bereits bei einer Reynoldszahl von $R e_{D}=1,5^{\times} 10^{5} \mathrm{zu}$ beobachten. Dies findet seinen Niederschlag in einer mittleren Widerstandsreduktion von $\overline{\Delta C_{W, \text { rel }}} \approx 24 \%$. Der Basisdruckbeiwert bleibt im unterkritischen bis kritischen Reynoldszahlbereich bei $C_{p B}=-0,65$ nahezu konstant. Die aus der spannweitigen Druckverteilung ablesbare typische Länge laminarer Ablösung beträgt hier $y_{L}=2,5 D$. Weiterhin tritt bei dieser spannweitig periodischen Anregung einmalig ein spannweitig asymmetrischer Zustand auf. Demnach muss die sich ablösende Grenzschicht auch instabil gegenüber spannweitig asymmetrischen Störungen sein. Da die Symmetrie der Ablösestruktur erhalten bleiben muss, ist ein einseitig spannweitig und azimutal asymmetrisch abgelöster Strömungszustand unwahrscheinlich bzw. instabil und ist unter topologischer Betrachtung an einem Zylinder nicht möglich (Dallmann und Schewe 1987 [20]). Daher müsste auf der Unterseite des Zylinders die spiegelbildliche spannweitige Druckverteilung vorherrschen. Ein Beweis dafür ist im Rahmen der an diesem Zylindermodell instrumentierten Sensoren nicht gegeben. Später wird in 4.3.4 Einfluss der Variation der Schalldruckpegelamplitude noch genauer auf dieses Ablöseverhalten eingegangen.

Markant für die hier betrachteten spannweitig periodischen Anregungen ist, dass sie nur anfäng- 
lich eine bestimmte mehr oder weniger spannweitig periodische Druckverteilung bewirken, die dann ab einer kritischen Reynoldszahl in eine spannweitig homogene Druckverteilung übergeht. Diese Druckverteilung entspricht die der asymmetrisch abgelösten ungestörten Zylinderströmung im kritischen Bereich. Bemerkenswert dabei ist das Auftreten nur bestimmter spannweitiger Längen, die mit spannweitig periodisch ausgebildeten Länswirbelstrukturen in Verbindung gebracht werden könnten.
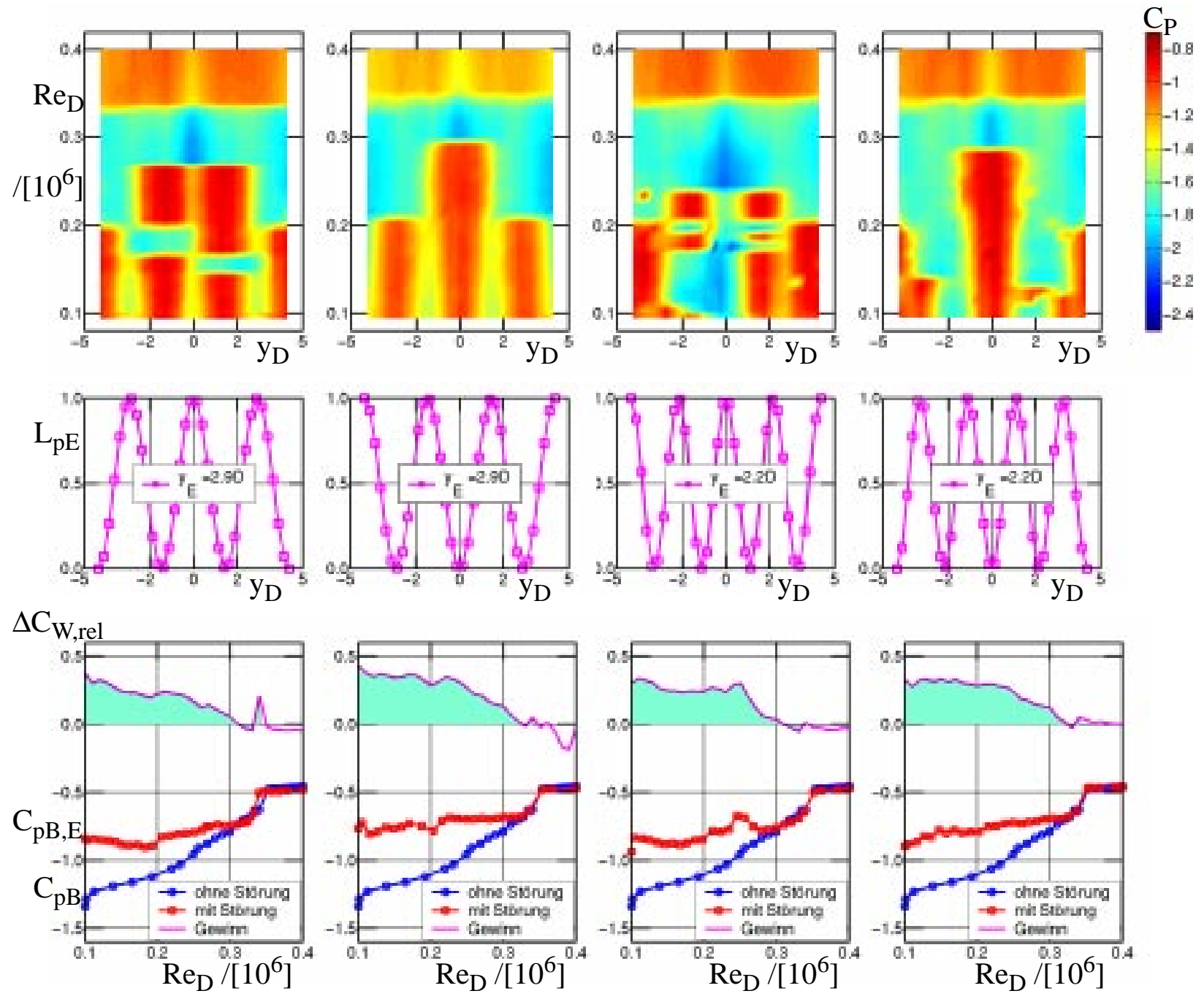

Bild 4.16 Von links nach rechts: Akustische Anregung mit $L_{p E}=92 \mathrm{~dB}$ und $S r_{E}>1,5$ bei Variation der spannweitigen Wellenlänge $y_{E}=2,9 D(\max )$ mit $\overline{\Delta C_{W, r e l}} \approx 18 \% ; y_{E}=$ $2,9 D(\min )$ mit $\overline{\Delta C_{W, \text { rel }}} \approx 26 \% ; y_{E}=2,1 D($ max $)$ mit $\overline{\Delta C_{W, \text { rel }}} \approx 19 \% ; y_{E}=2,1 D$ (min) mit $\overline{\Delta C_{W, \text { rel }}} \approx 22 \%$. Obere Reihe: Spannweitige Druckverteilungen. Mittlere Reihe: Spannweitige Störamplitudenverteilung der akustischen Anregung. Untere Reihe: Basisdruckbeiwertverlauf der gestörten im Vergleich mit der ungestörten abgelösten Zylinderströmung und relative Widerstandsreduktion.

Durch die spannweitig verteilte Störung der Grenzschicht könnten spannweitig lokal aufgrund des instationären Charakters der Anregung Längswirbelstrukturen entstehen, die die Grenz- 
schicht lokal instabil werden lassen. Weiterhin liegt die Anregungsfrequenz in dem Bereich, wo $\mathrm{KH}$-Wellen in der abgelösten Scherschicht angeregt bzw. angefacht werden. Da diese bei diesen großen Reynoldszahlen selbst schon instabil sind, würden diese selbst in spannweitig periodisch angeordneten Längswirbelpaaren übergehen. Aufgrund des globalen Charakters der Ablösung würde diese spannweitig lokale und einseitige Änderung des Scherschichtzustandes das globale Verhalten der Zylinderströmung ändern. Die symmetrisch abgelöste Mode müsste instabil werden. Wird dann die mit der instabilen Scherschichtentwicklung zugehörige kritische Reynoldszahl erreicht, erfolgt der globale Übergang vom symmetrisch zum asymmetrisch abgelösten Zustand. Dabei scheint die globale symmetrische Mode stärker instabil gegenüber spannweitig asymmetrischen abgelösten Zuständen zu sein, da - wie im Fall mit , $y_{E}=4,4 D^{\prime \prime}$ gesehen wurde, der Übergang bei einer deutlich kleineren Reynoldszahl stattfindet, als in den Fällen, wo vor dem Übergang eine spannweitig symmetrische Druckverteilung beobachtet wird.

\subsubsection{Zeitlich periodische Anwendung der akustischen Anregung}

Mit der spannweitig periodischen Anregung mit $S r_{E}>1,5$ hatte sich gezeigt, dass insbesondere bei einer spannweitigen Wellenlänge von $y_{E}=4,4 D$ und mit maximaler Anregung in der Symmetrieebene des Zylinders eine beträchtliche Widerstandsreduktion erreicht wurde. Dies wurde insbesondere auf das globale Instabilitätsverhalten zurückgeführt. Um zu klären, ob nur die spannweitige Periodizität der zeitlich harmonischen Störung ein Auswahlkriterium ist, um besonders große Widerstandsreduktionen zu erreichen, wird für diese spannweitige Wellenlänge die Anregungsstrouhalzahl von $\mathrm{Sr}_{E}=0,2$ bis $S r_{E}>1,5$ variiert.

Bei der spannweitig homogenen instationären Störung wurde bereits mit Variation der Anregungsstrouhalzahl festgestellt, dass insbesondere die hochfrequente Anregung die größte Widerstandsreduktion liefert. Auch für die spannweitig periodische Anregung wird dieses Ergebnis vermutet. Sollte sich allerdings eine von der Anregungsstrouhalzahl nahezu unabhängige Effektivität in der Widerstandsreduktion zeigen, so könnte daraus geschlossen werden, dass der Übergang vom symmetrisch zum asymmetrisch abgelösten Strömungszustand zwar eine zeitlich harmonische Störung benötigt, die dominierende Instabilität aber auf einer spannweitig periodischen großskaligen Instabilität beruht.

Neben spannweitigen Druckverteilungen bei $\varphi_{P}=55$ Grad und Basisdruckverläufe werden auch spannweitige Verteilungen der dominanten Strouhalzahl der Wirbelablösung in Bild 4.17 dargestellt. Anhand der Basisdruckverläufe wird sofort erkannt, dass mit zunehmender Anregungsstrouhalzahl auch eine zunehmende mittlere Widerstandsreduktion erreicht wird, wobei diese bereits bei einer Anregung mit $S r_{E}=0,5$ schon vergleichbar zu der mit $S r_{E}>1,5$ ist. Bis auf den bereits bekannten Fall mit der Anregungsstrouhalzahl $S r_{E}>1,5$, ist bei den anderen Fällen wieder eine spannweitig symmetrische Druckverteilung vor der „Transition A“ festzustellen. Darüberhinaus vollzieht sich der Übergang für diese Fälle nahezu bei der gleichen Reynoldszahl von etwa $R e_{k r i t, A}=2,4 \times 10^{5}$. Die spannweitige Verteilung der dominanten Strouhalzahl liefert allerdings noch tiefergehende Einsichten in das Instabilitätsverhalten der angeregten Grenzschicht für Anregungsstrouhalzahlen kleiner $S r_{E} \leq 0,72$.

Für $S r_{E} \leq 0,72$ kann bei kleinen Reynoldszahlen im unterkritischen Bereich von $R e_{D}=1,0^{\times} 10^{5}$ bis $\operatorname{Re}_{D}=1,5^{\times} 10^{5}$ eine mehr oder weniger spannweitig periodische Verteilung der dominanten Strouhalzahl, die mit der Anregungsstrouhalzahl übereinstimmt, ermittelt werden. Sie treten dort auf, wo die spannweitige Verteilung der Störamplitude ihre Maxima hat. Damit ergibt sich ein spannweitige Korrelation des zeitlich gemittelten und instationären Verhaltens der Grenz- 
schicht für diesen Reynoldszahlausschnitt. Mit weiter zunehmender Reynoldszahl dominieren Druckschwankungen zu $S r=0,2$ nahezu spannweitig homogen.
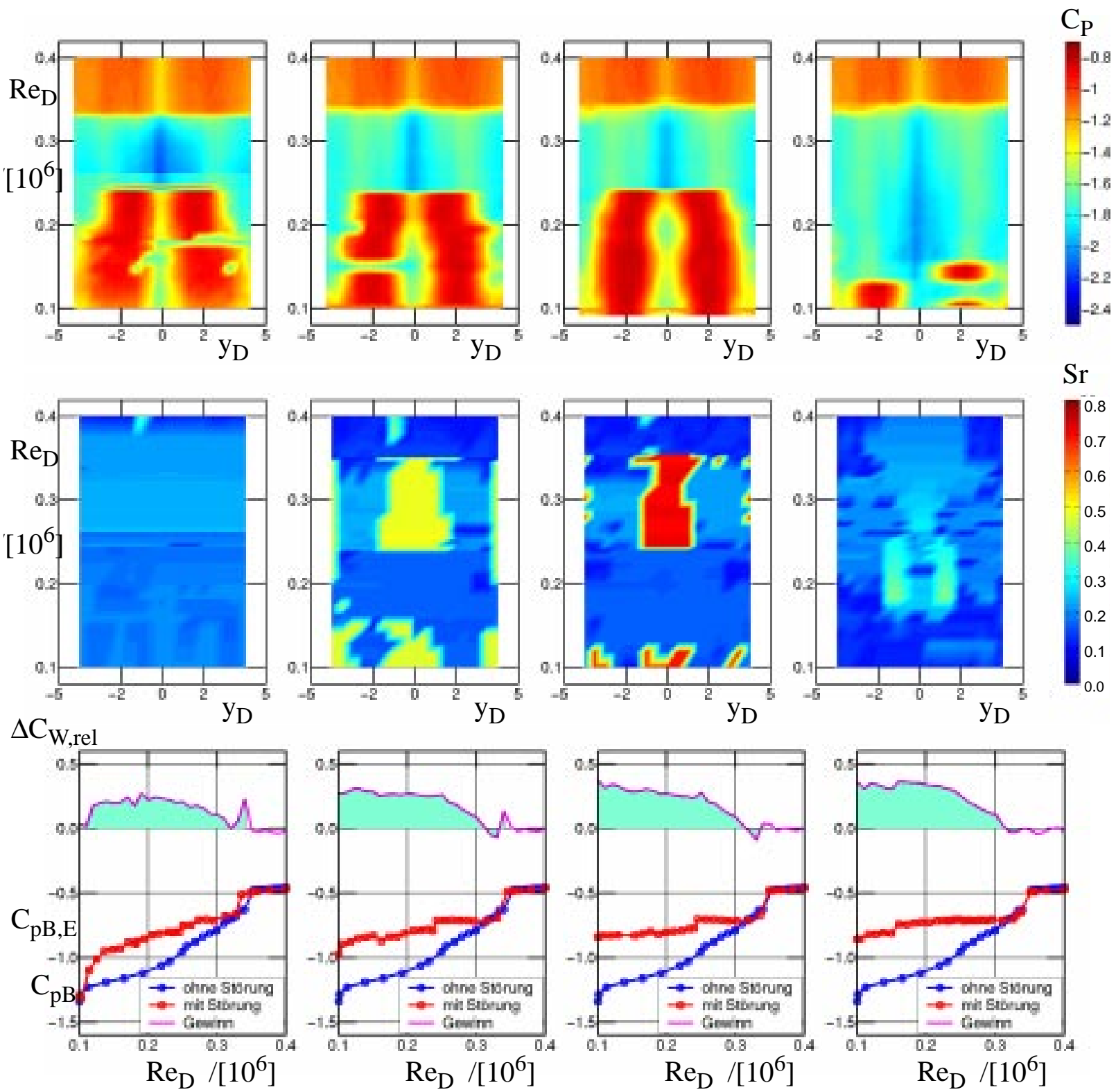

Bild 4.17 Spannweitige Verteilungen des Druckbeiwertes (obere Reihe) und der Strouhalzahl der dominanten periodischen Schwankungen (mittlere Reihe) sowie Basisdruckbeiwertverläufe im unterkritischen bis anfänglich überkritischen Bereich imVergleich mit der ungestörten abgelösten Zylinderströmung (untere Reihe) bei Anwendung der akustische Anregung mit $L_{p E}=92 \mathrm{~dB}$ und einer spannweitigen Wellenlänge von $y_{E}=4,4 D$ (max) und Variation der Anregungsstrouhalzahl. Von links nach rechts: $S r_{E}=0,20 ; 0,50 ; 0,72$ und $S r_{E}>1,50$. Die dabei erreichten mittleren Widerstandsreduktionen betragen respektiv $\overline{\Delta C_{W, \text { rel }}} \approx 21 \%, 21 \%, 21 \%$ und $24 \%$. 
Mit dem Übergang zum asymmetrisch abgelösten Zustand wird ein vielfältigeres Verhalten festgestellt. In dem inneren spannweitigen Bereich von $y_{D}=-2,0$ bis $y_{D}=+2,0$ und an teilweise an den seitlichen Begrenzungen sind Druckschwankungen zu der Strouhalzahl bestimmend, mit der auch angeregt wird. An den verbleibenden Spannweiten dominieren Druckschwankungen $\mathrm{zu} S r=0,3$, wie sie auch bei der ungestörten asymmetrisch abgelösten Zylinderströmung beobachtet werden. Für die Anregungsstrouhalzahl mit $S r_{E}>1,5$ hingegen wird das spannweitige instationäre Ablöseverhalten zumeist von periodischen Schwankungen zu der Strouhalzahl $\mathrm{Sr}$ $=0,3$ beherrscht. Insbesondere ab einer Reynoldszahl von $\operatorname{Re}_{D}=1,5^{\times} 10^{5}$ bis $\operatorname{Re}_{D}=3,3^{\times} 10^{5}$ sind nahezu über die gesamte Spannweite dominierend. Lediglich im Reynoldszahlbereich von $R e_{D}$ $=1,8^{\times} 10^{5}$ bis $\operatorname{Re}_{D}=2,2^{\times} 10^{5}$ sind Schwankungen mit einer Strouhalzahl von $S r=0,5$ an zwei spannweitigen Positionen bei $y_{D}=-1,4$ und $y_{D}=+1,4$ bestimmend.

Mit diesen Ergebnissen folgt, dass bei geeigneter Kopplung von spannweitig und zeitlich periodischer Anregung größere Widerstandsreduktionen schon bei kleineren Anregungsstrouhalzahlen möglich sind. Eine notwendige Bedingung dafür scheint in der Wahl einer passenden spannweitigen Wellenlänge der Anregung zu sein, die inhärent vorhandene spannweitig periodische Wirbelstrukturen anregen kann. Die symmetrisch spannweitig homogen ablösende Grenzschicht scheint gegenüber spannweitig periodischen Störungen instabil zu sein. Ablesbar an den spannweitig periodischen Druckverteilungen könnten die Bereiche mit lokal minimalem Druckbeiwert mit der Ausbildung von Längswirbelstrukturen in der Grenzschicht verknüpft sein. Während die spannweitig periodische Störung das Grundströmungsprofil der Grenzschicht spannweitig deformiert, sorgt die zeitlich harmonische Anregung für die Produktion von kleinskaliger Turbulenz, die in der Generierung von zunächst kleinskaligen Längswirbeln mündet. Diese Wirbelproduktion nimmt mit Erhöhung der Anregungsstrouhalzahl zu und wird scheinbar begünstigt durch die instabile abgelöste Scherschicht. Mit dem konvektivem Transport und der Verschmelzung benachbarter Längswirbel bilden sich in der instabilen abgelösten Scherschicht zunehmed spannweitig großskalige Längswirbelstrukturen aus. Mit zunehmender Reynoldszahl wandern diese Längswirbelstrukturen stromauf und erreichen bei einer bestimmten kritischen Reynoldszahl den Ort der Ablösung. Damit wird der global symmetrisch abgelöste Zustand mit ausgebildeter spannweitig periodischer Wirbelstruktur instabil. Mit der „Transition A“ geht die Ablösung in den global asymmetrischen Zustand über. Dieser ist zunächst stabil und bildet sich daher kohärent über der gesamten Spannweite aus. Er ist gekennzeichnet durch die Entstehung einer transitionellen Ablöseblase auf der Seite des Zylinders, wo die Anregung stattfindet.

Mit weiter zunehmender Reynoldszahl ist wieder anhand der spannweitigen Druckverteilung zu erkennen, dass die transitionelle Ablöseblase instabil gegenüber spannweitig periodischen Störungen wird. Trotzdem wird die „Transition B“ nicht zu kleineren Reynoldszahlen hin verschoben. Eine mögliche naheliegende Erkärung ist die, dass mit der einseitigen Anregung nur lokal das Instabilitätsverhalten der ablösenden Grenzschicht bzw. der transitionellen Ablöseblase beeinflusst wird. Soll der globale asymmetrische Zustand in der Weise gestört werden, dass dieser gegenüber dem globalen symmetrischen Zustand des überkritischen Bereiches instabil wird, müsste die Grenzschicht auch auf der anderen Seite des Zylinders angeregt werden.

\subsubsection{Einfluss der Variation der Schalldruckpegelamplitude}

Bei der Anregung mit $y_{E}=4,4 D$ und $S r_{E}>1,5$ wurde vor dem Übergang zum globalen asymmetrisch abgelösten Zustand eine spannweitig asymmetrische Druckverteilung stromauf der 
Anregung beobachtet. Ein derartiges Verhalten der Grenzschicht wurde nur mit diesen Parametereinstellungen festgestellt.
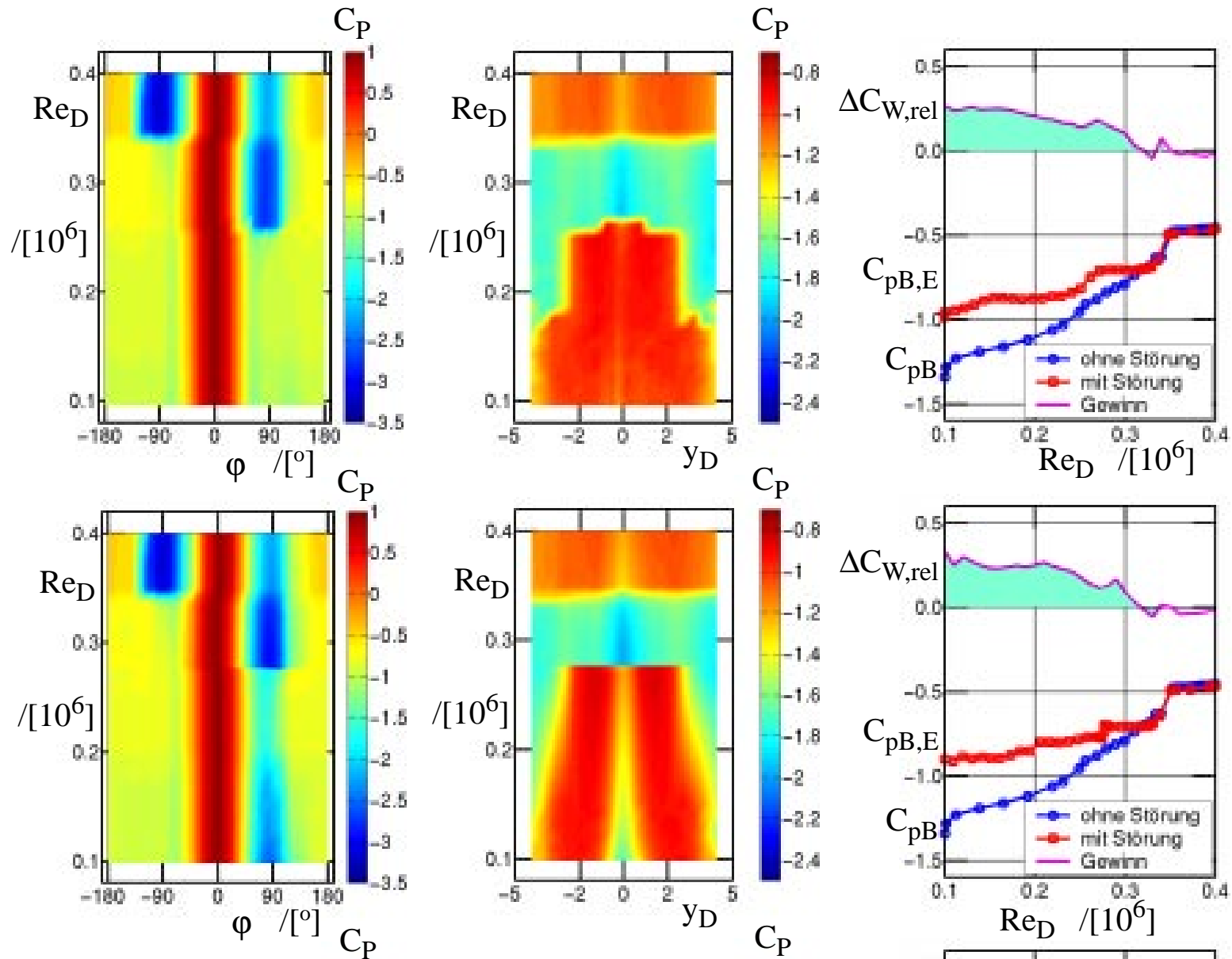

$\mathrm{C}_{\mathrm{P}}$
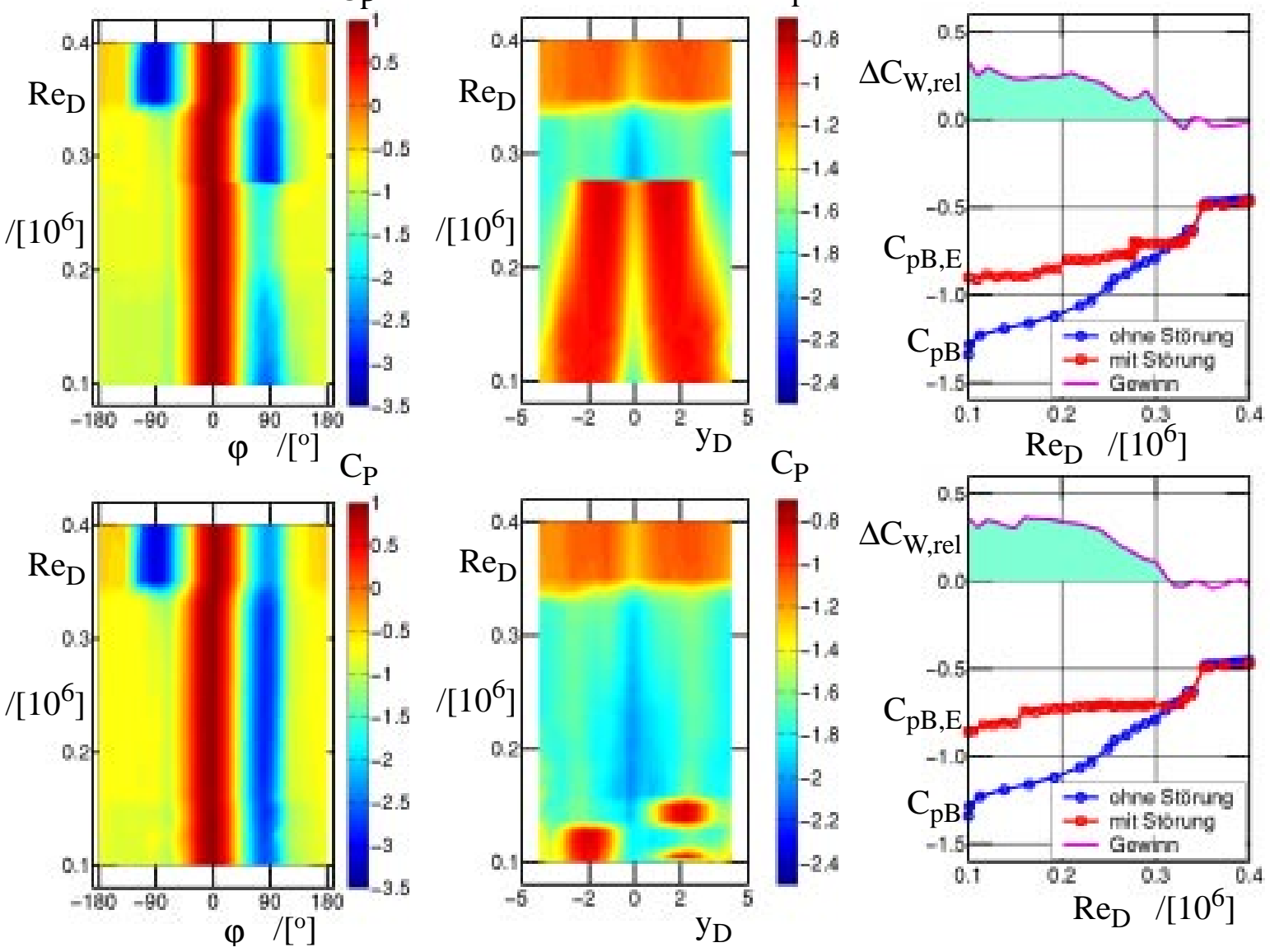

Bild 4.18 Variation der Schalldruckamplitude bei Anregung mit $y_{E}=4,4 D(\max )$ und $S r_{E}>1,5$ - von oben nach unten: $L_{p E}=80 \mathrm{~dB}$ mit $\overline{\Delta C_{W, \text { rel }}} \approx 17 \%, L_{p E}=86 \mathrm{~dB}$ mit $\overline{\Delta C_{W, r e l}} \approx 19 \%$ und $L_{p E}=92 \mathrm{~dB}$ mit $\overline{\Delta C_{W, r e l}} \approx 24 \%$. Von links nach rechts: Azimutale und spannweitige Druckverteilungen sowie die Basisdruckbeiwertverläufe der gestörten im Vergleich zu der ungestörten Zylinderströmung mit relativer Widerstandsreduktion. 
Es liegt nahe, dass aufgrund der hohen Störamplitude in Verbindung mit dieser passenden spannweitig periodischen Anregung, die Grenzschicht eher instabil gegenüber der spannweitig asymmetrischen Mode ist. Mit einer Verringerung der Störamplitude müsste also wieder eine spannweitig symmetrische Druckverteilung zu messen sein. In Bild 4.18 sind die azimutalen und spannweitigen Druckverteilungen und Basisdruckverläufe mit den Schalldruckamplituden von $L_{p E}=80 \mathrm{~dB}, L_{p E}=86 \mathrm{~dB}$ und $L_{p E}=92 \mathrm{~dB}$ dargestellt. In der Tat werden mit Änderung der Schalldruckamplitude unterschiedliche spannweitige Druckverteilungen vor der „Transition $A^{\text {“ }}$ erhalten. Für die Fälle mit geringerer Schalldruckamplitude als $L_{p E} \leq 86 \mathrm{~dB}$ bildet sich eine spannweitig symmetrische Druckverteilung aus. Insbesondere mit,$L_{p E}=86 \mathrm{~dB}$ “ stimmt die spannweitige Verteilung der Druckminima stimmt gut mit den Maxima der spannweitigen Störamplitudenverteilung überein. Für , $L_{p E}=80 \mathrm{~dB}$ “ wiederum sind lediglich an den seitlichen Begrenzungen des Zylinders entsprechende Druckminima ausgebildet. Mit Zunahme der Störamplitude kann der Grenzschicht also effektiver eine spannweitig periodische Störung aufgeprägt werden. Damit verbunden ist die Verschiebung der „Transition A“ zu kleineren Reynoldszahlen, was zu einer zunehmenden mittleren Widerstandsreduktion führt. Umgekehrt könnte auch gesagt werden, dass die Grenzschicht es vermag, Störungen bestimmter Amplitude noch auszugleichen. D.h., die in der Grenzschicht induzierten kleinskaligen Wirbelstrukturen dissipieren und die intermittente Grenzschicht relaminarisiert. Daher wird bei der Anregung mit ${ }, L_{p E}=80 \mathrm{~dB}$ “ in der Symmetrieebene des Zylinders ein Druckbeiwert gemessen, der dem unterkritischen Zustand entspricht, obwohl hier maximale Anregung erfolgt. Weiterhin wird die Zylindergrenzschicht an den Rändern durch die Ausbildung eines Hufeisenwirbel an jeder Seitenwand der Messstrecke beeinträchtigt. Darüberhinaus nimmt mit zunehmender Reynoldszahl die relative Störamplitude zur Anströmgeschwindigkeit ab, so dass für , $L_{p E}=86 \mathrm{~dB}$ " gleich zwei Effekte eintreten. Einerseits kommt es trotz maximaler Anregung in der Symmetrieebene zur Relaminarisierung, anderseits zu einem Anwachsen natürlicher Störungen an den Rändern. Die Folge ist, dass mit zunehmender Reynoldszahl die laminare Grenzschicht in den inneren spannweitigen Bereich des Zylinders gedrängt wird.

Die spannweitig asymmetrische Druckverteilung vor der „Transition A“ wird bei akustischer Anregung tatsächlich nur für $y_{E}=4,4 D, S r_{E}>1,5$ und $L_{p E}=92 \mathrm{~dB}$ beobachtet. Die damit verbundene spannweitig asymmetrische Zustand würde sich dadurch auszeichnen, dass die asymmetrische Mode bei ausreichend großer Anregungsamplitude gegenüber der symmetrischen Mode einer stärkeren Anfachung unterliegt. 


\section{Zusammenfassung}

Die vorliegende Arbeit beschäftigt sich mit der experimentellen Untersuchung des laminar-turbulenten Überganges der zweidimensionalen, inkompressiblen ablösenden Grenzschicht am querangeströmten Kreiszylinder. Zur eindeutigen Beschreibung der von der Reynoldszahl abhängigen Vorgänge und Änderungen der abgelösten Wirbelströmung wurden eingangs die bisher in der Literatur unterschiedlich verwendeten Begriffe für die Reynoldszahlbereiche klar voneinander abgegrenzt. Experimentell wurden Kraftmessungen zur Bestimmung des zeitlich gemittelten und instationären Ablöseverhaltens genutzt, während Druckmessungen und Anstrichbilder mehr für die Ermittlung des räumlichen Ablöseverhaltens eingesetzt wurden. Für die Anwendung der verschiedenen Messverfahren wurden drei Zylindermodelle mit gleichem Zylinderdurchmesser im Hochdruckwindkanal Göttingen $(H D G)$ verwendet. Neben dem natürlichem, ungestörtem räumlich zeitlichen Ablöseverhalten wurde die Grenzschicht auch künstlich angeregt und in ihrer Stromabentwicklung beeinflusst. Dabei wurden stationäre wie instationäre, räumlich und zeitlich periodische Störungen in die Grenzschicht eingebracht.

Mit diesen experimentellen Untersuchungen wurde ein detailliertes Bild des laminar-turbulenten Überganges der ablösenden Zylindergrenzschicht erhalten (s. A.6 Re-Bereiche beim laminar-turbulenten Übergang der Zylindergrenzschicht). Die Transition erfolgt nicht durch ein gleichbleibendes, mit zunehmender Reynoldszahl stromauf wanderndes Instabilitätsszenario. Vielmehr werden mehrere stabile „Zwischenzustände“ eingenommen, die mit der Ablösung einer Querwirbelstruktur verbunden sind. Die räumliche Ablösung dieser Strukturen erfolgt quasi zweidimensional. Das instationäre Verhalten wird durch die periodische Ablösung der Querwirbel bestimmt. Da die Entstehung dieser Querwirbel auf der Wechselwirkung der instabilen freien abgelösten Scherschichten beruht, konnte ein neue Strouhalzahl $S r_{f}$ eingeführt werden. Sie wird anstelle des Durchmessers des Zylinders $D$ auf die sog. Rückkopplungslänge $L_{f}$ bezogen. Sie ist die zweifache Bogenlänge des Abstandes der Ablöseorte über dem hinteren Staupunkt. Für alle „Zwischenzustände“ mit instabilen freien Scherschichten im Ablösegebiet und Querwirbelablösung liefert diese Strouhalzahl einen konstanten Wert und wurde mit $S r_{f}=1,4 \pm 0,1$ angegeben. Wird ein solcher „Zwischenzustand“ der abgelösten Zylinderströmung instabil, ist dies mit Ausbildung spannweitig periodischer Längswirbel verknüpft. Dabei weisen diese vom Zylinder ablösenden Längswirbelstrukturen unterschiedliche spannweitige Längen auf. Sie wurden anhand spannweitiger Druckverteilungen und Anstrichbilder bestimmt. Im kritischen Reynoldszahlbereich sind die Längswirbel spannweitig kleinskalig und wechselwirken mit den ablösenden Grenzschichten. Mit dem Auftreten von Ablöseblasen wurden spannweitig periodische großskalige Längswirbelstrukturen beobachtet. Die Ausbildung von Längswirbelstrukturen bewirkt ein Instabilwerden der Querwirbel dominierten abgelösten Zylinderströmung. Mit Erreichen einer kritischen Reynoldszahl erfolgt dann der Übergang in den nächsten stabilen ,Zwischenzustand“.

Mit der einseitigen Einbringung von passiven wie aktiven Störungen in die Zylindergrenzschicht gelang es nicht nur bestimmte spannweitig periodische Wirbelstrukturen anzuregen oder zu stabilisieren, sondern auch widerstandsreduzierende Effekte zu erzielen.

Mit der aktiven Beeinflussung der Grenzschicht, stationär durch Absaugen und instationär durch akustische Anregung, wurde die „Transition A“ zu kleineren Reynoldszahlen verschoben. 
Dabei wurden die spannweitige Wellenlänge und zusätzlich bei der instationären Störung die Anregefrequenz bzw. Anregestrouhalzahl systematisch variiert. Im unterkritischen Reynoldszahlbereich konnten damit im Vergleich zur ungestörten, natürlich abgelösten Zylinderströmung Widerstandsreduktionen bis zu 50\% erreicht werden. Für den Vergleich der verschiedenen Anregungsverfahren wurde eine Reynoldszahl gemittelte Widerstandsreduktion $\overline{\Delta C_{W, r e l}}$ eingeführt. Insbesondere bei der akustischen Anregung zeigte sich, dass mit Erhöhung der Anregungsstrouhalzahl eine erhöhte mittlere Widerstandsreduktion bei konstanter Störamplitude zu verzeichnen war. Dieses Verhalten wurde auf die zunehmende Generierung von kleinskaligen Längswirbeln zurückgeführt, die sich aufgrund der großskalig spannweitig periodischen Anregung zu entsprechenden Längswirbelstrukturen vereinigten und bereits bei unterkritischen Reynoldszahlen zur „Transition A“ führten.

Bei der passiven Störung der Grenzschicht auf einer Seite des Zylinders wurden spannweitig äquidistant verteilte Wirbelerzeuger benutzt. Durch diese massive Störung wurde die Grenzschicht einseitig instabil und unterschiedliche asymmetrische Zustände bildeten sich im kritischen bis anfänglich transkritischen Reynoldszahlbereich aus. Mit Kraftmessungen und Anstrichbildern wurde gezeigt, dass auf der gestörten Seite des Zylinders eine entsprechende spannweitig periodische Längswirbelstruktur vom überkritischen bis anfänglich transkritischen Reynoldszahlbereich angeregt wurde. Damit erwiesen sich Wirbelerzeuger als ein mögliches effektives Instrument zur Widerstandsreduktion der turbulent abgelösten Zylinderströmung. 


\section{Literaturverzeichnis}

[1] Achenbach, E. (1979)

Strömung und konvektiver Wärmeübergang beim Kreiszylinder und bei der Kugel. KFAJül-1591, Kernforschungsanlage Jülich.

[2] Achenbach, E.; Heinecke, E. (1981)

On vortex shedding from smooth and rough cylinders in the range of Reynolds numbers $6^{\times} 10^{5}$ to $5^{\times} 10^{6}$. J. Fluid Mech. 109, 239-251.

[3] Allen, H.J. und Vincenti, W.G. (1944)

Wall interference in a two-dimensional flow wind tunnel, with consideration of the effect of compressibility. Nat. Adv. Comm. Aero., Wash., Rep. 782. (siehe auch Garner, H.C.; Rogers, E.W.E.; Acum, W.E.A.; Maskell, E.C.; AGARDograph 109, Subsonic wind tunnel wall corrections, October 1966.)

[4] Apelt, C.J. und West, G.S. (1975)

The effects of wake splitter plates on bluff body flow in the range of $10^{4}<\operatorname{Re}<5^{x} 10^{4}$. J. Fluid Mech. 71, 145-160.

[5] Bader, V. (1997)

Steuerung der Vorderkantenablösung an einem Tragflügelprofil mittels interner Schallanregung. Diplomarbeit. Georg-August-Universität Göttingen.

[6] Barkley, D.; Henderson R.D. (1996)

Three-dimensional Floquet stabilty analysis of the wake of a circular cylinder. J. Fluid Mech. 322, 215-241.

[7] Bearman, P.W. (1969)

On vortex shedding from a circular cylinder in the critical Reynolds number régime. J. Fluid Mech. 37, 577-586.

[8] Bendat, J.S.; Piersol, A.G. (1971)

Random data: Analysis and measurement procedures. Wiley-Interscience, New York.

[9] Bippes, H. (1990) aus Eckelmann, H. (1997) [24] S. 256.

[10] Blaquière, A. (1966)

Nonlinear system analysis. Academic Press, New York.

[11] Blevins, R.D. (1985)

The effect of sound on vortex shedding from cylinders. J. Fluid Mech. 161, 217-237.

[12] Bloor, M.S. (1964)

The transition to turbulence in the wake of a circular cylinder. J. Fluid Mech. 19, 290-304.

[13] Bloor, M.G.; Gerrard, J.H. (1966)

Measurement on turbulent vortices in a cylinder wake. Proc. Royal Society London A, 294, 319-342.

[14] Böttcher, J.; Wedemeyer, E. (1989)

The flow downstream of screens and its influence on the flow in the stagnation region of cylindrical bodies. J. Fluid Mech. 204, 501-522.

[15] Brede M.; Eckelmann, H.;Rockwell, D. (1996)

On secondary vortices in the cylinder wake. Phys. Fluids 8, 2117-2124. 
[16] Brede, M. (2000)

Private Kommunikation auf dem 12. DGLR-Fach-Symposium AGSTAB, 15.-17. Nov. 2000, Universität Stuttgart.

[17] Brede, M.; Leder, A. (2001)

On the structure of turbulence in the transitional cylinder wake. AGSTAB Stuttgart, Erscheint in: Notes on Numerical Fluid Mechanics (NNFM), Vieweg Verlag.

[18] Bushnell, D.M. (1992)

Longitudinal vortex control - techniques and applications. The 32nd Lanchester Memorial Lecture delivered at Royal Aeronautical Society, London, Tuesday 12 May 1992, Paper No. 1904; s. Aeronautical J. ,293 - 312.

[19] Dallmann, U. (1983)

Topological structures of three-dimensional flow separations. DLR, IB 221-82 A 07.

[20] Dallmann U., Schewe G. (1987)

On topological changes of separating flow structures at transition Reynolds numbers. AIAA 19th Fluid Dynamics, Plasma Dynamics and Laser Conference, June 8-10, 1987/ Honolulu, Hawaii. AIAA-87-1266.

[21] Dallmann U., Herberg Th., Gebing H., Su W.H., Zhang Q.H. (1995)

Flow field diagnostics: Topological flow changes and spatio-temporal flow structure. AIAA 33rd Aerospace Sciences, Meeting and Exhibit, January 9-12, 1995/Reno, NV; AIAA 95-0791.

[22] Darekar R.M., Sherwin S.J. (2000)

Flow past a bluff body with a wavy separation line. IUTAM Symposium on Bluff Body Wakes and Vortex-Induced Vibrations. BBVIV, 13-16 June 2000, Marseille, France. Erscheint in: J. Fluid Mech. (eingereicht 2000).

[23] Delany, N.K.; Sorrensen, N.E. (1953)

Low-speed drag of cylinders of various shapes. Nat. Adv. Comm. Aero., Washington, Technical Note 3038.

[24] Eckelmann, H. (1997)

Einführung in die Strömungsmeßtechnik. Teubner Studienbücher Mechanik. Leitfäden der angewandten Mathematik und Mechanik (LAMM), B.G. Teubner Stuttgart.

[25] Eisenlohr, H; Eckelmann, H. (1988)

Vortex splitting and its consequences in the vortex street wake of cylinders at low reynolds numbers. Phys. Fluids A 1, 189-1992.

[26] Feindt, E.G. (1956)

Untersuchungen über die Abhängigkeit des Umschlags laminar-turbulent von der Oberflächenrauhigkeit und der Druckverteilung. Dissertation Braunschweig. vgl. auch Jahrbuch 1956 der Schiffbautechnischen Gesellschaft 50, 180-203.

[27] Fey, U. (1997)

Eine neue Gesetzmäßigkeit für die Wirbelablösefrequenz des Kreiszylinders und Steuerung der Instabilitäten im Reyboldszahlbereich 160 bis 300. Dissertation, GeoergAugust-Universität Göttingen.

[28] Flachsbart, O. (1932)

Geschichte der experimentellen Hydro- und Aeromechanik, insbesondere der Widerstandsforschung. Handbuch der Experimentalphysik, Bd. 4, Akademische Verlagsgesellschaft, Leipzig, S. 1-57. 
[29] Försching, H.; Melzer, E.; Schewe, G. (1981)

Ein neuer Windkanal für gebäudeaerodynamische und -aeroelastische Untersuchungen bei Reynoldszahlen bis $10^{7}$. Sonderdruck aus ,konstruktiver ingenieurbau berichte“ 35 / 36,127-133. Vulkan-Verlag, Haus der Technik.

[30] Gad-el-Hak, M. and Bushnell, D.M. (1991)

Separation Control: Review. J. Fluids Eng. 113, 5-30.

[31] Gad-el-Hak, M. (1996)

Modern development in flow control. ASME Reprint NoAMR192, Appl. Mech. Review 49, 365-379.

[32] Haken, H. (1992)

Eine Einführung in die Synergetik. Springer Verlag, New York, Berlin, 1992.

[33] Hein S., Thefilis, V., Dallmann U. (1998)

Unsteadiness and three-dimensionality of steady two-dimensional laminar separation bubbles as result of linear instability mechanisms. DLR-IB 223-98 A39.

[34] Higuchi, H.; Kim, H.J.; Farell, C. (1989)

On flow separation and reattachment around a circular cylinder at critical Reynolds numbers. J. Fluid Mech. 200, 149-171.

[35] Hoerner, W. (1958)

Fluid-dynamic drag. Washington D.C.

[36] Hsiao, F.B.; Shyu, J.Y. (1991)

Influence of internal acoustic excitation on the vortex-shedding of a circular cylinder. $\mathrm{J}$. Fluids Struct. 5, 427-442.

[37] Humphreys, J.S. (1960)

On a circular cylinder in a steady wind. J. Fluid Mech. 9, 603-612.

[38] Huerre, P.; Monkewitz, P.A. (1990)

Local and global instabilities in spatially developing flows. Ann. Rev. Fluid Mech. 22, 473-537.

[39] Koch, W. (1985)

Local instability characteristics and frequency determination of self-excited wake-flows. J. Sound Vibration 99, 53-83.

[40] Kraemer, K. (1961)

Über die Wirkung von Stolperdrähten auf den Grenzschichtumschlag. Z. Flugwissenschaft 9, 20-27.

[41] König, M. (1993)

Experimentelle Untersuchung des dreidimensionalen Nachlaufs zylindrischer Körper bei kleinen Reynoldszahlen. Mitt. aus dem Max-Planck-Inst. für Strömungsforschung. Hrsg. E.-A. Müller, Nr. 111.

[42] König, M; Noack, B.R.; Eckelmann, H. (1993)

Discrte shedding modes in the von Kármán vortex street. Phys. Fluids A 5, 1846-1848.

[43] Leder, A. (1992)

Abgelöste Strömungen: physikalische Grundlagen. Braunschweig, Widesbaden, Vieweg Verlag.

[44] Lin, J.C.; Selby, G.V.; Howard, F.G. (1991)

Exploratory study of vortex-generating devices for turbulent flow separation control. AIAA-91-0042. 
[45] Lin, J.-C.; Vorobieff, P.; Rockwell, D. (1994)

Three-dimensional patterns of streamwise vorticity in hte turbulent near-wake of a cylinder. J. Fluids Struct. 9, 231-235.

[46] Mechel, F.P. (1989)

Schalabsorber. Band 1. Äussere Schallfelder, Wechselwirkungen. S. Hirzel Verlag, Stuttgart.

[47] Meneghini J.R., Bearman P.W. (1992)

Numerical simulation of control of bluff body flow using a discrete vortex method incorporating viscous diffusion. IUTAM Symposium Göttingen/Germany 1992. Bluff-Body Wakes, Dynamics and Instabilities. Eds.: H. Eckelmann, J.M.R. Graham, P. Huerre, P.A. Monkevitz. Springer Verlag, 257-262.

[48] Messing, R.; Kloker, M.; Wagner S. (1999)

Direct numerical simulation of suction through discrete holes in a three-dimensional boundary layer. NNFM, New results in numerical and experimental fluid dynamics II. 11th AGSTAB/DGLR Symposium Berlin, Germany, Nov. 10-12, 1998, Vieweg Verlag, 323-330.

[49] Meyer, E. und Guicking, D. (1974)

Schwingungslehre. Friedr. Vieweg\&Sohn, Braunschweig.

[50] Mittal, R.; Balachandar, S. (1994)

Effect of three-dimensionality on the lift and drag of circular and elliptic cylinders. TAM Report No. 774, University of Illinois at Urbana-Champaign.

[51] Mittal, R., Balachandar S. (1995)

Autogeneration of three-dimensional vortical structures in the near wake of a circular cylinder. TAM Report No. 812, Universitiy of Illinois at Urbana-Champaign.

[52] Morkovin, M.V. (1964)

Flow around circular cylinder - a kaleidoscope of challenging fluid phenomena. Proc. ASME Symposium in Fully Separated Flows, Philadelphia, 102-118.

[53] Morkovin, M.V. (1984)

Bypass transition to turbulence and and research desiderata. Transitions in Turbines Symposium, NASA CP-2386, Washingtopn D.C.

[54] Morsbach, M. (1967)

Über die Bedingungen für eine Wirbelstraßenerzeugung hinter Kresizylindern. Dissertation, RWTH Aachen.

[55] Niemann, H.-J.; Hölscher, N. (1990)

A review of recent experiments on the flow past circular cylinders. J. Wind Eng. Ind. Aerod. 33, 197-209.

[56] Norberg, C. (1994)

An experimental investigation of the flow around a circular cylinder: influence of aspect ratio. J. Fluid Mech. 258, 287-316.

[57] Oertel, H.; Delfs, J. (1996)

Strömungsmechanische Instabilitäten. Berlin, Springer Verlag.

[58] Owen, J.C.; Bearman, P.W.; Szweczyk A.A. (2000)

The influence of streamwise vorticity on von Kármán vortex shedding. IUTAM Symposium on Bluff Body Wakes and Vortex-Induced Vibrations. BBVIV, 13-16 June 2000, Marseille, France. 
[59] Prandtl, L. (1904)

Über Flüssigkeitsbewegungen bei sehr kleiner Reibung. Verhandlungen des III. Internationalen Mathematikerkongresses, Heidelberg, 484-491.

[60] Prandtl, L. (1914)

Der Luftwiderstand von Kugeln. Nachrichten der Gesellschaft der Wissenschaften zu Göttingen, Mathematisch-physikalische Klasse, 177-190.

[61] Prandtl, L. (1921)

Neuere Einsichten in die Gesetze des Luftwiderstandes. Festschrift der Kaiser-WilhelmGesellschaft zur Förderung der Wissenschaften zum 10jährigen Jubiläum, Berlin, Springer Verlag, 178-184.

[62] Prandtl, L. (1922)

Bemerkungen über die Entstehung der Turbulenz. Zeitschrift für angewandte Mathematik und Mechanik (ZAMM) 1, 431-436. Physikalische Zeitschrift 23, 19-25.

[63] Prandtl, L. (1926)

Bericht über neuere Turbulenzforschung. Hydraulische Probleme. Berlin, VDI-Verlag, 114.

[64] Raguse, A. (1998)

Experimentelle Untersuchungen zur aktiven dynamischen Beeinflussung des laminar-turbulenten Umschlags in einer Plattengrenzschicht. DLR-FB 98-29.

[65] Relf, E.F.; Simmons, L.F.G. (1924)

The frequency of eddies generated by the motion of circular cylinders through a fluid. Aero. Res. Counc., Lond. Rep. and Mem., no. 917.

[66] Reynolds, O. (1883)

Phil. Trans. Roy. Soc. London 1883. (s. Schlichting 1960 [81], S. 13)

[67] Richter, A.; Naudascher, E. (1976)

Flutuating forces on a rigid circular cylinder in confined flow. J. Fluid Mech. 78, 561-576.

[68] Rist, U.; Maucher, U., Wagner, S. (1996)

Direct numerical simulation of some fundamental problems related to transition in laminar separation bubbles. In Proc. Computational Fluid Dynamics Conf. ECCOMAS '96, 319-325.

[69] Rockwell, D. (1983)

Oscillations of impinging shear layers. AIAA J. 21, 645-664.

[70] Roshko, A. (1954)

On the drag and shedding frequency of two-dimensional bluff-bodies. NACA TN 3169.

[71] Roshko, A. (1961)

Experiments on the flow past a circular cylinder at very high Reynolds number. J. Fluid Mech. 10, 345-356.

[72] Roshko, A. (1993)

Perspectives on bluff body aerodynamics. J. Wind Eng. Ind. Aerod. 49, 79-100.

[73] Saric, W.S.; Carillo, R.B.; Reibert, M.S. (1998)

Leading edge roughness as a transition control mechanism. AIAA-98-02781.

[74] Schewe, G. (1982)

A multicomponent balance consisting of piezoelectric force transducers for a high-pressure windtunnel. Conf. Proc. Sensor \& Systems '82, vol. 2, Pasadena (USA), May 18-20, 1982; (Deutsche Übersetzung in Techn. Messen 49, 447-458.) 
[75] Schewe, G. (1983)

On the force fluctuations acting on a circular cylinder in crossflow from subcritical up to transcritical Reynolds numbers. J. Fluid Mech. 133, 265-285.

[76] Schewe, G. (1985)

Experimental observation of the 'Golden Section' in flow around a circular cylinder. Phys. Letter 109A, 47-51.

[77] Schewe G. (1986)

Sensitivity of transition phenomena to small perturbations in flow round a circular cylinder. J. Fluid Mech. 172, 33-46.

[78] Schewe, G. (2000)

Reynolds-number effects in flow around more-or-less bluff bodies. Bluff Body Aerodynamics and Applications BBAA IV, Universität Bochum. Erscheint in: J. Wind Eng. Ind. Aerod.

[79] Schewe, G. (2000)

Private Kommunikation. Institut für Aeroelastik, DLR, Göttingen, 2000.

[80] Schiller, L. und Linke, W. (1933)

Druck- und Reibungswiderstand des Zylinders bei Reynoldsschen Zahlen 5000 bis 40000. Zeitschrift Flugtechnik und Motorluft 24, 193-198.

[81] Schlichting, H. (1960)

Boundary-layer theory. Series in Mechanical Engineering. McGraw-Hill Book Company, 1960.

[82] Schubauer, G.B. and Skramstad, H.K. (1947)

Laminar boundary-layer oscillations and stability of laminar flow. J. Aeron. Sciences 14, 69-78.

[83] Siller, H.A.; Fernholz, H.H. (1998)

Manipulation of trailing edge separation on an aerofoil using periodic cross-flow. IUTAM Symposium on „Mechanics of passive and active flow control“. Eds.: G.E.A. Meier and P.R. Viswanath, Proceedings of the IUTAM Symposium, Göttingen, Germany, 7-11 September 1998, Kluwer Academic Publisher.

[84] Steinhoff, Chr. (1999)

Experimente zu Mach- und Reynoldszahleffekten bei der Umströmung eines Kreiszylinders unter kryogenen Bedingungen. Dissertation, Georg-August-Universität Göttingen und DLR-FB 99-02.

[85] Strouhal, V. (1878)

Über eine besondere Art der Tonanregung. Ann. Physik und Chemie. Neue Folge 5, 216251.

[86] Strykowski, P.J.; Sreenivasan, K.R. (1990)

On the formation and suppression of vortex ,shedding“ at low Reynolds numbers. J. Fluid Mech. 218, 71-107.

[87] Sucker, D. und Brauer, H. (1975)

Fluiddynamik bei querangeströmten Zylindern. Wärme- und Stoffübertragung 8, 149158.

[88] Tamura T. , Ohta I., Kuwahara K. (1990)

On the reliability of two-dimensional simulation for unsteady flows around a cylindertype structure. J. Wind Eng. Ind. Aerod. 35, 275-298. 
[89] Taneda, S. (1978)

Visual observation of the flow past a sphere at Reynolds numbers between $10^{4}$ and $10^{6}$. J. Fluid Mech. 85, 187-192.

[90] Theofilis V., Hein S, Dallmann U. (2000)

On the origins of unsteadiness and three-dimensionality in a laminar separation bubble. Erscheint in: Phil. Trans. Roy. Soc. London A (eingereicht 2000).

[91] Tichy, J.; Gautschi, G. (1980)

Piezo-elektrische Meßtechnik. Springer Verlag, Berlin.

[92] Unal, M.F.; Rockwell, D. (1988)

On vortex formation from a cylinder. Part 1. The imitial instability. J. Fluid Mech. 190, 491-512.

[93] von Kármán, Th. (1911)

Über den Mechanismus des Widerstandes, den ein bewegter Körper in einer Flüssigkeit erfährt. Nachrichten der Kaiserlichen Gesellschaft der Wissenschaften zu Göttingen. Mathematisch-physikalische Klasse, 9 Seiten.

[94] Wei T., Smith C.R. (1986)

Secondary vortices in the wake of circular cylinders. J. Fluid Mech. 169, 513-533.

[95] Wieselsberger, C; Betz, A. (1923)

Versuche über den Luftwiderstand gerundeter und kantiger Körper. Hrsg.: Prandtl, L.; Ergebnisse der Aerodynamischen Versuchsanstalt zu Göttingen. II. Lieferung. München und Berlin Verlag R. Oldenbourg, 22-35.

[96] Williamson, C.H.K. (1996)

Three-dimensional wake transition. J. Fluid Mech. 328, 345-407.

[97] Wu J., Sheridan J., Hourigan K., Soria J. (1996)

Shear-layer vortices and longitudinal vortices in the near wake of a circular cylinder. Exp. Thermal Fluid Sciences 12, 169-174.

[98] Wu, X.H.; Wu, J.Z.; Wu, J.M. (1991)

Guiding principles for vortex flow control. AIAA-91-0617.

[99] Zdravkovich, M.M. (1990)

Conceptual overview of laminar and turbulent flows past smooth and rough circular cylinders. J. Wind Eng. Ind. Aerod. 33, 53-62.

[100] Zdravkovich, M.M. (1997)

Flow around circular cylinder. Volume 1, Oxford University Press.

[101] Zhang, H.Q.; Noack, B.R.; Eckelmann, H. (1994)

Numerical computation of the 3-D cylinder wake. Max-Planck-Insitut für Strömungsforschung, Göttingen, Bericht 3/1994.

[102] Zhang, H.Q.; Fey, U.; Noack, B.R.; König, M.; Eckelmann, H. (1995)

On the the transition of the cylinder wake. Phys. Fluids 7, 779. 


\section{A. Anhang}

\section{A.1 $R e$-Bereiche beim laminar-turbulenten Übergang der Zylindergrenzschicht}

\begin{tabular}{|c|c|c|c|c|c|}
\hline & $\begin{array}{l}\text { unter- } \\
\text { kritisch }\end{array}$ & kritisch & $\begin{array}{c}\text { über- } \\
\text { kritisch }\end{array}$ & $\begin{array}{c}\text { oberer } \\
\text { Übergang }\end{array}$ & $\begin{array}{l}\text { trans- } \\
\text { kritisch }\end{array}$ \\
\hline$R e_{D} /\left[10^{6}\right]$ & $0,04 \ldots 0,20$ & $0,20 \ldots 0,38$ & $0,38 \ldots 1,0$ & $1,0 \ldots 2,2$ & $2,2 \ldots$ \\
\hline$C_{W}$ & $1,25 \ldots 1,10$ & $1,10 \ldots 0,22$ & 0,22 & $0,22 \ldots 0,4$ & $0,4 \ldots$ \\
\hline$\left|C_{A}\right|$ & 0,0 & $\begin{array}{l}0,0 \\
1,4 \ldots 0,9 \\
0,0\end{array}$ & 0 & $0(?)$ & 0 \\
\hline$S r$ & 0,20 & $\begin{array}{lll}0,20 & \ldots & 0,16 \\
0,33 & \ldots & 0,30 \\
0,48 & \ldots & 0,52\end{array}$ & 0,52 & $\begin{array}{l}0,10 \\
\text { (breitbandig) }\end{array}$ & $0,20 \ldots$ \\
\hline$c_{A, e f f}$ & $0,40 \ldots 0,20$ & $0,20 \ldots 0,02$ & 0,02 & $0,02 \ldots 0,10$ & $0,05 \ldots$ \\
\hline $\begin{array}{l}\text { Zustand der } \\
\text { Grenzschicht } \\
\text { unmittelbar } \\
\text { stromauf der } \\
\text { Ablösung }\end{array}$ & laminar & laminar & laminar & laminar & turbulent \\
\hline
\end{tabular}

Tabelle A1 Charakteristische Werte von Widerstands-, Auftriebsbeiwert, Strouhalzahl sowie Effektivwert der Auftriebsschwankungen in den jeweiligen Reynoldszahlbereichen beim laminar-turbulenten Übergang der ablösenden Zylindergrenzschicht 


\section{A.2 Einteilung der Reynoldszahlbereiche nach verschiedenen Autoren}

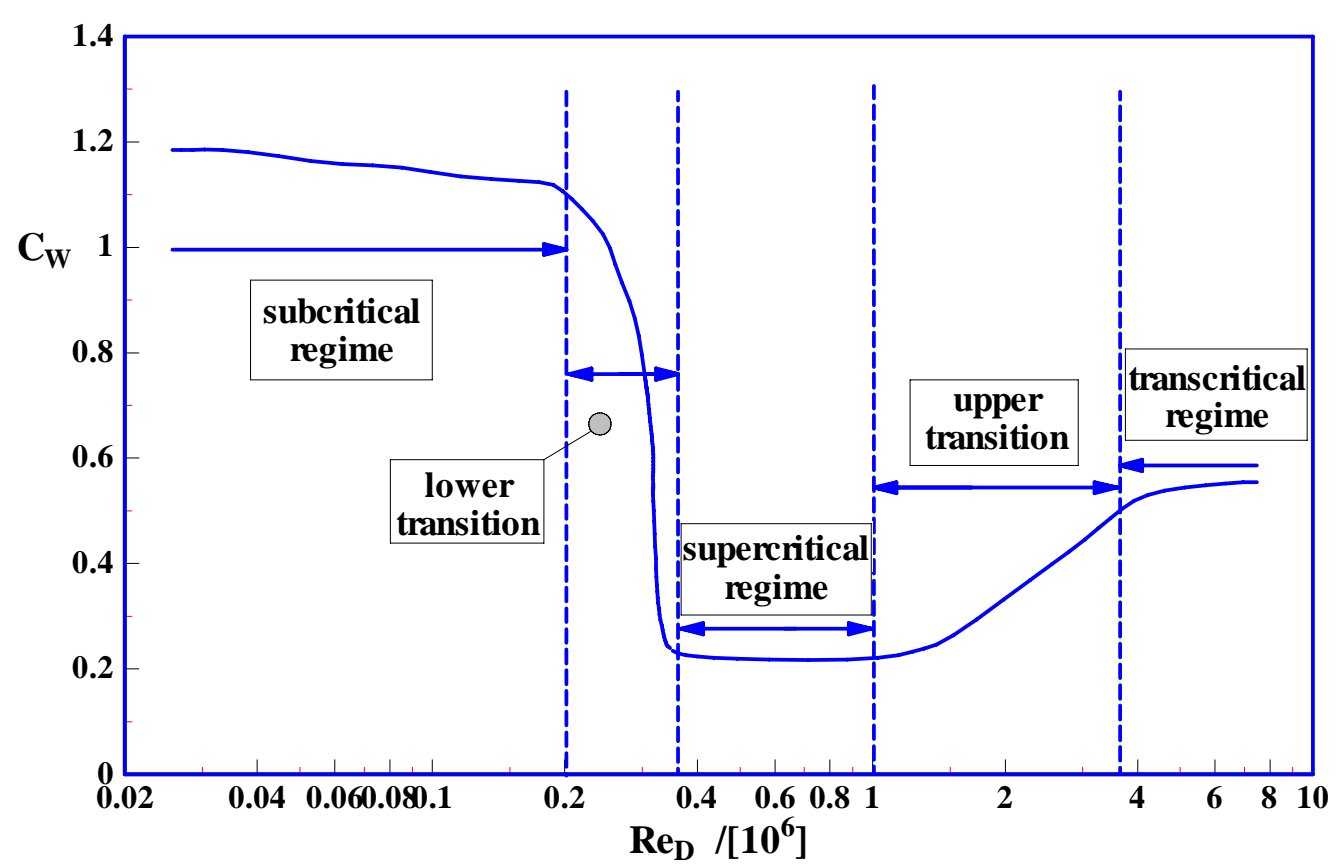

Bild A.1.1 Einteilung der Reynoldszahlbereiche beim laminar-turbulenten Übergang der Zylindergrenzschicht nach Roshko (1961) [71]

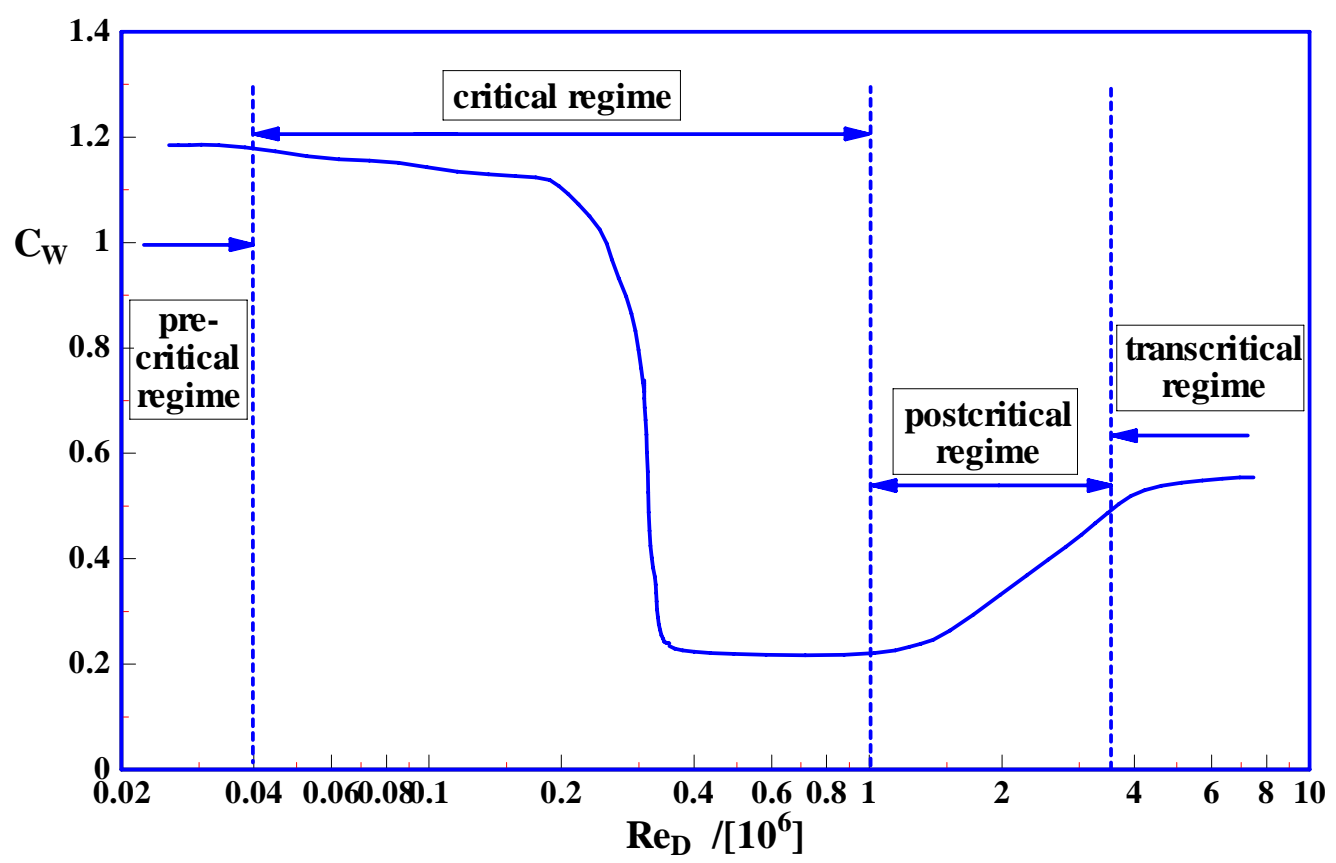

Bild A1.2 Einteilung der Reynoldszahlbereiche beim laminar-turbulenten Übergang der Zylindergrenzschicht nach Morkovin (1964) [52] 


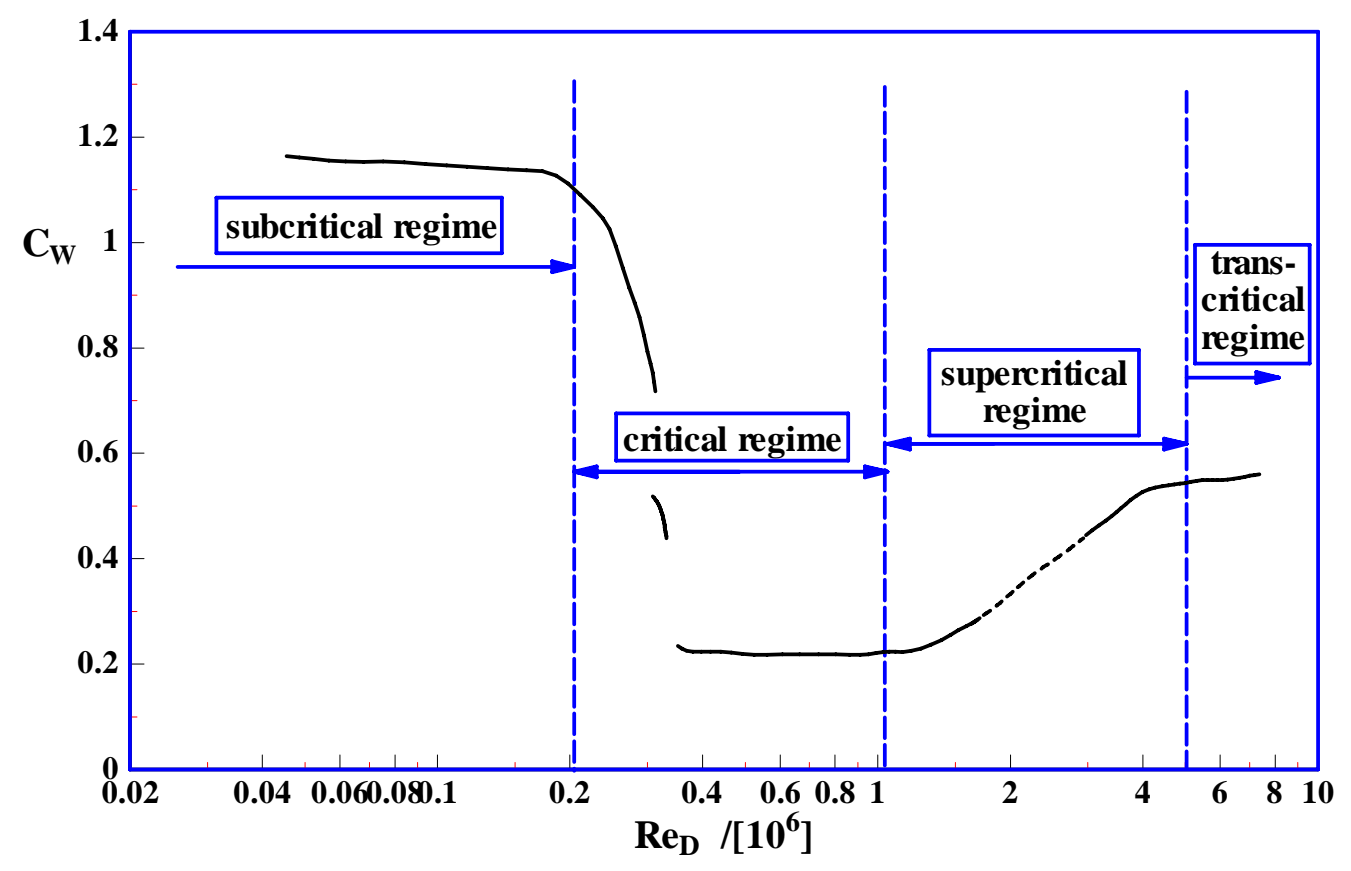

Bild A1.3 Einteilung der Reynoldszahlbereich beim laminar-turbulenten Übergang der Zylindergrenzschicht nach Niemann und Hölscher (1990) [55]

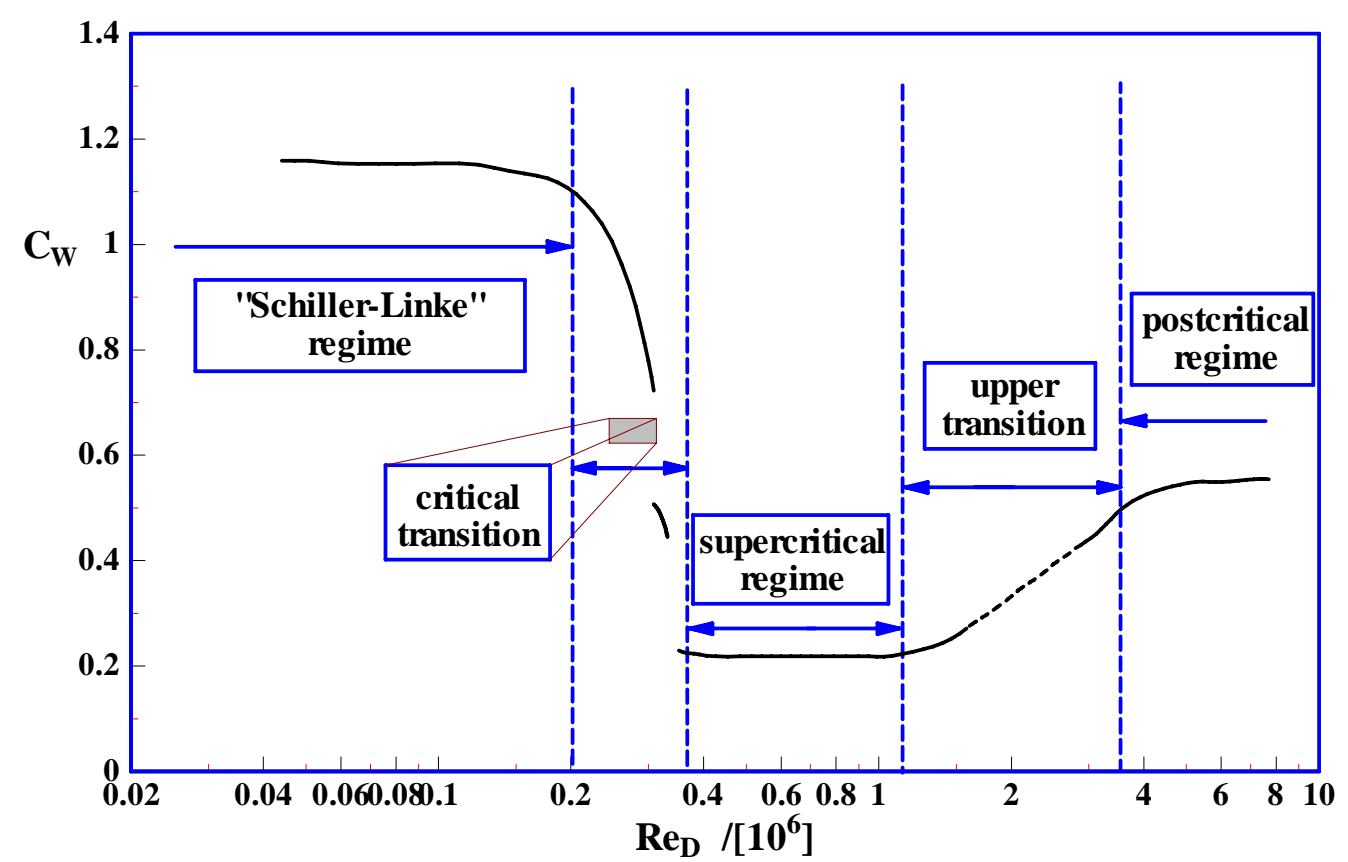

Bild A1.4 Einteilung der Reynoldszahlbereich beim laminar-turbulenten Übergang der Zylindergrenzschicht nach Roshko (1993) [70] 


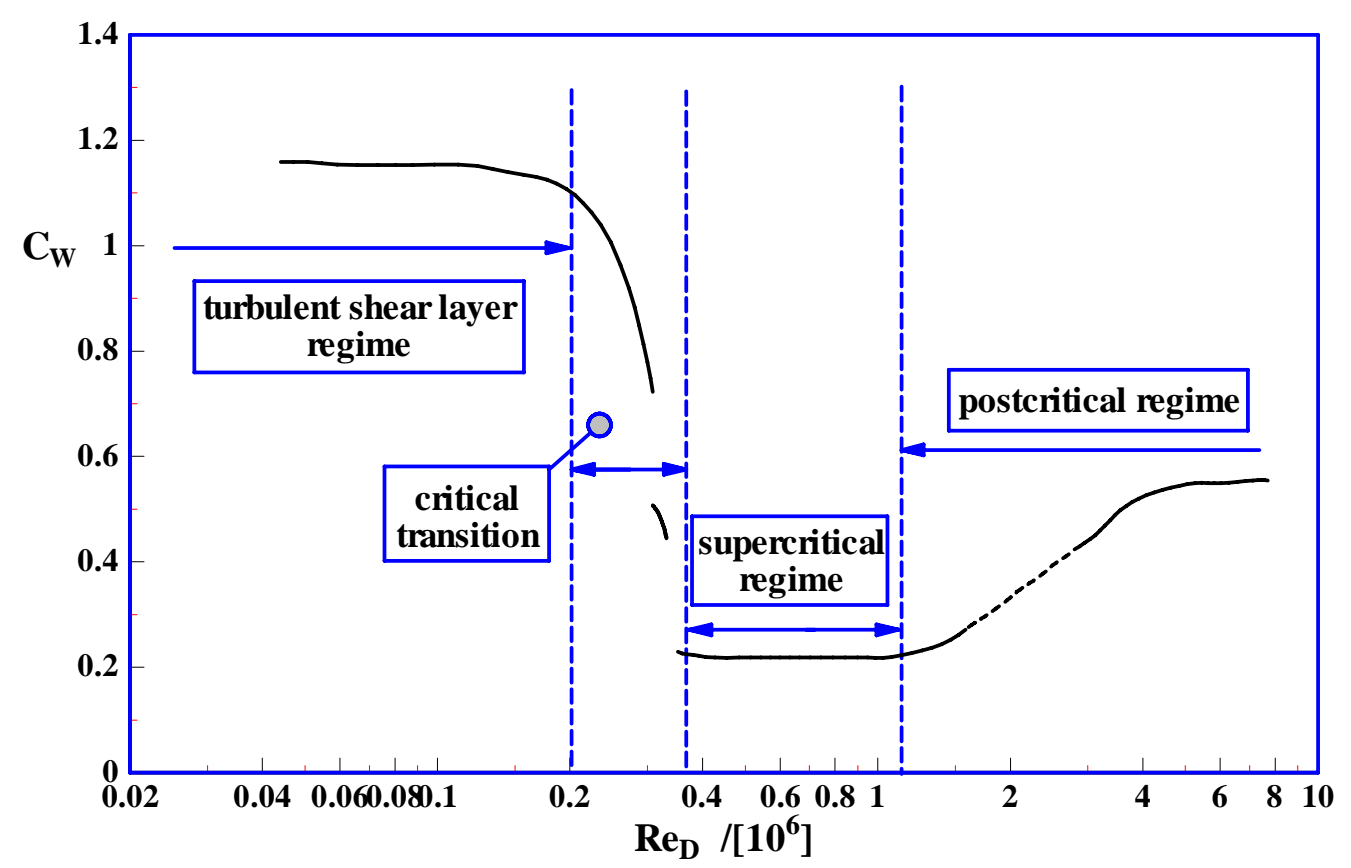

Bild A1.5 Einteilung der Reynoldszahlbereich beim laminar-turbulenten Übergang der Zylindergrenzschicht nach Williamson (1996) [96]

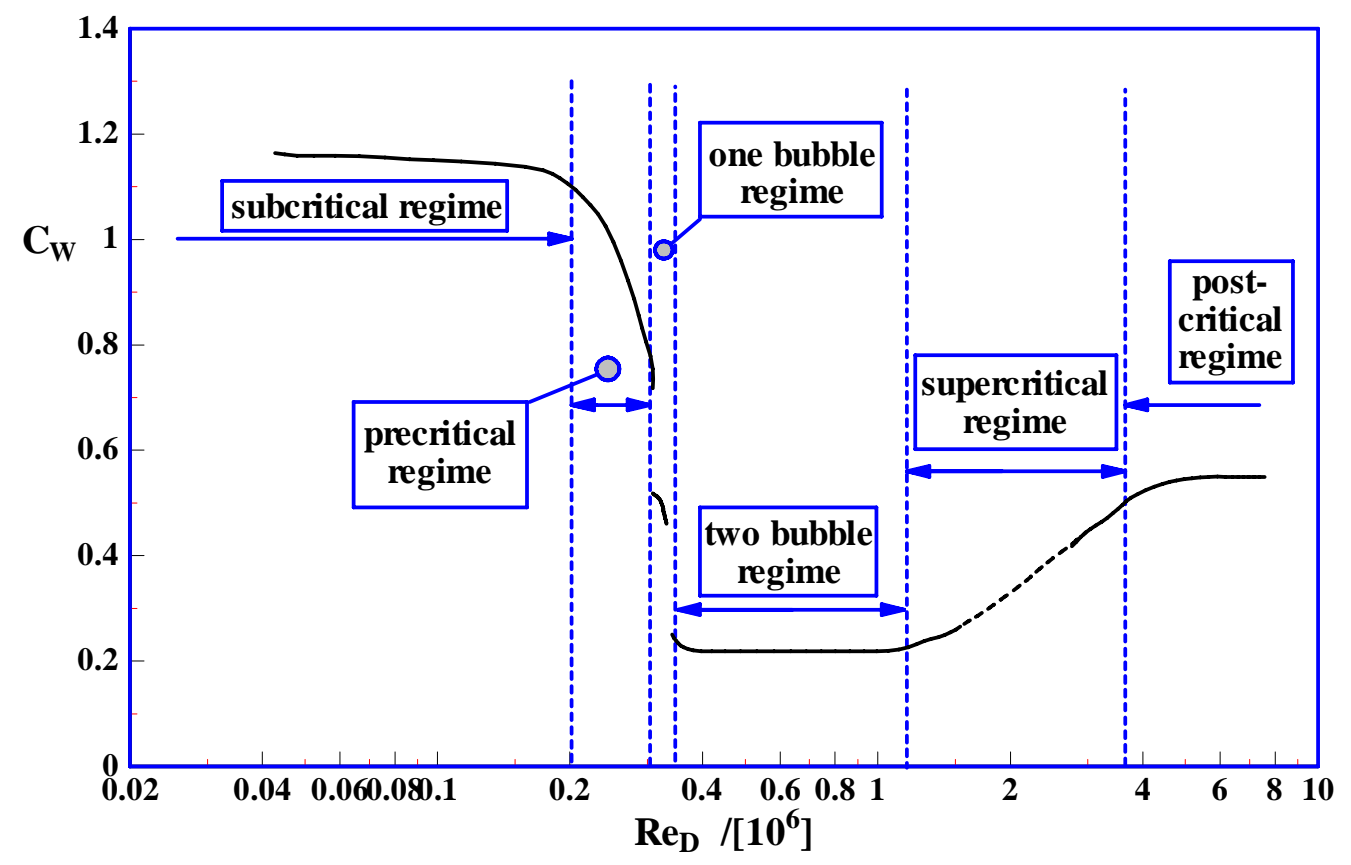

Bild A1.6 Einteilung der Reynoldszahlbereich beim laminar-turbulenten Übergang der Zylindergrenzschicht nach Zdravkovich (1997) [100] 


\section{A.3 Anstrichbilder der natürlich abgelösten Zylinderströmung}

\begin{tabular}{|c|c|c|c|c|c|}
\hline & $\begin{array}{c}R e_{D} \\
/\left[10^{6}\right]\end{array}$ & $C_{W}$ & $S r$ & $\begin{array}{c}\varphi_{S, \text { oben }} \\
/[\mathrm{Grad}]\end{array}$ & $\begin{array}{c}\varphi_{S, \text { unten }} \\
/[\mathrm{Grad}]\end{array}$ \\
\hline \hline Bild A2.1 & 0,12 & 1,15 & 0,21 & 76 & 76 \\
\hline Bild A2.2 & 0,15 & 1,10 & 0,20 & 76 & 76 \\
\hline Bild A2.3 & 0,17 & 1,10 & 0,20 & 76 & 76,90 \\
\hline Bild A2.4 & 0,19 & 1,08 & 0,20 & 76,91 & 76,91 \\
\hline Bild A2.5 & 0,20 & 1,12 & 0,22 & 76,91 & 76,91 \\
\hline Bild A2.6 & 0,32 & 0,70 & 0,18 & 82,98 & 82,98 \\
\hline Bild A2.7 & 0,34 & 0,52 & 0,31 & 76,90 & $90 . .95$ \\
& & & & & $110 . .135$ \\
\hline Bild A2.8 & 0,41 & 0,32 & $k . A$. & 90 & 90 \\
\hline Bild A2.9 & 0,60 & 0,41 & $k . A$. & $95 . .135$ & $95 . .135$ \\
\hline Bild A2.10 & 0,80 & 0,46 & $k . A$. & $95 . .130$ & $95 . .130$ \\
\hline Bild A2.11 & 1,00 & 0,60 & $(0,28)$ & $95 / 105$ & $95 / 105$ \\
\hline Bild A2.12 & 1,60 & 0,74 & $(0,32)$ & 92 & 92 \\
\hline Bild A2.13 & 3,00 & 0,90 & $k . A$. & 88 & 88 \\
\hline & & & & & $100 . .135$ \\
\hline & & & & & \\
\hline
\end{tabular}

Tabelle A2 Übersicht über angefertigte Anstrichbilder bei verschiedenen Reynoldszahlen des laminar-turbulenten Überganges. Bei der Anfertigung der Anstrichbilder wurde zumeist nur der Bereich um den vorderen Staupunkt eingestrichen. 


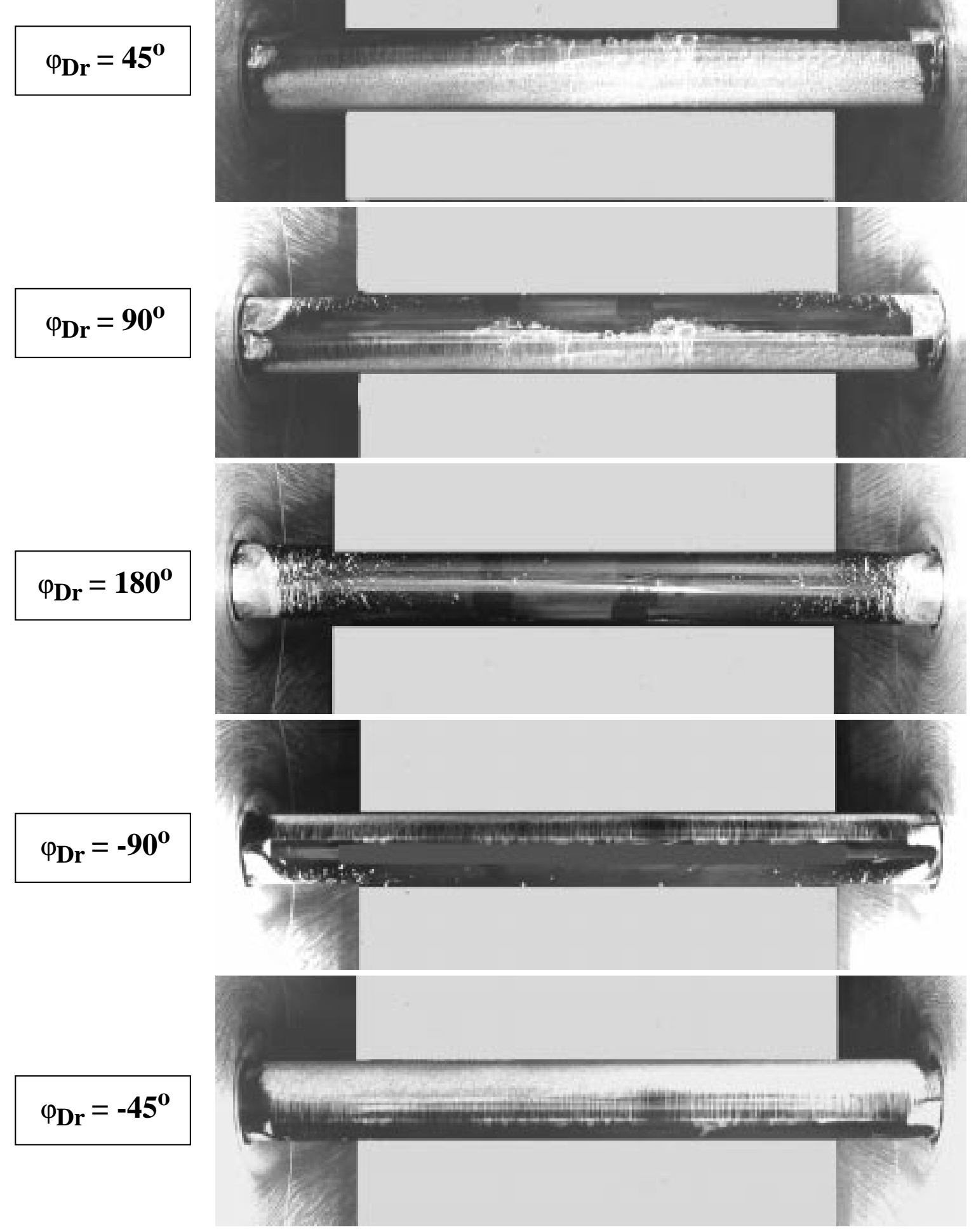

Bild A2.1 Ölanstrichbild bei gemessener Reynoldszahl von $R e_{D}=0,12^{\times} 10^{6}$ mit $C_{W}=1,15$ 


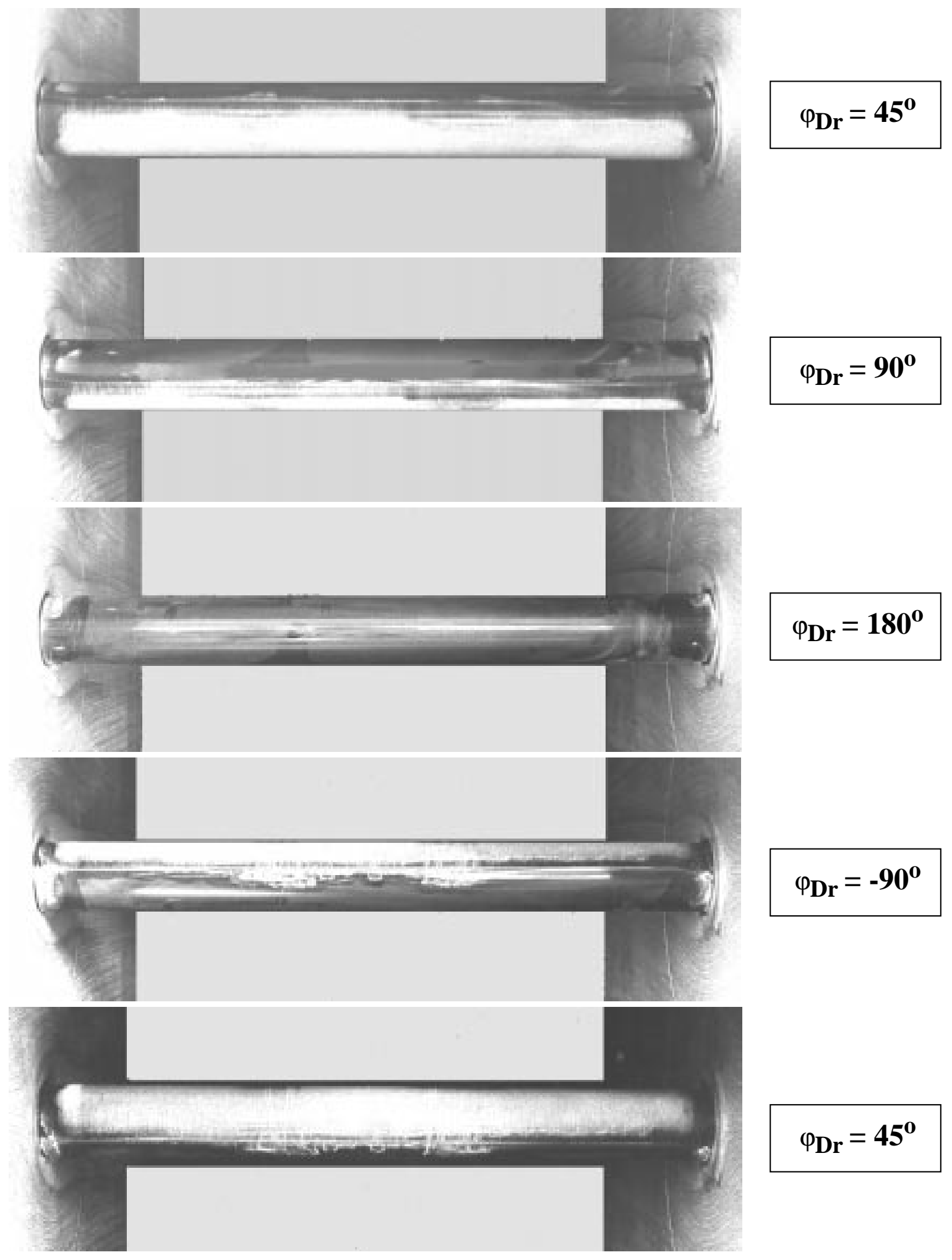

Bild A2.2 Ölanstrichbild bei gemessener Reynoldszahl von $R e_{D}=0,15^{\times} 10^{6}$ mit $C_{W}=1,10$ 

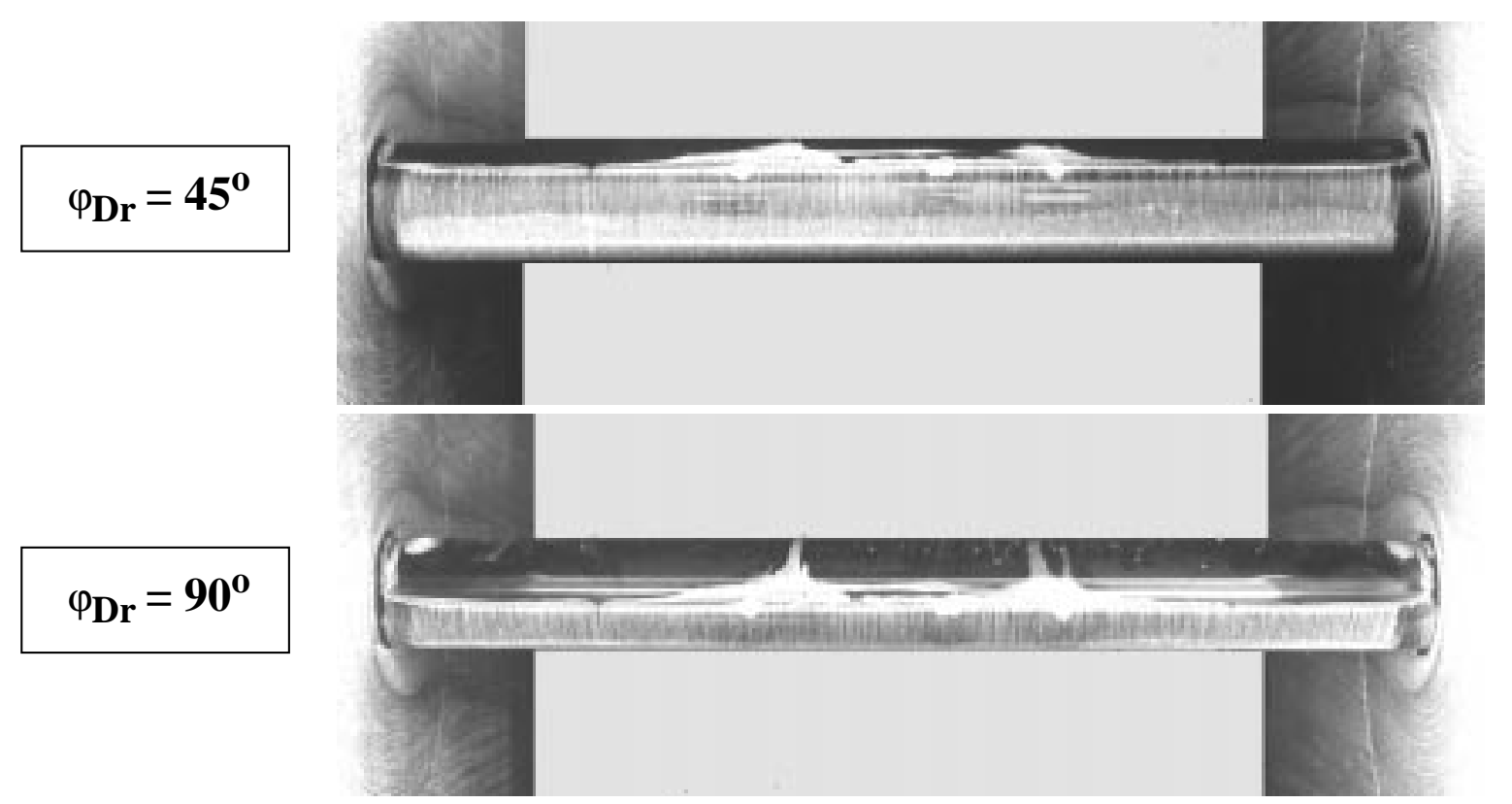

$$
\varphi_{\mathrm{Dr}}=\mathbf{1 8 0}^{\mathbf{0}}
$$

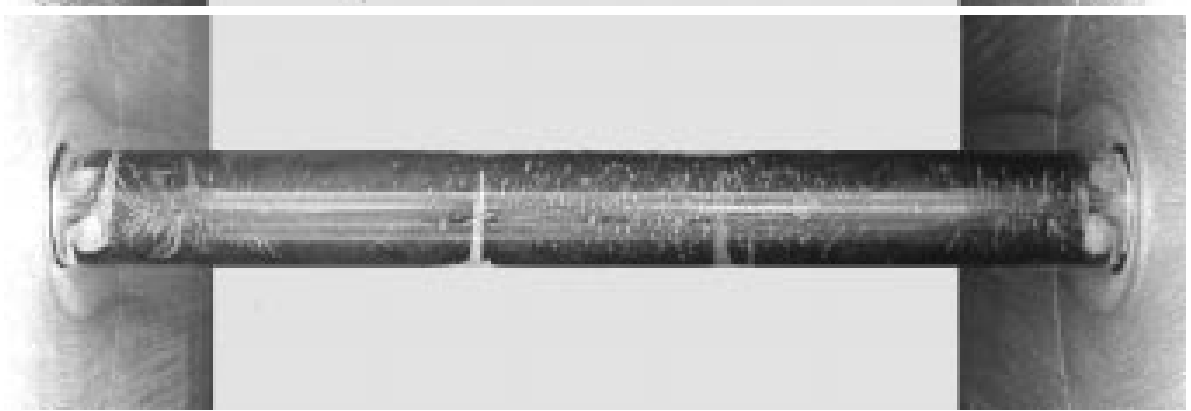

$$
\varphi_{\text {Dr }}=-\mathbf{- 9 0}^{\circ}
$$

$$
\varphi_{\mathrm{Dr}}=-\mathbf{4 5}
$$
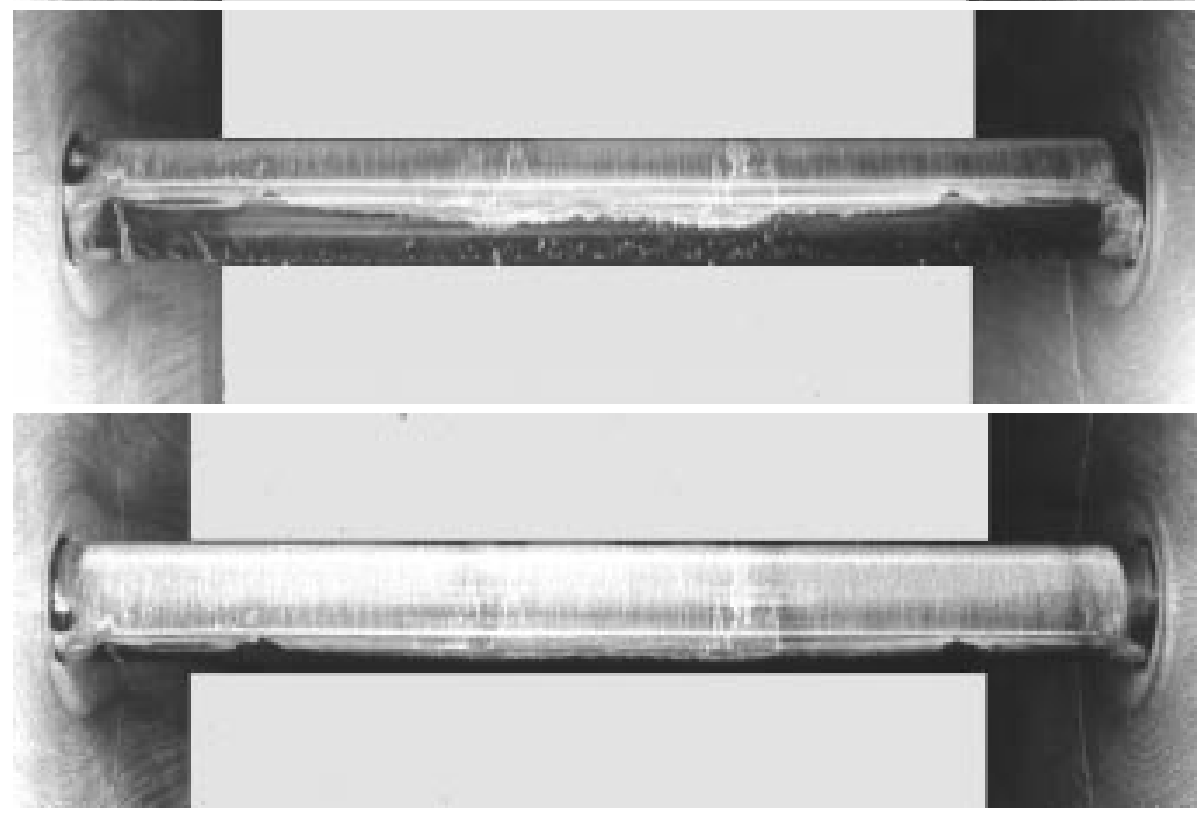

Bild A2.3 Ölanstrichbild bei gemessener Reynoldszahl von $R e_{D}=0,17 \times 10^{6}$ mit $C_{W}=1,10$ 


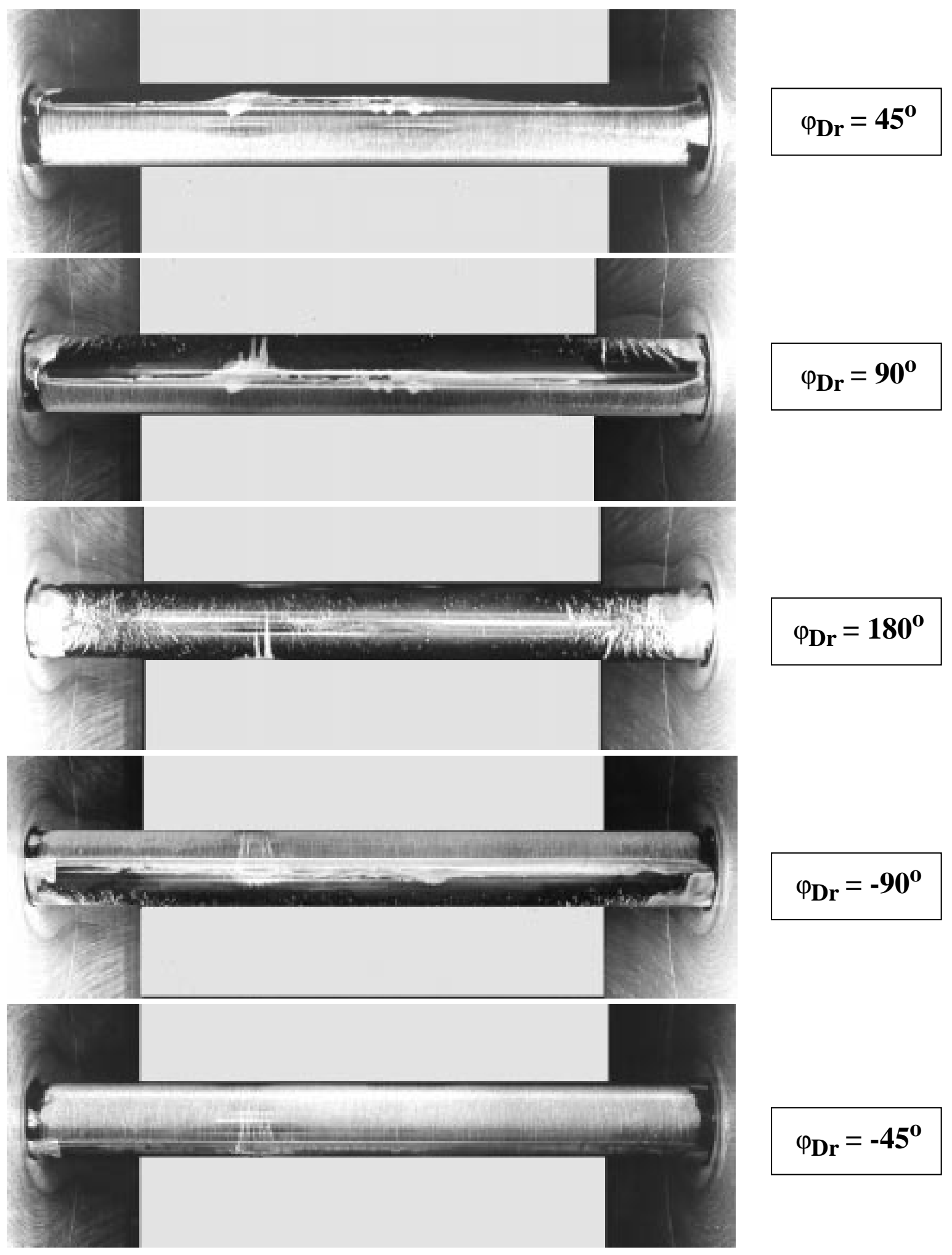

Bild A2.4 Ölanstrichbild bei gemessener Reynoldszahl von $R e_{D}=0,19^{\times} 10^{6}$ mit $C_{W}=1,08$ 


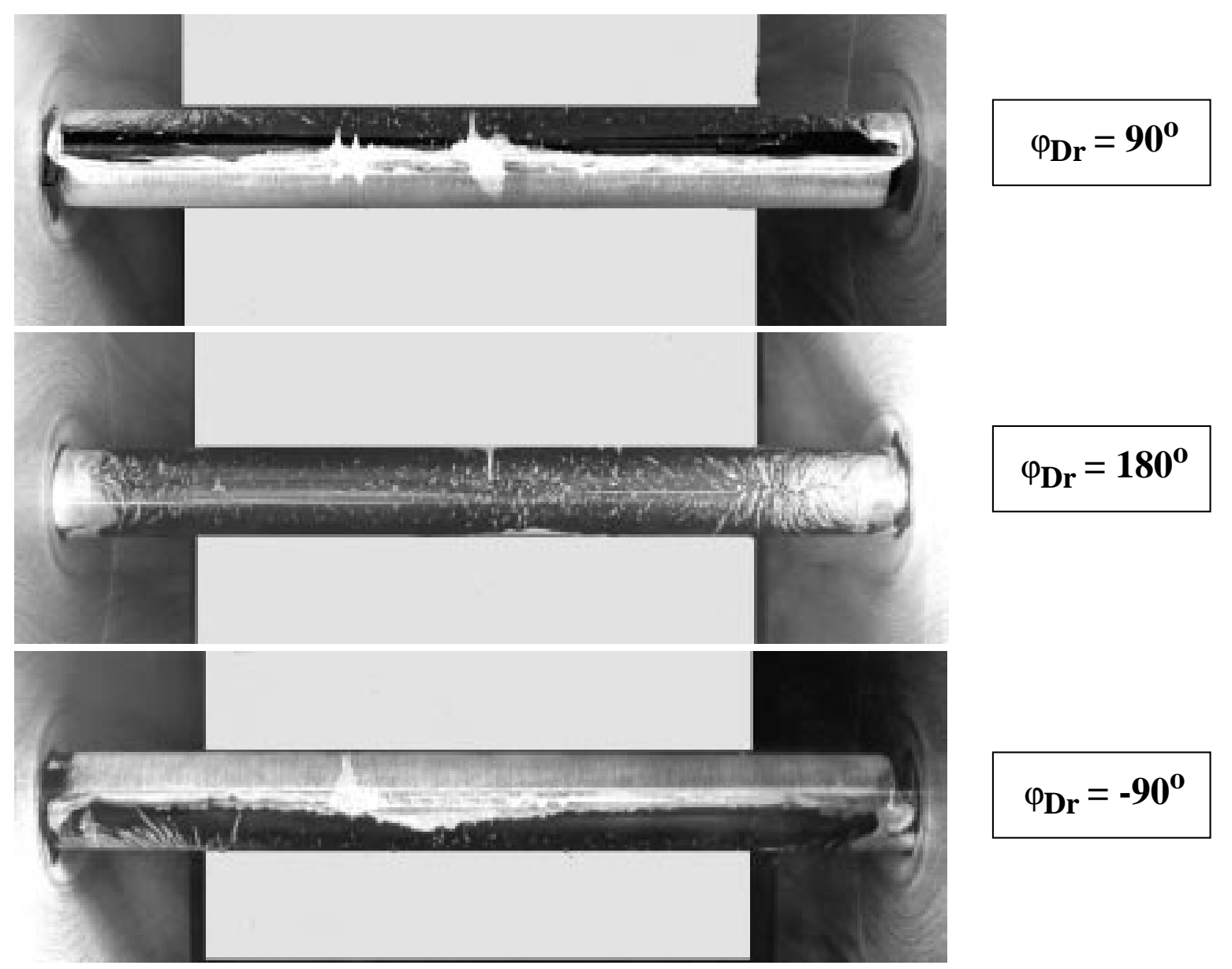

Bild A2.5 Ölanstrichbild bei gemessener Reynoldszahl von $R e_{D}=0,20^{\times} 10^{6}$ mit $C_{W}=1,12$ 


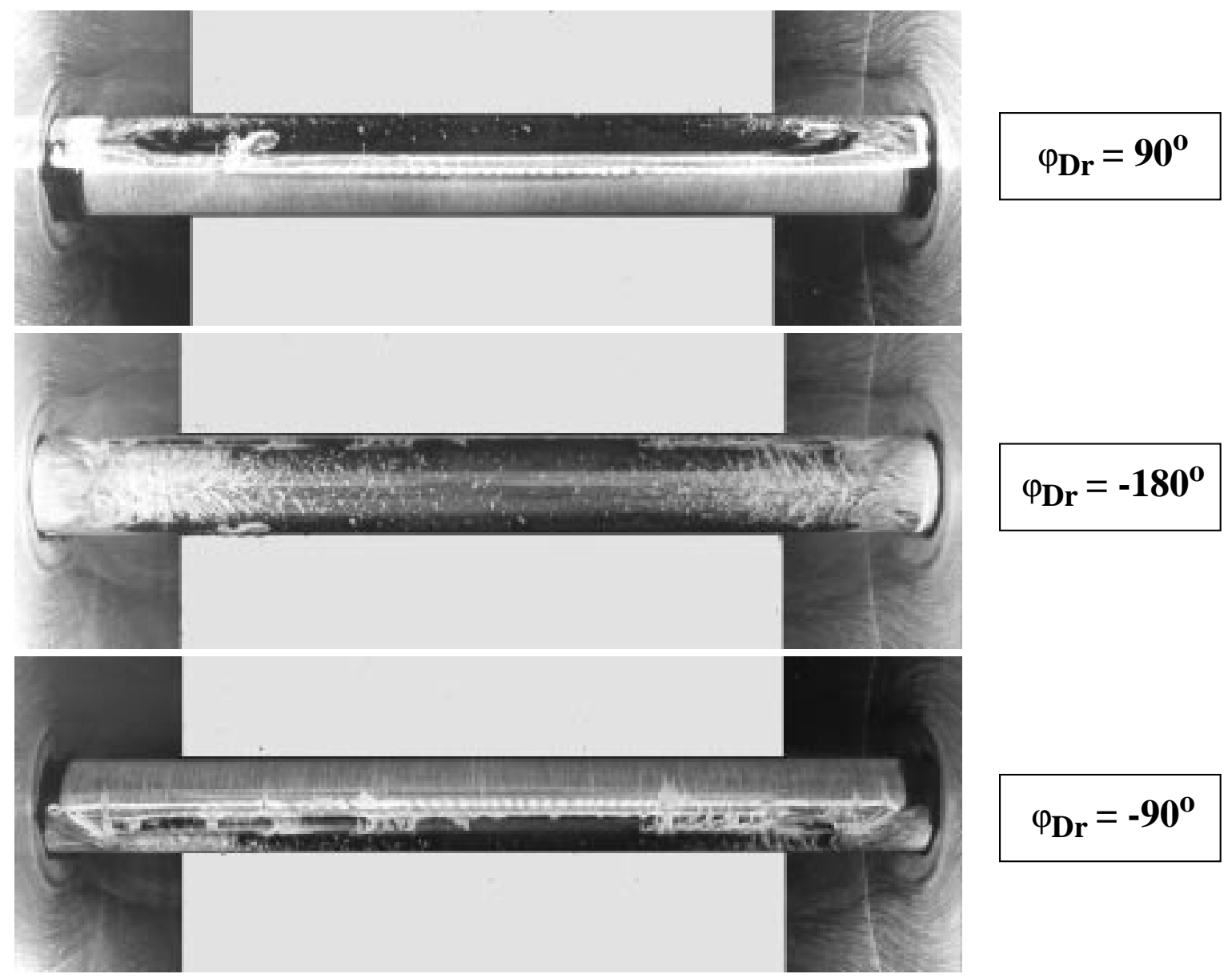

Bild A2.6 Ölanstrichbild bei gemessener Reynoldszahl von $R e_{D}=0,32^{\times} 10^{6}$ mit $C_{W}=0,70$ 


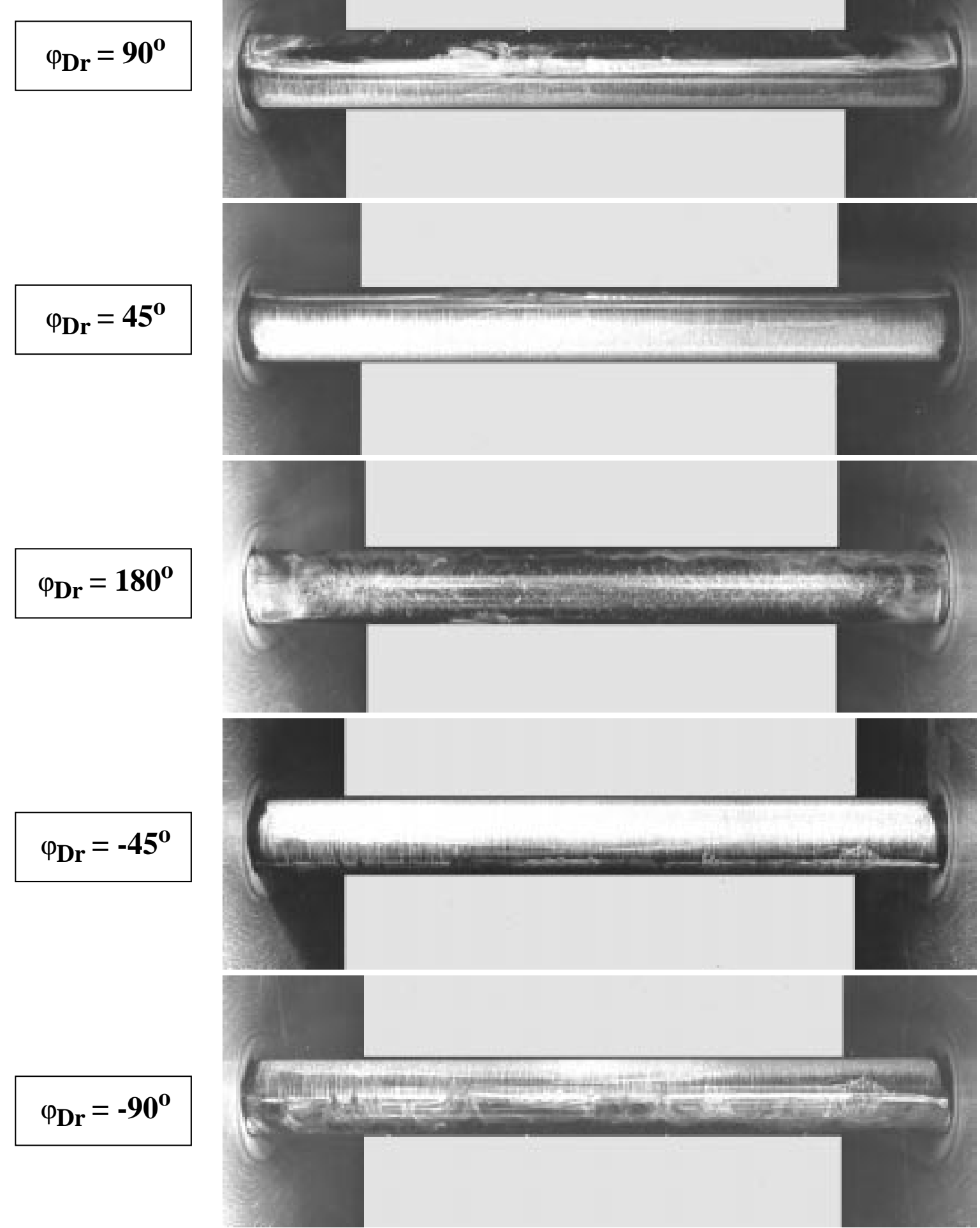

Bild A2.7 Ölanstrichbild bei gemessener Reynoldszahl von $R e_{D}=0,34 \times 10^{6}$ mit $C_{W}=0,52$ 


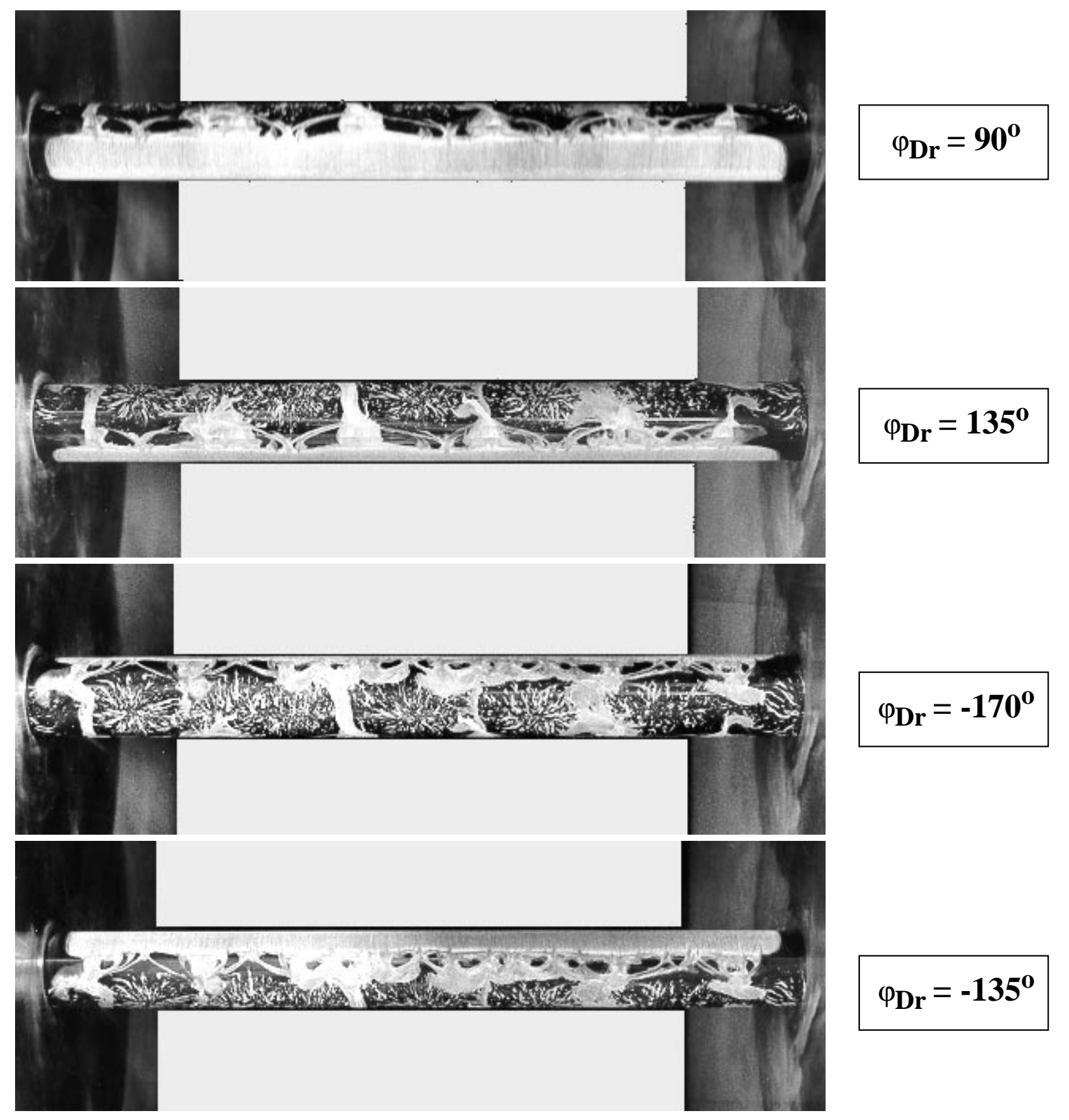

Bild A2.8 Ölanstrichbild bei gemessener Reynoldszahl von $R e_{D}=0,41^{\times 10^{6}}$ mit $C_{W}=0,32$ 


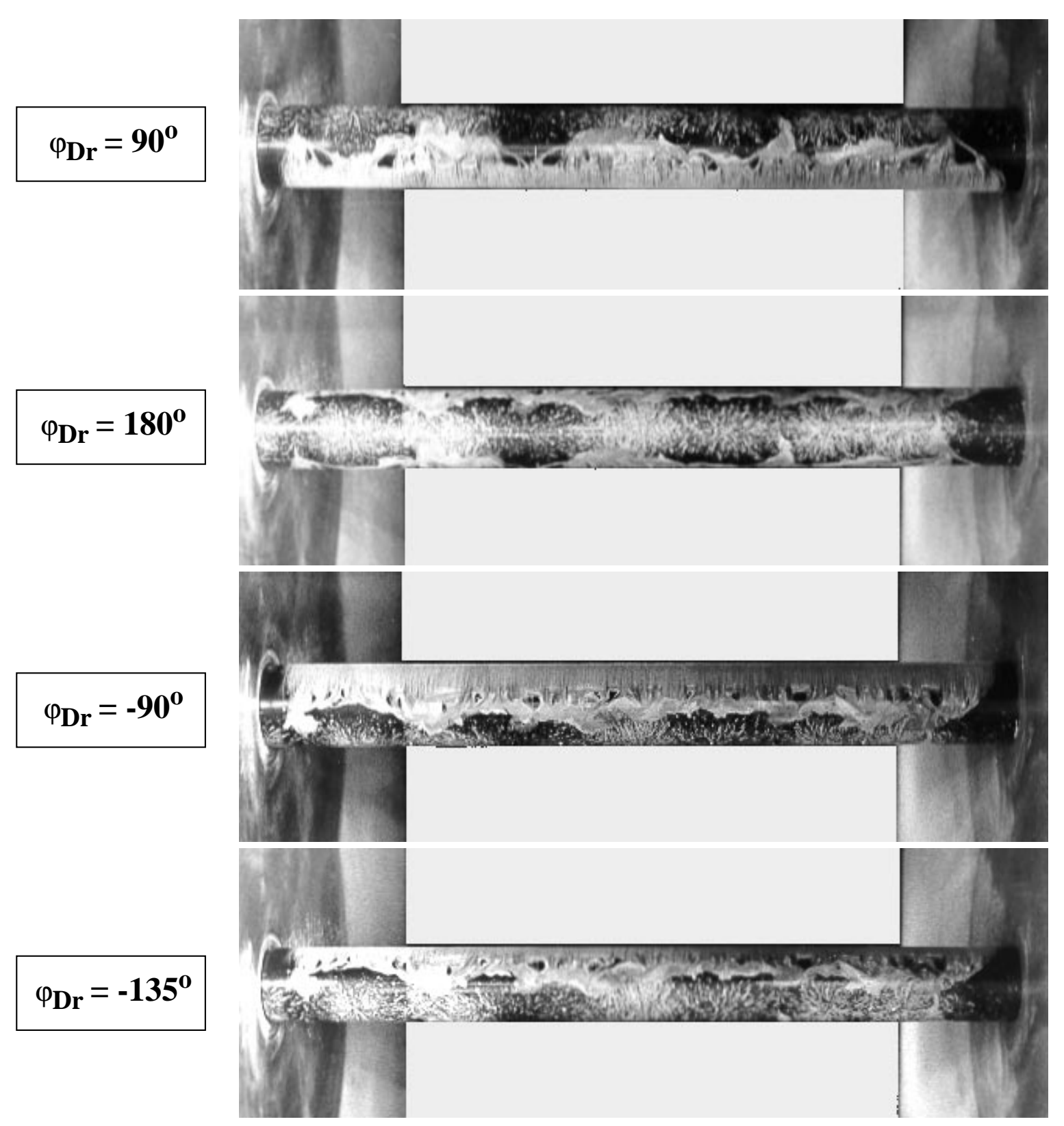

Bild A2.9 Ölanstrichbild bei gemessener Reynoldszahl von $R e_{D}=0,60^{\times} 10^{6}$ mit $C_{W}=0,41$ 


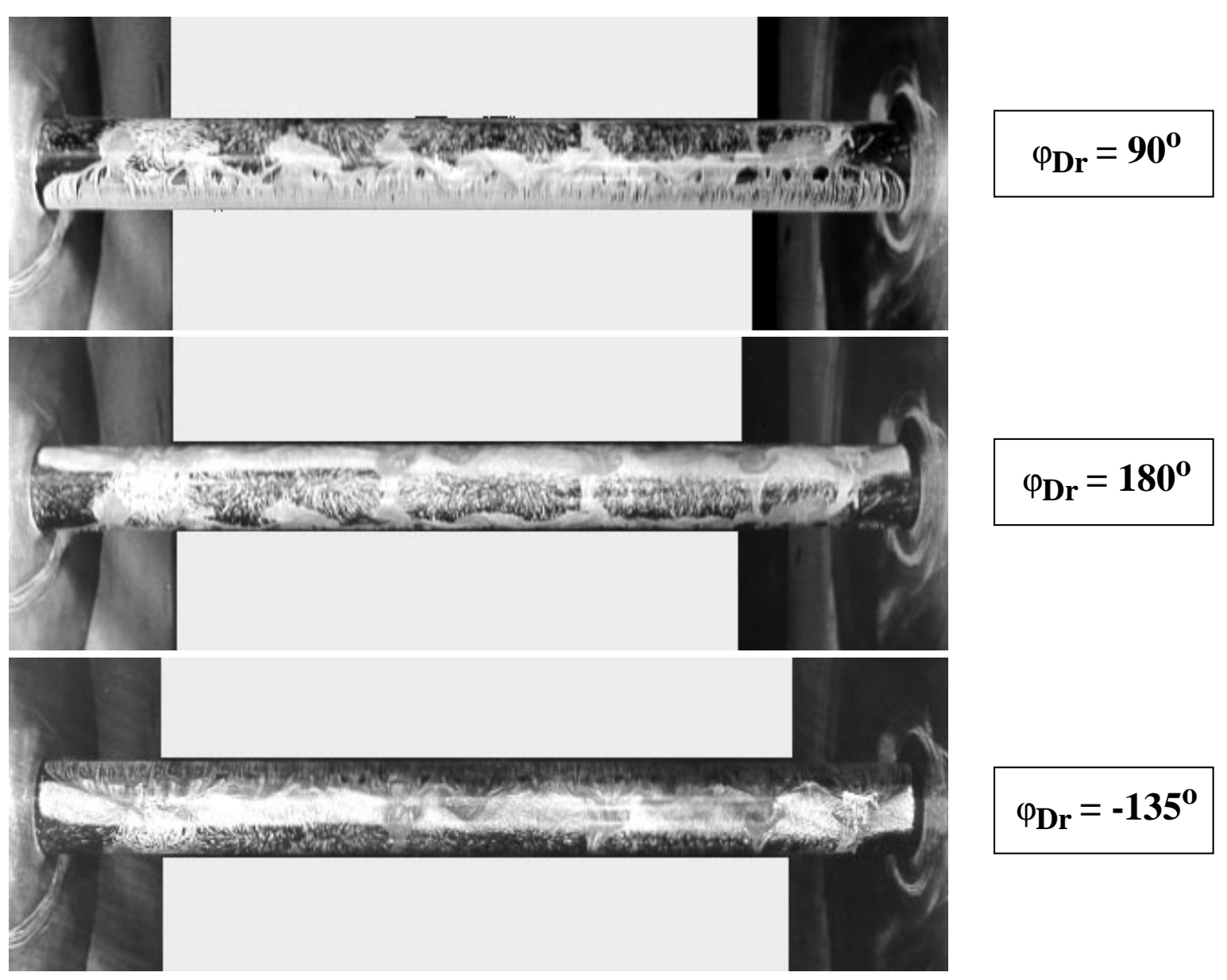

Bild A2.10 Ölanstricbild bei gemessener Reynoldszahl von $R e_{D}=0,80^{\times 10^{6}}$ mit $C_{W}=0,46$ 


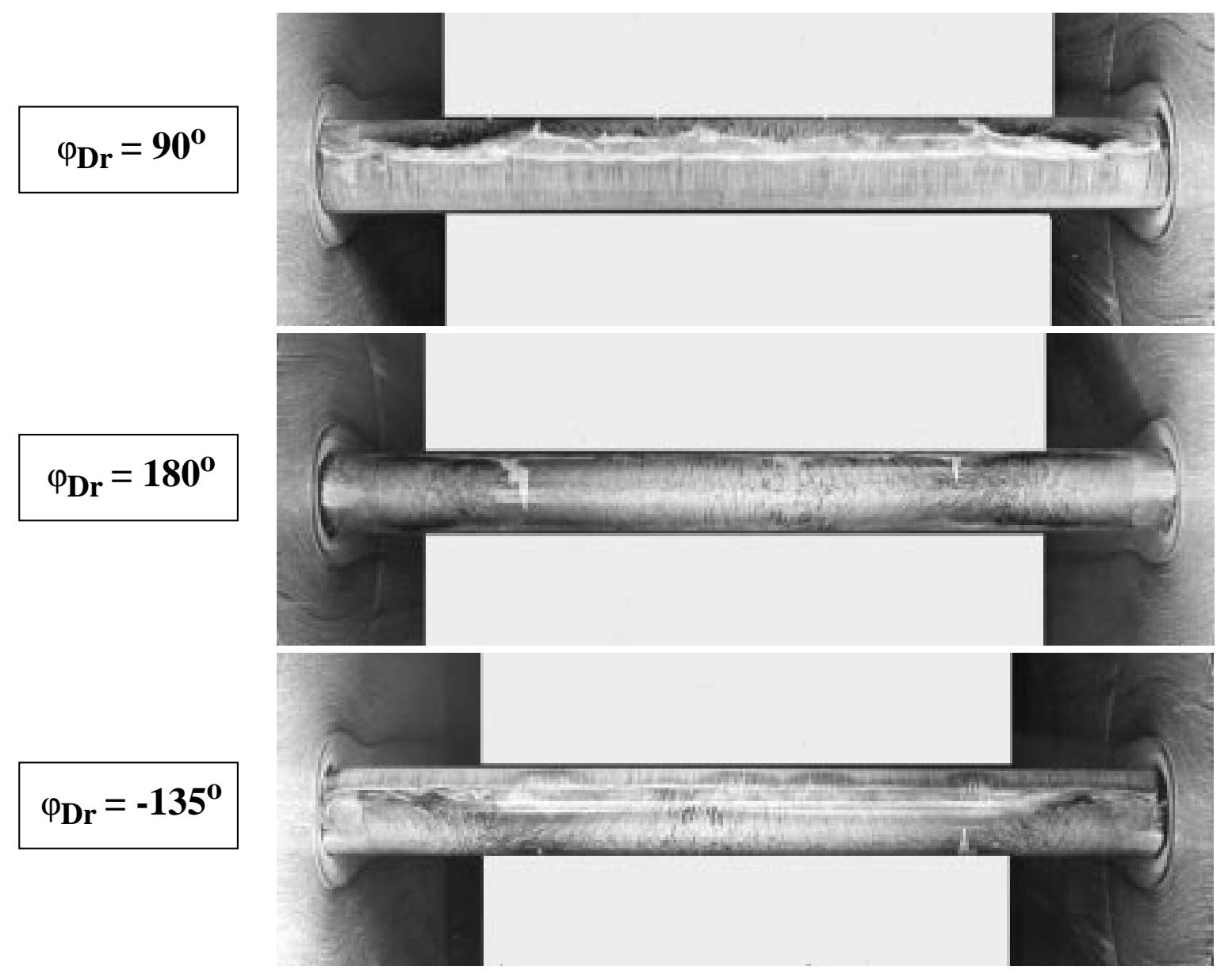

Bild A2.11 Ölanstrichbild bei gemessener Reynoldszahl von $R e_{D}=1,00 \times 10^{6}$ mit $C_{W}=0,60$ 


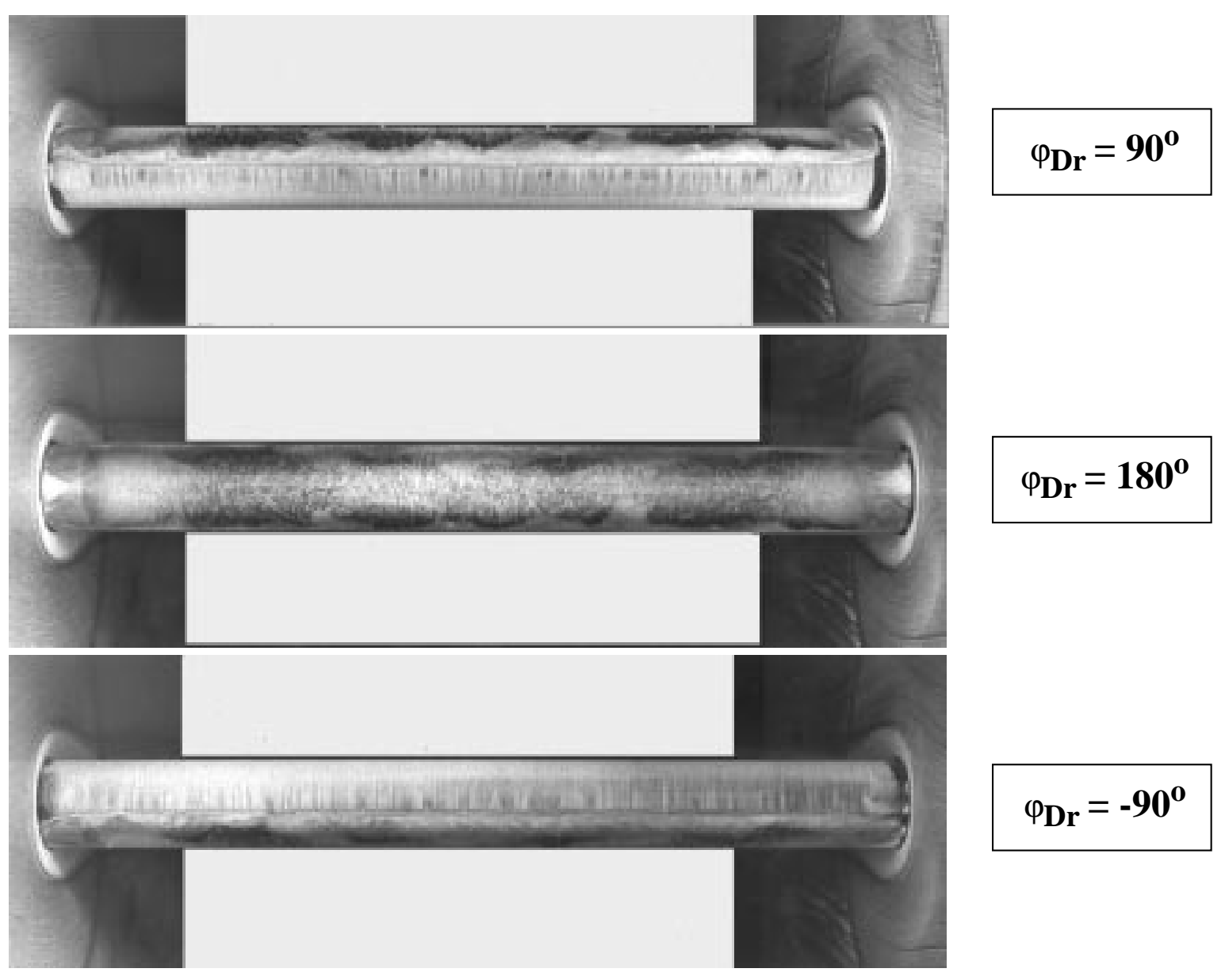

Bild A2.12 Ölanstrichbild bei gemessener Reynoldszahl von $R e_{D}=1,60 \times 10^{6}$ mit $C_{W}=0,74$ 


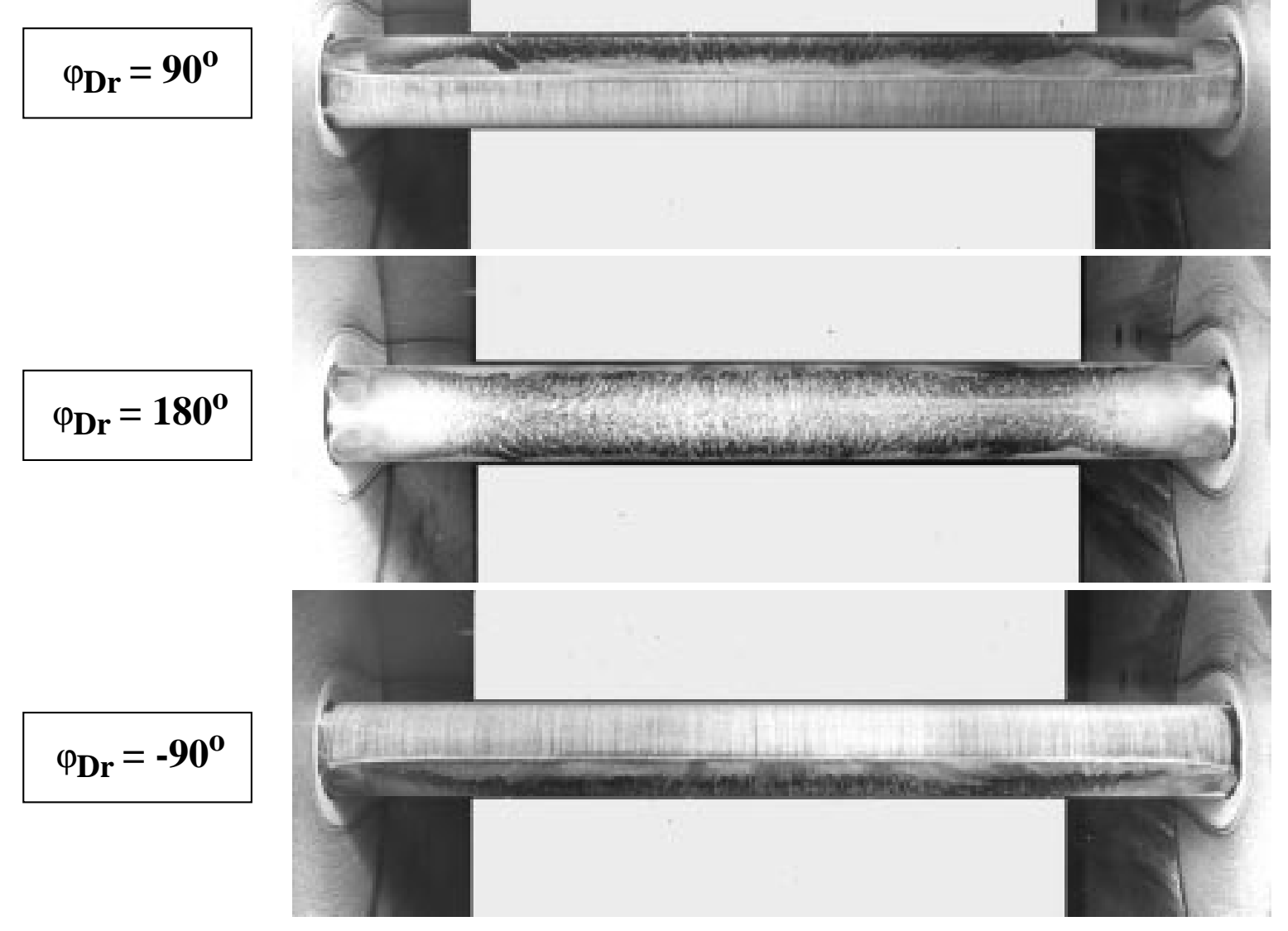

Bild A2.13 Ölanstrichbild bei gemessener Reynoldszahl von $R e_{D}=3,00 \times 10^{6}$ mit $C_{W}=0,90$ 


\section{A.4 Passiv gestörte Zylindergrenzschicht durch Wirbelerzeuger}

\begin{tabular}{|l|c|c|c|c|}
\hline & $\begin{array}{c}\varphi_{T G} \\
/[\mathrm{Grad}]\end{array}$ & $\begin{array}{c}R_{D} \\
/[106]\end{array}$ & $C_{W}$ & $S r$ \\
\hline \hline Bild A3.1 & 55 & 0,20 & 1,20 & 0,22 \\
\hline Bild A3.2 & 55 & 0,40 & 0,48 & $(0,10)$ \\
\hline Bild A3.3 & 55 & 0,53 & 0,51 & 0,25 \\
\hline Bild A3.4 & 55 & 0,94 & 0,64 & 0,30 \\
\hline Bild A3.5 & 55 & 1,61 & 0,71 & 0,32 \\
\hline Bild A3.6 & 55 & 3,06 & 0,90 & 0,34 \\
\hline Bild A4.1 & 80 & 0,32 & 0,45 & $k . A$. \\
\hline Bild A4.2 & 80 & 0,40 & 0,55 & $(0,10)$ \\
\hline Bild A4.3 & 80 & 0,52 & 0,52 & 0,22 \\
\hline Bild A4.4 & 80 & 0,69 & 0,53 & 0,26 \\
\hline
\end{tabular}

Tabelle A3 Übersicht über angefertigte Anstrichbilder bei verschiedenen Reynoldszahlen des laminar-turbulenten Überganges und zwei verschiedenen Azimutpositionen der Wirbelerzeuer. Bei der Anfertigung der Anstrichbilder wurde nur der Bereich um den vorderen Staupunkt eingestrichen. 
Spannweitig-verteilte Turbulenzgeneratoren mit $\Delta y_{T G}=2 D$ bei $\varphi_{T G}=55$ Grad

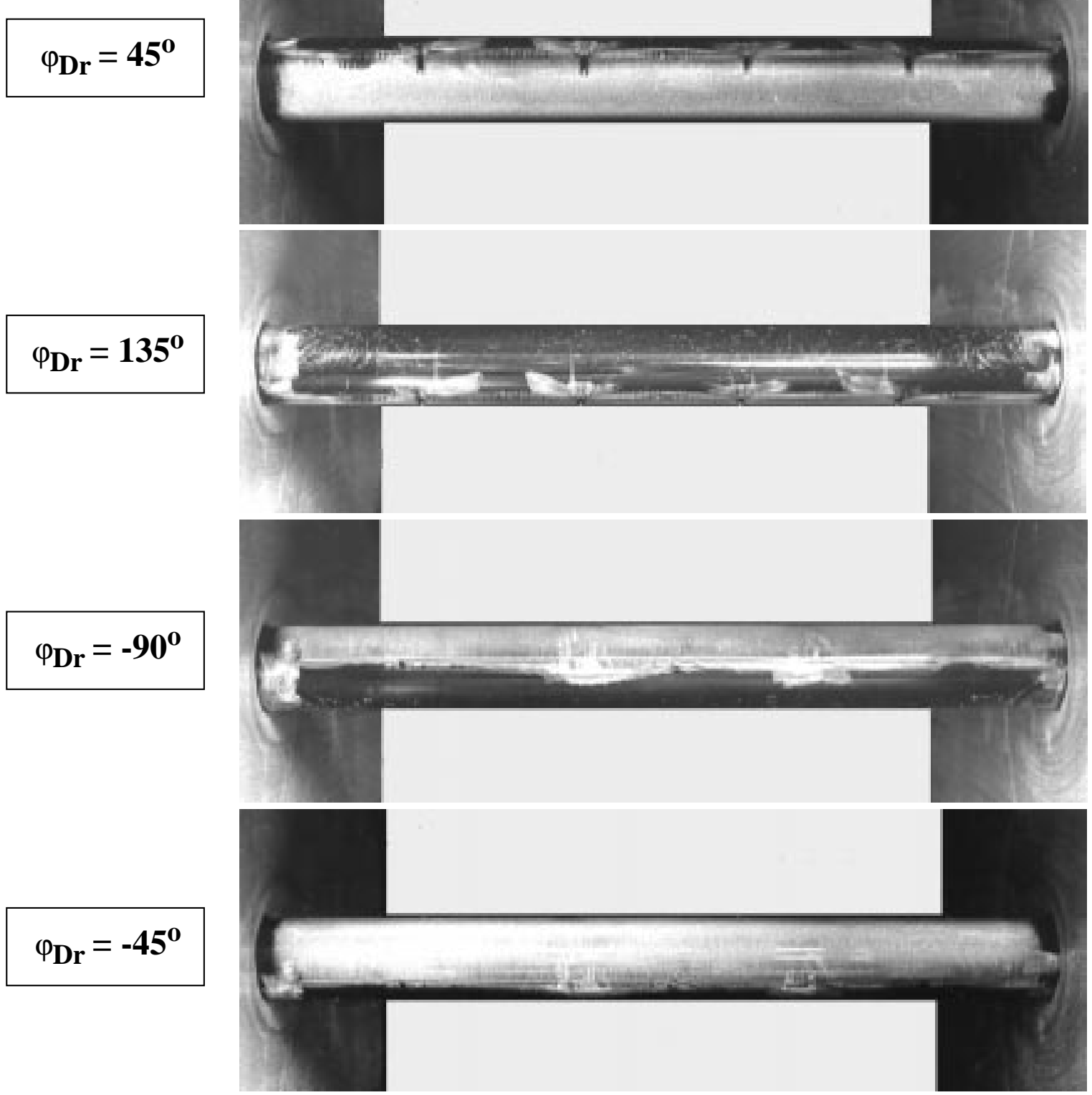

Bild A3.1 Ölanstrichbild bei gemessener Reynoldszahl von $R e_{D}=0,20^{\times} 10^{6}$ mit $C_{W}=1,20$ 


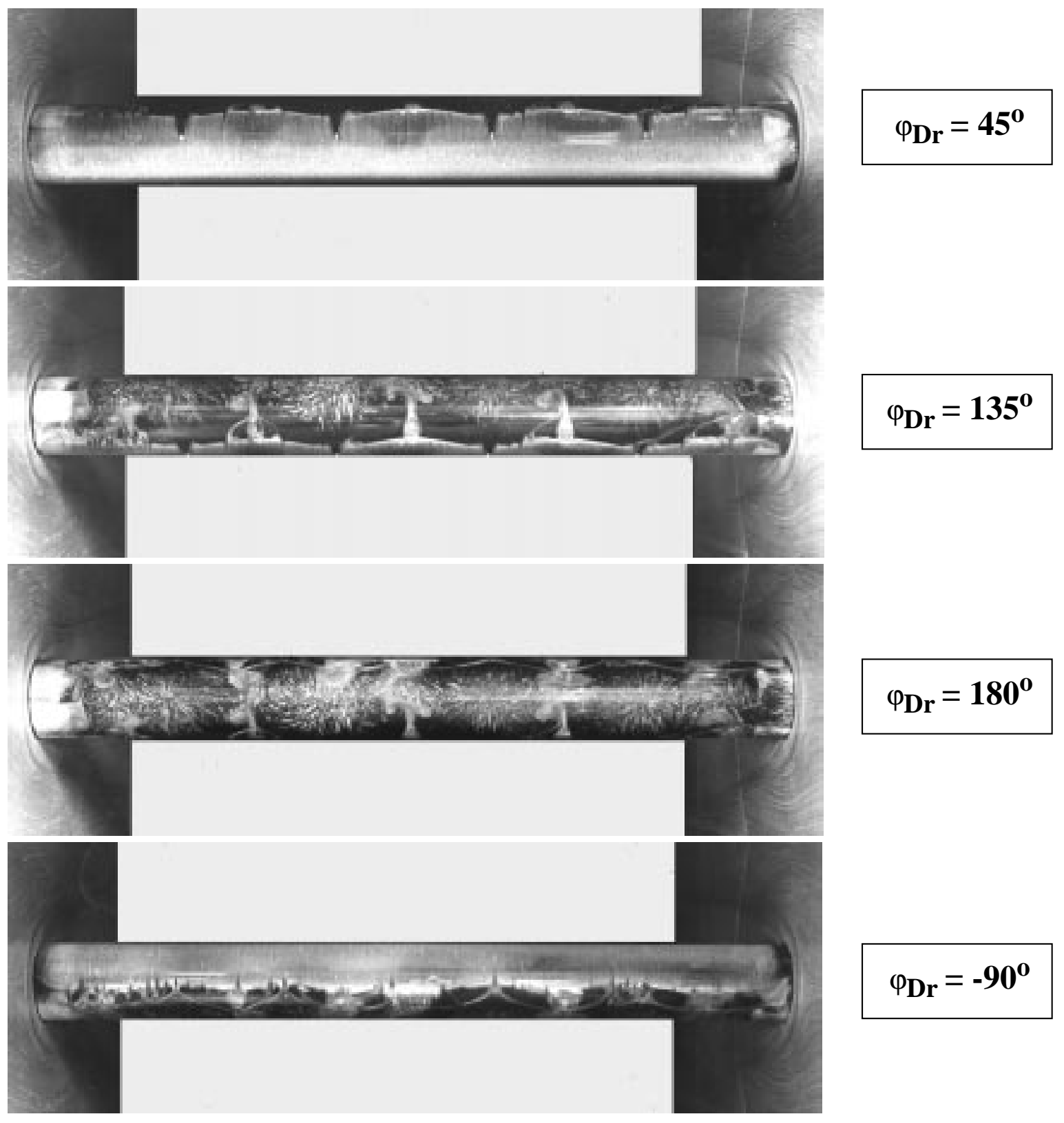

Bild A3.2 Ölanstrichbild bei gemessener Reynoldszahl von $R e_{D}=0,40^{\times 10^{6}}$ mit $C_{W}=0,48$ 


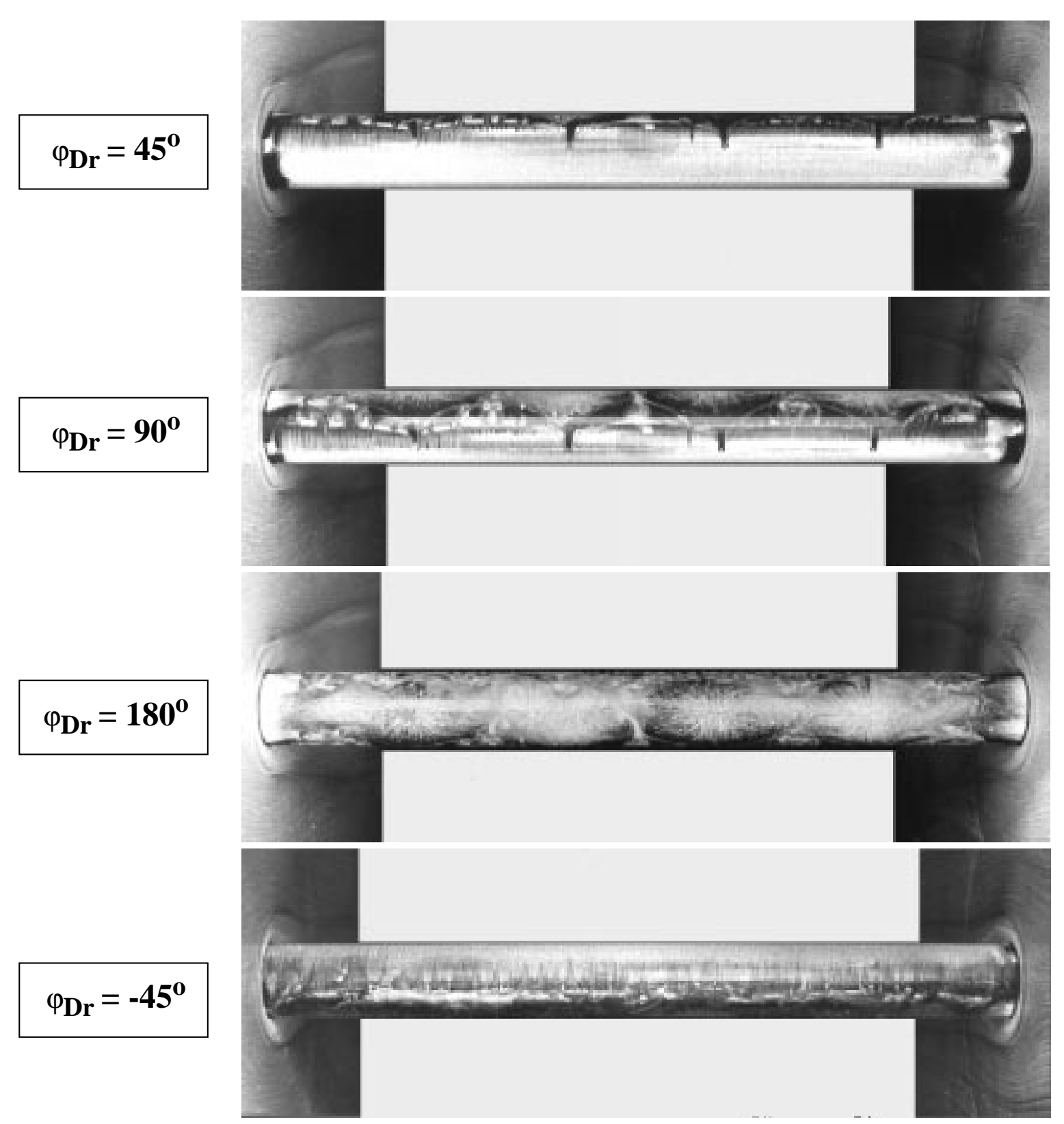

Bild A3.3 Ölanstrichbild bei gemessener Reynoldszahl von $R e_{D}=0,53^{\times} 10^{6}$ mit $C_{W}=0,51$ 


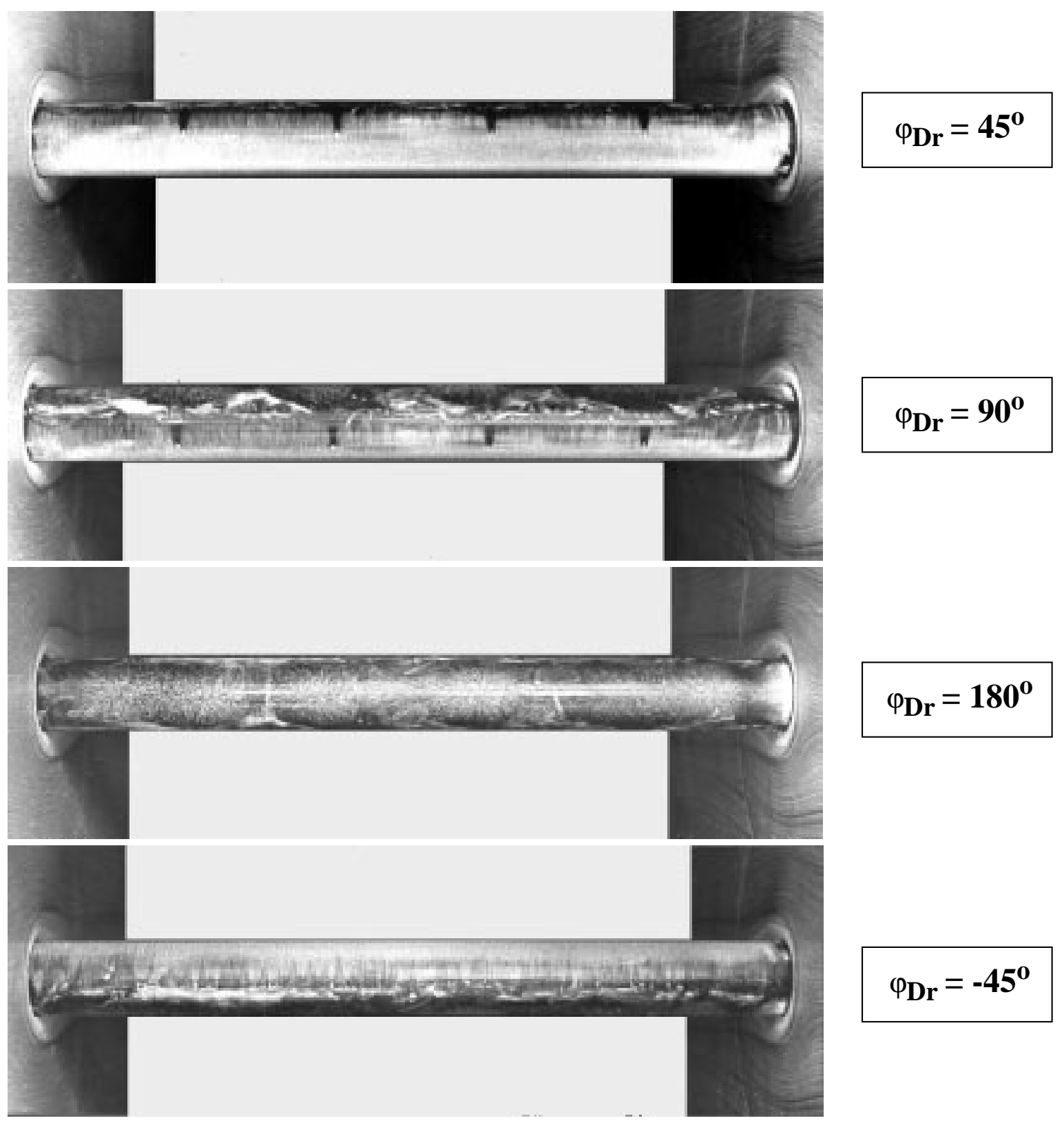

Bild A3.4 Ölanstrichbild bei gemessener Reynoldszahl von $R e_{D}=0,90^{\times 10^{6}}$ mit $C_{W}=0,64$ 


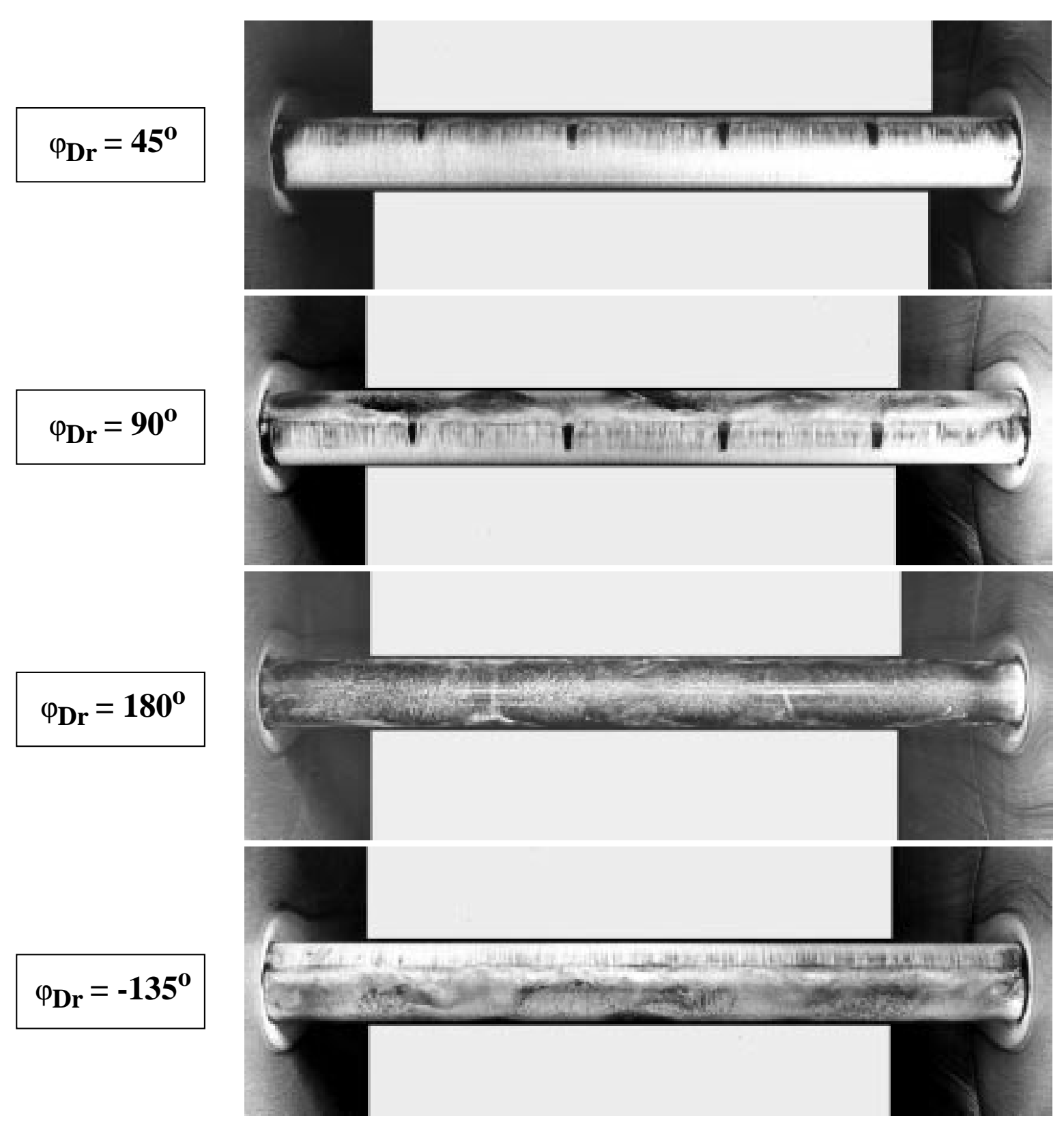

Bild A3.5 Ölanstrichbild bei gemessener Reynoldszahl von $R e_{D}=1,61^{\times} 10^{6}$ mit $C_{W}=0,71$ 


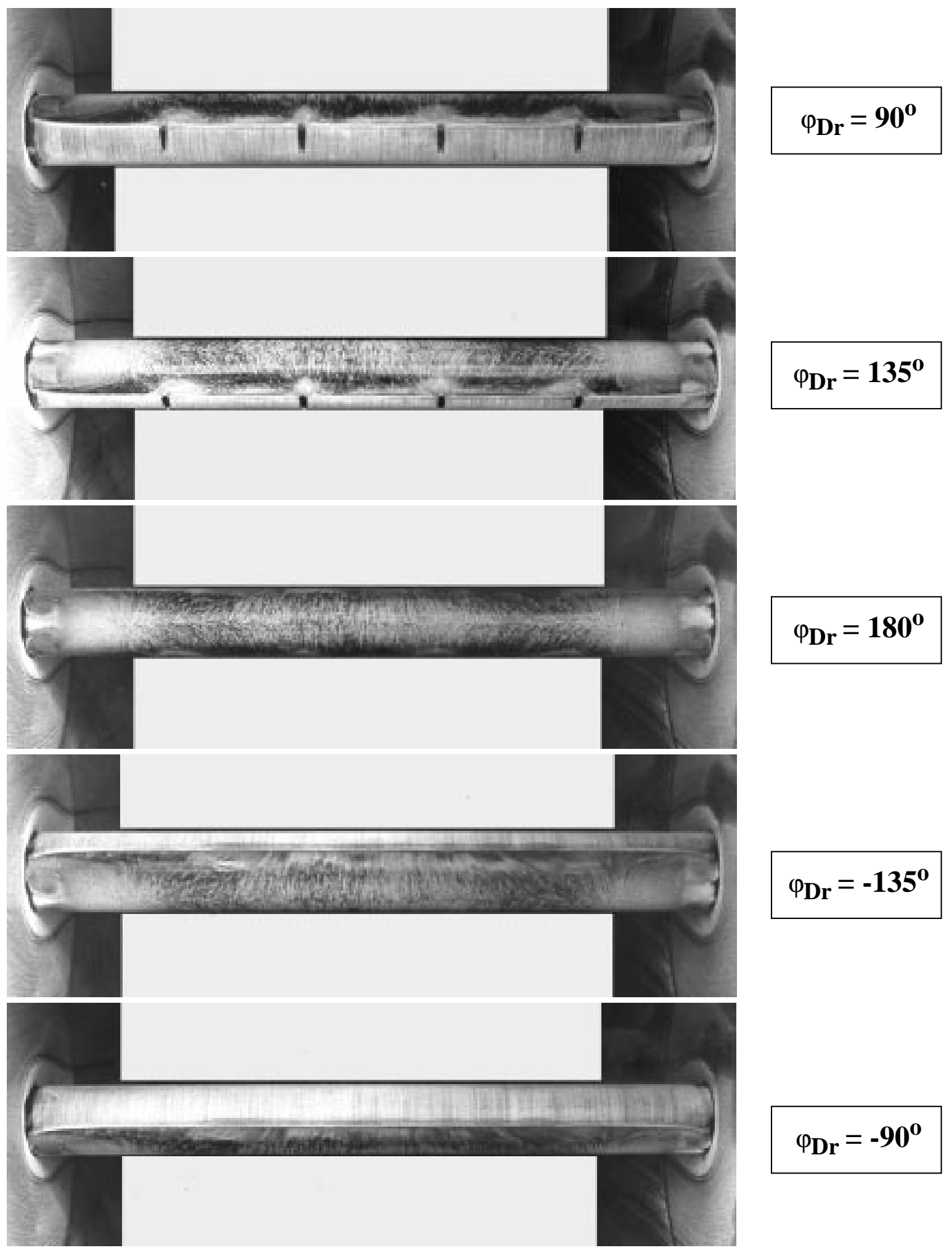

Bild A3.6 Ölanstrichbild bei gemessener Reynoldszahl von $R e_{D}=3,06^{\times} 10^{6}$ mit $C_{W}=0,90$ 
Spannweitig-verteilte Turbulenzgeneratoren mit $\Delta y_{T G}=2 D$ bei $\varphi_{T G}=80 \mathrm{Grad}$
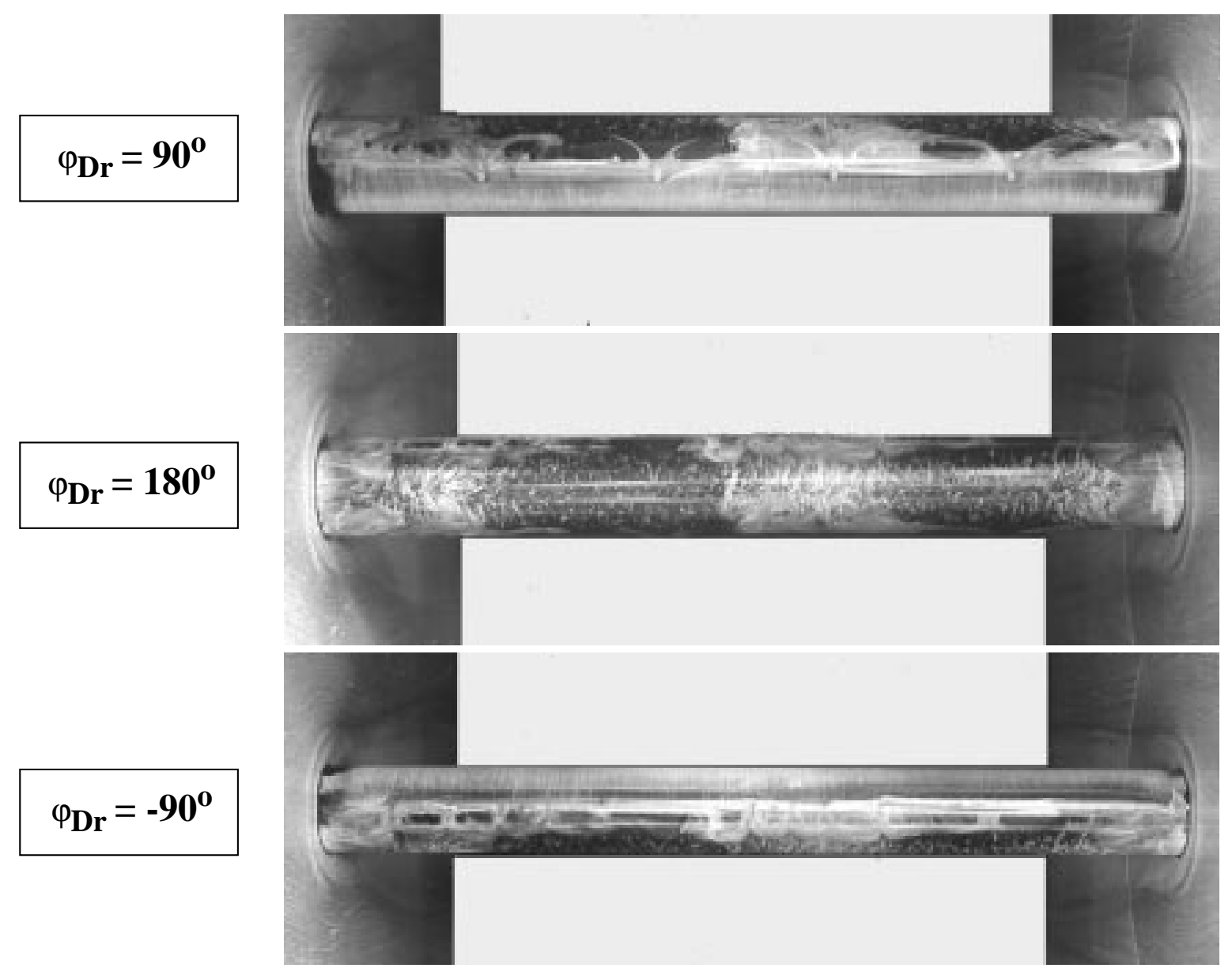

Bild A4.1 Ölanstrichbild bei gemessener Reynoldszahl von $R e_{D}=0,32^{\times} 10^{6}$ mit $C_{W}=0,45$ 


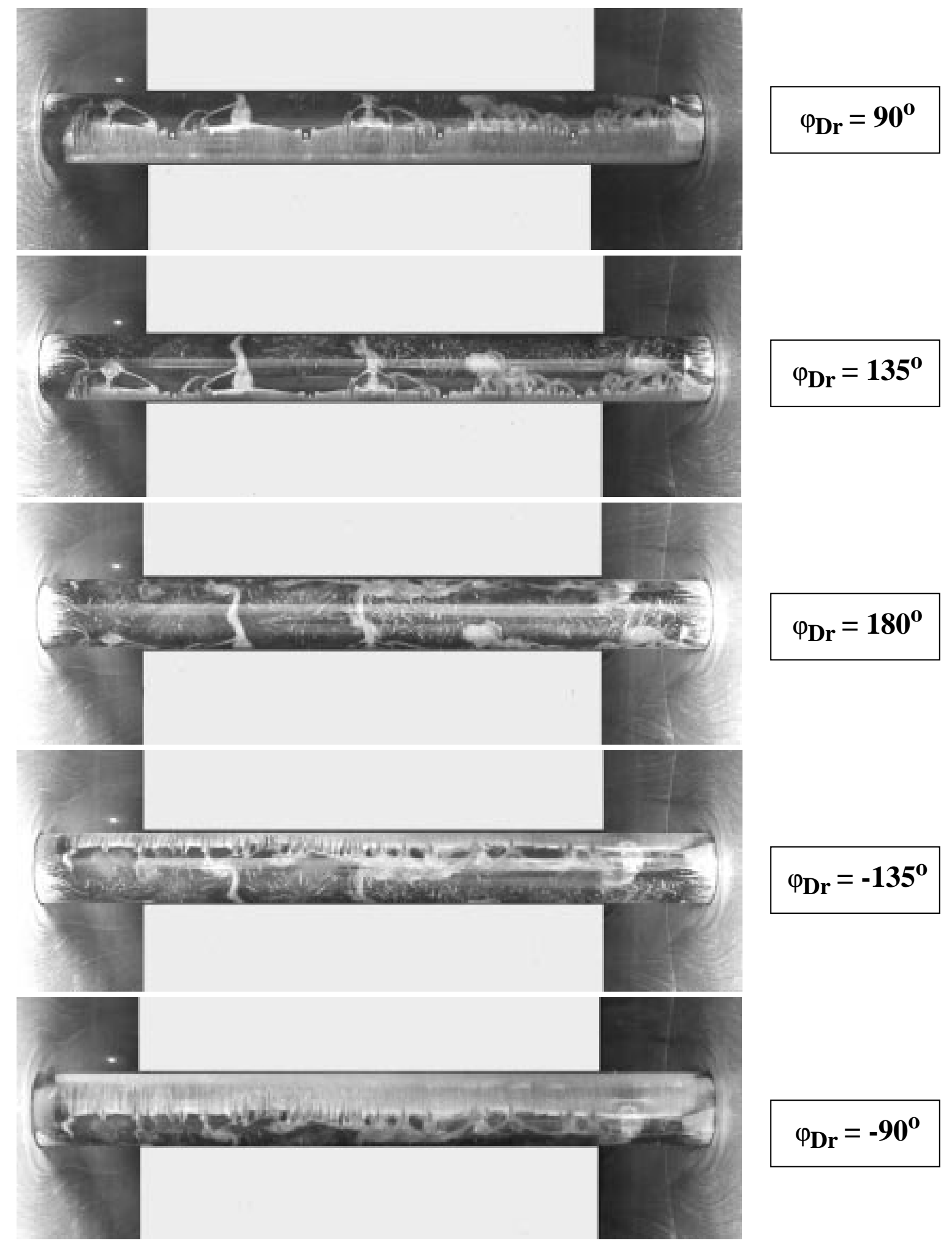

Bild A4.2 Ölanstrichbild bei gemessener Reynoldszahl von $R e_{D}=0,40 \times 10^{6}$ mit $C_{W}=0,55$ 


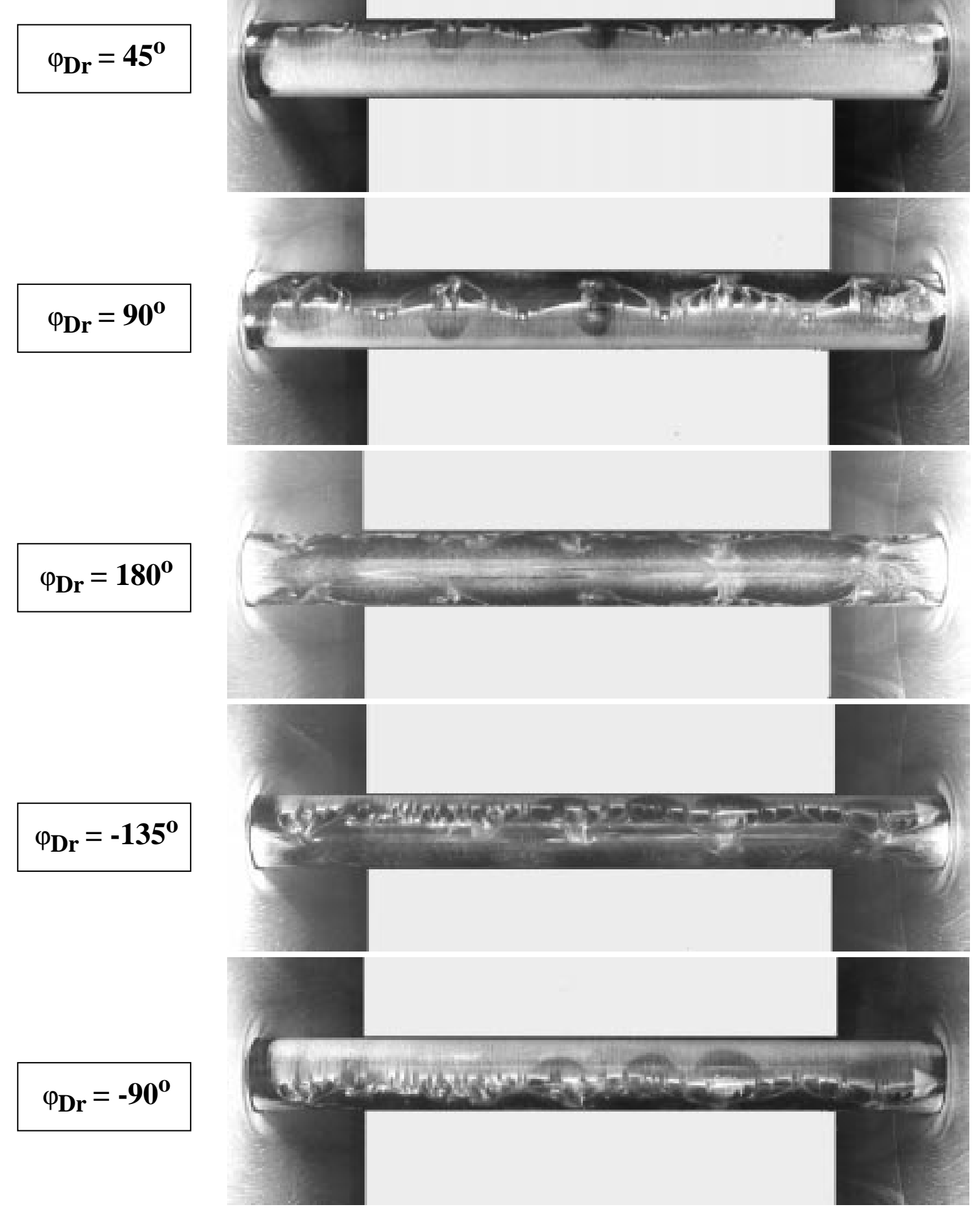

Bild A4.3 Ölanstrichbild bei gemessener Reynoldszahl von $R e_{D}=0,52^{\times} 10^{6}$ mit $C_{W}=0,52$ 


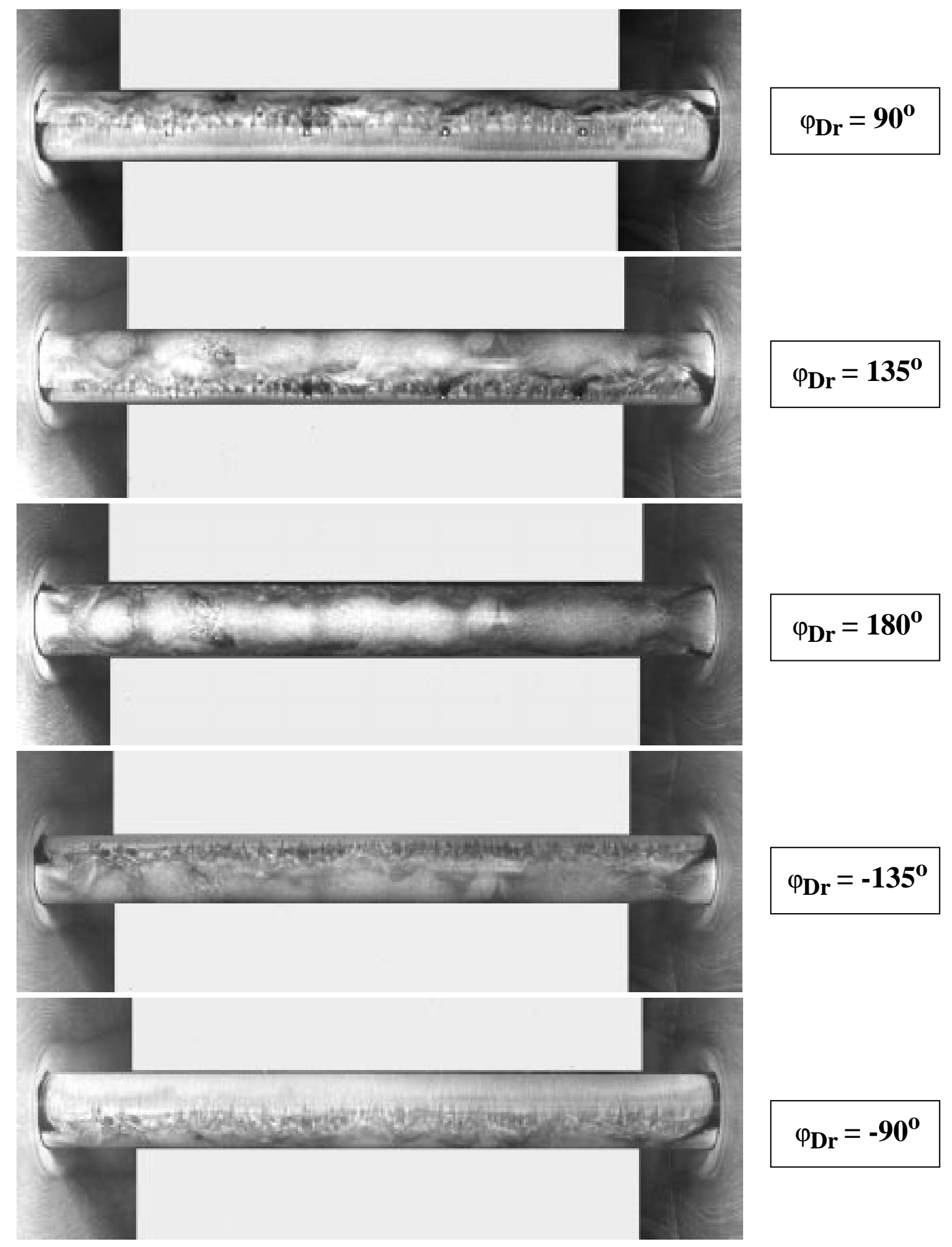

Bild A4.4 Ölanstrichbild bei gemessener Reynoldszahl von $R e_{D}=0,69 \times 10^{6}$ mit $C_{W}=0,58$ 


\section{A.5 Re-gemittelte Widerstandsreduktionen bei aktiver Beeinflussung}

\begin{tabular}{|c|c|c|c|c|c|}
\hline$S r_{E}$ & $\begin{array}{c}y_{E} \\
/[D]\end{array}$ & $\begin{array}{c}\overline{\Delta C_{W, r e l}} \\
/[\%]\end{array}$ & $\begin{array}{c}y_{L} \\
/[D]\end{array}$ & $\begin{array}{c}\text { Spannw. } \\
\text { Symm. }\end{array}$ & Verweis \\
\hline 0,0 & homogen & 18 & - & - & Bild 4.8 \\
\hline 0,0 & $4,4(\max )$ & 24 & $1,7-\underline{\mathbf{3}, \mathbf{3}}-3,3-\underline{\mathbf{1}, 7}$ & asymm. & Bild 4.10 \\
\hline 0,0 & 4,4 (min) & 24 & $3,3-\underline{\mathbf{3}, \mathbf{3}}-3,3$ & symm. & Bild 4.10 \\
\hline 0,0 & $2,2(\max )$ & 25 & $1,7-\underline{\mathbf{2}, \mathbf{5}}-1,7-\underline{\mathbf{2}, \mathbf{5}}-1,7$ & symm. & Bild 4.10 \\
\hline 0,0 & $2,2(\min )$ & 26 & $2,5-\underline{\mathbf{2}, \mathbf{5}}-1,7-\underline{\mathbf{1}, 7}-1,7$ & symm. & Bild 4.10 \\
\hline 0,2 & homogen & 10 & $\underline{\mathbf{2 , 5}}-5,0-\underline{\mathbf{2 , 5}}$ & symm. & Bild 4.13 \\
\hline 0,4 & homogen & 16 & $\underline{\mathbf{1 , 7}}-6,6-\underline{\mathbf{1 , 7}}$ & symm. & Bild 4.13 \\
\hline 0,5 & homogen & 14 & $\underline{\mathbf{1}, 7}-6,6-\underline{\mathbf{1 , 7}}$ & symm. & Bild 4.13 \\
\hline 0,72 & homogen & 25 & $\underline{\mathbf{1 , 7}}-6,6-\underline{\mathbf{1 , 7}}$ & symm. & Bild 4.14 \\
\hline$>1,5$ & homogen & 26 & $\underline{\mathbf{1 , 7}}-6,6-\underline{\mathbf{1 , 7}}$ & symm. & Bild 4.14 \\
\hline 0,2 & $4,4(\max )$ & 21 & $\underline{\mathbf{1 , 7}}-6,6-\underline{\mathbf{1 , 7}}$ & symm. & Bild 4.17 \\
\hline 0.5 & $4,4(\max )$ & 21 & $\underline{\mathbf{1 , 7}}-6,6-\underline{\mathbf{1 , 7}}$ & symm. & Bild 4.17 \\
\hline 0,72 & $4,4(\max )$ & 21 & $\underline{\mathbf{1 , 7}}-6,6-\underline{\mathbf{1 , 7}}$ & symm. & Bild 4.17 \\
\hline$>1,5$ & $4,4(\max )$ & 24 & $\underline{\mathbf{1 , 7}}-\underline{\mathbf{2}, \mathbf{5}}-\underline{\mathbf{1}, 7}-(2,5-\underline{\mathbf{1}, 7})$ & asymm. & Bild 4.17 \\
\hline 0,2 & $2,1(\max )$ & 12 & $\underline{\mathbf{2}, \mathbf{5}}-1,2-\underline{\mathbf{2}, \mathbf{5}}-1,2-\underline{\mathbf{2}, 5}$ & symm. & n.d. \\
\hline 0,5 & $2,1(\max )$ & 13 & $\underline{\mathbf{1}, 7}-1,7-\underline{\mathbf{3}, \mathbf{3}}-1,7-\underline{\mathbf{1 , 7}}$ & symm. & n.d. \\
\hline 0,72 & $2,1(\max )$ & 20 & $\underline{\mathbf{1 , 7}}-1,7-\underline{\mathbf{3}, \mathbf{3}}-1,7-\underline{\mathbf{1 , 7}}$ & symm. & n.d. \\
\hline$>1,5$ & $2,1(\max )$ & 19 & $\underline{\mathbf{1}, 7}-2,5-\underline{\mathbf{1}, 7}-2,5-\underline{\mathbf{1 , 7}}$ & symm. & n.d. \\
\hline$>1,5$ & $8,9(\max )$ & 21 & $2,5-\underline{\mathbf{5 , 0}}-2,5$ & symm. & Bild 4.15 \\
\hline$>1,5$ & $8,9(\min )$ & 21 & $\underline{\mathbf{2 , 5}}-5,0-\underline{\mathbf{2 , 5}}$ & symm. & Bild 4.15 \\
\hline$>1,5$ & $4,4(\max )$ & 24 & $\underline{\mathbf{1 , 7}}-\underline{\mathbf{2}, 5}-\underline{\mathbf{1}, 7}-(2,5-\underline{\mathbf{1}, 7})$ & asymm. & Bild 4.15 \\
\hline$>1,5$ & $4,4(\min )$ & 25 & $2,5-\underline{\mathbf{5 , 0}}-2,5$ & symm. & Bild 4.15 \\
\hline$>1,5$ & $2,9(\max )$ & 18 & $\underline{\mathbf{2}, 5}-5,0-\underline{\mathbf{2}, 5}$ & symm. & Bild 4.16 \\
\hline$>1,5$ & $2,9(\min )$ & 26 & $\underline{\mathbf{3}, \mathbf{3}}-3,3-\underline{\mathbf{3}, \mathbf{3}}$ & symm. & Bild 4.16 \\
\hline
\end{tabular}




\begin{tabular}{|c|c|c|c|c|c|}
\hline$S r_{E}$ & $\begin{array}{c}y_{E} \\
/[D]\end{array}$ & $\begin{array}{c}\overline{\Delta C_{W, r e l}} \\
/[\%]\end{array}$ & $\begin{array}{c}y_{L} \\
/[D]\end{array}$ & $\begin{array}{c}\text { Spannw. } \\
\text { Symm. }\end{array}$ & Verweis \\
\hline$>1,5$ & $2,1(\max )$ & 19 & $\underline{\mathbf{2}, \mathbf{5}}-1,7-\underline{\mathbf{1}, 7}-1,7-\underline{\mathbf{2}, 5}$ & symm. & Bild 4.16 \\
\hline$>1,5$ & $2,1(\max )$ & 22 & $\underline{\mathbf{3}, \mathbf{3}}-3,3-\underline{\mathbf{3}, \mathbf{3}}$ & symm. & Bild 4.16 \\
\hline
\end{tabular}

Tabelle A4 Übersicht über spannweitig homogene oder periodische Beeinflussung durch Absaugen mit $Q_{E}=1,92 \mathrm{~m}^{3} / \mathrm{h}$ und akustischer Anregung mit $L_{E}=92 \mathrm{~dB}$ bei Variation der Anregefrequenz $S r_{E}$ mit Angabe der mittleren Widerstandsreduktion $\Delta \mathrm{C}_{\mathrm{W} \text {,rel }}$

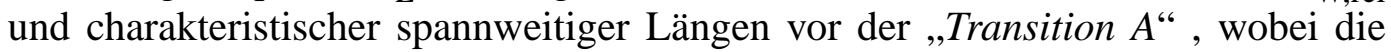
spannweitigen Bereiche mit minimalem Druckbeiwert fett unterstrichen sind. Weiterhin ist der spannweitige Symmetriecharakter der „Transition A“ angegeben. 


\section{A.6 Re-Bereiche beim laminar-turbulenten Übergang der Zylindergrenzschicht}

\begin{tabular}{|c|c|c|c|c|c|c|c|}
\hline $\begin{array}{c}R e- \\
\text { Bereich }\end{array}$ & $\begin{array}{c}R e_{D} \\
/\left[10^{6}\right]\end{array}$ & $S r$ & $\begin{array}{l}\text { azimut. } \\
\text { Ablö- } \\
\text { sung }\end{array}$ & $\begin{array}{c}y_{L} \\
/[D]\end{array}$ & $\begin{array}{l}\text { Ablöse- } \\
\text { Struktur }\end{array}$ & $\begin{array}{l}\text { Wirbel } \\
\text { struk- } \\
\text { tur }\end{array}$ & Verhalten \\
\hline $\begin{array}{l}\text { unter- } \\
\text { kritisch }\end{array}$ & $\begin{array}{l}0,05 \\
\text { bis } \\
0,15\end{array}$ & 0,20 & symm. & - & $\begin{array}{l}\text { beidseitig; } \\
\text { homogen } \\
1 \text { Linie }\end{array}$ & $\begin{array}{l}\text { Quer- } \\
\text { wirbel }\end{array}$ & stabil \\
\hline $\begin{array}{l}\text { unter- } \\
\text { kritisch }\end{array}$ & $\begin{array}{l}0,15 \\
\text { bis } \\
0,20\end{array}$ & 0,20 & asymm. & $\begin{array}{l}2 ; \\
(3,3 ; 2,5 ; \\
2,0 ; 1,7 ; \\
1,2)^{*}\end{array}$ & $\begin{array}{l}\text { homogen; } \\
1 \text { Linie/ } \\
2 \text { Linien }\end{array}$ & $\begin{array}{l}\text { Quer- } \\
\text { wirbel }\end{array}$ & $\begin{array}{l}\text { instabil, } \\
\text { Grenzschicht- } \\
\text { Scherschicht- } \\
\text { Wechselwirkung }\end{array}$ \\
\hline kritisch & $\begin{array}{l}0,20 \\
\text { bis } \\
0,30\end{array}$ & 0,20 & symm. & - & $\begin{array}{l}\text { homogen; } \\
2 \text { Linien }\end{array}$ & $\begin{array}{l}\text { Quer- } \\
\text { wirbel }\end{array}$ & stabil \\
\hline kritisch & $\begin{array}{l}0,30 \\
\text { bis } \\
0,32\end{array}$ & $\begin{array}{l}0,20 \\
\text { bis } \\
0,16\end{array}$ & symm. & 0,14 & $\begin{array}{l}\text { period. } \\
\text { und } \\
2 \text { Linien }\end{array}$ & $\begin{array}{l}\text { Quer- } \\
\text { und } \\
\text { Längs- } \\
\text { wirbel }\end{array}$ & instabil \\
\hline kritisch & $\begin{array}{l}0,32 \\
\text { bis } \\
0,33\end{array}$ & 0,33 & asymm. & - & $\begin{array}{l}\text { einseitig } \\
\text { transitio- } \\
\text { nelle } \\
\text { Blase, } \\
\text { homogen }\end{array}$ & $\begin{array}{l}\text { Quer- } \\
\text { wirbel }\end{array}$ & stabil \\
\hline kritisch & $\begin{array}{l}0,33 \\
\text { bis } \\
0,35\end{array}$ & $\begin{array}{l}0,33 \\
\text { abf. bis } \\
0,30\end{array}$ & asymm. & $\begin{array}{l}6 ; \\
3\end{array}$ & $\begin{array}{l}\text { einseitig } \\
\text { transitio- } \\
\text { nelle } \\
\text { Blase, } \\
\text { periodisch }\end{array}$ & $\begin{array}{l}\text { Quer- } \\
\text { und } \\
\text { Längs- } \\
\text { wirbel }\end{array}$ & $\begin{array}{l}\text { instabil, } \\
\text { Inst. d. einseiti- } \\
\text { gen Ablöseblase }\end{array}$ \\
\hline $\begin{array}{l}\text { über- } \\
\text { kritisch }\end{array}$ & $\begin{array}{l}0,35 \\
\text { bis } \\
1,00\end{array}$ & 0,50 & symm. & - & $\begin{array}{l}\text { beidseitig; } \\
\text { trans. } \\
\text { Blase, } \\
\text { homogen }\end{array}$ & $\begin{array}{l}\text { Quer- } \\
\text { wirbel }\end{array}$ & stabil \\
\hline $\begin{array}{l}\text { oberer } \\
\text { Über- } \\
\text { gang }\end{array}$ & $\begin{array}{l}1,00 \\
\text { bis } \\
2,20\end{array}$ & 0,10 & symm. & $\begin{array}{l}3,3 ; 2,52 \\
0 ; 1,7 ; 1,2\end{array}$ & $\begin{array}{l}\text { beidseitig, } \\
\text { periodisch }\end{array}$ & $\begin{array}{l}\text { Längs- } \\
\text { wirbel }\end{array}$ & $\begin{array}{l}\text { instabil, } \\
\text { Inst. der Ablöse- } \\
\text { blasen }\end{array}$ \\
\hline
\end{tabular}




\begin{tabular}{|l|l|l|l|l|l|l|l|}
\hline $\begin{array}{l}R e- \\
\text { Bereich }\end{array}$ & $\begin{array}{c}R e_{D} \\
/\left[10^{6}\right]\end{array}$ & \multicolumn{1}{|c|}{$S r$} & $\begin{array}{c}\text { azimut. } \\
\text { Ablö- } \\
\text { sung }\end{array}$ & $\begin{array}{c}y_{L} \\
/[D]\end{array}$ & $\begin{array}{c}\text { Ablöse- } \\
\text { Struktur }\end{array}$ & $\begin{array}{c}\text { Wirbel } \\
\text { struk- } \\
\text { tur }\end{array}$ & Verhalten \\
\hline \hline $\begin{array}{l}\text { oberer } \\
\text { Über- } \\
\text { gang }\end{array}$ & $\begin{array}{l}2,20 \\
\text { bis } \\
2,50\end{array}$ & 0,10 & symm. & $?$ & beidseitig & $\begin{array}{l}\text { Längs- } \\
\text { wirbel }\end{array}$ & $\begin{array}{l}\text { instabil; } \\
\text { Umordnung der } \\
\text { Längswirbel zu } \\
\text { Querwirbeln }\end{array}$ \\
\hline $\begin{array}{l}\text { trans- } \\
\text { krit. } \\
\text { Bereich }\end{array}$ & $\begin{array}{l}2,50 \\
\text { bis } \\
3,50\end{array}$ & 0,20 & symm. & $6 ; 2$ & $\begin{array}{l}\text { beidseitig; } \\
\text { Linien, } \\
\text { homogen }\end{array}$ & $\begin{array}{l}\text { Quer- } \\
\text { wirbel }\end{array}$ & stabil/instabil \\
\hline $\begin{array}{l}\text { trans- } \\
\text { kritisch }\end{array}$ & $\begin{array}{l}\text { ab } \\
3,50\end{array}$ & $\begin{array}{l}0,20 \\
\text { stei- } \\
\text { gend }\end{array}$ & symm. & - & $\begin{array}{l}\text { beidseitig; } \\
\text { 1 Linie, } \\
\text { homogen }\end{array}$ & $\begin{array}{l}\text { Quer- } \\
\text { wirbel }\end{array}$ & stabil \\
\hline
\end{tabular}

Tabelle A5 Übersicht über charakteristische räumliche und zeitliche Skalen der abgelösten Zylinderströmung bei den Übergängen vom unterkritischen bis transkritischen Reynoldszahlbereich mit Angabe der dominierenden Wirbelstruktur und des Stabilitätszustandes 


\section{Danksagung}

Die vorliegende Forschungsarbeit entstand während meiner Tätigkeit als Doktorand am Institut für Strömungsmechanik des Deutschen Zentrums für Luft- und Raumfahrt e.V. (DLR) in Göttingen. Dem Institutsleiter Prof. Dr. G.E.A. Meier danke ich für sein großes Interesse an dieser Arbeit und für die Möglichkeit, über die wissenschaftliche Tätigkeit hinaus, sich engagieren zu können.

An dieser Stelle möchte ich mich bei Prof. Dr. H. Eckelmann für die Übernahme des Hauptreferats bedanken. Seine bereitwillige Unterstützung in Form von lebendigen Diskussionen insperierten mich insbesondere beim Abschluss der Dissertation noch neue Gedanken aufzugreifen.

Mein besonderer Dank gilt Herrn Priv. Doz. Dr. U. Ch. Dallmann für die Anregung des Themas, für die Übernahme des Korreferats sowie für die wissenschaftliche Betreuung. Durch zahlreiche Diskussionen und kritische Anmerkungen hat er wesentlich zum Gelingen dieser Arbeit beigetragen. Ebenso möchte ich Herrn Dr. G. Schewe danken. Durch seine Unterstützung konnte ein großer Umfang an experimentellen Untersuchungen am Hochdruckwindkanal Göttingen (HDG) durchgeführt werden.

Weiterhin möchte ich insbesondere einer Reihe von Kollegen der Abteilung Transition und Turbulenz - Dr. H.-P. Kreplin, Dr. E. Janke, Dipl.-Ing. S. Hein, Prof. Dr. V. Theofilis und Dr. W. Koch - für die interessanten und inspirierenden Gespräche danken.

Dank möchte ich auch den Mitarbeitern Herrn D. Baumgarten, Herrn H. Denecke, Herrn H. Mattner sowie Herrn D. Hübner für die tatkräftige Unterstützung bei der Vorbereitung und Durchführung der Experimente am 1-Meter-Kanal danken. Dem Team der Abteilung Windkanäle beim Deutsch-Niederländischen-Windkanalverbund (DNW) möchte ich ebenfalls danken. Durch den Einsatz ihrer Datenerfassung- und Steuerungstechnik stand mir am HDG ein hochwertiges System zur Verfügung, dass erst die Durchführung der zum Teil komplexen Messvorhaben ermöglichte. Keinesfalls selbstverständlich schätze ich das eingebrachte technische Wissen und Können sowie den persönlichen Einsatz von Herrn H.-J. Bendig für das Gelingen der dort durchgeführten Experimente. Gleichfalls danke ich den Kollegen der Fertigung und Konstruktion für die Herstellung der verschiedenen Zylindermodelle und für die Anpassung der Messstrecken des HDG an die experimentell technischen Bedürfnisse. Weiterhin danke ich den Elektronikern der Werkstatt und des Institutes für Aeroelastik, mit deren Hilfe die sichere Anwendung der dynamischen Druckaufnehmer und der Aktuatorik gewährleistet wurde.

Unentbehrliche Hilfe liegt auch im Detail. Für den ein oder anderen Tip bei der Erstellung von Grafiken mit Matlab-Skripten sei Dr. G. Dietz, für die Bearbeitung des Textes unter FrameMaker Dr. M. Klein gedankt. Für die stets freundliche Unterstützung bei der Suche nach Literaturquellen möchte ich Frau A. Taschner und Frau B. Bode danken.

Meinen herzlichsten Dank möchte ich meiner Mutter, meinem Vater, meinem Daddy und meinen Schwestern sowie nahestehenden Freunden aussprechen. Durch ihren Beistand und Rückhalt konnte ich diese wissenschaftliche Arbeit beenden. 